University of Louisville

ThinkIR: The University of Louisville's Institutional Repository

$12-2016$

\title{
Transient receptor potential cation channel, subfamilies V, member 1 (TRPV1) and M, member 1 (TRPM1) contribute to neural signaling in mouse retina.
}

Jennifer Noel

University of Louisville

Follow this and additional works at: https://ir.library.louisville.edu/etd

Part of the Biology Commons, and the Molecular and Cellular Neuroscience Commons

\section{Recommended Citation}

Noel, Jennifer, "Transient receptor potential cation channel, subfamilies V, member 1 (TRPV1) and M, member 1 (TRPM1) contribute to neural signaling in mouse retina." (2016). Electronic Theses and Dissertations. Paper 2572.

https://doi.org/10.18297/etd/2572

This Doctoral Dissertation is brought to you for free and open access by ThinkIR: The University of Louisville's Institutional Repository. It has been accepted for inclusion in Electronic Theses and Dissertations by an authorized administrator of ThinkIR: The University of Louisville's Institutional Repository. This title appears here courtesy of the author, who has retained all other copyrights. For more information, please contact thinkir@louisville.edu. 


\title{
TRANSIENT RECEPTOR POTENTIAL CATION CHANNEL, SUBFAMILIES V, MEMBER 1 (TRPV1) AND M, MEMBER 1 (TRPM1) CONTRIBUTE TO NEURAL SIGNALING IN MOUSE RETINA
}

\author{
By \\ Jennifer Noel \\ B.S., University of Louisville, 2007

\begin{abstract}
A Dissertation
Submitted to the Faculty of the

School of Medicine of the University of Louisville

in Partial Fulfillment of the Requirements for the Degree of
\end{abstract}

Doctor of Philosophy in Anatomical Sciences and Neurobiology

Department of Anatomical Sciences and Neurobiology

University of Louisville

Louisville, Kentucky

December 2016 



\section{TRANSIENT RECEPTOR POTENTIAL CATION CHANNEL, SUBFAMILIES V, MEMBER 1 (TRPV1) AND M, MEMBER 1 (TRPM1) CONTRIBUTE TO NEURAL SIGNALING IN MOUSE RETINA}

\section{By}

Jennifer Noel

B.S., University of Louisville, 2007

A Dissertation Approved on

July 8, 2016

by the following Dissertation Committee:

Maureen McCall, Ph.D.

Martha Bickford, Ph.D.

Paul DeMarco, Ph.D.

Ronald Gregg, Ph.D.

Robin Krimm, Ph.D.

Nicholas Mellen, Ph.D. 


\section{ACKNOWLEDGMENTS}

As a single mom of young children, taking on my dream of earning a Doctor of Philosophy degree to pursue research was a task I obviously will not accomplish alone. Although my name appears of the front page of this dissertation, many people have contributed to its production and deserve equal credit. First, I owe my gratitude to my parents, Mike and Hilda Noel, my children, Sophie and Owen, my partner, Joseph Walker, and my grandmother, Joan Noel, whose love, help, support, and patience made this possible. Your sacrifices have helped me accomplish my dream and I am eternally grateful. I also would like to express my deep appreciation and gratitude to my advisor, Dr. Maureen McCall, for the guidance and mentorship she provided to me. She was my original mentor for my Undergraduate thesis and is the reason I continued in research. I would like to thank my committee members, Drs. Ronald Gregg, Martha Bickford, Paul DeMarco, Nicholas Mellen and Robin Krimm for the guidance, thoughtprovoking suggestions, and endless patience as I struggled through the completion of this manuscript. Dr. Gregg, in many ways, has served as a second mentor to me and a source of incredible knowledge. Paul was my mentor when I first pursued a Master's degree and has been a vital source of encouragement. I also owe my gratitude to Dr. Henry Kaplan for giving me my first full-time job in research. He provided incredible opportunities to work in a variety of capacities 
on innovative experiments. Dr. William Guido, Chair of our department and teacher and advocate for students, gave his time to teach me experimental techniques and provide any resources I needed. Dr. Bart Borghuis, a collaborator in the experiments within this dissertation, provided vital help, support and guidance, which has expanded my understanding of neuroscience in many ways. I also am indebted to all the McCall and Gregg, Kaplan and Borghuis lab members, who are too numerous to mention, for technical, logical, emotional, and inspirational support. It has not only been a pleasure working with you all, but you are my lifetime friends. Finally, I appreciate the financial support from the NEI, Sigma Xi, and Research to Prevent Blindness that funded the research discussed in this dissertation. 


\begin{abstract}
TRANSIENT RECEPTOR POTENTIAL CATION CHANNEL, SUBFAMILIES V, MEMBER 1 (TRPV1) AND M, MEMBER 1 (TRPM1) CONTRIBUTE TO NEURAL SIGNALING IN MOUSE RETINA
\end{abstract}

Jennifer Noel

July 07,2016

The retina processes light information through parallel pathways in order to extract and encode the visual scene. Light information is transmitted to the brain through approximately 30 ganglion cells (GCs), the retinal output neurons. Trp channels modulate the responses of retinal neurons within specific pathways. The study of the expression and function of the majority of Trp channels in the retina is largely in its infancy. My dissertation first investigated the expression and function of the transient receptor potential vanilloid-1 (TRPV1) receptor/channel in the retina. TRPV1, the first cloned and most highly studied Trp channel in the peripheral nervous system, is a non-selective cation channel with an affinity for $\mathrm{Ca}^{2+}$. The channel can be activated by capsaicin, acid, endovanilloids, noxious heat or pressure (Moreira et al., 2012). Located on the peripheral and central terminals of nociceptive fibers in the PNS and in limited areas of the CNS (Cavanaugh et al, 2011b). TRPV1 plays a role in inflammation, chronic pain, nociceptor sensitization and desensitization, long-term depression 
and potentiation, and apoptosis. The role of TRPV1 in the retina is not known. Using the electroretinogram (ERG), a mass potential that assesses the function of photoreceptors and bipolar cells, the TRPV1 knockout mouse appears normal. However, TRPV1 is thought to play a role in calcium regulation and glaucoma (Sappington et al., 2009 \& Leonelli et al., 2010) so we investigated its role in normal visual transduction in the inner retina. To investigate TRPV1 modulation, I recorded GC spiking responses to light stimuli from mice which either express or lack TRPV1 protein. I found that TRPV1 is critical for:

1. GC responses to dim light.

2. Sustained responses to light

3. Surround suppression of GCs to large spots.

Further, I investigate the specific retinal cells that express TRPV1. I used TRPV $1^{\text {cre }}$ mice with genetic or viral methods to fluorescently label neurons that express TRPV1. I determined TRPV1 is expressed in four classes of amacrine and three classes of ganglion cells in the inner retina. My results indicate TRPV1 activity in the amacrine cells enhances the sustained spiking responses in GCs. In this way, TRPV1 likely enhances the perception of subtle details in the visual world. TRPV1 also is expressed in subsets of intrinsically photosensitive GCs, which are known to play a role in circadian photoentrainment. TRPV1 therefore has the potential to modulate circadian photoentrainment or other non-image forming visual functions as well.

The role of TRPM1 in the retina is well known. It is required for signaling through the ON pathway, which detects light increments. Responses through the 
ON pathway are initiated by synapses between rod and cone photoreceptors with ON bipolar cells (BCs). The human disease, complete congenital stationary night blindness (cCSNB) results from a disruption in signaling within the ON BC mGluR6 G-protein coupled cascade, which culminates in the opening of the TRPM1 channel and signaling through ON BCs. I helped expand our understanding of the role of TRPM1 in the retina by investigating the expression and function of leucine rich repeat immunoglobulin like transmembrane protein 3 (LRIT3), a novel protein component in the mGluR6-TRPM1 signalplex that was found mutated within cCSNB patients and a knockout mouse (Zeitz et al., 2013; Neuillé et al., 2014). The function of LRIT3 within the cascade remains unknown. To better understand the role of LRIT3, we examined retinal structure and function. We compared the structure of the pre and postsynaptic elements in the OPL of WT and $\mathrm{Lrit3}^{-/}$mice using a variety of antibodies and with confocal microscopy. We assessed overall retinal function with ERG and GC spontaneous and visually evoked activity with single cell and multielectrode array recordings. The overall laminar structure of the Lrit3\% retina is similar to WT. Consistent with published results and other cCSNB mouse models, Lrit3\% mouse dark- and lightadapted ERGs have a normal a-wave, but lack a b-wave. The dendritic terminals of Lrit3 $^{--}$ON BCs lack expression of nyctalopin and TRPM1. Lrit3\% mice significantly differ from other cCSNB mutants. Cone ON BCs lack expression of mGluR6, GPR179 and RGS11, whereas rod BCs maintain expression of these proteins. LRIT3 is necessary for expression and localization of nyctalopin and TRPM1 to the ON BC dendrites. As expected there are no ON responses, but 
surprisingly very few ( 22\%) Lrit3\% GCs have even OFF responses. Lrit3\% OFF BCs express functional kainate glutamate receptors. However, Lrit3\% OFF BC and OFF GCs have significantly smaller response to light decrements than WT. Like all other mouse models of CCSNB, LRIT3 is critical to signaling in ON BCs, however, unlike all other cCSNB models, LRIT3 also has a trans-synaptic role in enhancing glutamate transmission from cones to BCs. 


\section{TABLE OF CONTENTS}

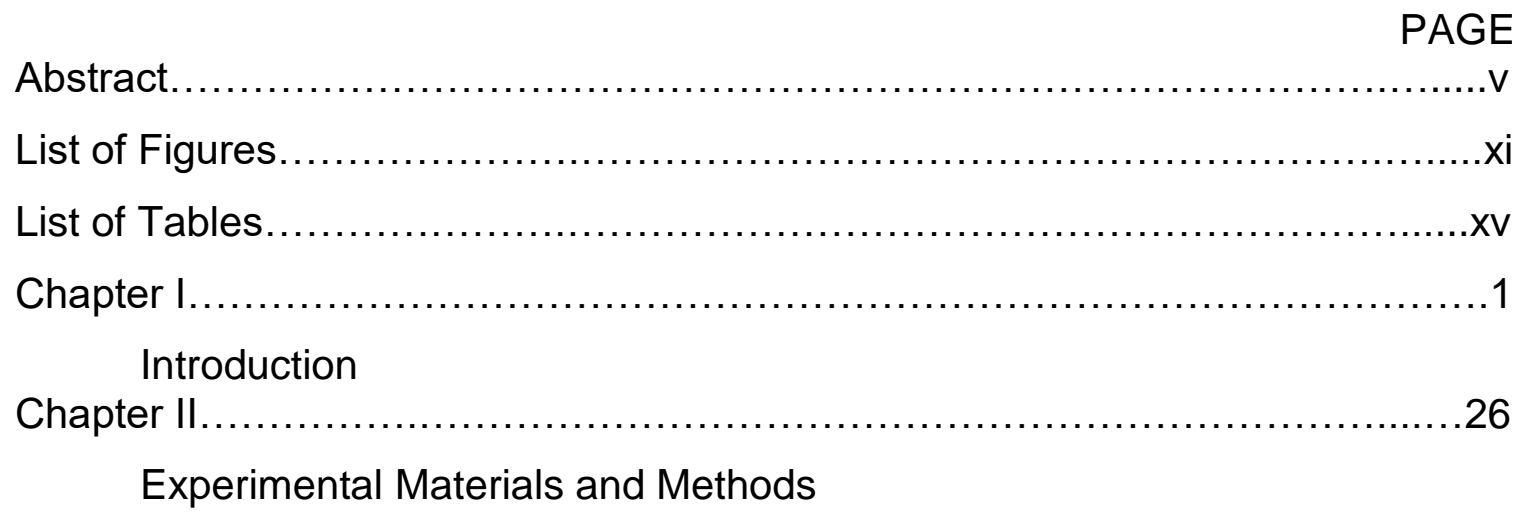

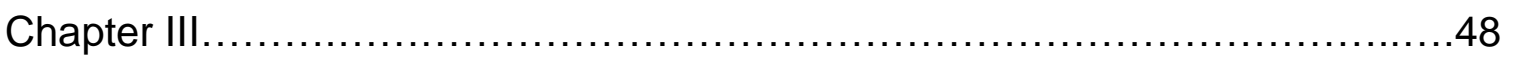

TRPV1 Modulates Retinal Ganglion Cell Spiking Activity in the Mouse

Introduction.......................................................... 48

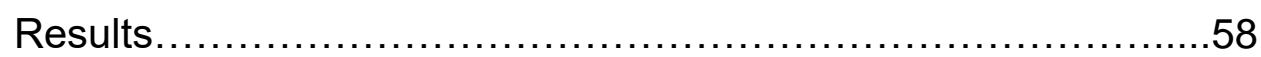

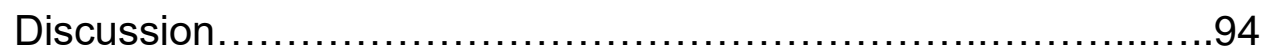

Chapter IV . . . . .

TRPV1 is Expressed in Specific Amacrine and Ganglion Cell Classes in the Mouse Retina

Introduction.................................................................

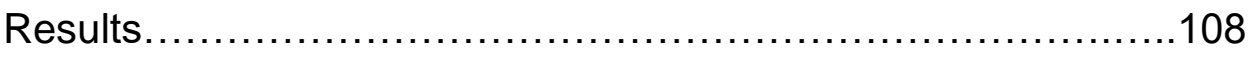

Discussion....................................................... 131

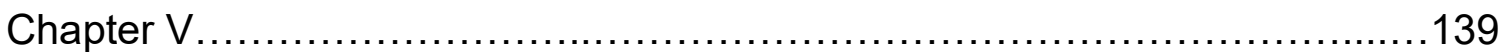

LRIT3 Regulates TRPM1 Localization and OPL Synaptic Structure and Function

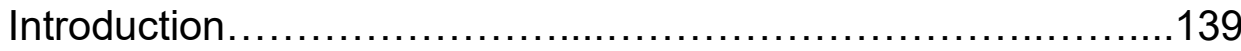


Results............................................................ 144

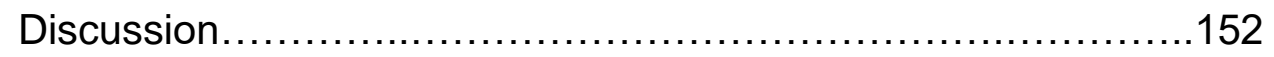

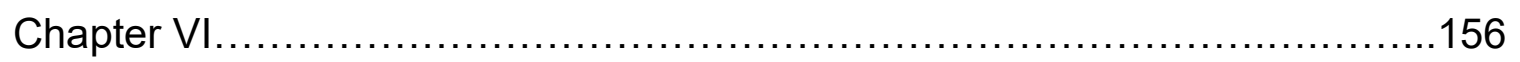

General Discussion and Future Directions

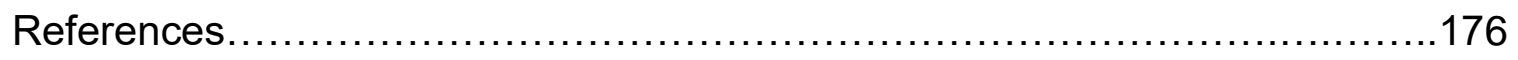

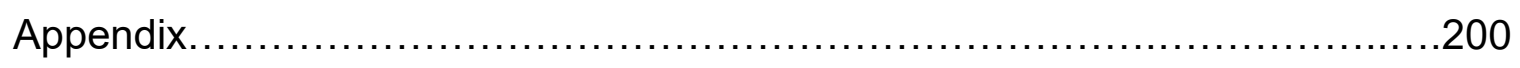

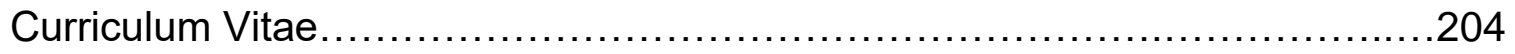




\section{LIST OF FIGURES}

FIGURE

PAGE

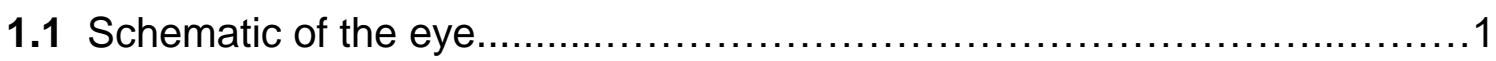

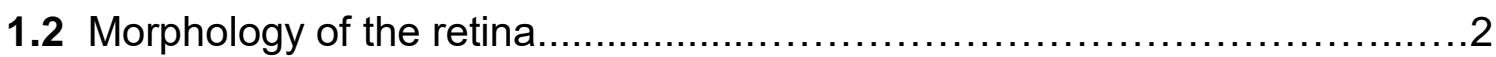

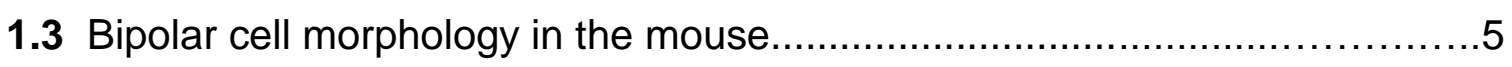

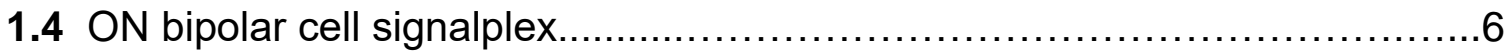

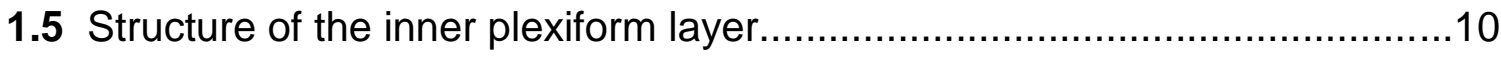

1.6 Mechanisms of feedback and feedforward inhibition .......................12

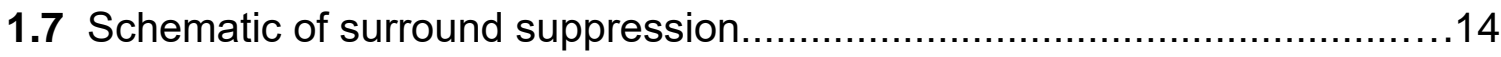

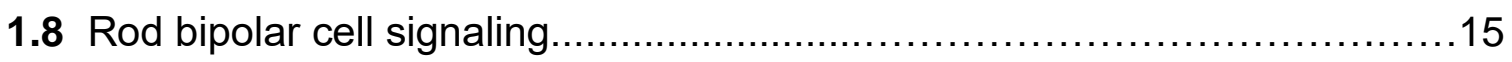

1.9 ACs inhibit other ACs in serial inhibition....................................16

1.10 GCs can have sustained or transient responses ........................17

1.11 Stratification depth in the IPL corresponds with temporal characteristics of GC spike rate in salamander retina...................................................

3.1 Higher luminance is required to evoke a response in $\operatorname{Trp} V 1 \% \ldots \ldots \ldots \ldots \ldots \ldots . . .59$

3.2 TRPV1 does not contribute to cone pathway signaling in GCs sampled in vivo

3.3 TRPV1 does not contribute to cone pathway signaling in GCs sampled in vivo. 62

3.4 Spontaneous activity is lower in TrpV1\% OFF GCs. 64 
3.5 Spontaneous activity is lower in TrpV1\%ON GCs and do not increase with light

3.6 OFF response durations were prolonged in $\operatorname{TrpV} 11-$ tOFF GCs to photopic stimuli .70

3.7 OFF response durations were prolonged in $\operatorname{TrpV1} 1-$ tOFF GCs to photopic stimuli. .71

3.8 Maintained response rates were increased in Trp $1 \%$ tOFF GCs compared to WT. .73

3.9 Maintained response rates were lower in $\operatorname{TrpV1} 1-\mathrm{ON}$ GCs compared to WT.

3.10 $\operatorname{TrpV1\% ON~GC~were~more~transient~than~WT~in~vivo.~}$ .78

3.11 WT sustained GC spontaneous activity was greater than TrpV1\% in vivo

3.12 Trp V1\% SOFF GCs peak and maintained firing rates were lower than WT. .81

3.13 Trp V1 $\%$ tOFF GC peak and maintained firing rates were similar to WT ....83

3.14 Trp V1 $\%$ ON GC peak and maintained firing rates were lower than WT ...85

3.15 $\operatorname{TrpV1}{ }^{-1-} \mathrm{tON}$ and $\mathrm{SON}$ GC maintained firing rates had inverse ARFs .87

3.16 TrpV1- tON GC peri-stimulus suppression had center summation and surround suppression. 89 3.17 Possible explanation for the peri-stimulus suppression of maintained rates in $\operatorname{TrpV} 1 \% \mathrm{ON}$ GC. .90 
3.18 TRPV1 enhanced the maintained response in ON center GCs with transient surround suppression

4.1 TRPV1 antibody label had a low signal to noise ratio in mouse DRG and retina 109

4.2 TdTomato is expressed in the INL, IPL \& GCL of TRPV1 ${ }^{\text {cre } / A i 9}$ retinas

4.3 TdTomato expression is similar in TRPV1 ${ }^{\text {cre/Ai9 }}$ and TRPV1 ${ }^{\text {cre }}-\mathrm{AAV}$ retinas 113

4.4 TRPV1 is expressed in mouse ACs 116

4.5 Neurotransmitter expression in TRPV1+ ACs. 118

4.6 TRPV1 is expressed in mouse GCs 120

4.7 Melanopsin is co-expressed in TRPV1+ M1 and M2 GCs 122

4.8 TRPV1 is expressed specific subsets of M1 and M2 ipGCs 125

4.9 TRPV1 agonist capsaicin evokes current in TRPV1+ ACs but not GCs in wholemount retina 128

5.1 Immunohistochemical and Western blot verification that Lrit3 expression is eliminated in the Lrit3 ${ }^{\text {emrgg1 }}$ mouse retina

5.2 ERGs from Lrit3\% mice express a no b-wave phenotype consistent with models of CCSNB 143

5.3 Responses of BCs to CPPG, Capsaisin, or Kainate puffs 145

5.4 Nyctalopin is absent in the Lrit3 ${ }^{-/}$retina. 147

5.5 Lrit3 is expressed at both rod and cone photoreceptor terminals in several mouse models of cCSNB. 149 
5.6 Visually-evoked responses of Lrit3 $^{-/}$GCs are significantly altered compared to $\mathrm{Grm6}^{-/}$and controls. 


\section{LIST OF TABLES}

TABLE

PAGE

2.1 Antibodies used to label specific proteins in the retinas of reporter mice....31

2.2 Antibodies used to label specific proteins in the retinas of $\operatorname{TrpM1}{ }^{-}, \mathrm{Ny} x^{n o b}$,

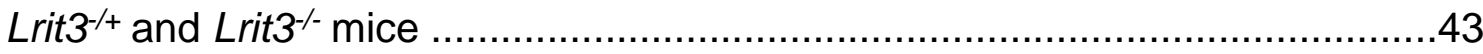

6.1 Mouse lines with fluorescently labeled GCs, which I crossed with

TrpV1-

6.2 Mouse lines with fluorescently labeled GCs, which can be crossed with

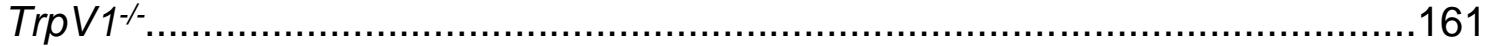




\section{CHAPTER I}

\section{INTRODUCTION}

\section{The Retina}

1. Structure of the retina

The retina is a neural structure lining the back of the eye (Fig. 1.1) containing photoreceptors that transduce light into electrical signals that encode

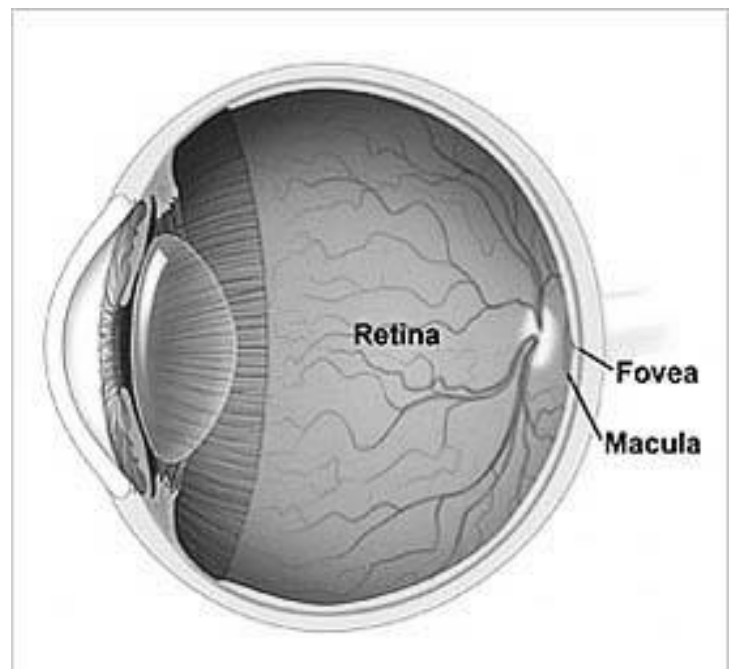

Figure 1.1 Schematic of the eye. The retina lines the back of the eye. Light enters the eye through the cornea and pupil. The lens focuses the light on the retina which lines the back of the eye. (Phillips Eye Specialists, NJ) the visual environment. This

signal is the basis for all

subsequent processing in the

rest of the visual system.

The retina consists of

three layers of cells and two

plexiform layers, in which cells

make synaptic connections

(Fig. 1.2). The outer nuclear

layer (ONL) is composed of

photoreceptors. Light is

transduced by photoreceptors

via decreased glutamate release from their terminals. This initiates a response in bipolar cells (BCs) or horizontal cells (HCs) in the inner nuclear layer (INL). The 
output of the BCs drives both amacrine cells (ACs), and retinal ganglion cells

(GCs). These INL cells integrate and modulate the primary excitatory signal

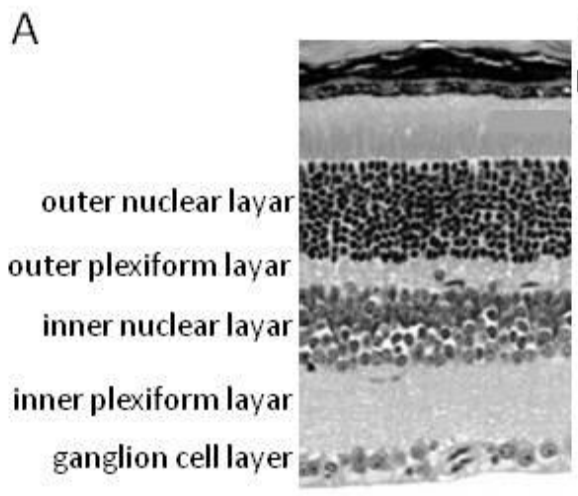

B

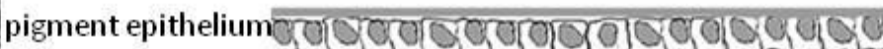

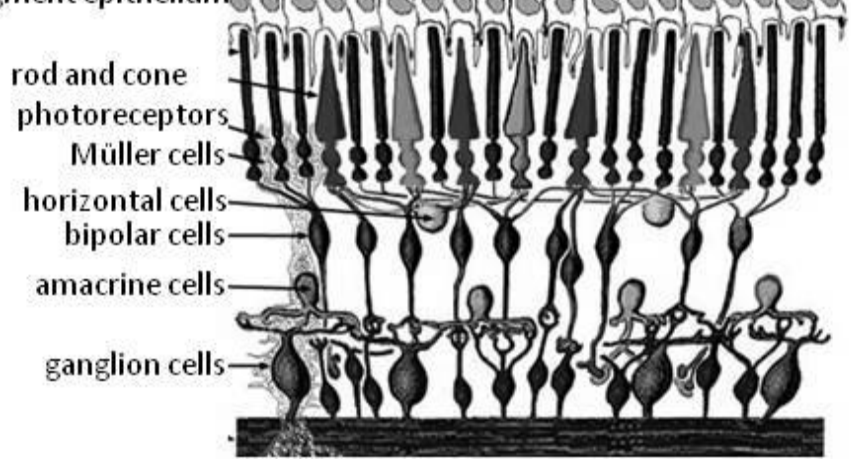

Figure 1.2 Morphology of the retina A) A transverse section of the retina in which cell bodies are labeled with hematoxylin and eosin stain ("DeltaBase Histology Atlas," 2000-2006). The retina is organized into three nuclear layers with two plexiform layers between where cells make synaptic connections B) The retinal pigment epithelium abuts the rod and cone PR outer segments. The outer nuclear layer is composed of rod and cone cell bodies. The inner nuclear layer is composed of horizontal cells, bipolar cells, amacrine cells, and Müller cells (MCs). The GCL is composed of GCs and displaced ACs (Kolb, 2011)

from the photoreceptors through BCs to GCs in the ganglion cell layer (GCL). The axons of the GCs, which lie on the vitreal side of the GCL, form the nerve fiber layer and exit the eye to form the optic nerve (see Kolb 2011 for review of retinal anatomy). The optic nerve projects to specific subcortical nuclei including the lateral geniculate nucleus, superior colliculus, olivary pretectal nucleus and suprachiasmatic nucleus (Purves et al., 2001).

2. The function of the retina

2.1 The primary excitatory pathway 
2.1.1 Rod and cone photoreceptors initiate vision and establish parallel pathways

Photoreceptors have distinct morphology related to their function. Their outer segments are comprised of stacked discs composed of membranes in which opsin molecules are embedded. The retinal chromophore is bound to opsin. When 11-cis-retinal absorbs a photon it isomerizes to the all-trans state. Individual photons absorbed by the opsin molecules cause a cis to trans conformational change in the retinol protein and subsequent g-protein transduction cascade activation (Hecht et al., 1942; Dowling 1987; Schneeweis \& Schnapf, 1995; Hargrave and McDowell, 1992; Fu, 2010 for review). Unlike most typical neurons, the unstimulated photoreceptor is depolarized in the dark due to constitutively open cation channels in the outer segments. As a result, photoreceptors constantly release glutamate in the dark. Light results in hyperpolarization of the photoreceptor and a reduction in glutamate release (Chabre, 1985; Hargrave \& McDowell, 1992; Kolb, 2011).

The rod and cone photoreceptors establish the first parallel pathway through the retina to initiate signaling under low and high luminance conditions, respectively. There are on average 4.6 million cones and 92 million rods in the human ONL (Curcio et al., 1990). Cones are responsible for vision under high luminance conditions and mediate high resolution vision. In humans, the highest density of cones is found in the central fovea which is also devoid of rods and other cells. The high resolution vision that humans use for reading is enabled by the functional anatomy of the fovea and surrounding macular regions. A single 
foveal cone sends information through the BCs in the INL to 1 to 3 ganglion cells (Ahmad et al., 2003) in a high resolution construct. In mice, the cones make up approximately $1 \%$ (Jeon et al., 1998) to 3\% (Carter-Dawson \& LaVail, 1979) of the photoreceptors and are evenly distributed across the central and peripheral retina (Carter-Dawson \& LaVail, 1979). The rod pathway is responsible for our low resolution night vision. Rods saturate at lower light intensities (all rhodopsin becomes activated) than cones. A large number of rods converge onto each rod bipolar cell (RBC), several of which connect with a single GC. This convergence and the innate amplification properties of the G-protein-mediated cascade within the rods make the rod pathway highly sensitive (Stryer, 1991; Yau, 1994), but with poor spatial resolution. RBCs do not synapse directly onto GCs but transfer information through the All $A C$ to cone bipolar cell $(C B C)$ terminals which synapse directly with GCs (Kolb \& Famiglietti, 1974; Famiglietti \& Kolb, 1975; Strettoi et al., 1990; Strettoi et al., 1992; Chun et al., 1993; Kim et al., 1998).

\subsubsection{The Photoreceptor to Bipolar Cell Synapses establish parallel ON} and OFF pathways

In the mouse there are 10 morphological types of $\mathrm{BCs}-1 \mathrm{RBC}$ and $9 \mathrm{CBCs}$ (Fig. 1.3; Ghosh et al., 2004). While rod photoreceptors hyperpolarize in response to light, RBCs and approximately half of the CBCs depolarize and are called ON BCs. This is due to the sign inverting synapse between the photoreceptor the ON BC. The G-protein coupled metabotropic glutamate receptor-6 (mGluR6) is expressed in the dendritic tips of the ON BCs (Fig. 1.4; Masu et al. 1995; Vardi et al. 2000). Binding of glutamate to mGluR6 activates 
the $\mathrm{Ga}_{\circ} \mathrm{g}$-protein mediated cascade. The activation of $\mathrm{Ga}_{\circ}$ results in the closure of the ion channel, TRPM1 (Dhingra et al., 2000;

Sampath and Rieke, 2004; Snellman et al., 2008; Bellone et al, 2008; Shen et al., 2009; Morgans et al., 2009; van Genderen et al., 2009, Koike et al., 2010;

Peachey et al., 2012). In response to light, the photoreceptors hyperpolarize and reduce glutamate release and the subsequent activation of mGluR6. $\mathrm{Ga}_{\circ}$ becomes inactivated and TRPM1 opens, resulting in depolarization of the ON-BC (Vardi et al. 1993; Dhingra et al. 2000). Ga o inactivation by hydrolysis of bound

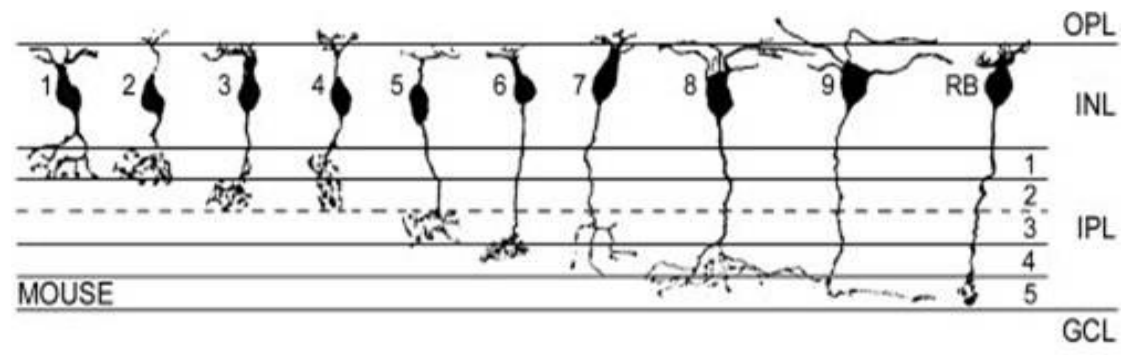

Figure 1.3 Bipolar Cell Morphology in the Mouse. There are nine morphological classes of CBCs and 1 type of RBC in the mouse retina (Ghosh et al., 2004). OFF CBCs terminate in sublaminae 1-2 while ON CBCs and RBCs terminate in sublaminae $3-5$.

GTP is accelerated by Regulators of G-protein Signaling (RGS) 7 and 11 (Cao et al., 2012; Shim et al., 2012). GPR179 interacts with TRPM1 and is required for expression of RGS7 and RGS11 in the dendritic tips of ON BC (Orlandi et al., 2012; Ray et al., 2014). In the absence of GPR179 the sensitivity of the cascade and TRPM1 is decreased. Nyctalopin and LRIT3 are both leucine rich repeat proteins which are necessary for the proper localization of TRPM1 in the dendritic tips of the ON BCs. In the absence of LRIT3, other significant cascade proteins are absent from the $\mathrm{ON}$ cone $\mathrm{BCs}$ as well. The unidentified protein on 
the cone terminals which is labeled by peanut agglutinin (PNA) is also absent

(Neuillé et al., 2015). We investigate the functional implications stemming from

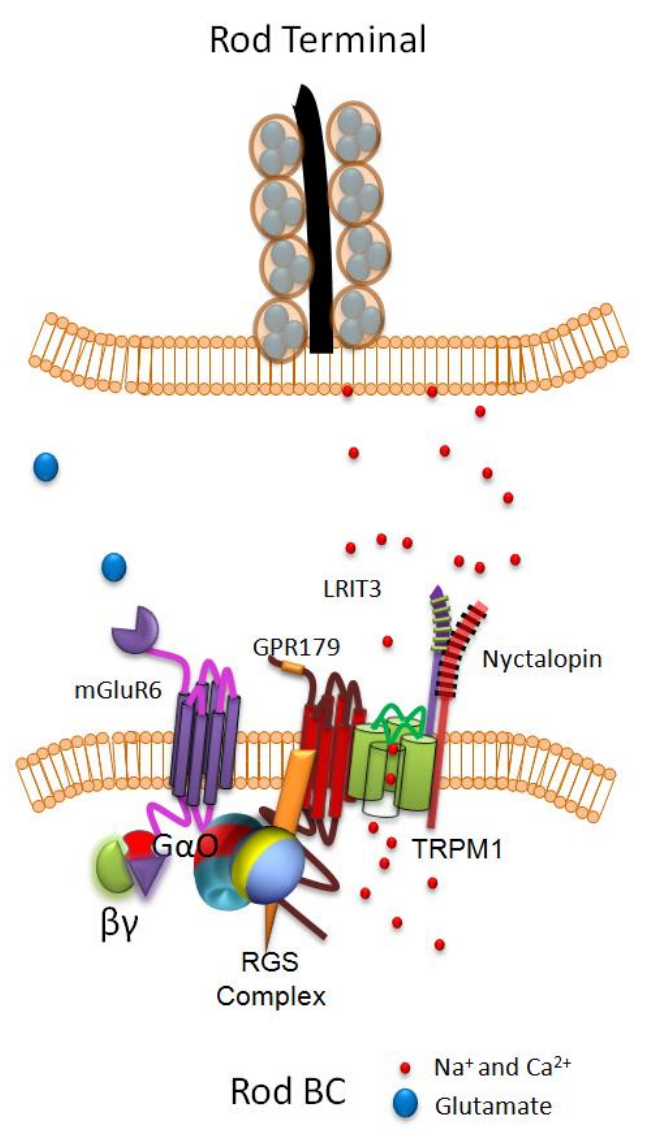

Figure 1.4 ON Bipolar Cell Signalplex. TRPM1 is associated with the metabotropic glutamate receptor mGluR6 and G-proteins Gao and Gßy. They and multiple accessory proteins form a signaling complex (signalplex) in ON BCs, which mediates transmission of visual signal at the synapse. In the dark, glutamate is continuously released from rod and cone photoreceptors and binds to the mGluR6 receptors on the post-synaptic ON BC dendrites. Once bound, a G。 g-protein coupled cascade is activated, which, through an unknown process, closes the TRPM1 cation channel. In response to light, glutamate release is decreased and mGluR6 is inactivated. The regulator proteins G $\beta 5$, RGS7 and RGS11, as well as GPR179 and R9AP, mediate the rapid inactivation of the mGluR6 cascade and opening of the TRPM1 channel. Influx of $\mathrm{Na}^{+}$and $\mathrm{Ca}^{2+}$ through the non-specific TRPM1 cation channel depolarizes the ON BC. Accessory proteins nyctalopin and LRIT3 are critical for the proper localization TRPM1 in the tips of the ON BC dendrites. the loss of LRIT3. The Off

pathway starts with the OFF

BCs which express ionotropic

glutamate receptors (iGluR) on

AMPA/Kainate cation channels

at the dendritic tips post-

synaptic to the cone terminals

(Slaughter and Miller, 1985;

Dowling, 1987). However,

recent evidence suggests only

Kainate receptor/channels are

expressed in mouse OFF BCs

(Borghuis et al., 2014). Since

Kainate channels open directly

in response to glutamate

binding, these cells depolarize

to light offset like the

photoreceptors in a sign-

conserving mechanism

(Werblin, \& Dowling, 1969;

Euler et al., 1996). ON CBCs

terminate in the IPL closer to 
the GCL (sublamina 3-5) than OFF CBCs, which terminate in sublamina 1 and 2 close to the INL (Fig. 1.3).

\subsubsection{Retinal Ganglion Cells integrate all retinal inputs and provide the} basis for visual function

GCs are the output cells of the retina that send the coded information to the brain as action potentials or spikes (e.g., superior colliculus, lateral geniculate nucleus and other subthalamic nuclei) where the information is integrated and sent along to other visual areas or other sensory systems. GCs have been divided into 17-30 morphological classes in the mouse retina based on size and shape of their somata, dendritic structures and lamination pattern in the IPL (Sun et al., 2002; Badea \& Nathans, 2004; Kong et al., 2005; Coombs et al., 2006). Functionally, GCs have been categorized in cat and primate retinas based on their light responses. Different ganglion cells extract distinct visual information. For example, GCs are classified as ON, OFF or ON/OFF, corresponding to the luminance step that produces their excitatory response (Enroth-Cugell \& Robson, 1966; Boycott \& Wassle, 1974). In addition, GCs can respond either transiently to the onset or offset of the luminance step or in a sustained manner for the duration of the presence of the stimulus (Ikeda \& Wright, 1972). In mice, genetically distinct GCs have been categorized morphologically and functionally (see Sanes and Masland, 2015 for a review). Approximately half of mice GCs have been classified in this way and evidence thus far shows a similar pattern to the cat and primate (Sagdullaev \& McCall, 2005; van Wyk et al., 2009; Hong et al., 2011, Yee et al., 2012, Sanes and Masland, 2015). 


\subsubsection{Intrinsic photoreceptive retinal ganglion cells}

There is another class of photoreceptors in the retina. A population of GCs expresses the photo pigment melanopsin, which makes them intrinsically photosensitive (ip) GCs (Foster et al., 1991; Provencio et al., 1998; Berson et al., 2002). In the absence of rod or cone input, humans and mice detect irradiance through ipGCs which project to nuclei in the brain responsible for both non-image forming functions such as regulating circadian rhythms (Foster et al., 1991; Herzog, 2007; Czeisler et al., 1995) and pupil constriction, (Hattar et al., 2006) as well as image-forming functions for pattern vision (Hattar et al., 2006; Ecker et al., 2010; Schmidt et al., 2011). There are several morphological and functional classes of ipGCs. M1 GCs were the first discovered (Berson et al., 2002) and express the most melanopsin and subsequently have the largest melanopsin light responses. M1 GCs are divided into two classes based on expression or absence of the transcription factor Brn3b and their projections to either the suprachiasmatic nucleus (SCN) where they regulate circadian rhythms (Brn3b negative) or the shell of the olivary pretectal nucleus (OPN; Brn3b positive) where they regulate pupil constriction (Hattar et al., 2006). M2, ON GC with widespread sparse processes, project to the dLGN, SC, and core of the OPN. Three other classes of ipGCs have been identified to date, one bistratified (M3), and two ON (M4 and M5) all of which most likely project to the dLGN and SC (Schmidt et al., 2011).

\subsection{The primary inhibitory pathways}


Y-Aminobutyric acid (GABA) and glycine are the major inhibitory neurotransmitters of the central nervous system. Their release causes chloride channels to open and leads subsequently to membrane hyperpolarization. HCs and $\mathrm{ACs}$ are the major inhibitory cells in the retina.

\subsubsection{Amacrine Cells}

There are 27 morphological types of ACs and these have been best characterized in the rabbit retina (MacNeil et al., 1999). Although the mouse retina is less well studied, it appears to share most, if not all, of the types described in the rabbit. ACs can be divided equally into cells that express GABA or glycine as their primary neurotransmitter (Pourcho \& Goebel, 1983; Mosinger

et al., 1986; Crooks \& Kolb, 1992; Menger et al., 1998; Haverkamp and Wässle, 2000). Certain types of ACs also contain other neurotransmitters or neuromodulators; for example, choline acetyl-transferase (ChAT) found in cholinergic cells or tyrosine hydroxylase $(\mathrm{TH})$ found in dopaminergic cells can be used to label specific types of ACs (Versaux-Botteri, et al., 1984; Crooks \& Kolb, 1992). Also, antibodies to specific calcium binding proteins like calbindin, calretinin and parvalbumin label specific AC types (Fig. 1.5). These proteins and many others have been used to look at the organization of the IPL (Pochet, et al., 1991; Haverkamp and Wassle, 2000).

\subsection{1.a Organization of the IPL}


The axons of BCs terminate and form connections with GCs and ACs in specific strata within the IPL that are correlated with their visual response properties. Cells that stratify in lamina A and B respond to the offset and onset of

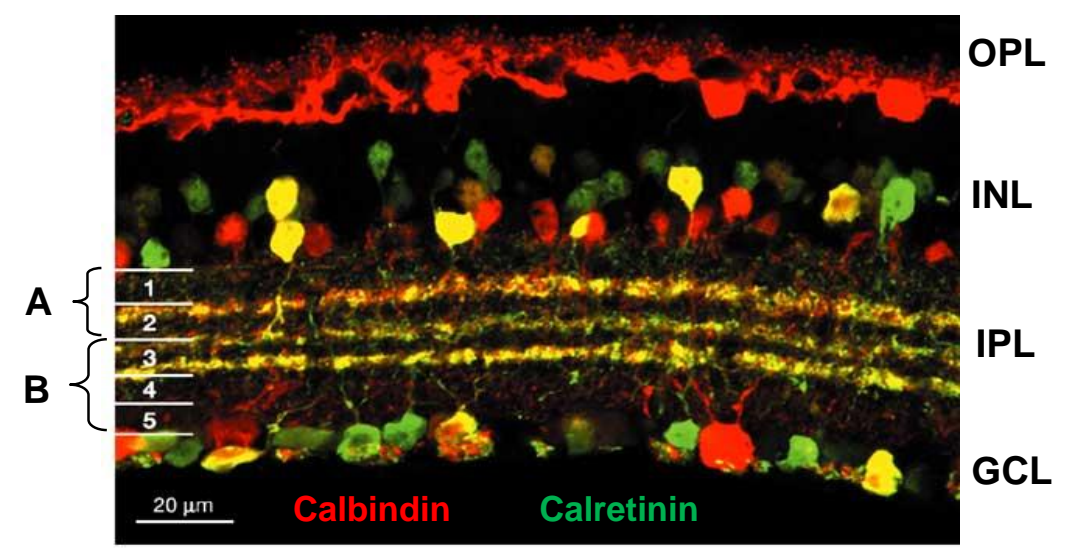

Figure 1.5 Structure of the inner plexiform layer. Calbindin (red) and calretinin (green) expressing cells have processes that terminate in specific sublaminae of the IPL. ACs in the INL and dACs and GCs in GCL terminate in three distinct layers, 2 in lamina $A$ and 1 in lamina B. GCs and BCs which terminate in lamina $A$ and $B$ depolarize to light decrements and increments respectively (Wassle, 2004)

light respectively (Fig. 1.5). The IPL is further divided into 5 substrata with 1 and 2 in the OFF lamina A and 3-5 in ON lamina B (Pochet, et al., 1991; Haverkamp and Wassle, 2000; Ghosh et al., 2004). Markers for specific cell types can be used to delineate the layers as shown with calretinin staining (Fig. 1.5; Wassle, 2004). All functionally classified ON or OFF GCs are monostratified in lamina B or A respectively. All ON-OFF GCs are bistratified in both A and B sublamina. BCs can stratify in more than one sublamina, but only within the same lamina, which also corresponds to the visual responses. ACs can be mono-, bi-, or multistratified and morphology and function are also correlated.

2.2.1.b. AC morphology 
ACs have been classified based on the neurotransmitter that they use (glycine or GABA) and the morphology of their dendrites. There are four main classes based on dendritic field diameter: narrow-field (30-150 um), small-field (150-300 um), medium-field (300-500 um) and wide-field (>500 um) (Kolb et al., 1981). An extensive morphological survey of ACs has been conducted in the rabbit retina (MacNeil et al., 1999). The narrow-field ACs are the largest class and can be divided into the broadly or narrowly stratifying subclasses, which stratify in more than two or one to two sublaminae respectively. There are ACs in the GCL known as displaced ACs (dACs). Similarly, Müller et al., (2007) conducted a morphological survey and classified 10 types in the mouse: 4 medium-field (50 - $200 \mu \mathrm{m})$ and 6 wide-field $(>1 \mathrm{~mm})$ and Lin and Masland (2006) conducted a survey of displaced amacrine cells in the mouse.

\subsection{1.c. AC function}

ACs transmit inhibitory information primarily within the IPL of the retina, this input appears to modulate visual signals that are transmitted via the excitatory vertical pathways through the retina. The next sections discuss some of the ways ACs extract this information to create the GC neural code:

\section{i. Feedback and feedforward inhibition}

Feedback inhibition is thought to decrease the amplitude and duration of GC receptive field (RF) center responses (Fig. 1.6; Zhang et al., 1997; Eggers et al., 2007). One such circuit involves the $G A B A_{c}$ receptors located nearly 
exclusively on the terminals of ON BCs (Lukasiewicz and Shields, 1998). Increments of light depolarize the ON BCs causing them to release glutamate. Synaptically connected GABAergic ACs are depolarized by glutamate and subsequently release GABA back onto the $\mathrm{ON} \mathrm{BC}$ terminals, decreasing subsequent output to the ON GCs (Fig. 1.6; Zhang et al., 1997; McCall et al., 2002). Subsequently, ON GCs in mice lacking GABAc receptors have increased

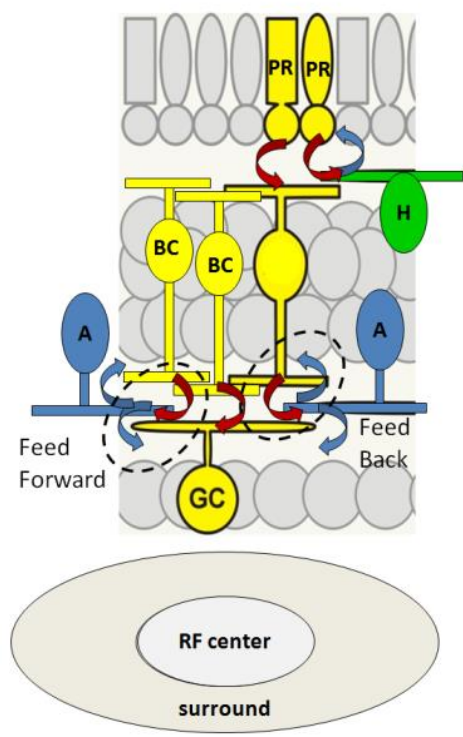
spontaneous and light evoked spiking rates compared to WT (Lukasiewicz et al., 2004; Sagdullaev et al., 2006). GABA ${ }_{c}$ mediated inhibition in the RBCs is larger and slower than GABAA mediated inhibition (Lukasiewicz et al., 2004). Therefore, $\mathrm{GABA}_{c}$ mediated feedback inhibition onto the $\mathrm{BC}$ terminals shapes temporal components of ON GC responses as well (Dong and Werblin, 1998; Sagdullaev et al.,

Figure 1.6 Mechanisms of feedback and feedforward inhibition. ACs receive excitatory glutamate input from BCs and subsequently release inhibitory neurotransmitter back onto the BC terminal (feedback) or forward onto the post-synaptic GC (feedforward) to regulate spatial and temporal properties of $\mathrm{GC}$ responses 2006).

Feedforward inhibition occurs when ACs are activated by release of glutamate from activated BCs and subsequently inhibit post-synaptic GCs (Fig. 1.6). Feedforward inhibition may aid in detecting fast changes in luminance values for high resolution vision (Russell and Werblin, 2010). Feedforward inhibition can be mediated by both GABA and glycine (Nobles et al., 2012). 


\section{ii Center-surround inhibition}

Lateral inhibition from cells outside the GC receptive field center is a type of feedforward inhibition. Inhibition from horizontal cells and ACs onto excitatory cells in both plexiform layers forms the center-surround organization of the receptive field (Fig. 1.7; Werblin \& Dowling, 1969; Cook \& McReynolds, 1998). Many neurons, including GCs have an RF center-surround organization. The RF is defined as the spatial area in which stimulation can elicit a response (Werblin \& Dowling, 1969). The RF center size is correlated with the dendritic field of the cell and its excitatory inputs from the BCs. Stimulation of the entire RF field center evokes the largest excitatory response and stimuli smaller than the RF center evoke a proportionally smaller response. When the stimulus extends outside the dendritic field spread and activates neighboring inhibitory cells, the excitatory response due to RF center stimulation is diminished (Fig. 1.7; Barlow, 1953, Rodieck \& Stone, 1965; Enroth-Cugell \& Lennie, 1975). Generally, the RF surround inhibition is shaped by GABA and the ACs that mediate it are the widefield ACs (Protti \& Llano, 1998; Hartveit, 1999; Flores-Herr et al., 2001; O’Brien et al.,2003; Roska et al., 2006). In contrast, local feedforward inhibition onto the RF center is mediated by narrow-field glycinergic ACs (Menger et al., 1998; O'Brien et al., 2003; Nobles et al., 2012).

The development of retinal morphology including the mosaic tiling of distinct GC classes is mature by eye opening, which is approximately P12-P14 in the mouse (Anishchenko et al., 2010). At P10, the retina has segregated into three neuronal layers and excitatory inputs, via the parallel ON and OFF 
pathways to the GCs,

are connected and

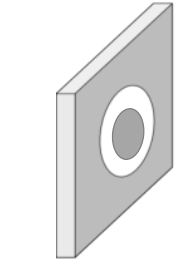

Suppressive Peak

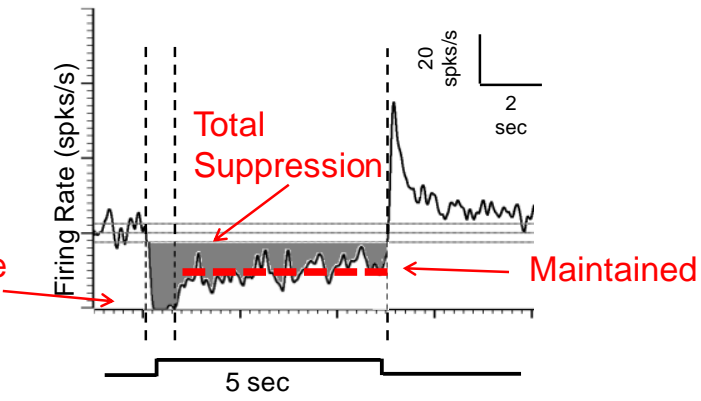

Surround Antagonism = recruitment of inhibitory inputs

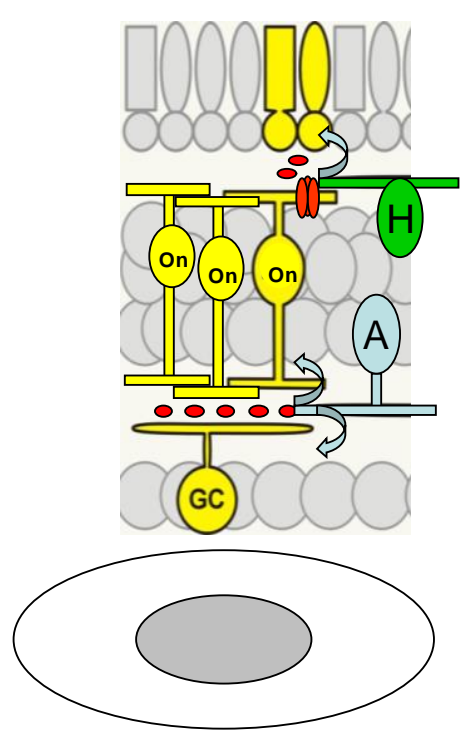

Figure 1.7 Schematic of surround suppression. Inhibitory signal from AC and horizontal cell form the surround suppression of the GCs which can be isolated and analyzed using annular stimuli that stimulates only the surround. functional. The GC

RFs are established

and, while their

dendritic processes will

expand laterally

(Morgan et al., 2008),

the functional RF

center gets smaller

and more

synchronized by

maturity

(Anishchenko et al., 2010; Cantrell et al., 2010; Koehler et al., 2011). Because the RF surround extends beyond the RF center, response suppression

can be evoked and RF surround contributions isolated and measured using an annular stimulus (Fig. 1.7), which only stimulates the RF surround.

iii. Cross-over inhibition 
RBCs do not make direct synaptic connections with GCs but synapse with All ACs. These narrow field bistratified ACs then transfer the signal to ON CBC axon terminals through gap junctions (Fig. 1.8). All ACs simultaneously inhibit

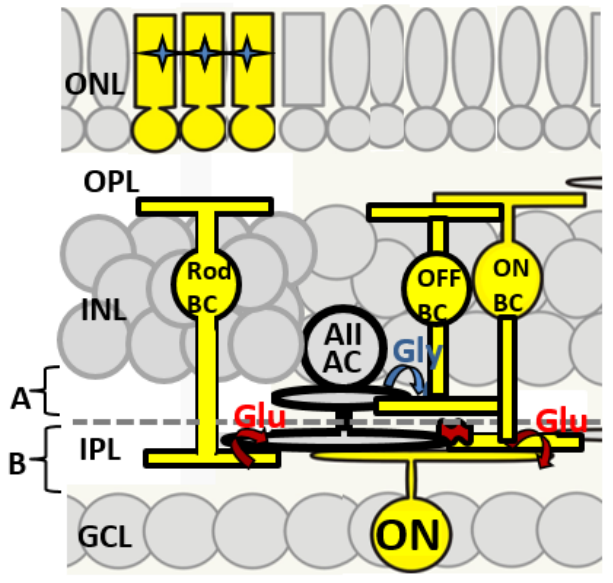

Figure 1.8 Rod bipolar cell signaling. RBCs do not activate ON GCs directly but through the All AC which is coupled to ON CBCs with gap junctions. All ACs simultaneously inhibit OFF CBCs and GCs through glycinergic synapses
OFF CBCs through glycinergic synapses on their axon terminals. Therefore, an increment of light causes an excitatory response in $\mathrm{ON}$ GCs and also causes a suppression of the response in OFF GCs

from cross-over inhibition (Manookin et al., 2008; van Wyk et al., 2009; Chen et al., 2010; Liang and Freed, 2010). Cross-over inhibition can be initiated in ON or OFF lamina and provides inhibition to the opposite pathway. Glycinergic narrowfield cells that are stratified in both ON and OFF sublamina mediate cross-over inhibition (Werblin, 2010). Cross-over inhibition is thought to enhance the GC evoked spiking activity by reducing inhibition on the GC (Zhang \& McCall, 2012)

iv. Serial inhibition

ACs can also inhibit other ACs through serial inhibition (Fig. 1.9; Zhang et al., 1997; Eggers \& Lukasiewicz, 2010; Russell \& Werblin, 2010 Anderson et al., 2011; Nobles et al., 2012). In this way, an increase in inhibitory neurotransmitter release from one cell will lead to a decrease in inhibitory neurotransmitter release in a serially connected cell. For example, the $\mathrm{GABA}_{c}$ mediated feedback circuit 
mentioned above is itself inhibited by a second AC that receives excitatory input from $B C s$ and releases $G A B A$ onto the $G A B A_{A}$ receptors of the feedback $A C$ (Zhang et al., 1997). The feedback AC is subsequently inhibited from releasing

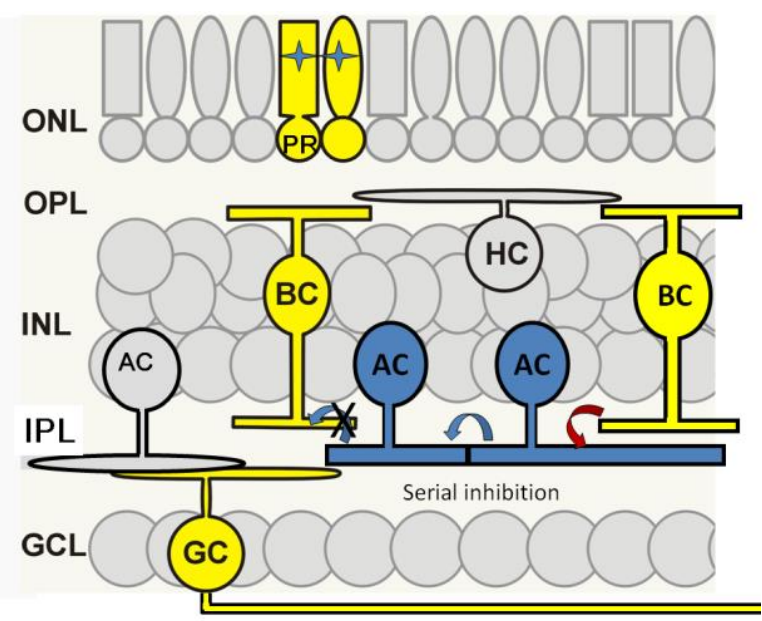

Figure 1.9 ACs inhibit other ACs in serial inhibition.
GABA back onto the BC. The

interplay of these inhibitory

signals is thought to regulate

the duration of the visual

response - the feedback $A C$

shortens the duration of the

response and the serial $\mathrm{AC}$

prolongs it (Eggers \&

Lukasiewicz, 2010; Russell \&

Werblin, 2010; Nobles et al., 2012). Studies of cell networks in the retina reveal many concatenated circuits involving glycinergic and GABAergic ACs (Anderson et al., 2011). One well studied example of this is the RBC to A17 synapse. RBCs form a dyad synapse with the glycinergic All and GABAergic A17. The A17 forms a reciprocal feedback synapse onto the $\mathrm{RBC}$, releasing GABA onto $G A B A_{c}$ and $\mathrm{GABA}_{\mathrm{A}}$ receptors (Grimes et al., 2015). This shapes the time course of glutamate release from RBCs (Dong \& Hare, 2003; Singer \& Diamond, 2003; Chavez et al., 2010). The A17 AC also receives GABAergic inhibition from a spiking wide-field $A C$ which mediates serial disinhibition of the RBC (Zhang et al., 1997; Roska et al., 1998; Eggers \& Lukasiewicz, 2006, 2010). Serial inhibition may be activated by wide or full-field stimuli whereas direct inhibition is activated by narrow stimuli directly over the RBC (Eggers \& Lukasiewicz, 2011). Nobles 
and colleagues (2012) report a role for glycine receptors in mediating serial inhibition to ON BCs or GCs.

\subsection{Temporal parallel pathways (transient / sustained)}

The presence of a maintained light stimulus produces a sustained membrane hyperpolarization in rod and cone photoreceptors (Sampath et al., 1999; Fain et al., 2001) and leads to a sustained inhibition in glutamate release (Fig. 1.10;

Chabre, 1985; Hargrave \& McDowell, 1992). RBCs also show sustained
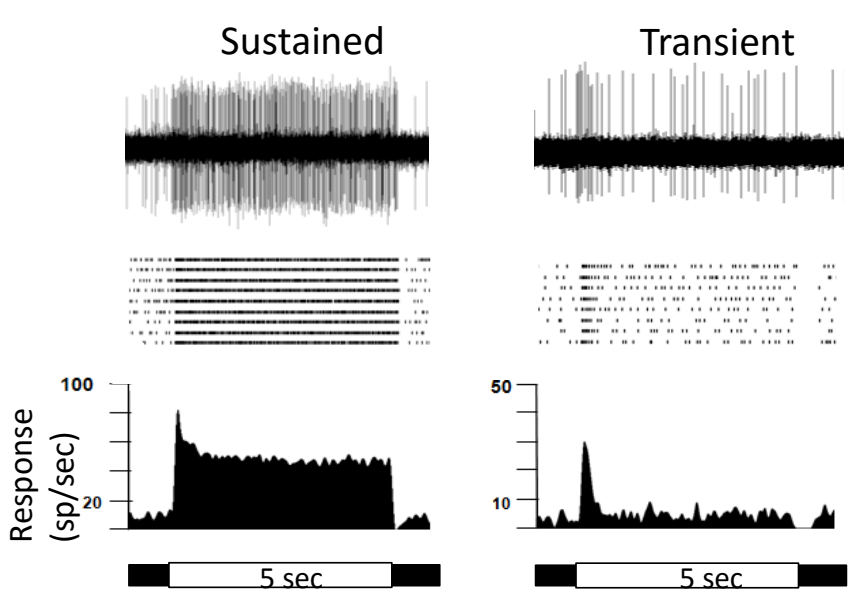

Figure 1.10 GCs can have sustained or transient responses. Sustained cells maintain an increased spike rate for the entire duration of a stimulus while transient cells return quickly to spontaneous rates. Shown are spike trains to a stimulus, raster plots for 10 trials and average peristimulus time histograms (PSTHs) showing spikes/sec over time in response to a $5 \mathrm{sec}$ light spot. depolarizations in the presence of a maintained light stimulus. In contrast, ON CBCs can be divided into those that are sustained and those that are transient (Awatramani and Slaughter, 2000). In ground squirrel there are three distinct functional types of OFF CBCs each

with a different AMPA or Kainate ionotropic glutamate receptor. OFF CBCs with AMPA receptors have more transient responses to maintained light than the two types expressing kainate receptors (DeVries, 2000). However recent evidence suggests that in the mouse, OFF CBCs only have kainate receptors (Borghuis et al., 2014). ON and OFF GC responses can also be categorized as sustained or 
transient. Sustained GCs have maintained EPSCs to steady state stimulus (Roska et al., 2000), and maintain an increased spike rate that matches the temporal profile of their stimulus (Werblin et al., 2001). Transient GCs respond with a short burst of excitatory activity that quickly returns to the spontaneous spiking level (Fig. 1.9). In the ground squirrel, transient OFF BCs terminate on transient OFF GCs (DeVries, 2000) and in salamander retina transient ON-OFF GCs are post-synaptic to transiently depolarizing CBCs (Awatramani and Slaughter, 2000). This suggests that the primary excitatory pathway is responsible for the response duration of GCs. AC inhibition also tunes the duration of GC responses. Dong and Werblin (1998) showed that feedback inhibition mediated by GABAergic AC connections at the BC terminal shortened the duration of glutamate release and resulted in transient GC responses.

Besides the lamination organization of ON and OFF pathways in the IPL, the level of stratification in the IPL also delineates temporal response properties (Fig. 1.11; tiger salamander - Awatramani and Slaughter, 2000; Ground squirrel DeVries, 2000; Werblin et al., 2001; mouse - Van Wyk et al., 2009; Borghuis et al., 2013; Baden et al., 2013; see Euler et al., 2014 for a review). BCs and GCs that stratify towards the middle of the IPL (sublamina 2-3) respond more transiently whereas those stratifying at its outer edges (sublamina1 \& 5) tend to be more sustained (Fig. 1.11; Werblin et al., 2001). Unlike the substantial literature on the morphological basis of ON and OFF pathway segregation, this idea of stratified temporal processing remains to be further tested. 
In the mouse, the center-surround organization is established simultaneously with excitatory input at P10 (see Center-surround lateral inhibition). In contrast, GC lamination pattern of the dendritic processes in the IPL

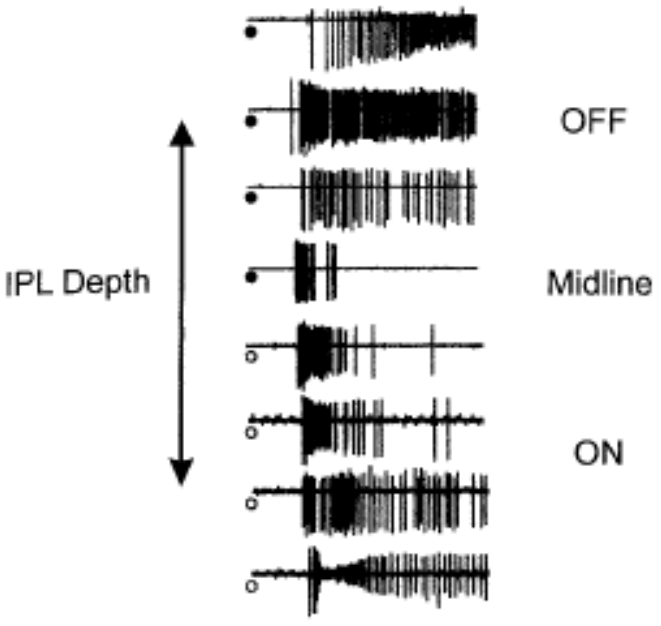

Figure 1.11 Stratification depth in the IPL corresponds with temporal characteristics of GC spike rate in salamander retina. (Werblin et al., 2001)

segregation of processes into proper IPL sublaminae and this process depends on light-evoked glutamate activity (GCs of dark reared mice do not segregate). By maturity only $20 \%$ of GCs have ON-OFF responses and bistratified morphology (Sun et al., 2002; Tian and Copenhagen, 2003). Aside from the reports of early ON/OFF responses, that by definition must be transient, the development of transient or sustained response properties has not been studied in any species.

\section{TRPV1}

1. TRP Channels are immature at P10. Many

GC dendrites are bistratified

and have not yet segregated

into their final sublamination

patterns. Functionally the

majority of cells have transient

ON-OFF responses (Tian and

Copenhagen, 2003). From P10

to $\mathrm{P} 30$, there is gradual 
The most recently discovered family of ion channels are the transient receptor potential (TRP) channels. The discovery of the first TRP channel was in mutant drosophila (Cosens, 1969) which had transient retinal responses to maintained light stimulation. There are 28 known TRP channels in mammals which are divided into six sub-families: canonical (TRPC1-7), vanilloid (TRPV16), melastatin (TRPM1-8), ankyrin (TRPA1), polycystin (TRPP1-3) and mucolipin (TRPML1-3) (Clapham et al., 2012). In the peripheral nervous system (PNS), TRP channels are involved in thermal sensation, nociception and tissue inflammation (Patapoutian et al., 2009 for review) but are also polymodal responding to a variety of stimuli including temperature, osmolality, chemicals, protons, and pressure or force - and function in a variety of sensory receptors. TRP channels are widely distributed in the CNS but their function there is not well studied. Several are the activating channels linked to mGluRs, such as TRPM1 in the retina ([see introduction]; Moran et al., 2004; Ho et al., 2012). In the hippocampus, there is evidence that TRPs are involved in neurite growth (Greka et al., 2003). Gilliam and Wensel (2011) confirmed the mRNA from all 28 TRP channels in the retina, however the function of only TRPM1 is well-understood: it is the signaling channel of all signals in ON BCs (introduction) and mutations in the gene are responsible for some forms of the human disease complete congenital stationary night blindness (Bellone et al., 2008; van Genderen, 2009). TRPC6 and TRPC7 may transduce signals in melanopsin-expressing retinal ganglion cells (Sekaran et al., 2007). TRPM7 \& TRPC1/3/6/7 channels are possible targets for regulating intracellular $\mathrm{Ca}^{2+}$ concentrations and subsequent neurotransmitter release from photoreceptors (Krizaj, 2012). TRPV4 activation by 
increased pressure modulates spike rate and has been shown to initiate pressure induced apoptosis of GCs in glaucoma (Ryskamp et al., 2011). The majority of understanding about the role of TRPV1 comes from research in the PNS and its role in pain.

\section{TRPV1 in the Peripheral Nervous System}

The peripheral nervous system (PNS) is comprised of the sensory and motor neurons that are not part of the brain and spinal cord. The sensory neurons of the PNS terminate in the skin and internal organs and have receptors that transduce a variety of external environmental stimuli into electrical activity, sometimes referred to as receptor potentials. Nociceptors convert painful or harmful stimuli and are found in nerve endings of large myelinated Aঠ fibers and small unmyelinated c-fibers (Perves et al., 2008). In particular, the TRPV1 receptors on the c-fiber afferents convey nociceptive signals to the spinal cord, which is transmitted to second order neurons in the spinal cord. These signals are then sent in specific pathways to the brain. Because c-fibers are small and unmyelinated, the conduction velocity is slow $(2 \mathrm{~m} / \mathrm{s})$ and gives rise to slow, burning pain after the initial sharp pain (Perves et al., 2004).

\subsection{TRPV1 Expression during development}

Using two reporter mice, Cavanaugh et al. (2011a) showed TRPV1 expression was highest during late embryonic development and subsequently declined in mature animals. In the dorsal root ganglion (DRG) which contains the cell bodies of the primary nociceptor afferents, TRPV1 expression reaches a 
peak at embryonic day 14.5 (E14.5) when approximately $66 \%$ of primary afferents express TRPV1. By adulthood, approximately 33\% of DRG cells express TRPV1 (Cavanaugh et al., 2011b). This observation of increased expression during development was confirmed in another TRPV1 reporter mouse line (Mishra et al., 2011).

\subsection{TRPV1 Function}

Activation of TRPV1 by capsaicin, noxious heat, or other stimuli opens a cation channel leading to depolarization, which causes the sensory neuron to spike. The brain interprets the signal as burning (Bevan \& Szolcsanyi 1990; Holzer 1991). A secondary result of the activation of TRPV1 induces the release of chemokines: neurokinin $A$, substance $P$ and calcitonin-gene-related-peptide (CGRP) that attract macrophages and bind to receptors in the surrounding tissue including endothelial cells and smooth muscle, leading to vascular dilation and leakage (Meng et al., 2009). This further increases the immune response, and induces swelling and redness that are hallmarks of the inflammatory response (Szallasi \& Blumberg 1999; Richardson et al., 2002 for review). Feedback pathways also sensitize TRPV1, lowering the threshold of response and increasing the probability of reactivation. This is accomplished by cAMP dependent PKA or $\mathrm{Ca}^{2+}$ dependent PKC phosphorylation of TRPV1 at different sites (Mandadi et al., 2004; Liu et al., 2005; Mohapatra et al., 2005). This process is responsible for the increased sensitivity and burning in inflamed tissue, in sunburned skin for example. $\beta$-arrestin-2 mediated dephosphorylation of TRPV1 results in desensitization (Por et al., 2012; Ho et al., 2012 for review). This 
pathway is particularly interesting as it may be a therapeutic target of pain relief without the unwanted side effects of hyperthermia associated with blocking TRPV1.

TRPV1 in the primary afferent central terminals (in the spinal cord) is also activated when the nociceptor is stimulated. Here it mediates $\mathrm{Ca}^{2+}$ influx and storage proportional to the size of the stimulus, thereby perhaps encoding nociceptive stimulus duration and intensity. This $\mathrm{Ca}^{2+}$ is stored in the mitochondria and slowly released into the intracellular solution, causing sustained calcium-dependent neurotransmitter release from the primary afferent, giving rise to postsynaptic asynchronous EPSPs that are maintained long after cessation of the stimulus (Medvedeva et al., 2009). Activation of TRPV1 leads to sustained neurotransmission in other systems and plays a role in chronic pain syndromes associated with arthritis, migraine and fibromyalgia (White et al., 2011 for a review).

\section{TRPV1 in the Central Nervous System}

\subsection{TRPV1 Expression}

Defining the expression of TRPV1 in the central nervous system (CNS) has been difficult due, in part, to its overall low level of expression. TRPV1 ${ }^{\text {nlacz-plap }}$ knock in reporter mice mentioned previously (Cavanaugh et al., 2011b) have been used to label cells that express TRPV1, using the reporter placental alkaline phosphatase (PLAP). This approach labels cells in nuclei that receive primary afferents from the spinal cord, such as the nucleus caudalis, solitary tract and nucleus ambiguus of the medulla, as well as the external lateral parabrachial 
nucleus and olfactory bulb. Staining also has been observed in other brain regions including the periaquaductal gray, hippocampus and hypothalamus. Other reports using TRPV1 antibodies demonstrate TRPV1 expression in the dentate gyrus, striatum and nucleus accumbens (Musella et al., 2009; Grueter et al., 2010; Chavez et al., 2010; Bhaskaran et al., 2010). These areas are not labeled in the knock in reporter mice, but the discrepancy could be due to a lack of sensitivity in one or both approaches, a phenomenon recently reported for melanopsin expression in the retina (Estevez et al., 2012).

\subsection{TRPV1 Function}

TRPV1 is reported to play roles in learned fear, neuropathic pain and migraine (Caterina \& Julius, 2001; White et al., 2011 for a review). At present, the majority of the work on TRPV1 function focuses on its role in coding noxious stimuli or in the regulation of pain in disease states. TRPV1 also has been proposed to play a role in memory formation via excitatory synapse formation in long-term potentiation (LTP) in the hippocampus (Chavez et al., 2010; Bhaskaran et al., 2010) and neurite pruning in long-term depression (LTD) in the retinocollicular pathway and nucleus accumbens (Maione et al. 2009; Grueter et al., 2010). In both LTP and LTD, TRPV1 increases intracellular calcium concentrations for extended periods of time, resulting in sustained transmitter release from presynaptic terminals.

\subsection{TRPV1 in the retina}


The transient receptor potential vanilloid 1 (TRPV1) is a non-specific cation channel that preferentially passes calcium ions. TRPV1 was first discovered as the receptor for capsaicin, the pungent oil in hot peppers. A few studies have looked at the localization of TRPV1 in the retina using antibodies, but results vary and although it is clear that TRPV1 mRNA and protein are present in the retina the specific cell types that express the TRPV1 remain unknown (Gilliam \& Wensel, 2011). In goldfish, TRPV1-like channels are reported to be expressed in cone terminals (Zimov \& Yazulla 2007). TRPV1 protein expression has been observed in embryonic retinal development (E19 in rat) with the highest levels in the two plexiform layers (Leonelli et al., 2009). Maione and colleagues (2009) report transient expression of TRPV1 at the GC terminals in the superior colliculus during development, which is involved in pruning and refinement of neurites. TRPV1 has been hypothesized to play a role in pressure-induced apoptosis in adult rat GCs (Sappington et al., 2007; Leonelli et al., 2010). ERGs have been recorded to short (5ms) flashes of light in mice that lack TRPV1 protein (TrpV1-/) and results were found to be similar to WT. 


\section{CHAPTER II}

\section{EXPERIMENTAL MATERIALS AND METHODS}

\section{Animals (Chapters III, IV \& V)}

Mouse strains used in electrophysiology experiments to investigate the function of TRPV1 in the retina (chapter III) include: C57BL/6J wild type (WT) mice and age matched B6.129X1-Trpv1<tm1 Jul> $\left(\operatorname{TrpV1}{ }^{-/}\right)$mice (Jackson Laboratory). In order to assess TRPV1 expression (chapter III), a knock in mouse in which Cre was inserted into Exon 15 of TrpV1 (TRPV1 ${ }^{\text {Cre }}$ ) was used. These mice have been used previously to examine expression in brain, DRG and spinal cord (Cavanaugh et al., 2011b; Jackson laboratories). TRPV1 ${ }^{\text {Cre }}$ mice were crossed to B6.Cg-Gt(ROSA)26Sortm9(CAG-tdTomato)Hze/J (Ai9) in which the LoxPSTOP-LoxP TdTomato construct is knocked in at the Gt(ROSA)26Sor locus (Madisen et al., 2010;Jackson laboratories). TRPV1 ${ }^{\text {cre/Ai9 mice express }}$ tdTomato in cells which have ever expressed TRPV1.

Mouse strains used in the investigation of the function and expression of LRIT3 in the retina (chapter V) include the $\mathrm{Nyx}^{\text {nob }}$ and $T r p M 1$ - mice which have been described previously (Cao et al., 2012; Masu et al., 1995; Pardue, et al., 1998; Peachey et al., 2012; Pearring et al., 2011). Trpm1\% (Trpm1 ${ }^{\text {tm1Lex }}$ mice were originally generated by Lexicon Genetics and can be obtained from the European Mouse Mutant Archive (emmanet.org). In thy1-mitoCFP-P (MitoP) 
mice, the thy1-promoter drives expression of CFP to label neuronal mitochondria (Misgeld et al., 2007). In the retinas of these mice, Type 1 OFF BCs are specifically labeled with CFP (Schubert et al., 2008). In VSX1-CFP mice, Type 1 and 2 OFF BCs are fluorescently labeled with CFP (Gift from Rachel Wong). To record from labeled OFF BCs, MitoP or VSX-CFP mice were crossed then backcrossed to Lrit3\% mice that we developed (see 2.1.2 Generation of Lrit3 Zinc Finger Nuclease (ZFN) knockout mice) to obtain MitoP/ Lrit3\% experimental and MitoP/ Lrit3-/+ control mice. For all procedures, mice were anesthetized with a ketamine/xylazine solution (127/12 mg/kg, respectively) diluted in normal mouse ringers. All mice were maintained on a 12:12h light/dark schedule. All experimental procedures were conducted in accordance with regulations described for the ethical care and treatment of animals in the Society for Neuroscience Policies on the Use of Animals in Neuroscience Research and with the approval of the University of Louisville Institutional Animal Care and Use Committee.

1.2 Generation of Lrit3 Zinc Finger Nuclease (ZFN) knockout mice (Chapter V)

$\mathrm{C} 3 \mathrm{H} / \mathrm{HeNTac} / 57 \mathrm{BL} / 6 \mathrm{NTac}$ hybrid embryos (363) were injected with $10 \mathrm{ng} / \mu \mathrm{l}$ Lrit3 ZFN mRNA and 254 viable embryos were implanted into 9 Swiss Webster recipient mothers. Tail biopsies from offspring were collected and genomic DNA isolated using Direct Tail PCR solution (Thermo Scientific) supplemented with 0.2 $\mu \mathrm{g} / \mathrm{ml}$ proteinase $\mathrm{K}$ (Thermo Scientific). Primers (5'CTTTAAACGGAGTCTCGAAGC 3'; 5' CTGACCGCCTCGTTTGGCAC 3') were used to amplify the ZFN targeted region. PCR fragments were either 
sequenced directly or ten fragments were cloned into the TopoBlunt vector (Invitrogen) and then sequenced.

\section{In Vitro Characterization of TRPV1 Expression and Function (Chapter III-IV)}

\subsection{AAV Vector Construction}

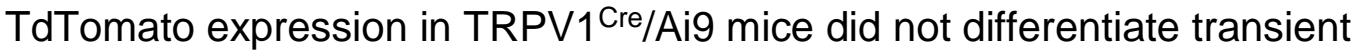
developmental from mature TrpV1 transcription. To assess TRPV1 expression in mature mice, we used adeno-associated virus (AAV, serotype1) to achieve expression of tdTomato in adult retinae. Virus was obtained from the Gene Therapy Program at the University of Pennsylvania. AAV1.CAG.Flex.tdTomato WPRE.bGH (AV-1-ALL864) was used to deliver a Flex-tdTomato construct to retinal cells. Virus was delivered by intravitreal injection $(9.55 \mathrm{e} 12 \mathrm{units} / \mathrm{ml})$ in 1 to 3 month old mice ( $n=9)$ as previously described (Jaubert-Miazza et al., 2005; Su

et al., 2011). Briefly, mice were anesthetized by xylazine/ketamine injection (see 2.3.1 Surgical Preparation). The sclera was pierced with a sharp-tipped glass pipette and excess vitreous was drained. Another pipette, filled with AAV in a sterile PBS and $5 \%$ glycerol solution was inserted into the hole made by the first pipette. The pipette containing the AAV solution was attached to a Picospritzer II (20 psi; Micro Control Instruments, East Sussex, UK) and a 10 msec puff injected 2 to $4 \mu \mathrm{l}$ of solution into the eye. To define TRPV1 expression at the time of sacrifice, mice were euthanized 2 to 4 weeks after viral injection, eyes removed, and retinas dissected for immunohistochemical analysis (see 2.4.2 
Immunohistochemistry). Four out of nine TRPV1 $1^{\text {cre }}$-AAV and 2 TRPV1 ${ }^{\text {cre }} /$ Ai9 mice were transcardially perfused with PBS and $4 \%$ paraformaldehyde, and brains were post-fixed in 4\% paraformaldehyde for 12 hours. Coronal sections $(100 \mu \mathrm{m})$ were obtained using a vibratome (Leica, model\# VT1000S) and mounted in ProLong Gold with DAPI (ThermoFisher Scientific, Cat\# P36931). Retinal projection images were acquired on an Olympus confocal microscope using Fluoview software (see 2.4.3 Confocal Image Acquisition and Morphological Analysis).

\subsection{Immunohistochemistry}

\subsubsection{Tissue preparation}

Retinas were prepared for whole mount and frozen section immunohistochemistry as previously described (Nobles et al., 2012). The eyes were enucleated and the retinas removed from the eyecup. Isolated retinas are fixed in $4 \%$ paraformaldehyde for 15 min followed by three washes $0.1 \mathrm{M}$ PB for 10 min each. Retinas intended for whole mount immunohistochemistry were adhered to nitrocellulose paper (Millipore). Retinas intended for frozen sections were incubated in a series of sucrose solutions of increasing concentration $(5 \%$, $10 \%, 15 \%$ and $20 \%$; Barthel and Raymond,1990) for one hour in each concentration. Retinas were then incubated in OCT:20\% sucrose (2:1; Sakura Finetek) for one hour. Knockout and WT control retinas were stacked together in the same mold and frozen in the OCT:20\% sucrose solution. Twenty-five $\mu \mathrm{m}$ transverse sections were made using a Leica CM1850 Cryostat and mounted to 
slides. Cryo-sections were dried 30 to $45 \mathrm{~min}$ at $37^{\circ} \mathrm{C}$ then incubated in PBS for 10 min prior to immunohistochemistry.

\subsubsection{General Immunohistochemistry}

Wholemount and cryo-sectioned retinas were incubated in $0.5 \%$ Triton X100 in $0.1 \mathrm{M}$ PB (PBX) for $1 \mathrm{~h}$, followed by incubation for $1 \mathrm{~h}$ in a blocking solution of PBX/ $10 \%$ NGS or NDS. Retinas were incubated in primary antibody in blocking solution for 1 day (slices), 2 days (wholemount GCL label) or 7 days (wholemount INL label) at $4^{\circ} \mathrm{C}$ then washed 6 times in PBS for 10 min each wash. Retinas were incubated in the appropriate Alexa conjugated secondary antibody overnight in the dark at $4^{\circ} \mathrm{C}$. A list of the primary and secondary antibodies that were used is in Table 2.1. Retinas were washed and whole retinas were mounted on slides. All slides were covered with Vectashield immuno-mount (Vector Labs, cat\# H-1000). A coverslip was placed over the tissue and sealed with clear nail polish. In some retinas, Choline Acetyl Transferase anti-body (CHAT; 1:1000 anti-goat) was used to label cholinergic ACs in whose processes define the inner and outer sublaminae of the inner plexiform layer for easy identification of OFF and ON processes. After electrophysiological recordings TRPV1 ${ }^{\text {cre }} / \mathrm{Ai9}$ GCs labeled with neurobiotin and Lucifer Yellow (LY) were reacted with streptavidin-Cy2 (1:200, Jackson Immuno cat\# $016-220-084$ ) or antibodies to LY, respectively, to assess their morphology. For all immunohistochemistry experiments looking at the expression of TRPV1, similarly processed dorsal root ganglia which highly express TRPV1 were used 
as positive controls. Tissue processed identically but without primary antibody

was used as negative control.

Table 2.1. Antibodies used to label specific proteins in the retinas of reporter mice. Primary antibody targets include TRPV1and fluorescent proteins to label cells in reporter mice. The appropriate secondary to targets the IgG from the host species in which the primary was raised. Primary Antibodies

\begin{tabular}{|l|l|l|l|}
\hline Antibody target & Host species & Concentration & Company \\
\hline TRPV1 & Guinea Pig & $1: 1000$ & $\begin{array}{l}\text { EMD Millipore, } \\
\text { Darmstadt, } \\
\text { Germany. }\end{array}$ \\
\hline TRPV1 & Guinea Pig & $1: 500$ & $\begin{array}{l}\text { Gift from David } \\
\text { Julius }\end{array}$ \\
\hline TRPV1 & Rabbit & $1: 5000$ & $\begin{array}{l}\text { Alamone Labs, } \\
\text { Jerusalem, Israel }\end{array}$ \\
\hline TRPV1 & Rabbit & $1: 50 \& 1: 500$ & $\begin{array}{l}\text { Neuromics, Edina } \\
\text { MN }\end{array}$ \\
\hline TRPV1 & Goat & $1: 500$ & $\begin{array}{l}\text { Santa Cruz } \\
\text { Biotechnology, } \\
\text { Dallas TX }\end{array}$ \\
\hline ChAT & Goat & $1: 1000$ & $\begin{array}{l}\text { EMD Millipore, } \\
\text { Darmstadt, } \\
\text { Germany. }\end{array}$ \\
\hline RFP & Rabbit & $1: 1000$ & $\begin{array}{l}\text { Invitrogen, Waltham } \\
\text { MA }\end{array}$ \\
\hline GFP & Chicken & $1: 1000$ & $\begin{array}{l}\text { Invitrogen, Waltham } \\
\text { MA }\end{array}$ \\
\hline Melanopsin & Rabbit & $1: 10,000$ & $\begin{array}{l}\text { Gift from Ignacio } \\
\text { Provencio }\end{array}$ \\
\hline Brn3b & Rabbit & $1: 500$ & $\begin{array}{l}\text { Gift from Tudor } \\
\text { Badea }\end{array}$ \\
\hline vGlut3 & Guinea Pig & $1: 5000$ & $\begin{array}{l}\text { Gift from Rebecca } \\
\text { Seal }\end{array}$ \\
\hline GABA & Rabbit & $1: 1000$ & Gift from David Pow \\
\hline Glycine & Rat & $1: 1000$ & Gift from David Pow \\
\hline Lucifer yellow & Rabbit & $1: 1000$ & $\begin{array}{l}\text { Invitrogen, Waltham } \\
\text { MA }\end{array}$ \\
\hline
\end{tabular}

Secondary antibodies

\begin{tabular}{|l|l|l|l|}
\hline Antibody target & Host species & Concentration & Conjugation \\
\hline Rabbit & Goat & $1: 1000$ & Alexa 488 or 546 \\
\hline Guinea Pig & Goat & $1: 1000$ & Alexa 488 \\
\hline Mouse & Goat & $1: 1000$ & Alexa 488 or 546 \\
\hline Rat & Goat & $1: 1000$ & Alexa 546 \\
\hline Chicken & Goat & $1: 1000$ & Alexa 488 or 546 \\
\hline Goat & Donkey & $1: 1000$ & $\begin{array}{l}\text { Alexa } 488 \text { or } 546 \\
\text { or 647 }\end{array}$ \\
\hline Rabbit & Donkey & $1: 1000$ & Alexa 488 or 647 \\
\hline
\end{tabular}




\subsubsection{Tyramide Signal Amplification}

In order to detect the weak melanopsin or TRPV1 expression, tyramide signal amplification was used (TSA kit \#12; Invitrogen) as reported by Estevez and colleagues (2012). Briefly, retinas were incubated in $1 \% \mathrm{H}_{2} \mathrm{O}_{2}$ in $0.5 \%$ PBX for $1 \mathrm{~h}$ to quench peroxidase activity. Retinas were then washed (always in PBS 6 times for $10 \mathrm{~min}$ ) and incubated in TSA blocking solution for $2 \mathrm{~h}$ and then melanopsin antibody or rabbit anti-TRPV1 in TSA blocking solution for 2 days at $4^{\circ} \mathrm{C}$. Tissue was washed then incubated in TSA blocking solution for $1 \mathrm{~h}$, followed by goat anti-rabbit HRP secondary (1:100) in TSA blocking solution for $2 \mathrm{~h}$. Retinas were again washed then incubated in tyramide-488 (1:100) in 1_Plus Amplification Diluent (Perkin Elmer) for exactly 5 min then washed and mounted on a glass slide with Vectashield.

\subsubsection{Zenon Conjugation of Melanopsin Antibody}

Antibody to melanopsin and the transcription factor Brn3b were used to assess the co-expression in the TRPV1 expressing intrinsically photosensitive retinal ganglion cells (ipGCs; Berson et al., 2010) in wholemount retinas.

Because both primary antibodies were raised in rabbit, melanopsin antibody was first conjugated to Alexa 647 using a Zenon labeling kit (ThermoFisher Scientific, cat\# Z25308). One $\mu \mathrm{L}$ melanopsin antibody and $5 \mu \mathrm{L}$ Alexa 647 conjugate were incubated together at room temperature for 5 min then incubated with an additional $5 \mu \mathrm{L}$ of Zenon blocking reagent. The conjugated antibody was applied after the completion of the Brn3b primary and secondary antibody incubation 
(see 2.2.2. General Immunohistochemistry). The retina was then incubated in blocking solution for $1 \mathrm{~h}$ and conjugated melanopsin (1:50) in blocking solution for $2 \mathrm{~h}$. The retina was washed then post-fixed in $4 \%$ paraformaldehyde for $15 \mathrm{~min}$, washed 3 times 5 min, mounted on a slide and imaged immediately. The Alexa 647 fluorescence faded significantly the next day.

\subsection{Confocal Image Acquisition and Morphological Analysis}

TdTomato expression was examined in TRPV1 ${ }^{\text {Cre }} /$ Ai9 and TRPV1 ${ }^{\text {cre }}$-AAV retinal whole mount and transverse retinal sections using confocal microscopy as previously reported (Zhang et al., 2014). Images were acquired on an Olympus FV1000 confocal microscope. Z-stack images of tdTomato positive cells and Lucifer yellow and neurobiotin-streptavidin labeled GCs were acquired with 0.4 $\mu \mathrm{m} z$-step using a $40 \times$ water immersion lens, NA 1.15. Images of retinal slices were collected using $60 \times$ oil-immersion lens, NA 1.4. High resolution scanning was performed at $1024 \times 1024$ pixels and images were analyzed using the Olympus Fluoview software (FV10-ASW, Olympus Corporation). Dendritic area was the area of a polygon connecting the tips of the dendrites. Soma area was the area of a smooth polygon outlining the widest extent of the soma. Percent stratification in the IPL was measured using Image $\mathrm{J}$ software on individual $90^{\circ}$ rotations of the z-stack image. The INL and GCL were $0 \%$ and $100 \%$ respectively. Percent stratification of CHAT bands was measured as reference. All data were saved and analyzed using Microsoft Excel and Graphpad Prism statistical software. Each cell was classified according to the GC (Sun et al., 2002; Berson et al., 2010), displaced amacrine cell (dAC; Pérez De Sevilla Müller 
et al., 2007; Zhu et al., 2014) or AC (MacNeil et al., 1999; Haverkamp et al., 2004; Lin and Masland 2006 \& Kay et al., 2011) that was morphologically similar.

The densities and distributions of each cell type were calculated.

Wholemount images $(500 \mu \mathrm{m} \times 500 \mu \mathrm{m})$ were collected from the dorsal, nasal, ventral and temporal quadrants from three TRPV1 1 cre/Ai9 retinas which had been labeled with melanopsin antibody using the TSA method. GCs which did and did not co-express td-tomato and melanopsin were counted in each section to establish the densities of each cell type. Brightly labeled GCs which were brightly labeled with melanopsin were distinguished from dimly labeled and regarded as separate classes (Berson et al., 2010). The coverage factor for each GC class was computed by multiplying the mean dendritic area of each class by the density. GC classes with coverage factors less $<1$ have space between cells and >1 have overlapping dendrites (Wässle, 2004).

\subsection{Whole-cell patch clamp recordings}

\subsubsection{Tissue preparation}

Whole cell patch clamp recordings from GCs was performed as previously described (Ray et al., 2014; Zhang et al., 2014). Eyes were removed and retinas were prepared and dissected under dim red light for GC recordings in Ringer's solution (in mM, $110 \mathrm{NaCl}, 2.5 \mathrm{KCl}, 1 \mathrm{CaCl}_{2}, 1.6 \mathrm{MgCl}_{2}, 10 \mathrm{D}$-glucose and 22 $\mathrm{NaHCO}_{3}$, bubbled with $5 \% \mathrm{CO}_{2} / 95 \% \mathrm{O}_{2}, \mathrm{pH}$ 7.4). Retinas were quartered and each quarter was mounted on a cover slip and placed in a recording chamber. During the experiment, the retinas were superfused with oxygenated extracellular 
solution at $34-35^{\circ} \mathrm{C}$. Recording electrodes were pulled from borosilicate glass (FHC) on a P-97 Flaming/Brown Micropipette Puller (Sutter Instruments).

Electrode resistance measured between with 5-8 M 2 . The glass pipettes were back-filled with (in mM) $112.5 \mathrm{CsCH}_{3} \mathrm{SO}_{3}, 1 \mathrm{MgSO}_{4}, 7.8 \times 10^{-3} \mathrm{CaCl}_{2}, 0.5$ BAPTA, 10 HEPES, 4 ATP-Na2, 0.5 GTP-Na3, and 5 lidocaine $N$-ethyl bromide (QX314$\mathrm{Br}$ ). To visualize GCs for morphological classification, the intracellular solution contained 7.5 neurobiotin chloride ( $\mathrm{pH} 7.2$ adjusted with $\mathrm{CsOH}$ ) and 2.2 Lucifer Yellow CH Lithium Salt.

\subsubsection{Electrophysiology and Pharmacology}

TRPV1 1 cre/Ai9 GCs were visualized using brief exposure to green light. A $\mathrm{G} \Omega$ seal was created on the soma and only GCs with series resistance $<25 \mathrm{M} \Omega$ were used for recording. All voltages were corrected for the measured liquid junction potential of $17 \mathrm{mV}$. Whole cell GC current were recorded using an Axon Multiclamp 700B amplifier (Molecular Devices) and signals were digitized at 10 kHz with a Digidata 1440A (MDS Analytical Technologies). Signals were filtered at $2.4 \mathrm{kHz}$ with a four-pole Bessel low-pass filter. Clampex 10.2 software (MDS Analytical Technologies) was used to generate command outputs and acquire analog whole-cell current. Off-line, a $20 \mathrm{~Hz}$ eight-pole Bessel low-pass filter was applied to the data and analyses of data were performed using Clampfit 10.4. To block excitatory inputs, bath solution was supplemented with L-AP4 $(4 \mu \mathrm{M})$ to saturate mGluR6 receptors as well as DAP-5 $(50 \mu \mathrm{M})$ and CNQX $(20 \mu \mathrm{M})$, to block NMDA and non-NMDA receptors, respectively. All reagents were purchased from Tocris Bioscience. Capsaicin evoked currents were recorded by 
applying capsaicin $(30 \mu \mathrm{M})$ in extracellular solution with blockers to the bath for 35 min while holding the GC membrane potential at $-60 \mathrm{mV}$. Consistent with previous calcium imaging studies in dissociated GCs (Ryskamp et al., 2014), current responses to bath application of capsaicin were slow, reaching peak amplitude within 2 to 3 min after response onset and lasting several minutes. We found that GC membrane currents fluctuated in the absence of capsaicin with similar timing as capsaicin evoked responses. To differentiate capsaicin evoked responses from normal fluctuations, control experiments were performed. GC current responses were recorded to Ringer's solution with blockers applied from a second bath solution.

Capsaicin evoked currents were also recorded from two ACs in whole mount retinas in the Borghuis lab as previously described (Borghuis et al., 2011). The recording protocol and reagents were similar, but differences existed in the equipment. The main differences from the protocol above are as follows:

1. Retinas were dissected under infrared illumination and kept whole rather than quartered.

2. Retinas were bathed in oxygenated Ames (Sigma-Aldrich) instead of Ringers.

3. Fluorescent ACs were imaged using a two-photon microscope rather than epifluorescence.

4. ACs were filled with Alexa 488 dye (Invitrogen) for visualization. Morphological classification was successful in one AC.

All differences are unlikely to alter the results.

\subsubsection{Whole cell patch clamp analysis}


Baseline current was measured as the average over two minutes before response onset. The peak amplitude of the slow current was measured as the minimum from baseline. A capsaicin response was defined as larger amplitude fluctuation than control. All cells with capsaicin evoked responses had similar times to onset.

\section{In Vitro Electrophysiology Recordings from Retinal Ganglion Cells using Multi-electrode Array (Chapter IV \& V)}

\subsection{In vitro MEA recordings}

The procedures for multi-electrode array (MEA) recordings have been previously published (Fransen et al., 2015). Briefly, mice were dark adapted overnight and their retinas were dissected under dim red illumination to preserve the photoreceptor responses. Chemical vitrectomy was performed using proteases collagenase $(12 \mathrm{U} / \mathrm{mL})$ \& hyaluronidase $(37.13 \mathrm{uM})$ in Ringers applied for $15 \mathrm{~min}$ then washed three times to remove proteases. The dissected retina was quartered with anatomical orientation preserved. Retinal pieces were incubated in bicarbonate buffered Ringer's solution (see "Whole cell patch clamp recordings") within a light protected oxygenated chamber. Oxygenated Ringers was continuously pumped through the temperature controlled $\left(37^{\circ} \mathrm{C}\right)$ recording chamber (Multichannel systems). To record the responses of GCs, a quarter of the retina was placed in the recording chamber and held in place with GCs next to the electrodes on a 60 electrode array with a transparent cell filter membrane 
(Fisher Scientific) held down by a platinum ring. Retinal piece in the recording chamber were maintained in darkness for $45 \mathrm{~min}$ to $1 \mathrm{~h}$ prior to experiments. Spiking activity was bandpass filtered $(3000-100 \mathrm{~Hz}$; MC Rack software, Multichannel systems)

\subsection{Visual Stimuli}

We established a threshold of response to dim light and assessed the spatial and temporal properties of GCs in photopic conditions. Five full-field light increments of 5 second duration were presented with increasing intensity. Responses were recorded to $0.6,1.4 \& 3.1 \log \mathrm{R}^{\star} / \mathrm{rod} / \mathrm{sec}$ stimuli against a dark background. The retina was then adapted to a $2.6 \log \mathrm{R}^{\star} / \mathrm{rod} / \mathrm{sec}$ background for $5 \mathrm{~min}$ before recording responses to $4.1 \& 5.4 \log \mathrm{R}^{\star} / \mathrm{rod} / \mathrm{sec}$ stimuli against the same background. Stimuli were projected by a mini-projector (HP Notebook Consumer Projection Companion model\# AX325AA) onto the retina through the microscope optics. Recordings in WT retinae showed that under our dark adaptation conditions, $0.6 \log \mathrm{R}^{\star} / \mathrm{rod} / \mathrm{sec}$ stimulus was the minimum intensity that evoked a response in a minority of GCs (18\%). Roughly half of WT GCs responded at $1.4 \log R^{\star} / \mathrm{rod} / \mathrm{sec}$, and almost all responded to $3.1 \log R^{\star} / \mathrm{rod} / \mathrm{sec}$

(Fig. 3.1a). Under light adapted conditions, $4.1 \log \mathrm{R}^{\star} / \mathrm{rod} / \mathrm{sec}$ evoked responses in the largest \% of GCs and $5.4 \log R^{*} / \mathrm{rod} / \mathrm{sec}$ evoked the largest \% of ON/OFF responses (Fig. 3.1a \& d, respectively).

\subsection{Analysis of MEA Data}


Analysis of the MEA recordings also have been described previously (Fransen et al., 2015). Spikes were sorted according to their principle components and clustered according to similarities in spike amplitude and time stamp, keeping only cells which were isolated from the noise (Offline spike sorter version 3, Plexon, Inc.). To verify that each cluster contained spikes from a single cell, only spikes with an interspike interval greater than $1 \mathrm{msec}$ were included in the same cluster. Each cell was analyzed according to the stimulus presented. To analyze full-field stimuli, average PSTHs and raster plots of each cell for each stimulus luminance value were measured and compared, similar to the analyses described by Nobles and colleagues (2012). Briefly, GCs were classified according to their response to the light onset (ON), offset (OFF), or both (ON/OFF). Responses were further classified according to the duration of their response. Sustained cells were defined as those whose excitatory response remained 3 SEM above mean spontaneous level for the entire duration of stimulus presentation ( $5 \mathrm{~s}$ ). Transient GCs had excitatory responses that returned to the mean spontaneous level within the first $2 \mathrm{~s}$. A small population of GCs responded to the offset of light for $2>5 \mathrm{~s}$ and was analyzed separately (Fig. $3.3 c \& d)$.

\section{In Vivo Electrophysiology Recordings from Optic Nerve Fibers (Chapter IV)}

\subsection{Surgical Preparation}

Surgical procedures were performed at light adapted levels and have 
been previously described (Nobles et al., 2012; Sagdullaev and McCall, 2005). Briefly, anesthesia was induced with an intraperitoneal injection of a Ringer's solution containing ketamine $(127 \mathrm{mg} / \mathrm{kg})$ and xylazine $(12 \mathrm{mg} / \mathrm{kg})$. Anesthesia was maintained throughout the experiments with supplemental subcutaneous injections (50\% of initial concentration) administered about every 45 min. The head was secured in a stereotaxic frame (David Kopf Instruments) with ear cups and a bite bar. Body temperature was maintained at $37^{\circ} \mathrm{C}$ with a feedback controlled heating pad (TC-1000; CWE). Eyes were dilated with topical tropicamide $(0.25 \%)$ and phenylephrine hydrochloride $(0.6 \%)$. Clear zeropowered lenses (Sagdullaev et al, 2004) moistened with artificial tears (Akwa Tears, Akorn, Inc.) were placed over the eyes to prevent drying of the corneas. A craniotomy was performed anterior to the Bregma suture and the overlying cortex was removed using suction to expose the optic nerve.

\subsection{Electrophysiology Recordings from Optic Nerve Fibers}

Tungsten rods were etched to form electrodes and coated in acrylic (impedance 50-100 K $\Omega$ ). A reference electrode was inserted subcutaneously. Action potentials were recorded from individual axons and amplified (Xcell3 microelectrode amplifier, FHC). Isolated spikes were displayed on an oscilloscope (60MHz, Tektronix Inc.) and played on an audio monitor (AM7, Grass Instruments) for direct feedback between the stimulus and response. Spikes rising through a level designated above noise were digitized (Power 1402, CED) and recorded for offline analysis (Spike 2, CED). Spikes were accumulated within a $50 \mathrm{~ms}$ bin width and displayed as post-stimulus time histograms 
(PSTHs). Each average PSTH was smoothed by fitting it with a raised cosine function with a $50 \mathrm{~ms}$ smoothing interval to minimize alteration of the peak firing rate and maximize signal-to-noise ratio (Sagdullaev and McCall, 2005).

\subsection{Quantitative Characterization of GC Visual Response Properties}

The position, spatial extent and RF center sign of each GC were determined first using a handheld light source projected onto a screen located 25 $\mathrm{cm}$ from the anterior nodal point of the eye. All quantitative measures of GC visual responses and receptive field properties were evoked by stimuli (VisionWorks; Vision Research) presented on a CRT display monitor with a mean luminance of $20 \mathrm{~cd} / \mathrm{m}^{2}$ (Eizo E120 FlexScan FXC7). All stimulus and RF dimensions are presented in degrees of visual angle (corrected for monitor distance). Responses were recorded to a series of $67 \%$ contrast spot increments or decrements from background $\left(20 \mathrm{~cd} / \mathrm{m}^{2}\right)$ corresponding to the preferred stimulus of ON or OFF GCs respectively. To characterize the RF organization, spots were centered on the mapped RF whose outer diameter ranges from 2 to $25 \mathrm{~cm}$ or 4.6 to $52.7^{\circ}$ visual angle. Duration of the spot presentation was $5 \mathrm{sec}$, with a $6 \mathrm{sec}$ interstimulus interval. Each spot diameter was presented 8 times and interleaved randomly along with 8 blank trials in which only background was presented. To estimate the contribution of RF surround, responses were recorded to $25 \mathrm{~cm}$ diameter, $67 \%$ contrast spot increments or decrements from background $\left(20 \mathrm{~cd} / \mathrm{m}^{2}\right)$ corresponding to the preferred stimulus of ON or OFF GCs respectively. A series of background luminance $\left(20 \mathrm{~cd} / \mathrm{m}^{2}\right)$ inner spots whose outer diameter ranges from 2 to $16.5 \mathrm{~cm}$ was presented. The inner and 
outer spot arrangement forms an annulus. Duration of the annulus presentation was $5 \mathrm{sec}$, with a $6 \mathrm{sec}$ interstimulus interval. Each annulus of inner spot diameter was presented 8 times and interleaved randomly along with and 8 blank trials in which only background was presented.

\subsection{Analysis of responses from intact preparation}

The spike trains were displayed as a peri-stimulus time histogram in which the numbers of spikes per second are graphed over time. Responses to each spot diameter or luminance were averaged and smoothed to maximize signal to noise ratio. The baseline firing rate, peak firing rate, mean firing rate during stimulation and response duration were calculated from each smoothed function and compared between genotypes. The peak response amplitude and response duration were graphed as a function of spot diameter to produce an area response function (ARF). In WT GCs the peak response has an arch shape because peak response will increase as the spot size increases until the spot equals the functional RF size of the cell. As the spot diameter increases beyond the RF center, the surround suppression decreases the peak response amplitude. The peak of the area response function was considered the optimal

spot size of the GC. The duration of the area response function is typically flat in WT GCs because durations are consistent regardless of spot size. Peak amplitude, response duration and mean response rate of the optimal spot size were compared between genotypes. 


\section{Characterization of LRIT3 Expression and Function (Chapter V)}

\subsection{Immunohistochemistry for LRIT3}

Table 2.2. Antibodies used to label specific proteins in the retinas of $\operatorname{TrpM1}{ }^{-1}, \mathrm{NyX}^{\mathrm{nob}}, \mathrm{Lrit}^{-1+}$ and Lrit3 ${ }^{-}$mice. Primary antibodies were used to fluorescently label proteins that constitute the mGluR6-TRPM1 signalplex in dendritic tips of ON BCs. Antibodies to Cone Arrestin and the lectin PNA were used to label proteins in the cone terminals. Antibodies to Cacna1f labeled the protein in the rod and cone terminals. Pikachurin is an extracellular protein in the synapse between photoreceptors and ON BCs.

Primary Antibodies

\begin{tabular}{|l|l|l|l|}
\hline Antibody target & Host species & Concentration & Source \\
\hline mGluR6 & Goat & $1: 2000$ & $\begin{array}{l}\text { Gift from Catherine } \\
\text { Morgans }\end{array}$ \\
\hline TRPM1 & Goat & $1: 2000$ & $\begin{array}{l}\text { Gift from Kirill } \\
\text { Martenmyanov }\end{array}$ \\
\hline GPR179 & Sheep & $1: 2000$ & $\begin{array}{l}\text { Gift from Catherine } \\
\text { Morgans }\end{array}$ \\
\hline RGS7 & Sheep & $1: 1000$ & $\begin{array}{l}\text { Gift from Catherine } \\
\text { Morgans }\end{array}$ \\
\hline RGS11 & Sheep & $1: 1000$ & $\begin{array}{l}\text { Gift from Catherine } \\
\text { Morgans }\end{array}$ \\
\hline R9AP & Sheep & $1: 2000$ & $\begin{array}{l}\text { Gift from Catherine } \\
\text { Morgans }\end{array}$ \\
\hline G35 & Goat & $1: 1000$ & $\begin{array}{l}\text { Gift from Catherine } \\
\text { Morgans }\end{array}$ \\
\hline PNA ${ }^{*}$ & $\begin{array}{l}\text { Mouse (568 } \\
\text { conjugated })\end{array}$ & $1: 1000$ & $\begin{array}{l}\text { ThermoFisher; cat \# } \\
\text { L32458 }\end{array}$ \\
\hline Cone Arrestin & Rabbit & $1: 1000$ & Gift from Cheryl Craft \\
\hline Pikachurin & Rabbit & $1: 1000$ & $\begin{array}{l}\text { Gift from Catherine } \\
\text { Morgans }\end{array}$ \\
\hline PSD95 & Mouse & $1: 1000$ & $\begin{array}{l}\text { Gift from Catherine } \\
\text { Morgans }\end{array}$ \\
\hline Cacna1f & Sheep & $\begin{array}{l}\text { Gift from Catherine } \\
\text { Morgans }\end{array}$ \\
\hline *PNA is not a protein but carbohydrate moiety to which it binds. & \\
\hline
\end{tabular}

Immunohistochemistry was performed as described above $(2.2$

Immunohistochemistry). Table 2.2 is a list of the primary antibodies used in Chapter V.

\subsection{Electroretinography}


Mice were dark adapted overnight and anesthetized (see Animals). The anesthetized mouse was prepared for ERG recordings under dim red light. Pupils were dilated and accommodation relaxed with topical applications of $0.625 \%$ phenylephrine hydrochloride and $0.25 \%$ Tropicamide and corneal surface anesthetized using 1\% Proparacaine HCL. Body temperature was maintained via an electric heating pad (TC1000 Temperature control, CWE Inc.). A clear acrylic contact lens with a gold electrode (LKC technologies Inc.) was placed on the cornea and wet with artificial tears (Tears Again, OCuSOFT, Inc.). Ground and reference needle electrodes were placed in the tail and on the midline of the forehead, respectively. A 20 min dark-adaptation preceded the presentation of full field strobe flashes presented in a Ganzfeld (LKC UTAS Visual Diagnostics System with Big Shot Ganzfeld). For scotopic responses flashes were presented on a dark background and for photopic responses there was a 5 min light adaptation period and flashes were presented on a rod saturating background $\left(20 \mathrm{~cd} / \mathrm{m}^{2}\right)$. The luminance of the flashes ranged from -3.6 to $1.4 \log \mathrm{cd} \mathrm{sec} / \mathrm{m}^{2}$.

\subsection{Retinal slice preparation and $B C$ whole cell patch clamp recording}

Anesthetized mice were euthanized by cervical dislocation, eyes were enucleated and retinas were removed and placed in Ames solution (SigmaAldrich). Retinas were placed on nitrocellulose paper (Millipore) and sliced perpendicular to the retinal layers using a tissue slicer. The retinal slices were then adhered in place in the recording chamber using vacuum grease. Recording electrodes were pulled on a P-97 Flaming/Brown Micropipette Puller (Sutter Instruments Co., Novato, CA) from borosilicate glass (FHC, Inc., Bowdoin, ME). 
Electrode resistance measured between 6-9 M . Electrodes were filled with Csgluconate intracellular solution $(20 \mathrm{mM} \mathrm{CsCl}, 107 \mathrm{mM} \mathrm{CsOH}, 107 \mathrm{mM} \mathrm{D-}$ Gluconic Acid, 10 mM NaHEPES, 10 mM BAPTA, 4 mM ATP, and 1 mM GTP). $1 \%$ sulforhodamine was included in the intracellular solution to visualize the cell and classify the morphology (Ghosh, et al., 2004).

Retinal slices were bathed in Ames solution supplemented with the following inhibitory blockers: $1 \mu \mathrm{M}$ strychnine, $100 \mu \mathrm{M}$ picrotoxin and $50 \mu \mathrm{M} 6$ tetrahydropyridin-4-yl methylphosphinic acid (TPMPA). L-AP4 $(4 \mu \mathrm{M})$ also was added to the bath solution to saturate mGluR6 receptors. Rod ON BC somas were targeted for whole cell patch clamp recording. A 2-4 G $\Omega$ seal was created on the cell body. Cells with an input resistance $\sim 1 \mathrm{G} \Omega$ and access resistance < $25 \mathrm{M} \Omega$ were used for recording. The recording chamber was maintained at 34$35^{\circ} \mathrm{C}$. A Picospritzer II (Parker Instrumentation, Cleveland, $\mathrm{OH}$ ) was used to pressure apply pharmacological agents onto rod ON BC dendritic tips in the outer plexiform layer (OPL). The agents used were the mGluR6 receptor antagonist $\alpha$ cyclopropyl-4-phosphonophenylglycine (CPPG $0.6 \mathrm{mM}$ or $3 \mathrm{mM}$ ) or the TRPM1 agonist capsaicin $(10 \mu \mathrm{M})$. All reagents were purchased from Sigma-Aldrich, except for L-AP4 and capsaicin, which were purchased from Tocris Bioscience (Avonmouth, Bristol, BS11 9QD United Kingdom).

\subsection{Voltage Clamp Protocols}

Voltage clamp responses were recorded using a Multiclamp 700A amplifier with a Digidata 1440A digitizer (MDS Analytical Technologies, Union City, CA) and filtered at $2.4 \mathrm{kHz}$ with a four-pole Bessel low pass filter, sampled 
at $10 \mathrm{kHz}$. Clampex 10.2 software (MDS Analytical Technologies, Sunnyvale, CA) was used to generate command outputs and acquire and analyze analog whole cell current. Rod ON BCs were voltage clamped at +50mV (Nawy, 2004; Shen et al., 2009). Capsaicin was puffed at the dendrites for either $200 \mathrm{msec}$ or 1 sec. Three to five responses were recorded from RBC and then averaged. The first $1.5 \mathrm{sec}$ of each rod $\mathrm{ON} \mathrm{BC}$ recording was used to measure variance and standing current. Clampfit 10.2 was used for offline analyses of data. Currents were filtered offline with an 8-pole Bessel low pass filter at $20 \mathrm{~Hz}$. Prism 5.04 software (Graphpad Software, Inc., La Jolla, CA) was used to perform the following statistical analyses as suited for the necessary comparison: two-way repeated measures ANOVAs, two-way ANOVAs, one-way ANOVAs, or t-tests. Statistical significance $=P<0.05$.

\subsection{MEA recordings}

Light evoked spiking responses to 2 s full-field stimulus $\left(5.4 \log \mathrm{R}^{\star} / \mathrm{rod} / \mathrm{sec}\right.$, 20 trials, 10s ISI) were recorded from Lrit3 $^{-/ 4}$, Lrit3 ${ }^{-/}$and $m$ GluR6 $6^{-/}$retinas using MEA as described above (see 3.1 In vitro MEA recordings). After obtaining baseline light responses, L-AP4 $(4 \mu \mathrm{M})$ or ACET $(20 \mu \mathrm{M})$ was added to the bath solution to saturate mGluR6 receptors or block Kainate receptors respectively. Light responses were recorded in the presence of reagents and 20 min after Ringer's wash. Data was analyzed as described above (see 3.3 Analysis of MEA).

\section{Statistical Analysis (Chapters III, IV \& V)}


Statistical analyses were performed using Prism5 Software (GraphPad). Distributions of each parameter were tested for normality using the D'Agostino and Pearson omnibus test and appropriate parametric or non-parametric statistical analyses were used. For each morphological and electrophysiological measure, t-test with Welch's correction was used for two sample comparison. One-way ANOVA with Bonferroni's post-hoc tests were used for comparisons with three or more normally distributed samples. Kruskal Wallace with Dunns post-hoc tests were used for non-parametric comparisons. Two-way ANOVA with Bonferroni's post-hoc tests was used to compare response components across spot size or luminance. In all figures, data are plotted as the mean \pm SEM and significance is indicated as ${ }^{*} p<0.05,{ }^{* *} p<0.01,{ }^{* * *} p<0.001,{ }^{* * *} p<0.0001$. For MEA recordings, alpha $\leq 0.01$ was considered significant (see Appendix I). For all other comparisons alpha $\leq 0.05$ was considered significant. 


\section{CHAPTER III}

\section{TRPV1 MODULATES RETINAL GANGLION CELL SPIKING ACTIVITY IN THE MOUSE}

\section{Introduction}

The transient receptor potential vanilloid-1 (TRPV1) channel is a nonselective cation channel with an affinity for $\mathrm{Ca}^{2+}$. TRPV1 has been extensively studied in A $\bar{\delta}$ and $\mathrm{C}$-fiber nociceptors in the PNS. There, the channel can be activated by many ligands including capsaicin, low pH, endovanilloids such as anandamide or by stimulation with noxious heat or pressure (Caterina \& Julius, 2001; Moreira et al., 2012). Activation of TRPV1 in the peripheral and central terminals of primary nociceptors results in the robust influx of $\mathrm{Ca}^{2+}$ and subsequent prolonged release of glutamate to post-synaptic spinal cord neurons. TRPV1 also enhances the sustained release of $\mathrm{Ca}^{2+}$ from intracellular stores, resulting in sensitization of the nociceptors long after the initial stimulus (Medvedeva et al., 2009). In this way, TRPV1 activity in the PNS plays a role in nociception, inflammation, chronic pain, nociceptor sensitization and desensitization, and apoptosis (Caterina et al., 2000; Caterina \& Julius 2001; Medvedeva et al., 2009). 
The literature regarding the expression and function of TRPV1 in the CNS is contradictory. Cavanaugh and colleagues (2011b), using reporter mice, determined TRPV1 expression in the brain is confined to a few discrete areas, most notably in the periaquaductal gray, Cajal-Rhetsius cells of the hippocampus, and hypothalamus in reporter mice. Other studies demonstrate TRPV1expression and function in the hippocampal pyramidal cells (Gibson et al., 2008), dentate gyrus (Chavez et al., 2010), striatum (Musella et al., 2009), nucleus accumbens (Grueter et al., 2010; Bhaskaran et al., 2010; Renteria et al., 2014) and superior colliculus (Maione et al., 2011). TRPV1 also is expressed in the retina (Gilliam \& Wensel, 2011; Sappington et al., 2009 \& 2015; Leonelli et al., 2009). TRPV1 in the CNS is reported to play roles in thermoregulation, learned fear, neuropathic pain, and migraine (Caterina \& Julius, 2001; White et al., 2011 for review). TRPV1 also has been proposed to play a role in memory formation by regulation of long-term potentiation (LTP) and excitatory synapse formation in the hippocampus (Chavez et al., 2010; Bhaskaran et al., 2010). TRPV1 reportedly regulates long-term depression (LTD) and subsequent neurite pruning in the retino-collicular pathway and nucleus accumbens (Maione et al. 2009; Grueter et al., 2010). In the CNS, TRPV1 is likely gated by endovanilloids such as anandamide (Gibson et al., 2008; Maione et al., 2011; Kawahara et al., 2011; Ryskamp et al., 2014). In both LTP and LTD, TRPV1 activation by anandamide results in $\mathrm{Ca}^{2+}$ influx, prolonged increases in intracellular $\left[\mathrm{Ca}^{2+}\right]$, and subsequent sustained neurotransmitter release from presynaptic terminals. TRPV1 activity in the brain has been implicated in diseases such as fibromyalgia 
and other chronic pain disorders, Parkinson's disease, Huntington's disease, anxiety and depression. Given roles assigned to TRPV1 in the CNS, clarifying its specific function in neural processing is valuable.

TRPV1in the retina has been implicated in disorders such as glaucoma and ischemia induced apoptosis, but the role of TRPV1 in visual signaling has not been investigated. Previous publications report TRPV1 protein expression in the inner retina of rodents (Sappington et al., 2009 \& Leonelli et al., 2009 \& 2013), goldfish (Zimov \&Yazulla, 2007), and non-human primates (Sappington et al., 2015). Similar to the role of TRPV1 in the PNS, the protein is thought to regulate $\mathrm{Ca}^{2+}$ in the ganglion cell layer $(\mathrm{GCL})$ and inner nuclear layer $(\mathrm{INL})$ of the retina (Sappington et al., 2009 \& 2015; Leonelli et al., 2010; Ryskamp et al., 2014). In calcium imaging studies, capsaicin, a known agonist of TRPV1, as well as TRPM1, (Shen et al., 2009) increases intracellular $\left[\mathrm{Ca}^{2+}\right]$ in dissociated ganglion cells (GCs; Sappington et al., 2011; Ryskamp et al., 2014). Prolonged capsaicin induced increases in intracellular $\left[\mathrm{Ca}^{2+}\right]$ promotes apoptosis in the inner retina (Leonelli et al, 2011; Sappington et al., 2011). Capsazepine, a known antagonist of TRPV1 and TRPM1 alters neuronal and synaptic development in the rat inner retina (Leonelli et al., 2011). In mouse models of glaucoma, capsazepine blocked pressure induced intracellular $\left[\mathrm{Ca}^{2+}\right]$ increases in dissociated GCs (Sappington et al., 2011) and may be neuroprotective. However selective elimination of TRPV1 protein using knockout mice leads to increased GC death compared to wild type in glaucoma models (Sappington et al., 2015). This suggests TRPV1 activity is neuroprotective in models of retinal dysfunction. 
The role of TRPV1 in normal retinal function has not been studied. To this end, I made use of the parallel visual pathways within the retina to investigate the role of TRPV1 function in normal visual signaling.

To investigate the role of TRPV1 in normal vision, I compared visual responses in WT and TrpV1\% GCs. One of the parallel pathways is established by the input from rods and cones, which differentially signal at low and high luminance levels, respectively. Within the rod pathway, there are multiple luminance dependent signaling pathways, which transfer visual information from the rods to GCs (Bloomfield \& Völgyi, 2009). At visual threshold, rod signaling arises through a primary pathway to rod bipolar cells (RBCs) and through their synapses on All amacrine cells (ACs; Völgyi et al., 2004; see Bloomfield and Dacheux, 2001 for review). All ACs transfer information through gap junctions with $\mathrm{ON}$ cone BCs (CBCs), which stratify in sublaminae (s) three to five in the IPL. All ACs form inhibitory glycinergic synaptic connections with OFF CBCs which stratify in s 1-2 (Kolb \& Nelson, 1983; Strettoi et al., 1992). Völgyi and colleagues (2004) report a proportion of OFF and ON GCs which function as the most sensitive light detectors and receive rod signals only through this primary pathway.

A feedback inhibitory circuit at the RBC to the All synapse further enhances signaling at visual threshold. The All and GABAergic A17 form a dyad synapse with the RBC (Kolb \& Famiglietti, 1974; Famiglietti \& Kolb, 1975; Strettoi et al., 1990; Strettoi et al., 1992; Chun et al., 1993; Kim et al., 1998). The A17 receives glutamatergic input from the $\mathrm{RBC}$ in response to dim light. The $\mathrm{A} 17$ then 
inhibits the RBC though feedback GABA release onto $G A B A_{A}$ and $G A B A_{c}$ receptors in the RBC terminal (Eggers \& Lukasiewicz, 2011). GABA $A$ and $G A B A_{c}$ receptors differ in their kinetic properties. GABA $A$ rapidly turns on and off within several milliseconds (Eggers and Lukasiewicz, 2006). In contrast, GABAc responds more slowly and the duration of the response lasts tens to hundreds of milliseconds (Chang \& Weiss, 1999; McCall et al., 2002). Tonic A17-mediated GABA release in the dark likely decreases spontaneous glutamate release from RBCs, a process that enhances the signal to noise ratio to dim light at the visual threshold (Grimes et al., 2015).

As luminance increases a secondary rod pathway transfers the visual signal to cones via gap junctions (Bloomfield and Völgyi, 2009). This gap junction coupling increased the visual information being transferred from the cones to the CBCs to enhance the sensitivity of the cone pathway to light. However, the increased spread of signal results in low visual acuity. In daylight conditions, gap junction coupling in the retina decreases prevents response saturation and increases visual acuity (Witkovsky, 2004). Rods transfer visual signal synaptically to OFF CBCs in the tertiary rod pathway at higher luminance values.

In the cone pathway, when the rod signal is saturated, visual information is transferred from cones to OFF and ON CBCs, forming the ON and OFF parallel pathways (see Wässle et al., 2004 for review). Light decrements result in increased glutamate release from cones to OFF and ON CBCs, causing OFF CBC to depolarize and ON CBCs, which have a sign inverting synapse (see Chapter 1, Fig. 1.3), to hyperpolarize. Light increments result in decreased 
glutamate release from cones and the subsequent hyperpolarization of OFF CBCs and depolarization of ON CBCs. OFF and ON CBCs synapse with OFF and ON GCs in the outer and inner half of the IPL, respectively. The OFF and ON pathways may enhance the perception of light or dark edges and contrast discrimination (Dolan et al., 1994).

Besides the OFF and ON pathways, the excitatory receptive field (RF) center and inhibitory surround parallel pathways also emerge in the outer plexiform layer. Horizontal cells initiate the inhibitory surround in OFF and ON CBCs to transmit information regarding the size of stimuli in the RF (Hartline, 1940; Enroth-Cugell and Robson, 1966; Werblin and Dowling, 1969; Baylor et al., 1971). The BC center surround organization is modulated and integrated by inhibitory ACs in the IPL so that GCs, which receive input from multiple BCs, also have an overall center surround organization (Kuffler, 1953; Rodieck and Stone, 1965; Lukasiewicz and Werblin, 1990; Werblin, 1991; van Wyk et al., 2009). The processes mediating the formation of the RF center surround organization in GCs are not fully understood. Lateral inhibition from GABAergic ACs enhances the $B C$ center surround organization through $\mathrm{GABA}_{c}$ receptors. However, other spiking GABAergic and glycinergic ACs serially inhibit the ACs providing surround suppression to BCs (Eggers et al., 2013; Moore-Dotson et al., 2015). GABAergic wide-field ACs also provide lateral inhibition directly to GCs to decrease the GC spiking response to stimuli larger than the GC's RF center (Flores-Herr et al., 2001; Eggers et al., 2007; Eggers and Lukasiewicz, 2010; 
Farrow et al., 2013; Protti et al., 2014). Glycinergic narrow-field ACs contribute local inhibition to the GC RF center (Protti et al., 1997; Zhang \& McCall, 2012).

Within the ON and OFF pathways, there is increasing evidence for temporal signaling pathways (Euler et al., 2014). How sustained and transient responses arise from the same cone input is not fully known. There are five known ON CBC and five to ten OFF CBC types in the mouse retina. The glutamate receptors are the same on all ON and OFF types; mGluR6 in ON BCs (Slaughter and Miller, 1985; Nakajima et al., 1993; Morgans et al., 2009; Shen et al., 2009) and non-NMDA-type ionotropic glutamate receptors in OFF BCs (Borghuis et al., 2014, but see DeVries, 2000). Despite that, OFF and ON CBC types have different temporal responses in parallel pathways. OFF and ON sustained BCs stratify in s $1-2$ and s $4-5$ of the IPL, respectively (Awatramani \& Slaughter, 2000; Roska \& Werblin, 2001; Li \& DeVries, 2006; Baden et al., 2013; Euler et al., 2014). Excitatory and inhibitory input, internal properties of $O N$ and OFF BCs, synaptic kinetics and additional processes may shape the temporal properties of glutamate release from CBCs. A combination of sustained or transient release of glutamate from BCs and modulation by ACs contribute to response durations in post-synaptic GCs (see Euler et al., 2014 for a review) and match the duration of the GC spiking response to the stimulus (Borghuis et al., 2013 \& 2014). Sustained responses most likely extract details in a scene (Roska et al., 2006). ON and OFF transient BCs and GCs stratify centrally in the IPL. Transient GCs likely provide the perception of edges and outlines in a scene (Roska et al., 2006). 
Since the glutamate receptors do not differ in ON and OFF CBC types, inhibition from amacrine cells likely modulates the duration of $\mathrm{CBC}$ depolarization and subsequent glutamate output. Slowly responding and long duration $\mathrm{GABA}_{c}$ mediated inhibition decreases the maintained release of glutamate from ON CBCs and shapes the maintained spiking response in ON GCs (Lukasiewicz and Shields 1998; Lukasiewicz et al. 2004; Sagdullaev et al. 2006). GABA $A_{c}$ mediated inhibition shortens the response durations in ACs and GCs in salamander (Dong and Werblin, 1998) and the absence of $\mathrm{GABA}_{c}$ receptors enhances the maintained spiking responses of ON GCs in mouse (Sagdullaev et al., 2006). Tonic GABA release on $\mathrm{GABA}_{c}$ receptors also decreases the spontaneous glutamate release from $\mathrm{ON}$ CBCs, a process that acts as gain control to increase the range of perceptible luminance values (see Chapter 1.2.2.1.c.iv. serial inhibition). $\mathrm{GABA}_{A}$ mediated inhibition decreases the rapid release of glutamate that shapes the peak spiking response in post-synaptic GCs (Eggers \& Lukasiewicz, 2011). ON CBCs do not express glycine receptors (Ivanova et al., 2006). Despite that, glycinergic inhibition enhances excitatory center responses and subsequent ON GC response duration. This modulation occurrs through serial inhibition by glycinergic narrow-field ACs of GABAergic ACs which synapse with ON CBCs or ON GCs. This serial inhibition of inhibition, referred to as disinhibition (Nobles et al., 2012), produces an overall enhancement ON GC spiking responses.

Glutamate release from OFF CBCs is modulated by GABA and glycine (Sagdullaev et al., 2006; Ivanova et al., 2006; Eggers \& Lukasiewicz, 2011). The 
majority of the inhibitory input to OFF CBCs is glycinergic cross-over inhibition from the ON pathway and shapes the rapid and maintained release of glutamate (Ivanova et al., 2006; Eggers \& Lukasiewicz, 2011; Nobles et al., 2012; Borghuis, 2014; Zhang et al., 2014). GABAA mediated inhibition is lower than glycinergic inhibition, but still shapes the rapid release of glutamate. GABAc mediated input provides the lowest amount of inhibition to OFF CBCs. The spontaneous and maintained spiking responses of OFF GCs in mice lacking $G A B A_{c}$ receptors are

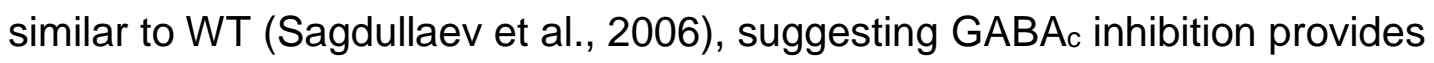
little modulation of maintained glutamate release to some OFF GCs (Eggers \& Lukasiewicz, 2011).

Membrane properties within the ON and OFF GCs modulate the total spiking activity of the GCs in combination with input from BCs and ACs. The spontaneous spiking activity in OFF GCs is internally generated and tonically modulated by glycinergic cross-over inhibition from the ON pathway (Roska \& Werblin, 2001; Zaghloul et al., 2003; Roska et al., 2006; Margolis \& Detwiler, 2007; Chen et al., 2010; Borghuis et al., 2014). In response to a light decrement, cross-over inhibition decreases, which results in an increase in OFF GC spiking activity. The OFF GC response also is enhanced by excitatory input from the OFF CBC. Thus, the peak and maintained response in OFF GCs is shaped by glutamatergic input and rebound spiking activity from release of inhibition. In contrast, tonic glutamate release from the ON CBCs drives the spontaneous spiking in ON GCs (Margolis \& Detwiler, 2007); in the absence of glutamatergic 
input, they do not spike. Thus the peak and maintained response in ON GCs is shaped by excitatory and inhibitory input.

These and other parallel pathways integrate to allow GCs to extract visual information and transmit that signal to the brain for higher order processing. Further complicating matters, however, is the idea that the responses within these pathways may not be static, but depend on stimulus (Umino et al., 2008; Eggers et al., 2013; Farrow et al., 2013; Grimes et al., 2015). For example, some ON GCs can signal light offset and OFF GCs can signal light onset depending on luminance conditions or stimulus size (Sagdullaev \& McCall, 2005; TikidjiHamburyan et al., 2015).

I investigated the contribution of TRPV1 to the rod and cone pathway inputs to GCs. The differences I found between WT and TrpV1\% GCs suggests that TRPV1 shapes visual signals in the following ways:

1. Enhances the luminance threshold of ON and OFF GCs under rod stimulating conditions. TRPV1 likely decreases GABAergic feedback inhibition to RBC in dark adapted conditions.

2. Enhances spontaneous spiking rates in OFF GCs. TRPV1 likely decreases glycinergic inhibition of OFF CBCs and/or GCs.

3. Enhances excitatory input to the RF center of sustained ON GCs to prolong the spiking response. TRPV1 likely decreases GABAc mediated lateral inhibition to ON CBCs in light adapted conditions. 
TRPV1 modulates distinct processes indicative of a role in serial inhibition of GABAergic and glycinergic ACs. Because the center response properties are affected, TRPV1 likely enhances glycine release from narrow-field amacrine cells which modulate local inhibition to the RF center of ON and OFF GCs. In this way, TRPV1 modulates perception by enhancing sensitivity to dim light and discrimination of fine details (visual acuity) in daylight.

\section{Results}

2.1. TRPV1 enhances visual signaling in only the primary rod pathway

Light stimuli near the visual threshold evoke signaling through the primary rod pathway via the All amacrine cell. Rod mediated ERG b-waves are similar between WT and TrpV1\% (Shen et al., 2009), suggesting that TRPV1 does not contribute to the depolarization of the RBC. To determine whether TRPV1 contributes to transmission of visual signal from RBC to GCs through the primary rod pathway, I compared the proportions of dark adapted GCs that responded to the lowest luminance full-field stimulus $\left(0.6 \log R^{*} / \mathrm{rod} / \mathrm{sec}\right)$ in WT ( $n=10$ retina pieces from 5 mice) and $\operatorname{TrpV} 1 \%(\mathrm{n}=9$ retina pieces from 5 mice) retinas, recorded using a multi-electrode array (MEA). I found that neither genotype had ON/OFF responses to the dimmest light. Fourteen percent of WT GCs responded $(\mathrm{n}=81)$ and significantly fewer $\operatorname{TrpV1\% }{ }^{-} \mathrm{GCs}$ were responsive to the same stimulus (Fig. $3.1 ; 1 \%, n=5, p<0.001$ ). Because of the difference in the 
proportions of WT and $\operatorname{TrpV} 1 / \mathrm{GCs}$ responsive to $0.6 \log \mathrm{R}^{\star} / \mathrm{rod} / \mathrm{sec}$ stimulus, I concluded that TRPV1 is necessary for signaling through the primary rod pathway and sets the sensitivity of the rod driven GC light responses.

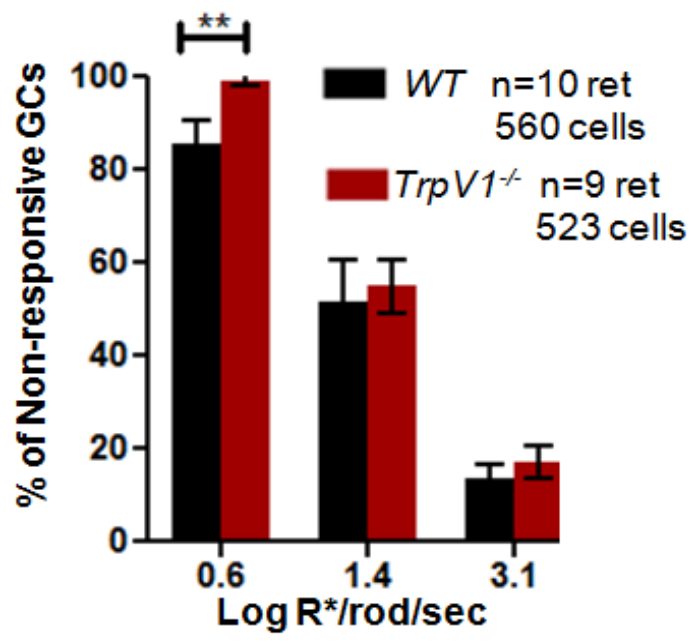

Figure 3.1 Higher luminance is required to evoke a response in $\operatorname{TrpV1}^{-/}$(A) Full-field luminance increments were presented to dark adapted WT $(\mathrm{n}=10)$ and TrpV1\% $(n=9)$ retinas on the MEA. The proportions of nonresponsive (NR) GCs were compared between WT ( $n=560$ GCs) and $\operatorname{TrpV1} \%(\mathrm{n}=523 \mathrm{GCs})$ at each luminance. The first increment $\left(0.6 \log R^{*} / \mathrm{rod} / \mathrm{sec}\right)$ isolated signals through the primary rod pathway. The majority of WT GCs were $N R$ at the first luminance $(n=479,86 \pm 5 \%)$ and there was a larger proportion of $\operatorname{TrpV1} 1-\mathrm{NR}$ GCs $\left(\mathrm{n}=526,99_{ \pm} 1 \%\right.$;

ANOVA $p=0.02$, post hoc Bonferroni t-test $p<0.01$ ) compared to WT. Eighty-one WT and five TrpV1-GCs had responses mediated through the primary rod pathway, indicating TRPV1 was necessary for signaling through the RBC-All AC. The second luminance (1.4 log $\left.\mathrm{R}^{*} / \mathrm{rod} / \mathrm{sec}\right)$ evoked signaling through the secondary rod and cone pathways. The third luminance $\left(3.1 \log \mathrm{R}^{*} / \mathrm{rod} / \mathrm{sec}\right)$, evoked signaling through the tertiary rod and cone pathways. Proportions of WT NR GCs decreased with increasing luminance $(52 \pm 9 \% \& 14 \pm 3 \%$, respectively). There were similar proportions of WT and TrpV1/-NR GCs at the second $(55 \pm 6 \%)$ and third $(17 \pm 4 \%)$ luminance increments. TRPV1 did not contribute to secondary or tertiary rod signaling. Values were reported as mean \pm sem.
To determine the level of the disruption within the rod pathway, I examined the differences in the responses of WT and TrpV1\% GCs under luminance conditions that stimulated the three rod pathways. After the presentation of the dimmest luminance, I presented two luminance increments (1.4

\& 3.1 Log $\mathrm{R}^{*} / \mathrm{rod} / \mathrm{sec}$ ) reported to elicit responses mediated by rod and cone pathways (Völgyi et al., 2004). The percent of responsive WT GCs increased with luminance. Similar proportions of WT and TrpV1- GCs had responses under luminance conditions that stimulated the secondary and tertiary 
pathways (Fig. 3.1). This result suggests TRPV1 plays a role in enhancing the sensitivity of the primary rod pathway to threshold stimuli. The ERG b-wave represents the depolarization of the ON BCs and is similar between $\operatorname{TrpV1\% }$ and WT mice in response to all tested luminance values (Shen et al., 2009). TRPV1 must enhance glutamate transmission to the All or regulate transmission of the signal through the All directly.

\subsection{TRPV1 does not contribute to primary cone pathway signaling}

Cone mediated ERG b-waves are similar between WT and TrpV1-1 (Shen et al., 2009), suggesting visual signal transmission through the ON CBCs is intact. To determine if TRPV1 contributed to transmission of visual signal through the cone pathway, I assessed light evoked spiking responses in WT and TrpV1\% GCs under light adapted conditions. To compare temporal and spatial processing confined to the RF center or surround, visually evoked spiking responses were recorded from individual GC axons in the optic nerves of anesthetized TrpV1/ and WT mice (Fig. 3.2). To evoke RF center dominated response, spots of varying size were presented above a light adapting background (4 log $\mathrm{R}^{*} / \mathrm{rod} / \mathrm{sec}$ ) confined to the RF center of individual GCs. An area response function (ARF) plotting amplitude of response as a function of spot size was computed for each GC and the spot size corresponding to its peak, which represents the spatial summation of all excitatory inputs, defined the RF center diameter (see Methods). GCs were classified as OFF, ON or ON/OFF according to the response to a dark (3 $\left.\log \mathrm{R}^{\star} / \mathrm{rod} / \mathrm{sec}\right)$ or light $\left(4.7 \log \mathrm{R}^{\star} / \mathrm{rod} / \mathrm{sec}\right)$ spot 
whose diameter matched the RF center diameter. Figure 3.2 shows representative raster plots and PSTHs for sustained ON and OFF GCs in

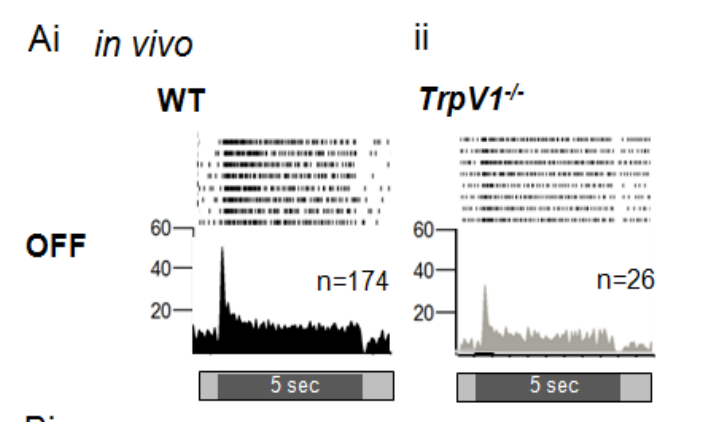

$\mathrm{Bi}$

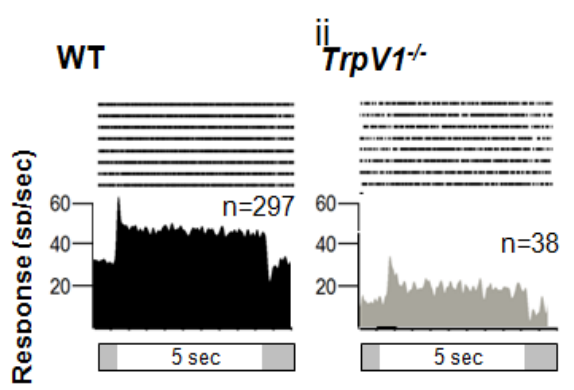

C

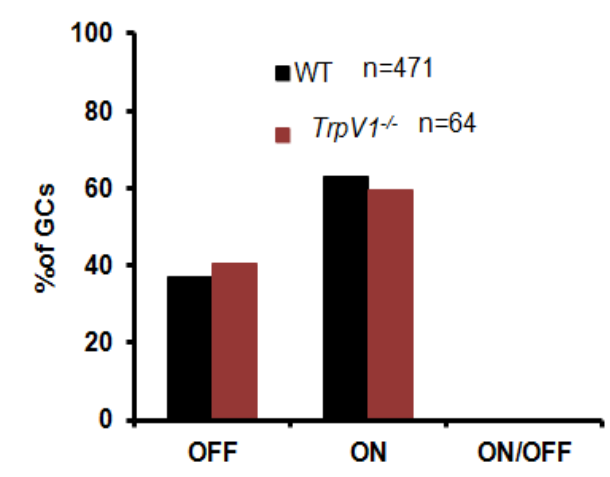

Figure 3.2 TRPV1 does not contribute to cone pathway signaling in GCs sampled in vivo. (A) ON and OFF alpha GCs are sampled in vivo. $A$ and $B$ show representative PSTHs for WT (Ai \& Bi) and TrpV1\%(Aii \& Bii) OFF (A) and ON (B) GCs in response to a dark spot (3.0 log $\left.R^{\star} / \mathrm{rod} / \mathrm{sec}\right)$ or light $(4.7 \mathrm{log}$ $\mathrm{R}^{*} / \mathrm{rod} / \mathrm{sec}$ ) spot, respectively, whose diameter matched the receptive field center. (C) A larger proportion of ON center GCs $(63 \%)$ than OFF GCs (37\%) was sampled in the WT in vivo. Similar proportions of WT and TrpV1\% ON (60\%) and OFF (40\%) GCs were sampled in vivo (Fisher's exact test, $\mathrm{p}=0.58$ ). ON/OFF GCs were not sampled in either genotype in vivo. In the absence of TRPV1, visual signal transmission was intact through the ON and OFF cone pathways to ON and OFF alpha GCs

WT and TrpV1\%. The proportion of WT ON GCs was greater than WT OFF GCs. The proportion of $\operatorname{TrpV1}{ }^{-}$ON and OFF GCs was similar to WT (Fisher's exact test $p=0.58$ ). As we have observed before, ON/OFF GCs were not sampled in optic nerve recordings (Nobles et al., 2012).

The morphological identity of the WT and TrpV1\% GCs sampled in optic nerve and MEA recordings are unknown. In vivo optic nerve recordings target 
GCs with large axon diameters (Boycott and Wässle, 1974). Given the brisk responses of ON and OFF GCs, we likely record from ONa and OFFa GC

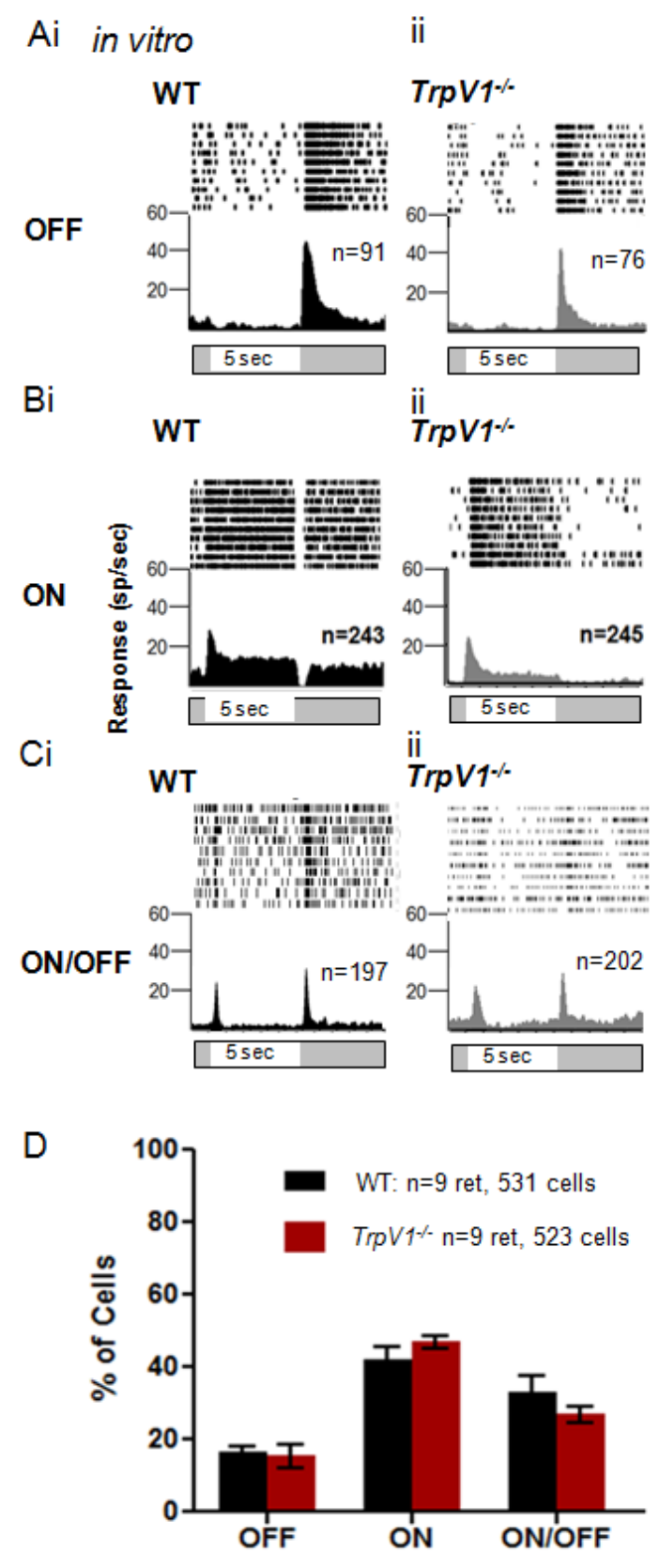

Figure 3.3 TRPV1 does not contribute to cone pathway signaling in GCs sampled in vitro. (A) More GC classes are sampled using the MEA in vitro than in vivo. $A B$ and $C$ show representative PSTHs for WT (Ai, Bi \& Ci) and TrpV1- (Aii, Bii \& Cii) OFF (A) ON (B) and ON/OFF (C) GCs in response to in vitro cone stimulating full-field luminance step (4.1 $\log R^{*} /$ rod/sec) above a light adapting background (2.6 log $R^{*} / \mathrm{rod} / \mathrm{sec}$ ) (C) The proportions of each $\mathrm{GC}$ class was determined in each retinal piece $(n=9)$ and the mean percent compared between WT $(n=9)$ and $\operatorname{TrpV1}{ }^{-\%}(\mathrm{n}=9)$. The largest proportion WT GCs sampled had ON responses (46\%), then ON/OFF (37\%) and the smallest proportion were OFF $(17 \%)$. Similar proportions of WT and $\operatorname{TrpV} 1 \%$ ON (47\%), ON/OFF (39\%) and OFF (15\%) GCs were sampled in vitro. (ANOVA, $p=0.71$ ). In the absence of TRPV1, visual signal transmission was intact through the ON and OFF cone pathways to all GC classes sampled in vitro.

classes (Manookin et al., 2008) or A-type (Sun et al. 2002) which have large somas, dendritic fields and axon diameters (Kuffler, 1953; Enroth-Cugell and Robson, 1966; Manookin et al., 2008; Nobles et al., 2012). To confirm my results 
among other GC classes, I recorded from WT and TrpV1\% GCs using the MEA in vitro, which likely samples more GCs classes than in vivo (Fig. 3.3; Anishchenko et al., 2010; Marre, et al., 2012; Li et al., 2015; Walch et al., 2015). I compared the proportions of GCs that responded under light adapted conditions to 4.1 log $\mathrm{R}^{*} / \mathrm{rod} / \mathrm{sec}$ in WT ( $\mathrm{n}=9$ retina pieces from 5 mice) and $\operatorname{TrpV1}{ }^{-1}(\mathrm{n}=9$ retina pieces from 5 mice) retina (Fig. 3.3). Similar to my in vivo results, the majority of WT GCs had ON (Fig. 3.3bi \& d) responses and a smaller proportion had OFF (Fig. 3.3ai \& d). In contrast to my in vivo results, $37 \%$ of WT GCs were ON/OFF (Fig. 3.3ci \& d). TrpV1\% and WT GCs had similar proportions of OFF, ON and ON/OFF (Fig. 3.3d, ANOVA $p=0.71$ ) responses. Full field luminance increments stimulated both the RF center (excitation) and surround (suppression) and the response represents the combination of their excitation and suppression (Kuffler 1951). OFF and ON center GCs can gain excitatory responses of the opposite sign (ON and OFF, respectively) to full-field stimuli (Sagdullaev \& McCall, 2005; Tikidji-Hamburyan et al., 2015). Despite this, cone signaling through the ON and OFF pathways occurs in similar proportions in WT and TrpV1-- to full-field stimuli. The distributions of RF center classes were not affected by the absence of TRPV1. From this I concluded TRPV1 did not influence the primary cone pathway. To assess the contribution of TRPV1 to the ON and OFF pathways individually, I compared spontaneous and light evoked spiking activity in WT and $\operatorname{TrpV} 1 \% \mathrm{ON}$ and OFF GCs. The origin of the excitatory spiking responses in ON/OFF GCs is more complex and can be elicited by excitatory or inhibitory 
input in response to full-field stimuli (Sagdullaev \& McCall, 2005). Therefore, response properties of the ON/OFF GC are not discussed.

\subsection{TRPV1 enhances spontaneous activity in OFF GCs}

I compared spontaneous activity in the dark and with a light adapting background. Due to the large numbers of GCs recorded on the MEA from few mice, $\alpha \leq 0.01$ was considered significant for all comparisons (see Appendix I).
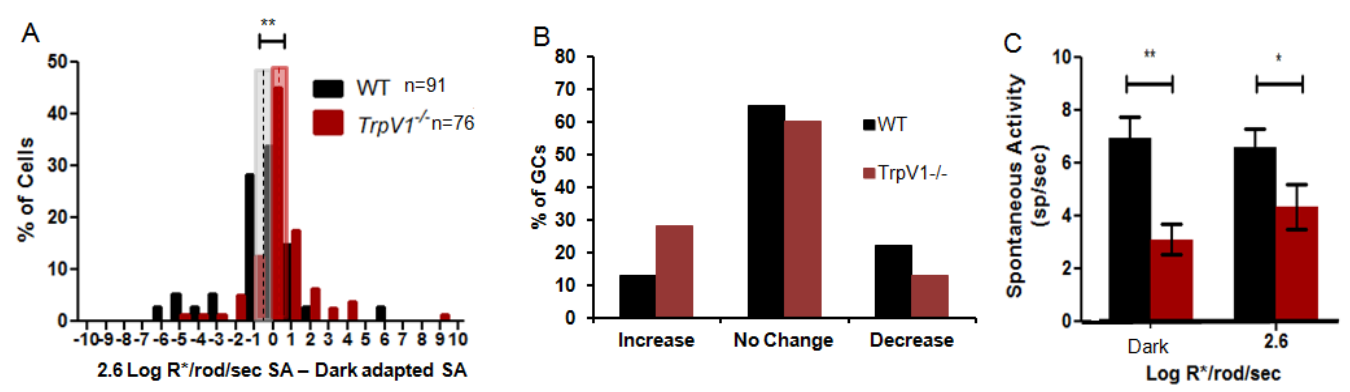

Figure 3.4 Spontaneous activity was lower in TrpV1/- OFF GCs (Ai) WT OFF GC spontaneous rates were similar under dark $(6.7 \pm 1.3 \mathrm{sp} / \mathrm{sec})$ and light adapted $(6.2 \pm 1.1 \mathrm{sp} / \mathrm{sec}$; $\mathrm{p}=0.95)$ conditions. TrpV1/ mean spontaneous rates also were similar under dark $(2.7 \pm 0.7$ $\mathrm{sp} / \mathrm{sec})$ and light adapted $(3.1 \pm 0.7 \mathrm{sp} / \mathrm{sec} ; \mathrm{p}=0.65)$ conditions. The spontaneous in the dark was subtracted from the spontaneous activity in light conditions. The distributions of the change in spontaneous activity along with the mean (dotted line) and three standard errors (shaded region) where determined for WT $(-0.6 \pm 0.2)$ and TrpV1 $1-(0.3 \pm 0.1)$ OFF GCs. The change in spontaneous activity was significantly larger in TrpV1- compared to WT $(\mathrm{p}=0.008)$. The spontaneous activity of the majority of WT OFF GCs did not change $(68 \%)$, while $11 \%$ increased and $21 \%$ decreased (B). The proportions of TrpV1- OFF GC with changes in spontaneous activity was similar to WT (Chi square; $p=0.04)$. The majority $(60 \%)$ did not change, 30\% increased and 10\% decreased spontaneous activity in the light. TRPV1 did not contribute to the processes which maintained the similar spontaneous spiking rate in the light. In contrast, the mean spontaneous activity was decreased in TrpV1\%OFF GCs in both dark and light adapted conditions compared to WT $(C ; p<0.0001)$. TRPV1 enhances the spontaneous activity in OFF GCs.

The WT OFF GC mean spontaneous rates were similar under dark $(6.7 \pm 1.3$ $\mathrm{sp} / \mathrm{sec})$ and light adapted $(6.2 \pm 1.1 \mathrm{sp} / \mathrm{sec} ; \mathrm{p}=0.95)$ conditions (Fig. 3.4a), suggesting little modulation of spontaneous activity by light in OFF GCs. For each WT and TrpV1- OFF GC, I determined the change in spontaneous activity 
with a 2.6 Log $\mathrm{R}^{*} / \mathrm{rod} / \mathrm{sec}$ background from dark. I plotted the difference in spontaneous activity in a histogram along with the mean difference (dotted line) plus and minus three standard errors of the mean (shaded region) for each genotype. I found populations of WT OFF GCs whose spontaneous activity increased, decreased, or remained the same in the light (Fig. 3.4b), however the mean change was zero (Fig. 3.4a). Figure 3.4a shows the distributions of the WT and $\operatorname{TrpV1} \%$ OFF GCs also were similar under dark $(2.7 \pm 0.7 \mathrm{sp} / \mathrm{sec})$ and light adapted $(3.1 \pm 0.7 \mathrm{sp} / \mathrm{sec} ; \mathrm{p}=0.65)$ conditions, however the mean change in spontaneous activity in the light was significantly increased compared to WT $(0.3 \pm 0.1 \mathrm{sp} / \mathrm{sec} ; \mathrm{p}=0.008)$. This suggests TRPV1 may contribute to the processes underlying the spontaneous activity in OFF GCs. The proportions of TrpV11-OFF GCs that had increased or decreased spontaneous activity in the light compared to the dark was similar to WT (Fig. 3.4b; chi square $p=0.04$ ). Spontaneous activity of TrpV1\% OFF GCs was significantly lower than WT at both adaptation levels (Fig. 3.4c; $p<0.0001$ ). TRPV1 enhances spontaneous activity in OFF GCs. Spontaneous activity is internally generated in OFF GCs which have sustained (sOFF) and transient (tOFF) responses (Margolis \& Detwiler, 2007). Under light adapted conditions, OFF GC spontaneous activity is strongly inhibited by glycinergic cross-over inhibition (Margolis \& Detwiler, 2007; Nobles et al., 2012). The inputs shaping spontaneous activity in OFF GCs in the absence of light is not well known, however cross-over inhibition from the All may continue to drive much of the inhibitory synaptic input to the OFF GC (Murphy and Rieke, 2008). Lower spontaneous activity in the $\operatorname{TrpV1} 1 /$ most likely represents increased 
inhibition on OFF GCs in both conditions. The following could explain the difference:

1. TRPV1 could enhance inhibition onto Alls or other cross-over ACs, which make synaptic connections with OFF GCs. In the absence of TRPV1, the AC becomes more active and inhibits the OFF BCs and GCs more. If this were true, the lack of transmission through the primary rod pathway would have to come from increased inhibition of the RBC

2. TRPV1 could inhibit the signal from the ON pathway to the cross-over
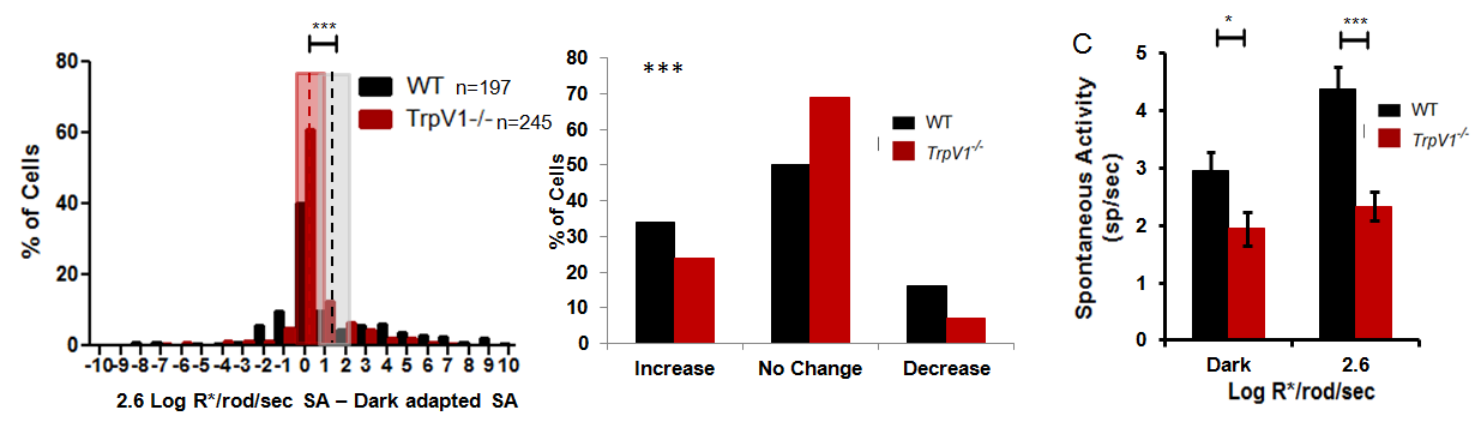

Figure 3.5 Spontaneous activity is lower in TrpV1/- ON GCs and do not increase with light (A) WT ON GC spontaneous rates were higher under light adapted conditions $(4.3 \pm 0.37 \mathrm{sp} / \mathrm{sec})$ compared to dark $(3.0 \pm 0.32 \mathrm{sp} / \mathrm{sec}$; paired t-test $\mathrm{p}<0.0001)$ conditions. In contrast, $\bar{T} r p V 1 \% \mathrm{ON}$ GC mean spontaneous rates were similar under dark $(2.0 \pm 0.3 \mathrm{sp} / \mathrm{sec})$ and light adapted $(2.3 \pm 0.3$ $\mathrm{sp} / \mathrm{sec}$; paired t-test $\mathrm{p}=0.32$ ) conditions. The spontaneous rate in the dark was subtracted from the spontaneous rate in light conditions. The distributions of the change in spontaneous activity along with the mean (dotted line) and three standard errors (shaded region) where determined for WT $(1.4 \pm 0.3)$ and TrpV1- $(0.4 \pm 0.2)$ ON GCs. The change in spontaneous activity was significantly larger in WT compared to $\operatorname{TrpV1} \%(\mathrm{p}=0.0009)$. The spontaneous activity of half of WT ON GCs did not change (50\%), while $34 \%$ increased and $16 \%$ decreased in light (B). The proportions of $\operatorname{TrpV1} 1$-OFF GC with changes in spontaneous activity was lower than WT (Chi square; $p=0.0002)$. The majority $(69 \%)$ did not change, $24 \%$ increased and $7 \%$ decreased spontaneous activity in the light. TRPV1 enhanced the ON GC change in spontaneous activity to light. The mean spontaneous activity was decreased in TrpV1\%ON GCs in both dark and light adapted conditions compared to WT $(C ; p<0.0001)$. TRPV1 enhances the spontaneous activity in ON GCs.

AC. In the absence of TRPV1, ON input to the AC is enhanced and so is crossover inhibition. The latter is inconsistent with lack of signal from the primary rod 
pathway (Nobles et al. 2012). TRPV1 activity may affect the dark contributing AC more than the cross-over AC. Alternatively, if TRPV1 resides in OFF GCs, it may affect the spiking activity directly.

3. TRPV1 enhances glutamate release from OFF BCs. In the absence of TRPV1, excitatory input to the OFF GC is decreased and spontaneous activity is decreased. If this were true, TRPV1 may be regulating inhibition to the OFF BC. This would likely be through serial inhibition of another AC, which provides feedback or feed forward inhibition to the OFF BC or GC.

\subsection{TRPV1 enhances spontaneous activity in ON GCs}

I made similar comparisons of the spontaneous activity in WT and TrpV1ON GCs. The WT ON GC mean spontaneous rates increased with light adaptation (Fig 3.5; $p=0.95$ ), consistent with previous publications (cat- Barlow and Levick, 1969; mouse- Sagdullaev \& McCall, 2005; Zaghloul et al., 2003). Figure 3.5a shows the distributions of the change in spontaneous activity for WT and $\operatorname{TrpV} 1 \%$ ON GCs. I found populations of WT ON GCs whose spontaneous activity increased, decreased, or remained the same in the light (Fig. 3.5a \& b). Among the WT ON GCs, 34\% had increased spontaneous activity in 2.6 log $\mathrm{R}^{\star} / \mathrm{rod} / \mathrm{sec}$ light conditions compared to dark. A minor population (16\%) had decreased spontaneous activity, and $50 \%$ had no change (Fig. 3.5b). In contrast, the mean spontaneous activity of TrpV1 1 ON GCs were similar under dark $(2.0 \pm 0.3 \mathrm{sp} / \mathrm{sec})$ and light adapted $(2.3 \pm 0.3 \mathrm{sp} / \mathrm{sec}$; paired t-test $\mathrm{p}=0.65)$ conditions. The mean change in spontaneous activity in the light was significantly lower compared to WT (Fig. 3.5a; $p<0.0001$ ). There was no change in 
spontaneous activity under light adapted conditions in $69 \%$ of $\operatorname{TrpV} 1 \%$ ON GCs and the proportions of $\operatorname{TrpV} 1 \% \mathrm{ON}$ GCs that had increased or decreased spontaneous activity in the light compared to the dark was lower than WT (Fig. $3.5 \mathrm{~b}$; Chi square $\mathrm{p}=0.0002$ ). TRPV1 is required for light induced modulation of spontaneous activity in $\sim 20 \%$ of ON GCs. Spontaneous activity of $\operatorname{TrpV} 1 \%$ ON GCs was significantly lower than WT at both adaptation levels (Fig. 3.5c; ANOVA $\mathrm{p}<0.0001)$. TRPV1 enhances spontaneous activity in ON GCs.

Spontaneous activity in ONa GCs is driven by glutamate release from ON CBCs (Massey et al., 1983; Zaghloul et al., 2003; Margolis \& Detwiler, 2007). In the dark, spontaneous glutamate release and ON GC spike rate is low. Under light adapted conditions, glutamatergic input and spiking rate increases. Inhibition to the ONa GC plays little role in shaping spontaneous activity (Margolis \& Detwiler, 2007). My data shows that spontaneous activity in 50\% of WT ON GCs and $\sim 30 \%$ of $\operatorname{TrpV1}^{-1-}$ were modulated by light (Fig. 3.5b). In the absence of TRPV1, equal proportions ( 10\%) of ON GCs that increased or decreased spontaneous activity with light lost light modulation. Also the mean spontaneous rates were lower under dark and light adapted conditions. This suggests TRPV1 may regulate tonic inhibition to the $\mathrm{ON} \mathrm{BC}$ or ON GC. GABAergic inhibition to $\mathrm{GABA}_{c}$ receptors on ON CBCs decreases spontaneous glutamate release to ON GCs (Sagdullaev et al., 2006). Also, glycine regulates ON GC spontaneous activity through serial inhibition (Nobles et al., 2012). TRPV1 may play a role in glycinergic serial inhibition of $\mathrm{GABA}_{c}$ feedback inhibition. In this case, the absence of TRPV1 tonically disinhibits the GABAergic feedback AC, leading to 
increased GABA release onto the ON CBC and subsequent decreased glutamate release from the ON CBC. Alternatively, TRPV1 may regulate inhibition to the ON GC directly or, if TRPV1resides in ON GCs, it may regulate spiking activity there. In summary, the lower spontaneous activity in the absence of TRPV1 may be the result of:

1. Decreased tonic glutamate release from ON CBCs.

2. Increased tonic inhibition to the ON GCs.

3. Changes in the ON GCs that decreased the spike rate.

I next assessed light evoked spiking responses address each of these possible roles for TRPV1. Because spontaneous activity was different between WT and $\operatorname{TrpV} 1 \%$ and changes with stimulus conditions, spontaneous activity was subtracted from all light evoked responses.

2.5 TRPV1 prolongs the duration of ON and regulates duration in OFF GC responses in vitro.

In the absence of TRPV1, rod mediated signal through the RBC-All AC circuit is disrupted, while cone mediated signal to OFF and ON GCs is maintained. Within OFF and ON cone pathway, there are sustained and transient temporal parallel pathways that culminate in GC responses to the onset of a 


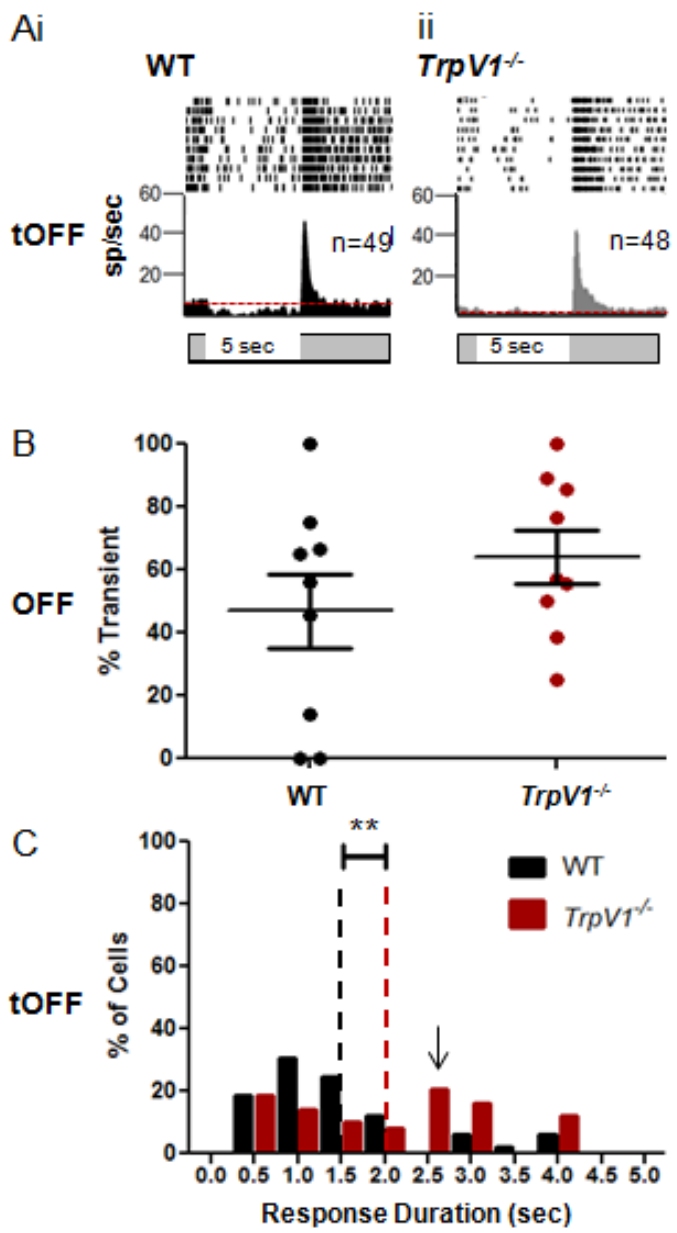

Figure 3.6 OFF response durations were prolonged in TrpV1 ${ }^{-/}$tOFF GCs to photopic stimuli (A) Representative PSTHs of WT $(\mathrm{n}=49)$ and $\operatorname{TrpV1} 1 \mathrm{n}=48)$ transient OFF (tOFF) GCs. (B) The percent of transient responses among OFF GCs was determined in WT $(n=9)$ and $\operatorname{TrpV1} \%(n=9)$ retinal pieces. The mean percent of tOFF GCs per retinal piece was similar between WT $(47 \pm 12 \%)$ and TrpV1- $(64 \pm 8 \%, p=0.25)$ retinas. (C) The majority of WT tOFF GCs had durations $<2$ s to the offset of $4.1(n=40,81 \%) \log R^{\star} / \mathrm{rod} / \mathrm{sec}$ stimulation (B) Nineteen percent of WT tOFF GCs had response durations $3>4 s(n=9)$. Twenty-four (51\%) TrpV1- tOFF GCs had durations <2s to the offset of $4.1 \log R^{*} / \mathrm{rod} / \mathrm{sec}$ stimulation. Similar to WT, $28 \%(n=13)$ of TrpV1\% tOFF GCs had response durations $3>4 \mathrm{~s}$. In contrast to WT, $21 \%(n=11)$ of $T r p V 1 \%$ tOFF GCs had a response phenotype not observed in WT. These GCs responded for $2>3 \mathrm{~s}$ (arrow). (C) TrpV1- tOFF mean response durations were greater than WT tOFF GCs $(p<0.01)$.

stimulus (transient) or its maintained presence (sustained). I examined the sustained or transient pathway by comparing their responses in WT and TrpV1\% under cone pathway mediated luminance conditions; a $4.1 \log R^{\star} / \mathrm{rod} / \mathrm{sec}$ luminance increment (5 s) above a $2.6 \log R^{\star} / \mathrm{rod} / \mathrm{sec}$ background. Responses to this stimulus paradigm were analyzed for the remainder of the in vitro comparison. I found that almost half of WT OFF GCs within retinas had transient responses (Fig. 3.6b; tOFF; 47\%). The proportions of TrpV1- tOFF GCs were similar to WT (Fig. 3.6b; 64\%; $p=0.25$ ). I conclude that TRPV1 does not directly 


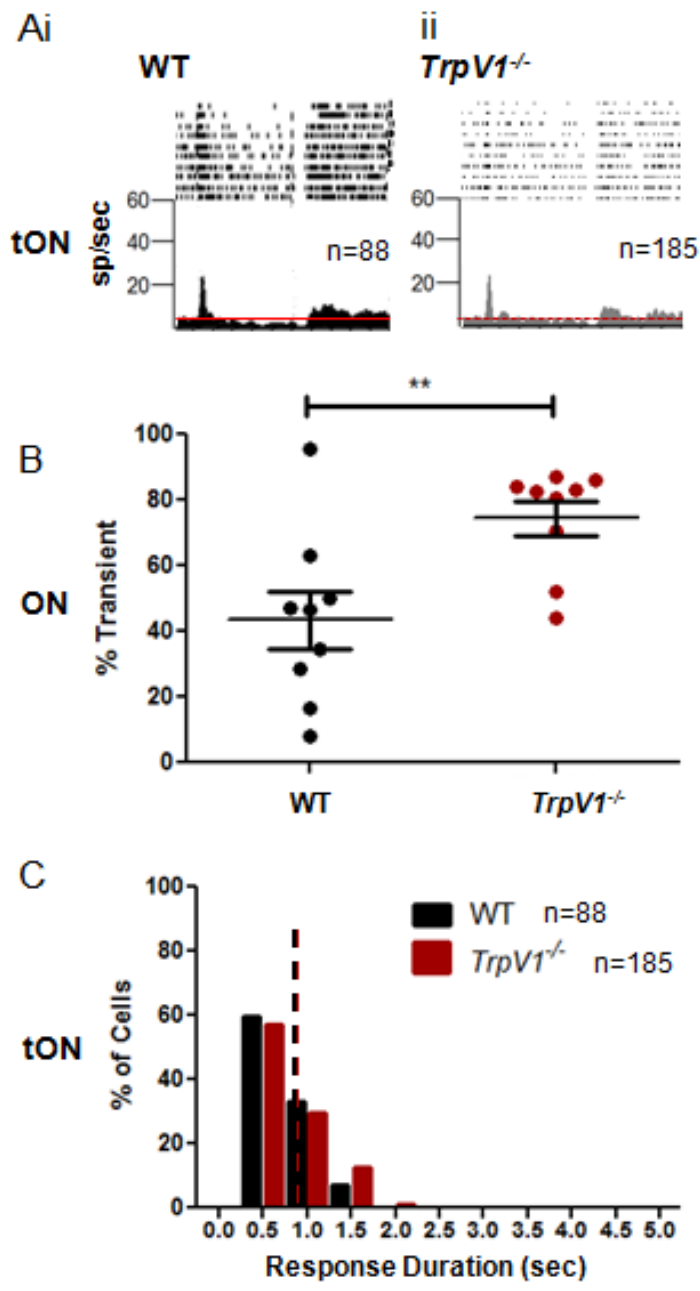

Figure 3.7 OFF response durations were prolonged in TrpV1 $1 /-$ tOFF GCs to photopic stimuli (A) Representative PSTHs of WT (Ai; $\mathrm{n}=88)$ and $\operatorname{TrpV1} \%($ Aii; $\mathrm{n}=185)$ transient $\mathrm{ON}$ (tON) GCs. (B) The percent of transient responses among ON GCs was determined in WT $(n=9)$ and $\operatorname{TrpV1}{ }^{-1}(\mathrm{n}=9)$ retinal pieces. The mean percent of tON GCs per retinal piece was larger in TrpV1\% $(74 \pm 5 \%)$ compared to WT $(43 \pm 9 \%, p=0.008)$. (C) All WT and TrpV1-1 tON GCs had durations $<2 \mathrm{~s}$ to the onset of $4.1 \log R^{*} / \mathrm{rod} / \mathrm{sec}$ stimulation. $\operatorname{TrpV1} 1-\mathrm{tON}$ mean response durations were similar to WT tON GCs $(p=0.76)$.

regulate temporal kinetics of OFF GCs that match their response to stimulus duration.

Among the WT tOFF GCs, there were two distinct populations based on response duration. Out of 49 WT tOFF GCs $81 \%$ had responses shorter than $2 \mathrm{~s}$ and the remaining 19\% had responses between 3 and $5 \mathrm{~s}$ (Fig. 3.6c). No WT OFF GCs had responses $2>3 \mathrm{~s}$. A smaller proportion of TrpV1\% tOFF GCs (51\%) than WT had responses shorter than $2 \mathrm{~s}$ (Fig. 3.6c). Similar to WT, $28 \%$ of TrpV1- tOFF GCs had response durations between 3 and $5 \mathrm{~s}$. The remaining $21 \%$ of $\operatorname{TrpV} 1 \%$ tOFF GCs had responses $2>3 \mathrm{~s}$ (Fig. 3.6c arrow), which was 
not a phenotype seen in WT. The mean response duration was longer in TrpV1/tOFF GCs compare to WT (Fig. 3.6c; $p=0.01$ ). From this I conclude that TRPV1 regulates response durations to cone mediated signals in $\sim 21 \%$ of tOFF GCs. There are $20 \%$ more tOFF GCs sampled in TrpV1/- compared to WT. That is not a statistically significant difference, however I cannot determine whether TRPV1 prolongs or shortens the response in this small population of OFF GCs.

Figure 3.7a shows representative PSTHs of WT and TrpV1- transient ON (tON) GCs. Among the WT ON GCs 43\% within retinas had transient responses (tON) at $4.1 \log \mathrm{R}^{*} / \mathrm{rod} / \mathrm{sec}$. In contrast to $\operatorname{TrpV1} 1$ OFF GCs, TrpV1- ON GCs were more transient than WT (Fig 3.7b; 74\%; $p=0.008$ ). TRPV1 increases the response durations to cone mediated ON signals in $\sim 31 \%$ of ON GCs and in $\sim 54 \%$ of ON GCs that have sustained responses (sON). I conclude that TRPV1 is required to regulate temporal kinetics of ON GCs that match their response to stimulus duration.

WT tON GCs, had response duration $<1.7 \mathrm{~s}$ with mean $0.71 \mathrm{~s}$. The distribution and mean of the $\operatorname{Trp} V 1 \%$ tON GC response durations were similar to WT (Fig 3.7c; $p=0.76$ ). I could not determine the specific sON GCs that have shortened durations in the absence of TRPV1 by their response durations or other response properties (shown later). In order to make direct comparisons between similar GC classes, ON GCs were combined and compared as one functional class.

2.6 TRPV1 contributes to the maintained response of OFF and ON GCs 
I compared light responses in cone mediated luminance conditions between WT and TrpV1\% ON and OFF GCs to determine if TRPV1 modulates response properties. Light responses are composed of a transient increase in spike rate, followed by a maintained response that returns to prestimulus baseline spiking at variable times corresponding to stimulus duration.
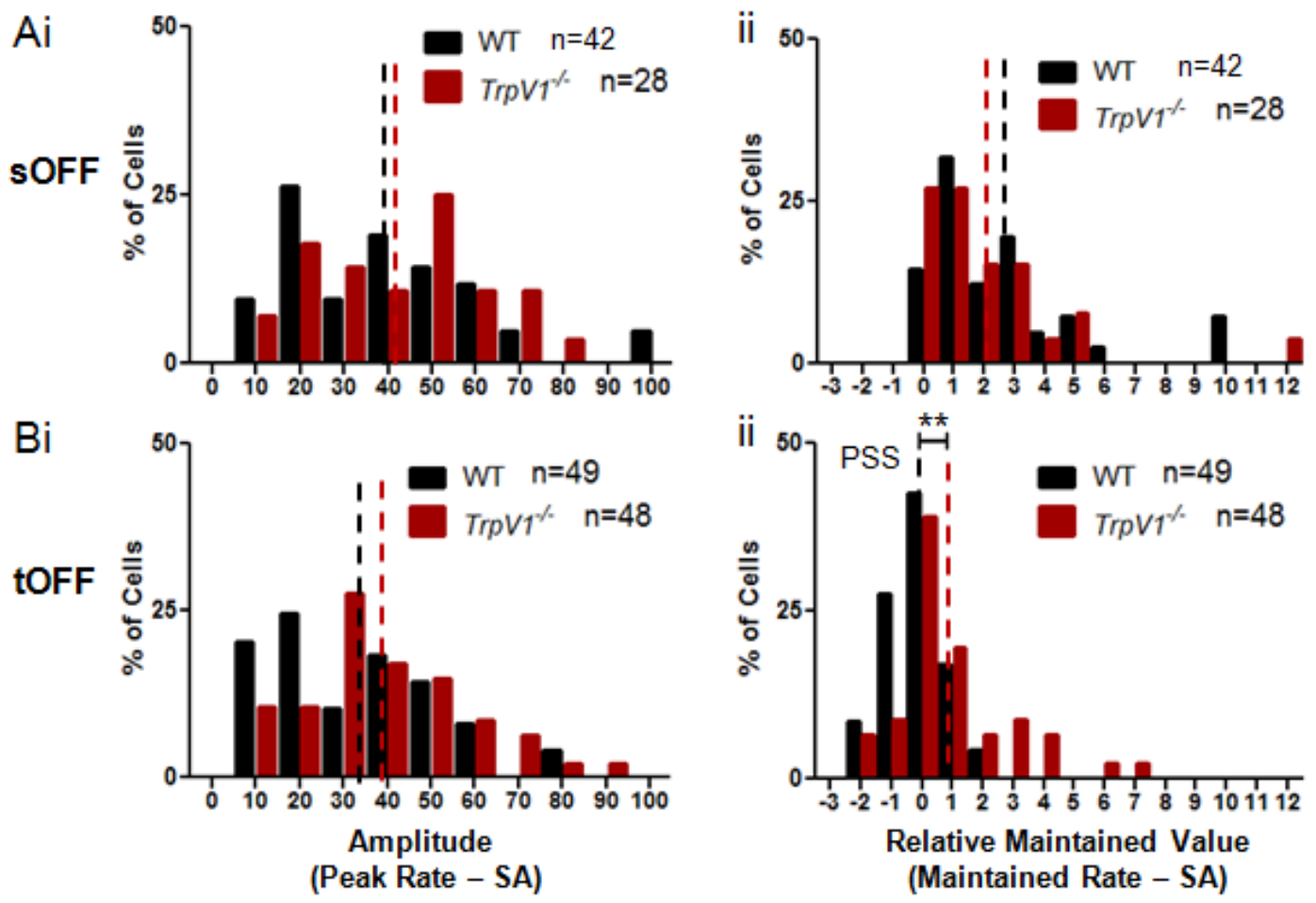

Figure 3.8 Maintained response rates were increased in TrpV1\% tOFF GCs compared to WT. (Ai) The proportions of tOFF GCs were similar between WT and TrpV1\% OFF GCs. I compared the peak amplitude (i) and maintained rates (ii; corrected for spontaneous activity) in WT and $\operatorname{TrpV1}$ - sOFF (A) and tOFF (B) GCs. Amplitudes (Ai) were similar between WT $(39.1 \pm 3.3)$ TrpV1\% $(42.8 \pm 3.8)$ sOFF GCs $(p=0.37)$. (Aii) Relative maintained values also were similar between WT $(2.6 \pm 0.4) \operatorname{TrpV1} \%(2.1 \pm 0.5)$ sOFF GCs $(\mathrm{p}=0.46)$. (Bi) Amplitudes were similar between WT (33.3 \pm 2.6$)$ TrpV1\% $(-0.1 \pm 0.1)$ tOFF GCs $(p=0.001)$. (Bii) The mean relative maintained value of TrpV1- tOFF GCs $(0.9 \pm 0.3)$ was larger than WT- $(-0.1 \pm 0.1 ; p<0.001)$. TRPV1 does not contribute to the processes shaping the peak of the response, but regulates the maintained portion and duration of response of OFF GCs.

The transient portion of the GC response to a full- field stimulus is shaped primarily from the excitatory inputs of the presynaptic BCs with some modulation 
by AC mediated inhibition (Eggers \& Lukasiewicz, 2011). Thus, an analysis of peak response can help to define if TRPV1 plays a role in modulation of the BC input to each GC type. The maintained portion of the response is likely shaped by a combination of excitatory and inhibitory inputs, with $\mathrm{GABA}_{c}$ mediated inhibition modulating ON GC maintained activity. I assessed the peak and maintained rates in response to light to determine if response properties were affected by the absence of TRPV1. Spontaneous activity was subtracted from peak and maintained rates because of the difference between WT and $\operatorname{TrpV} 1^{-1}$. The corrected peak was referred to as the amplitude (Fig. 3.8) and the corrected maintained response was referred to as the relative maintained value in all subsequent analysis (Fig. 3.8aii \& bii). Sustained GCs had positive relative maintained value, while transient GCs had relative maintained values less than, equal, or greater than zero. Among the transient GCs, relative maintained values less than zero indicate suppression of the response below spontaneous activity, which is referred to as the peri-stimulus suppression (PSS; Fig. 3.8bii).

I compared the peak response amplitude in WT and TrpV1\% OFF GCs. The distribution and mean of TrpV1\% sOFF GC amplitudes was similar to WT (Fig. 3.8ai; $p=0.37$ ). WT and TrpV1\% sOFF GC relative maintained values also were similar (Fig. 3.8aii; $p=0.46$ ) suggesting TRPV1 does not contribute to the peak or maintained response of sOFF GCs. The distribution and mean of $\operatorname{TrpV1/-}$ tOFF GC amplitudes was similar to WT (Fig. 3.8bi; $p=0.17$ ). From this, I conclude that TRPV1 does not contribute to the peak response of OFF GCs, which is shaped by glutamate release from OFF BCs and the release of cross-over 
inhibition from the ON pathway (Margolis \& Detwiler, 2007; Murphy \& Rieke, 2008).

The distribution of the relative maintained values from WT tOFF GCs shows that $51 \%(n=25)$ had a PSS. A similar proportion of $\operatorname{TrpV1} 1 \%$ tOFF GC (42\%; Fishers exact test, $\mathrm{p}=0.42$ ) also had a PSS. However, the distribution of the $\operatorname{Trp} V 1 \%$ tOFF GCs relative maintained rates were positively skewed compared to WT and the mean ( $0.90 \mathrm{sp} / \mathrm{sec}$ above spontaneous activity) was significantly larger than WT (0.1 sp/sec below spontaneous activity; Fig. 3.8bii; $\mathrm{p}=0.001$ ). The latter result is consistent with the longer duration of response in $21 \%$ of $\operatorname{TrpV1} 1 \%$ tOFF GCs. As mentioned in that section, I cannot determine whether TRPV1 suppresses the maintained response and shortens duration in a small population of tOFF GCs, or enhances the maintained response and prolongs the duration in a population of SOFF GCs. Either way, the processes shaping the maintained response occurred after the peak, which was not affected by the absence of TRPV1. TRPV1 likely regulates feedback or feedforward inhibition to OFF BCs or GCs. The mechanisms shaping the GC maintained response are not fully understood. There is evidence suggesting that different types of OFF CBCs release glutamate in transient or sustained manners depending axon stratification centrally or proximally in the IPL respectively (Euler et al., 2015). Also, ACs modulate the temporal properties of OFF GCs directly. In order to affect the maintained response and not the peak, the kinetics of inhibitory receptors TRPV1 is affecting must be slowly activating. The following circuits are likely candidates: 
1. OFF CBCs receive GABAergic and, to a larger extent, glycinergic light-evoked inhibition. Prolonged inhibitory input which affects the prolonged release of glutamate is shaped by both glycine receptor and $\mathrm{GABA}_{c}$ receptor mediated input (Eggers \& Lukasiewicz, 2011). However, glycinergic input decreases both the transient and maintained release of glutamate from the OFF CBC (Ivanova et al., 2006). Therefore, TRPV1 may be regulating $\mathrm{GABA}_{c}$ inhibition to OFF CBCs. While OFF CBCs have slow $\mathrm{GABA}_{c}$ mediated feedback inhibition, $\mathrm{GABA}_{c}$ did not affect the maintained response of tOFF or sOFF GCs sampled in optic nerve recordings (Sagdullaev et al., 2006). If TRPV1 were regulating $\mathrm{GABA}_{c}$ mediated input, the maintained rates of OFF GCs in the optic nerve recordings should be similar in WT and $\operatorname{TrpV1} \%$.

2. Glycinergic input to the OFF GC or BC enhances the peak and maintained spiking responses of OFF GCs, likely through cross-over inhibition (Nobles et al., 2012). TRPV1 may enhance glycinergic input to a population of OFF BCs or GCs. Alternatively, TRPV1 may regulate serial inhibition of the ACs which regulate OFF GC spiking activity.

3. TRPV1 may be in OFF GCs, or affect properties of the OFF GC directly, which contribute to the spontaneous activity. My data from the next chapter shows TRPV1 is expressed in a subset of OFF GCs.

I made similar comparisons of the amplitudes (Fig. 3.9ai) and relative maintained rates (Fig. 3.9ai) in WT and TrpV1\% ON GCs. The distributions and 

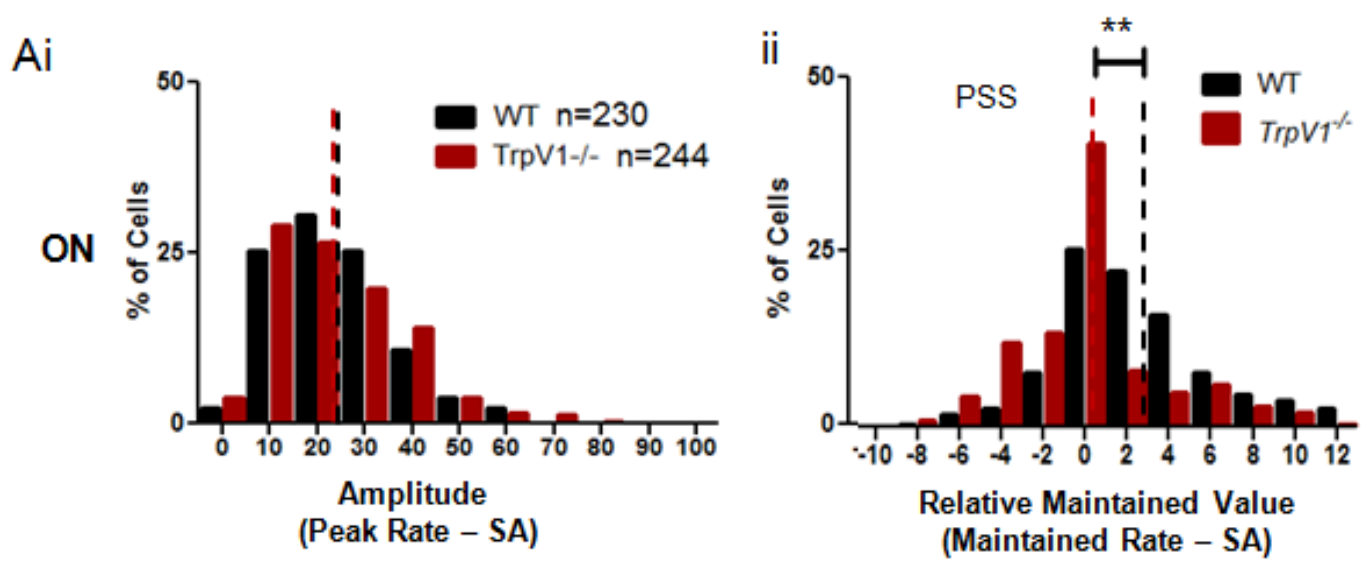

Figure 3.9 Maintained response rates were lower in $\operatorname{TrpV1}{ }^{-/}$ON GCs compared to WT. (Ai) I compared the peak amplitude (i) and maintained rates (ii; corrected for spontaneous activity) in WT and TrpV1- ON GCs. Amplitudes (Ai) were similar between WT $(23.9 \pm 0.8)$ TrpV1 1 ( $23.5 \pm 0.9)$ ON GCs $(p=0.43)$. (Aii) Relative maintained values were larger in $\bar{W} T$ $(2.5 \pm 0.3)$ compared to $\operatorname{TrpV1} 1^{-1}(0.2 \pm 0.3)$ ON GCs $(p<0.0001)$. TRPV1 does not contribute to the processes shaping the peak of the response, but regulates the maintained portion and duration of response of ON GCs.

means WT and TrpV1- ON GC amplitudes were similar (Fig. 3.9ai; $\mathrm{p}=0.43$ ).

From this I concluded that TRPV1 does not contribute to the peak response of ON GCs. The majority of WT ON GCs had sustained responses and positive relative maintained values with a mean of 2.5 (Fig. 3.9aii). Only $12 \%$ of WT ON GCs $(n=27)$ had a PSS. In contrast, significantly more TrpV1/ ON GC had a PSS than WT (Fig. 3.9aii; 32\%; Fishers exact test, $p<0.0001$ ). The amplitude of the PSS was not compared because the value is relative to spontaneous activity, which is significantly lower in TrpV1- ON GCs compared to WT. The majority of TrpV1\% ON GCs were transient and had relative maintained values less than or equal to zero. The mean $\operatorname{TrpV} 1 \%$ tON GCs relative maintained value (0.16) was lower than WT (Fig. 3.9aii; $p<0.0001$ ). From this, I conclude that TRPV1 does not 
contribute to the peak response, but enhances the maintained response of ON

GCs.

The maintained responses in ON GCs is determined by glutamate release
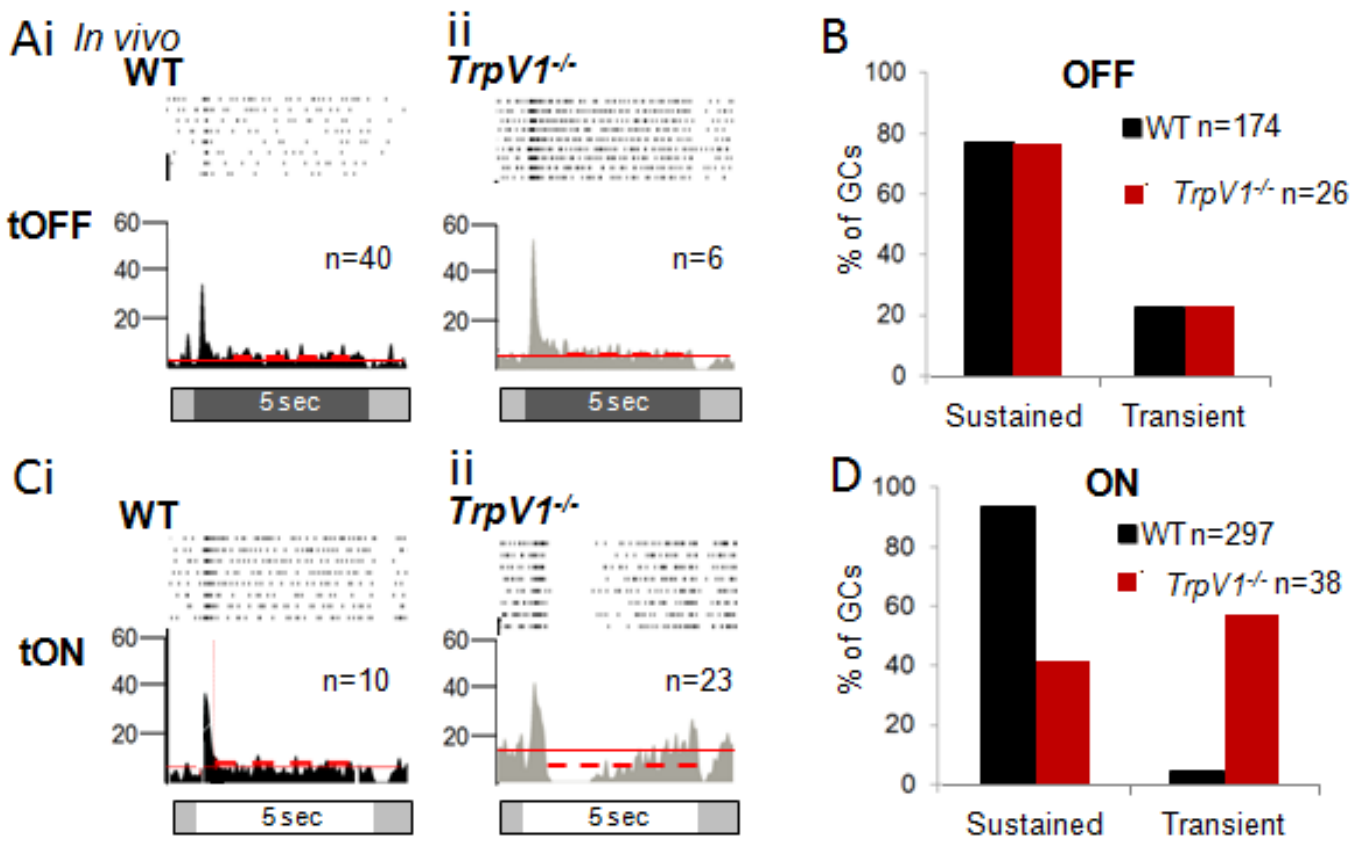

Figure 3.10 TrpV1/- ON GC were more transient than WT in vivo. (A) Representative PSTHs for WT (i) and $\operatorname{TrpV1} 1$ (ii) tOFF (A) and tON (B) GCs in response to a spot whose diameter matched the receptive field center are shown. Background (mean) luminance was 4.0 log $\mathrm{R}^{*} / \mathrm{rod} / \mathrm{sec}$. WT (Ai) and TrpV1\% (Aii) tOFF GCs had a transient peak response to a dark spot (3.0 $\log R^{*} / \mathrm{rod} / \mathrm{sec}$ ) which returned to spontaneous rates within 1.7s from stimulus onset. (B) The majority of WT OFF GCs (79\%) sampled in vivo were sustained. This is different than in vitro where the proportions of WT SOFF and tOFF GCs were approximately equal. Consistent with in vitro results, TrpV1/- had similar proportions of sOFF and tOFF GCs as WT (Fisher's exact test, $\mathrm{p}=0.89$ ). WT (Ci) and TrpV1/ tON (Cii) GCs had a transient peak response which returned to spontaneous rates ( $\mathrm{Ci}$; solid line) within 1.7s from stimulus onset in response to a light spot (4.7 $\log R^{\star} / \mathrm{rod} / \mathrm{sec}$ ) whose diameter matched the receptive field center. WT tON GCs continued to spike at spontaneous rates for the duration of the stimulation ( $\mathrm{Ci}$; dotted line). After the transient peak, the mean $\operatorname{TrpV1} 1^{-1} \mathrm{tON}$ GC spike rate was lower than spontaneous for 1 to $3 \mathrm{~s}$, then returned to spontaneous levels before stimulus offset (Cii). WT and TrpV1\% sON and sOFF GCs had a transient peak followed by a maintained response with spike rates greater than spontaneous levels for the entire duration of the stimulation. (D) Almost all of WT ON GCs (97\%, $n=287)$ sampled in vivo were sustained. Consistent with in vitro results, TrpV1\% ON GCs were more transient $(60 \%, n=23)$ than WT $(3 \%, n=10$; Fisher's exact test, $\mathrm{p}<0.0001)$. TRPV1 prolongs the duration of ON GCS sampled in vivo.

from ON BCs and shaped by inhibition. In the absence of TRPV1, inhibition to 
the ON CBC or ON GC is increased. The suppression of the response occurs after the transient peak, suggesting TRPV1 is likely regulating feedback or feedfoward inhibition. Also, the kinetics of the inhibitory receptors must be slow. TRPV1 may be acting in the following ways:

1. GABA $A_{c}$ mediated feedback inhibition of ON BCs decreases the maintained response of ON GCs (Sagdullaev et al., 2006; Eggers and Lukasiewicz, 2011). TRPV1 may inhibit the GABAergic AC providing the feedback inhibition to the ON BC. In the absence of TRPV1, the feedback AC is disinhibited and releases more GABA to $\mathrm{GABA}_{c}$ receptors in ON BCs (Nobles et al., 2012).

2. Glycinergic serial inhibition enhances the peak and maintained rates of $O N$ GCs in optic nerve recordings (Nobles et al., 2012). TRPV1 may enhance the tonic release of glycine from ACs which provide serial inhibition to GABAergic ACs.

3. TRPV1 in ON GC is regulating the spiking activity there. For this to hold true, TRPV1 would have to be in nearly all ON GCs. My data (investigated in the next chapter) does not support this hypothesis. TRPV1 resides in a small subset of ON GCs.

\subsection{TRPV1 activity prolonged duration of responses in the RF center of ON} GCs in vivo

To determine if TRPV1 contributes Sometimes I used a $5 \mathrm{~s}$ stimulus, similar to the in vivo recording (WT $\mathrm{n}=10 \& \operatorname{TrpV1} 1-\mathrm{n}=59$ ) of the stimulus (Nobles et al. 2012; Fig. 3.10a \& c). The intermediate durations ( $\geq 2<5$ s) observed in 
populations of OFF GCs in vitro were not observed in vivo. The majority of WT OFF GCs sampled in vivo had sustained responses (Fig. 3.10b). There were similar proportions of $\operatorname{TrpV1}^{1 /}$ sOFF or tOFF GCs compared to WT (Fig. 3.10b; Fisher's exact test, $\mathrm{p}=0.89$ ). Similar to in vitro results, there was a larger
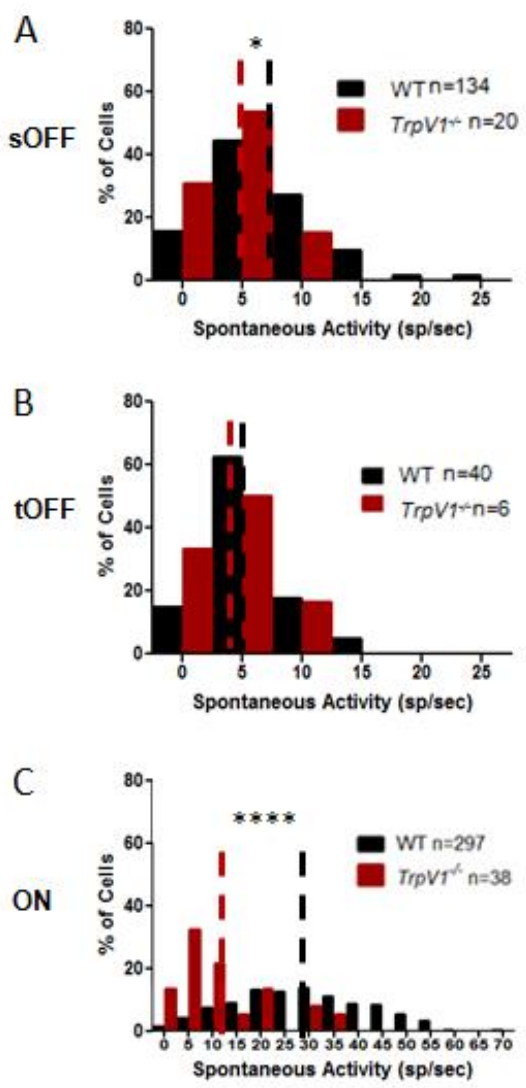

Figure 3.11 WT sustained GC spontaneous activity was greater than TrpV1\% in vivo (A) Consistent with in vitro results, spontaneous rates to a light background (4 log $\left.\mathrm{R}^{\star} / \mathrm{rod} / \mathrm{sec}\right)$ were greater in WT sOFF GCs $(7.1 \pm 0.4 \mathrm{sp} / \mathrm{sec})$ than $\operatorname{TrpV1}^{-1} \mathrm{sOFF}$ GCs $(4.8 \pm 0.6 \mathrm{sp} / \mathrm{sec} ; \mathrm{p}=0.03)$. (B) Spontaneous rates were similar between WT tOFF GCs $(5.8+0.6 \mathrm{sp} / \mathrm{sec})$ and $\operatorname{TrpV} 1^{-1-}$ tOFF GCs $(4.6 \pm 1.3 \mathrm{sp} / \mathrm{sec}$; $\mathrm{p}=0.52)$. (C) Spontaneous rates were greater in WT ON GCs $(29.8 \pm 0.8 \mathrm{sp} / \mathrm{sec})$ than $\operatorname{TrpV} 1^{-1}$ sON GCs $(12.3 \pm 2.3 \mathrm{sp} / \mathrm{sec}$; $\mathrm{p}<0.0001)$.

proportion of TrpV1\% tON GCs than WT (Fig. 3.10d; Fisher's exact test p<0.0001). Nearly all (287 out of 297) ON GCs sampled in the WT optic nerve recordings were sustained. In contrast, $60 \%$ of $\operatorname{TrpV} 1 \% \mathrm{ON}$ GCs were transient with response durations ranging from 0.3 to $1.7 \mathrm{~s}$. The larger proportion of TrpV11- tON GCs than WT in vivo is consistent with the idea that the absence of TRPV1 
$\mathrm{Ai}$

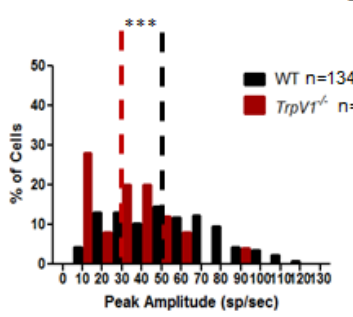

$\mathrm{Bi}$

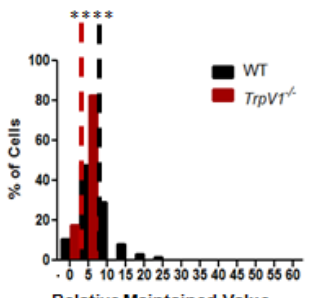

C

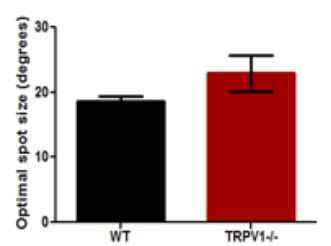

sOFF

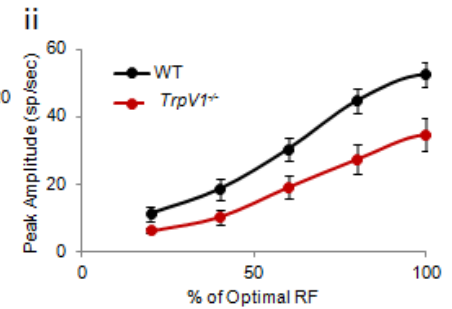

ii

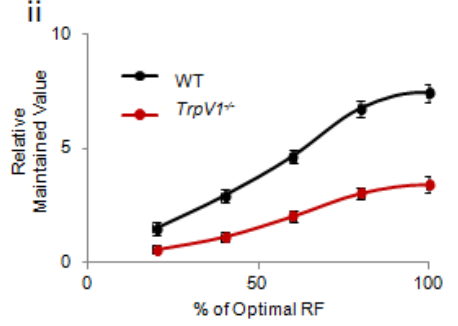

Figure 3.12 $\mathrm{TrpV1}^{-/}$sOFF GCs peak and maintained firing rates were lower than WT (Ai) Amplitudes were greater in WT sOFF GCs $(52.5 \pm 2.2 \mathrm{sp} / \mathrm{sec})$ than TrpV1\% sOFF $(31.9 \pm 4.0 \mathrm{sp} / \mathrm{sec})$ to spots whose diameter matched the RF center (Aii) WT sOFF amplitudes showed center summation; amplitudes increased with spot diameter up to the spot which matched the RF center. TrpV1\%sOFF amplitudes also had center summation and were lower than WT to all spots which stimulated the RF center $(p<0.0001)$. (Bi) Spontaneous activity was subtracted from the maintained response rate $(0.4$ to $2 \mathrm{~s})$ to obtain the relative maintained value. The relative maintained value was larger in WT sOFF GCs $(7.4 \pm 0.4 \mathrm{sp} / \mathrm{sec})$ than TrpV1/ $(3.4 \pm 0.3$ $\mathrm{sp} / \mathrm{sec} ; \mathrm{p}<0.0001)$ to a dark spot that matched the $\mathrm{RF}$ center. (Bii) WT sOFF relative maintained values showed center summation and $\operatorname{TrpV}^{1 /-}$ sOFF relative maintained values also had center summation and were lower than WT to all spots that stimulated the RF center $(p<0.0001)$. (C) The mean peak RF center diameter was similar between WT $(18.6+0.7$ degrees of visual angle) and TrpV1 $1-(22.9 \pm 2.8$ degrees visual angle; $p=0.088$ ) sOFF GCs.

alters the sustained

response in a class of

sON GC to a transient

response. This population

comprised $\sim 37 \%$ of $\mathrm{ON}$

GCs sampled in vitro and

$\sim 55 \%$ of ON GCs sampled

in vivo. Thus, TRPV1

enhanced the maintained

portion of the response

and subsequent duration

in the majority of ON GCs

sampled in vivo. The

aberrant transient

response was evoked by

stimuli matching the RF

center (in vivo) and full-

field stimuli which evoked

more of the inhibitory

surround (in vitro). Thus,

TRPV1 modulated center response properties enhanced the maintained

response in ON GCs. 


\subsection{TRPV1modulated excitatory properties of sustained OFF and ON GC}

receptive field centers in vivo

Spontaneous activity of WT sOFF and tOFF GCs and TrpV1\% sOFF and tOFF GCs was similar. TrpV1/- sOFF spontaneous activity $(4.8 \pm 0.6 \mathrm{sp} / \mathrm{sec})$ was lower than WT (Fig. 3.11a; $\mathrm{p}=0.03)$. WT $(5.8 \pm 0.6 \mathrm{sp} / \mathrm{sec})$ and TrpV1\% tOFF spontaneous activity $(4.6 \pm 1.3 \mathrm{sp} / \mathrm{sec})$ was similar (Fig. 3.11b; $\mathrm{p}=0.52)$. TrpV1ON spontaneous activity $(12.3 \pm 2.3 \mathrm{sp} / \mathrm{sec})$ was lower than WT (Fig. 3.11c; $29.8 \pm 0.8 \mathrm{sp} / \mathrm{sec} ; \mathrm{p}<0.0001)$. Consistent with in vitro results, TRPV1 contributes to processes driving spontaneous activity in sustained OFF and ON GCs.

WT sOFF GCs had greater amplitudes than $\operatorname{TrpV1}{ }^{-1}(\mathrm{p}=0.003)$ to spots whose diameter matched the RF center. This was consistent across all smaller spots as well (Fig. 3.12ai \& aii). Both WT and TrpV1\% sOFF GCs showed center summation; amplitudes increased with spot diameter up to the spot which matched the receptive field center. This differed from my in vitro results where I did not find a difference in sOFF amplitude between genotypes using a full-field photopic stimulation. To determine if this resulted from the difference in the stimulus (full-field vs spot confined to the RF center), I compared the WT sOFF amplitude to the largest spot. Again, WT sOFF amplitude was larger than TrpV1\% $(p=0.01$; data not shown). Thus the difference was more likely to represent the differences in the cell classes that comprise sOFF cells in vivo and in vitro. From this I concluded that TRPV1 activity enhances the excitatory peak amplitude to RF center stimulation in the population on sOFF GCs sampled in vivo. In the absence of TRPV1, this enhancement could take the form of 


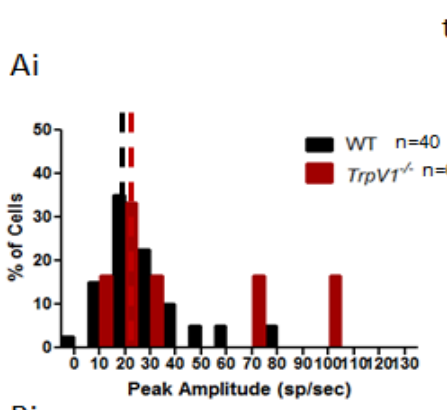

$\mathrm{Bi}$

tOFF

ii
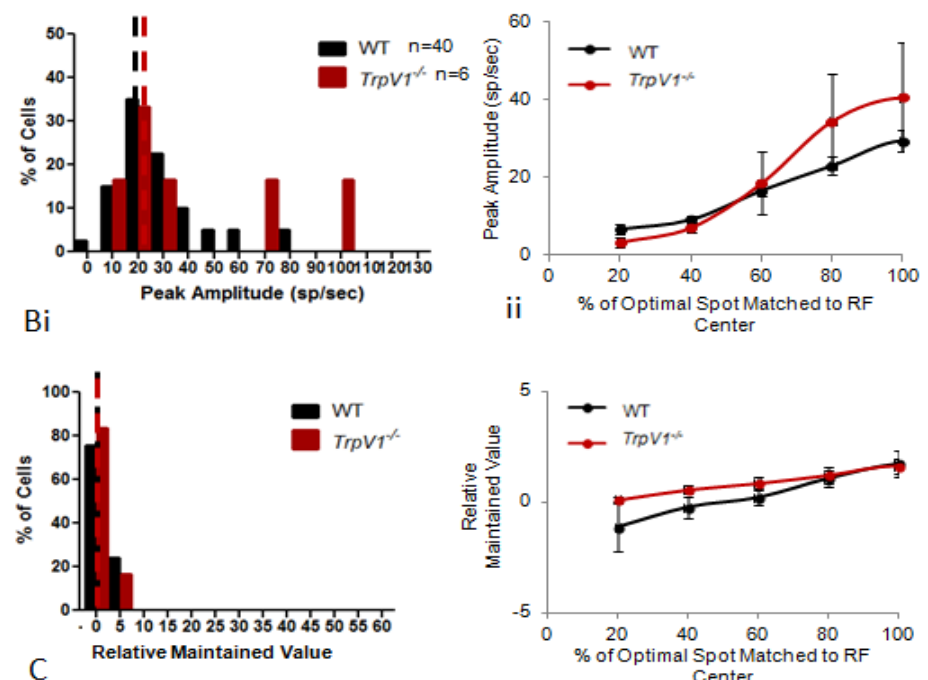

C

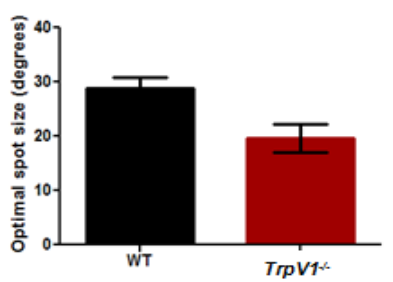

Figure 3.13 $\mathrm{TrpV1}^{-/}$tOFF GC peak and maintained firing rates were similar to WT (Ai) Amplitudes were similar in WT $(29.4 \pm 2.7$ $\mathrm{sp} / \mathrm{sec})$ and TrpV1-/ tOFF GCs $(40.5+14.0 \mathrm{sp} / \mathrm{sec} ; \mathrm{p}=0.71)$ to spots whose diameter matched the RF center (Aii) WT tOFF amplitudes showed center summation. TrpV1/-tOFF amplitudes had center summation and were similar with WT to all spots which stimulated the RF center ( $p=0.85)$. (Bi) Spontaneous activity was subtracted from the maintained response rate $(0.4$ to $2 \mathrm{~s})$ to obtain the relative maintained value. The mean relative maintained value was similar in WT $(1.6+0.3 \mathrm{sp} / \mathrm{sec})$ and TrpV1\% tOFF GCs $(1.6+0.3 \mathrm{sp} / \mathrm{sec} ; \mathrm{p}=0.93)$ to a dark spot that matched the RF center. (Bii) WT tOFF relative maintained values were similar to all spots which stimulated the RF center $(p=0.84)$ and TrpV1- tOFF relative maintained values were similar to WT $(p=0.73)$. (C) Mean peak RF diameters were similar between WT (28.9 \pm 1.8 degrees of visual angle) and $\operatorname{TrpV} 1^{-1-}(19.7 \pm 2.6$ degrees visual angle; $p=0.058$ ) tOFF GCs. increased inhibition on

the OFF GC or

decreased glutamate

release from the OFF

BCs. The mean RF

center diameter of

TrpV1\% sOFF GCs

was similar to WT

(Fig. 3.11c; $p=0.088)$;

TRPV1 did not

contribute to RF

spatial tuning of the

peak response. This

suggests both center

and surround

response properties

contributing to the

peak were affected

equally. This is

indicative of increased

tonic suppression of

the sOFF GC sampled in vivo. TRPV1 may regulate disinhibition to the OFF GC.

Otherwise TRPV1 by be affecting the spiking activity in the OFF GC directly. 
The mean relative maintained value in WT sOFF GCs to spots whose diameter matched the RF center was $7.4 \pm 0.4 \mathrm{sp} / \mathrm{sec}$ and relative maintained value showed center summation, like the amplitudes. WT sOFF GCs mean relative maintained values in response to optimal $\mathrm{RF}$ stimulation was greater than TrpV1/ sOFF GCs $(3.4 \pm 0.3 \mathrm{sp} / \mathrm{sec} ; \mathrm{p}<0.0001)$. This was consistent across all spots that stimulated the RF center (Fig. 3.12bi \& bii). TrpV1\% sOFF relative maintained values had center summation as well. From this I concluded that TRPV1 activity enhances the transient and maintained center response in the population of sOFF GCs sampled in vivo. In the absence of TRPV1, the shapes of the amplitude and maintained ARFs were similar to WT (data not shown). TRPV1 may act to inhibit local inhibition to sOFF GCs or their OFF BC synaptic partners under light adapted conditions.

Amplitudes were similar between WT $(29.3 \pm 2.7 \mathrm{sp} / \mathrm{sec})$ and TrpV1\% tOFF GCs $(40.5+14.0 \mathrm{sp} / \mathrm{sec} ; \mathrm{p}=0.71)$ to spots whose diameter match the RF center (Fig. 3.13ai). This was consistent across all smaller spots as well (Fig. 3.13ai \& aii). Both WT and TrpV1- tOFF GC amplitudes showed center summation $(\mathrm{p}<0.0001)$. The mean RF center diameter of TrpV1\% tOFF GCs was similar to WT (Fig. 3.13c; $p=0.06$ ). From this I conclude TRPV1 did not contribute to the center response properties that shaped the peak amplitudes of tOFF GCs sampled in vivo.

Relative maintained values of WT tOFF GCs were constant regardless of spot diameter (Fig. 3.13bii; $p=0.42$ ). TrpV1 1 tOFF GC Relative maintained values were similar to WT with RF center stimulation ( $p=0.93)$ and this was 

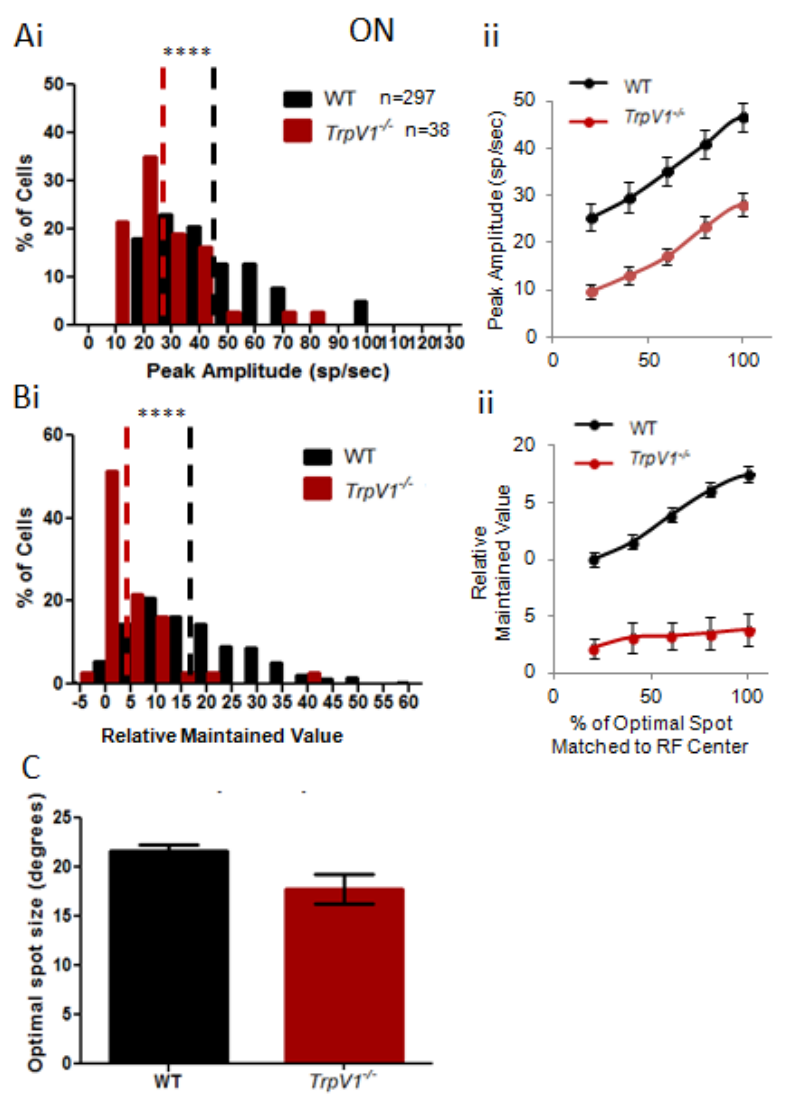

Figure 3.14 $\operatorname{TrpV1}^{-/}$ON GC peak and maintained firing rates were lower than WT (Ai) Amplitudes were greater in WT ON GCs $(47.9 \pm 1.2 \mathrm{sp} / \mathrm{sec})$ than TrpV1\% ON GCs $(26.9+2.7$; $\mathrm{p}<0.0001$ ) to spots whose diameter matched the RF center (Aii) WT ON amplitudes showed center summation. TrpV1\% ON amplitudes also had center summation $(p<0.0001)$ and were lower than WT to all spots which stimulated the RF center $(p<0.0001)$. (Bi) Almost all WT ON GCs were sustained and therefore had positive relative maintained values. The mean relative maintained value was larger in WT ON GCs $(17.2 \pm 0.7$ $\mathrm{sp} / \mathrm{sec})$ than $\operatorname{TrpV1} \%(4.7 \pm 1.3 \mathrm{sp} / \mathrm{sec} ; \mathrm{p}<0.0001)$ to a light spot that matched the RF center. This was consistent with the large proportion (55\%) of tON GCs in TrpV1\% (Bii) WT ON relative maintained values showed center summation $(p<0.0001)$. In contrast, TrpV1 1 ON relative maintained values were constant regardless of spot size and were lower than WT to all spots which stimulated the RF center $(p<0.0001)$. (C) RF diameters were similar between WT $(21.6 \pm 0.7$ degrees of visual angle) and TrpV1\% (17.8 \pm 1.5 degrees of visual angle; $p=0.052)$ ON GCs. consistent across spot

sizes within the $R F$

center (Fig. 3.13bi \&

bii; $p=0.36)$. In

contrast, the

maintained rates and

response durations of

the TrpV1\% tOFF GCs

recorded in vitro were

larger than WT tOFF

GCs. This difference

between my in vivo

and in vitro data was

due to the contribution

from a population

$(20 \%)$ of $\operatorname{TrpV1}{ }^{-/}$tOFF

GCs with long

response durations

(2>5s), which were

not observed in either

genotype in vivo. I

conclude that TRPV1 does not contribute to the maintained response in the population of tOFF GCs sampled in vivo. 
WT ON GCs had greater amplitudes $(47.9 \pm 1.2 \mathrm{sp} / \mathrm{sec})$ than $\operatorname{TrpV1}{ }^{-1}$ (Fig. 3.14ai; $26.9+2.7, p<0.0001$ ) to spots whose diameter matched the RF. This was consistent across all spots smaller than the RF center (Fig. 3.14aii). WT and TrpV1\% ON GCs showed center summation (Fig. 3.14aii). These results were different than the in vitro results; there was no difference in TrpV1\% ON peak amplitude compared to WT to photopic full-field stimuli. WT ON GC amplitude

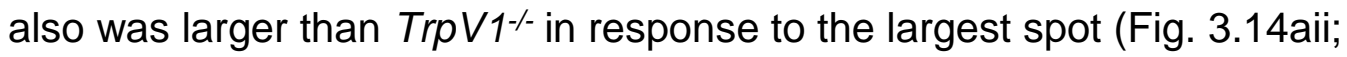
$p<0.0001$; data not shown), which evokes surround suppression. From this I concluded that TRPV1 enhances the peak response to center stimulation in the population of ON GCs sampled in vivo. The shape of the peak ARF was similar between genotypes (data not shown) and RF diameters were similar in WT and TrpV1- ON GCs (Fig. 3.14c; $\mathrm{p}=0.052$ ), which indicates TRPV1 modulates center and surround response properties that shaped the peak in similar proportions. TRPV1 may modulate local inhibition in the RF center to the ON GC or ON BCs (Nobles et al., 2012).

Almost all WT ON GCs recorded in vivo had sustained responses. The mean relative maintained value in response to a spot whose diameter matched the RF center was $17.2( \pm 0.7) \mathrm{sp} / \mathrm{sec}$ above spontaneous activity (Fig. 3.14bi). The relative rate in WT ON GCs showed center spatial summation; relative maintained values were lower to smaller spots (Fig. 3.14bii). The mean relative maintained value in response to optimal RF stimulation in $\operatorname{TrpV1\% }$ ON GCs $\left(4.7_{ \pm}\right.$ $1.3 \mathrm{sp} / \mathrm{sec}$ ) was lower than WT (Fig. 3.14bi; $\mathrm{p}<0.0001$ ). This was consistent 
across all spot sizes (Fig. 3.14bii; $p<0.0001$ ). Interestingly, the ARF of the TrpV1-

1- ON relative maintained values across spot size was flat rather than the inverted

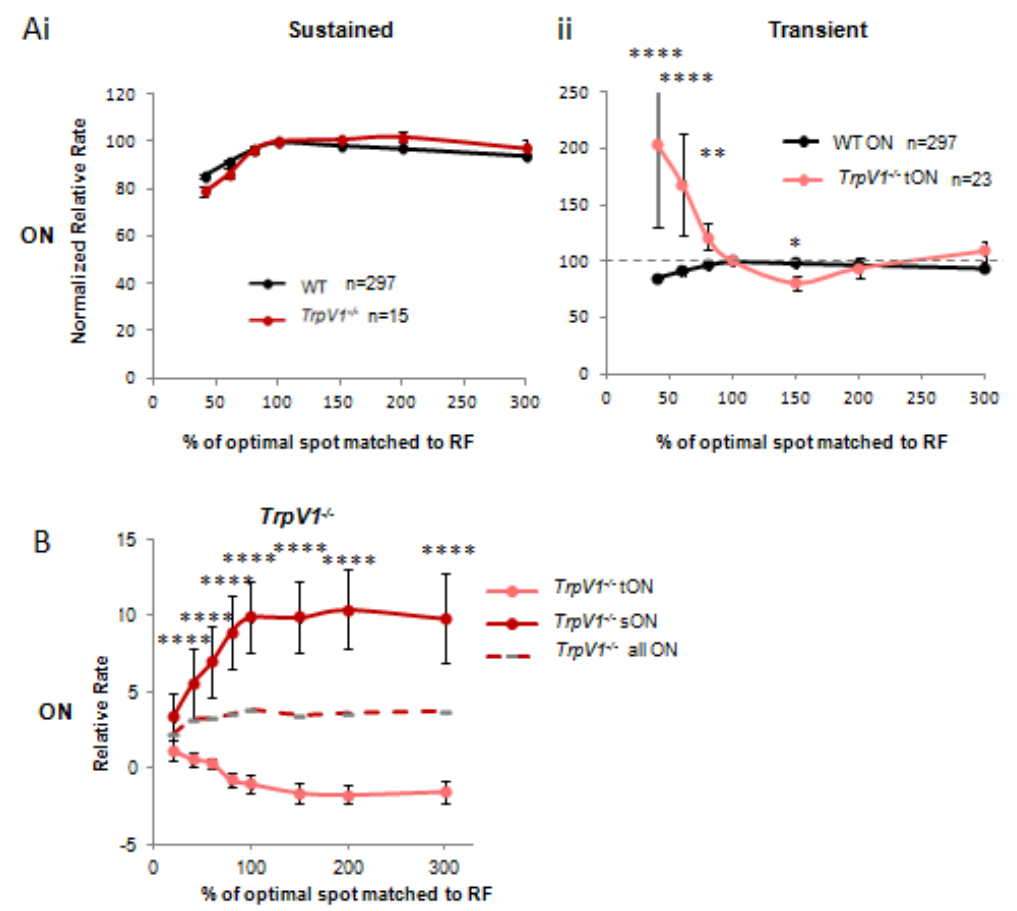

Figure 3.15 $\mathrm{TrpV1}^{-/}$tON and SON GC maintained firing rates had inverse ARFs (Ai) Relative maintained values were normalized to the value in response to a spot whose diameter matched the RF center and graphed as \% of relative maintained value as a function of \% of optimal spot matched to RF diameter. Like peak amplitudes, WT ON relative maintained values were spatially modulated $(p<0.0001)$; rates increased proportionally with spot diameter and were greatest to a spot whose diameter matched the RF center. Spots larger than the RF center evoked surround suppression of the relative maintained values. The percent change in relative rates in TrpV1/- sON GCs was similar to WT to all spot sizes $(\mathrm{p}=0.80)$. (Aii) TrpV1/- tON relative maintained values in the aberrant $\operatorname{TrpV} 1 / \mathrm{tON}$ GCs also were spatially modulated $(p<0.0001)$, however modulation was the inverse of the WT (and Trp V1/) sON GCs $(p<0.0001)$. Stars indicate difference from relative rate to spot matched to RF center $(100 \%)$. Spots whose diameters were smaller than the RF center evoked larger relative rates than spots matched to the RF center. Minimum relative maintained values, which represent the largest suppression of the maintained response, were evoked by spots $50 \%$ larger than the RF center. Relative maintained values to spots larger than that were similar to RF center rate, indicating local inhibition suppressed maintained responses more than surround inhibition. (B) Stars indicate difference between tON and sON relative rates. Relative maintained values for TrpV1/- sON and tON GCs were similar and positive (sustained) to spots whose diameters were $20 \%$ of the RF center. As spot size increased to RF center, $\operatorname{TrpV} 1 \% \mathrm{sON}$ relative maintained values increased and TrpV1\% tON relative maintained values decreased. TrpV1 tON relative maintained value was negative, indicating PSS of the response below spontaneous activity, to spots whose diameters were $>80 \%$ of the RF center. The ARF of the tON and sON GCs combined lacked spatial modulation. 
U shape in the WT ON GCs, indicating that among all TrpV1- ON GCs, the maintained rates did not have center summation (Fig. 3.14bii). Taken together, my results suggested TRPV1 enhances spontaneous and light evoked spiking activity in the majority of ON GCs sampled in vivo.

\subsection{TRPV1 likely enhances serial inhibition of $\mathrm{GABA}_{c}$ receptor mediated} responses in ON BCs

To investigate the absence of spatial summation in the relative rates of TrpV1\% ON GCs, I normalized WT and TrpV1 ${ }^{-}$ON GC relative maintained values to the value in response to a spot whose diameter matches the receptive field center. I compared the normalized values in $\operatorname{TrpV} 1 \% \mathrm{sON}$ and tON GCs individually to WT ON GCs. TrpV1\% sON GCs had a similar ARF to WT; the relative maintained values had similar spatial properties (Fig. 3.15ai). Relative maintained values in the aberrant $\operatorname{Trp} V 1 \% \mathrm{tON}$ GCs were spatially modulated (Fig. 3.15aii; $p<0.0001)$. However, the shape of the TrpV1\%tON area response function was opposite that of the of the WT ON GCs $(p<0.0001)$. Spots with diameters that were smaller than the RF center evoked larger relative maintained values than spots matched to the RF center (Fig. 3.15aii). Minimum relative rates, which represent the largest suppression of the maintained response, were evoked by spots $50 \%$ larger than the RF center. Relative maintained values to spots larger than that were similar to RF center rate, indicating local inhibition suppressed maintained responses. Surround 
suppression did not increase the total suppression of the relative maintained

value (Fig. 3.15aii). The combination of the inverted $U$ shaped ARF in the TrpV1/-
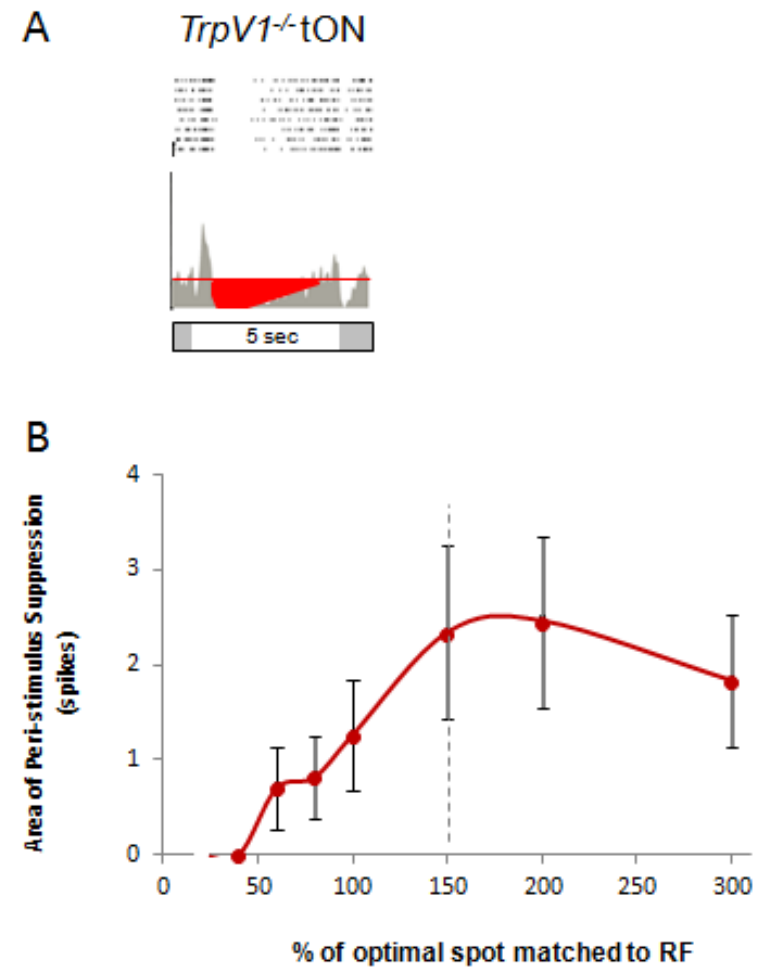

Figure 3.16 $\operatorname{TrpV1}^{-/}$tON GC peristimulus suppression had center summation and surround suppression. (A) In the majority of TrpV1- tON GCs (80\%) there was a transient increase in the spiking rate in response to light spots whose diameter matched the RF center. One sec after stimulus onset, the spike rate was suppressed below spontaneous activity. The peri-stimulus suppression continued for one to three seconds, then returned to spontaneous rates before the offset of the five sec stimulus. The area of the peri-stimulus suppression, shaded red, was quantified as a function of spot diameter in relation to the RF center diameter (B) There was peri-stimulus suppression to spots whose diameters were $\geq 60 \%$ of the RF center. Suppression increased proportionally with spot diameter. Spots whose was $50 \geq 100 \%$ larger than RF center evoked the maximum suppression. Suppression area decreased to larger spots. Thus, the ACs modulating the PSS receive surround inhibition.

sON GCs and the $U$ shaped in the Trp $1 \%$ tON formed a flat function when all ON GCs were compared together (Fig. 3.15b). The suppression in the response to spots slightly larger than optimal suggests that in the absence of TRPV1 there is an increase in local inhibition either onto a population of sON GCs or the presynaptic ON CBCs. Furthermore, the inhibition shaping the PSS of the maintained response had center surround spatial properties. I investigated this further by assessing the spatial modulation of the PSS. 

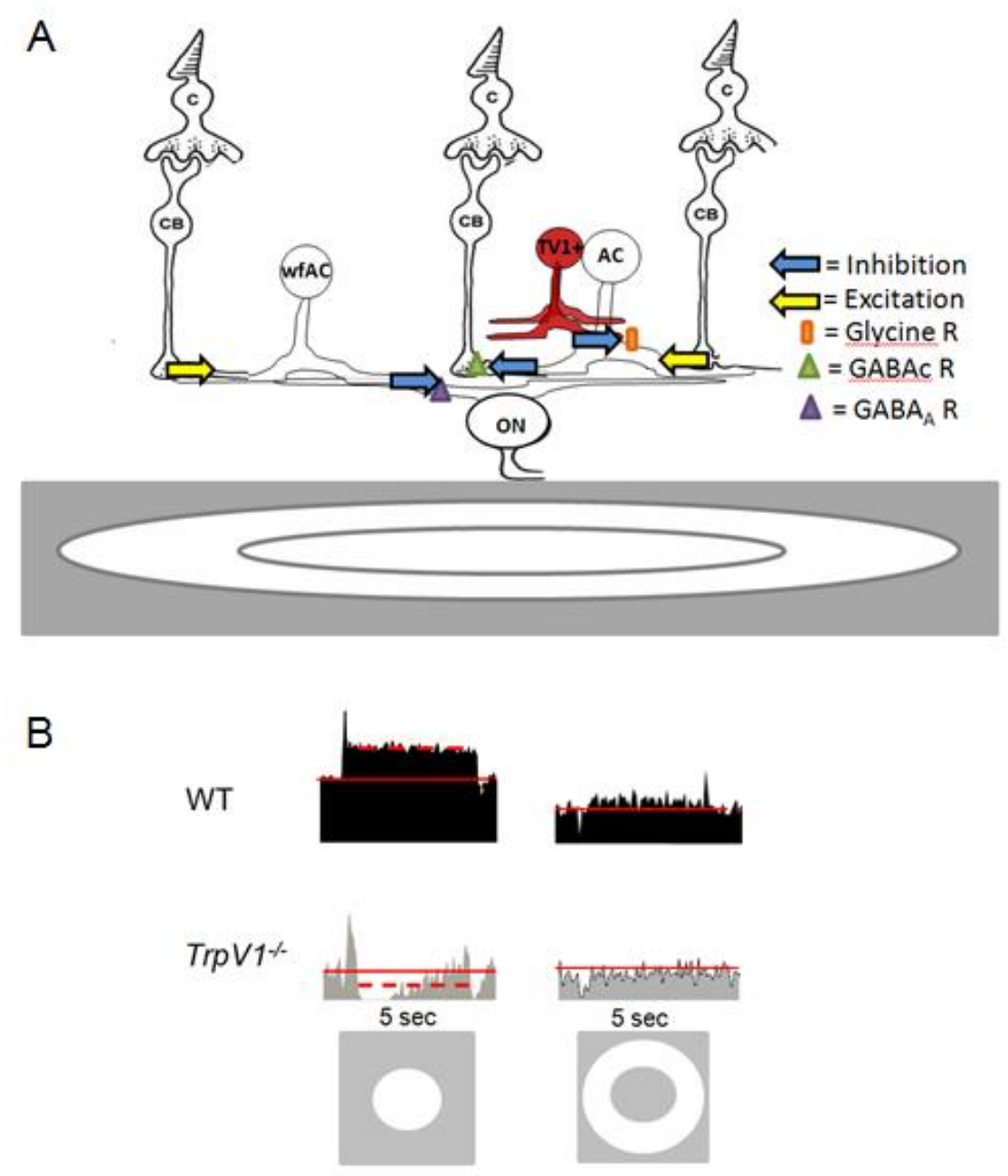

Figure 3.17 Possible explanation for the peri-stimulus suppression of maintained rates in TrpV1/ ON GC (A) In WT, ON CBCs, which were presynaptic to ON GCs, depolarized to a light spot in the ON RF center. The depolarization resulted in the release of glutamate to the $\mathrm{ON} \mathrm{GC}$ and to a GABAergic $A C$ which releases GABA on the $\mathrm{GABA}_{c}$ receptor, which is located in the $\mathrm{ON} B C$. The GABAergic $A C$ likely provides surround suppression to the $\mathrm{ON} B C$. A TRPV1 positive AC (likely glycinergic) tonically inhibits the GABAergic $A C$ in light adapted conditions. In the absence of TRPV1, the GABAergic AC is disinhibited and GABAc mediated inhibition to the ON CBC is increased. The ultimate result would be the suppression of the maintained release of glutamate and subsequent maintained spiking response in ON GCs to center stimulation (B). A different GABAergic AC provides surround suppression to $G_{A B A}$ receptors on the ON GC. That $A C$ would likely have transient glutamate release, resulting in transient surround suppression (B), which does not add to the suppression of the maintained response. With this prediction, the TRPV1 ON GCs which have transient center responses should have transient surround suppression to annular stimuli whose inner diameter matches the RF center. 
In the majority of $\operatorname{TrpV1} 1 \%$ tON GCs (80\%), there was PSS of the maintained response, which began directly after the transient peak with one to three sec duration (Fig. 3.16a). To investigate this aberrant PSS in TrpV1\% ON GCs, I calculated the total area of the TrpV1\% tON PSS in response to each spot size. I graphed the PSS area as a function of the percent of spot size relative to the spot whose diameter matched the RF center (Fig. 3.16b). I found little or no suppression in responses to spots $\leq 40 \%$ of the optimal RF. Suppression appeared when spots were $\geq 60 \%$ of the optimal RF and suppression increased with spot size. Spots that were 1.5 to 2 times larger than the optimal RF produced the largest suppression (Fig. 3.16b). Thus, the aberrant PSS which is generated in the absence of TRPV1 mediated input showed center summation and surround suppression, indicating the ACs mediating the local inhibition receive surround inhibition from other (likely wide-field) ACs. A circuit like this has been identified in the mouse. Medium field GABAergic ACs release GABA onto $\mathrm{GABA}_{c}$ receptors located in $\mathrm{ON} B C$ terminals. In this way, ACs enhance the surround suppression of the ON BC to stimuli larger than the BC receptive field (Eggers \& Lukasiewicz, 2010; Eggers et al., 2013; Moore-Dotson et al., 2015). Spiking GABAergic and glycinergic ACs serially inhibit the AC providing BC surround suppression. In the absence of TRPV1, surround suppression to the ON CBC is increased and glutamate release is decreased (Fig. 3.17a). Thus, in the $\operatorname{TrpV} 1 \%$, spot stimuli that are localized to the RF center of the BC $(25 \mu \mathrm{m}$; Eggers \& Lukasiewicz, 2010) would produce sustained release of glutamate from ON CBCs. Spots whose diameter activates the surround of the ON BC, but are 
still smaller than the GC RF center, would suppress the maintained release of glutamate from the ON BC, and the maintained spiking response of the ON GC.

Surround suppression in the IPL arises from lateral feed-forward inhibition to BCs and GCs (Cook \& McReynolds, 1998; Flores-Herr et al., 2001). Since activation of the GC surround does not enhance the PSS, a different GABAergic AC likely provides surround suppression to the ON GC. Surround suppression in ON GCs which have tON center responses in the absence of TRPV1 (Fig. 3.17b). This suggests the surround suppression coming into the ON GC directly was transient and therefore did not add to the peri-stimulus suppression, which lasted for two to four sec. If my hypothesis were true, the surround suppression to the $\operatorname{TrpV} 11$ tON GCs would have been transient and thus not enhance the peri-stimulus suppression of the maintained response (Fig. 3.17b). I used annular stimuli to isolate the surround suppression to ON GCs and compared the suppression properties in $\operatorname{TrpV1}{ }^{-/} \mathrm{tON}$ and sON GCs to WT (Fig. 3.18). A larger percent of WT ON GCs (92\%) had surround suppression than TrpV1\% ON GCs (Fig. 3.18a; 62\%, p=0.004), indicating TRPV1 enhanced surround suppression to ON GCs. TRPV1 activity may enhance glutamate release from ON BCs outside the RF center of the ON GC, or may act directly on the wide-field AC responsible for surround suppression of ON GCs. As mentioned, the majority of WT ON GCs had sustained responses to RF center stimulation. Among these GCs, approximately half of them (56\%) had transient surround suppression and the remainder had sustained surround suppression (Fig. 3.18bi). The majority of TrpV11- ON GCs had transient center responses (73\%). As predicted, the 

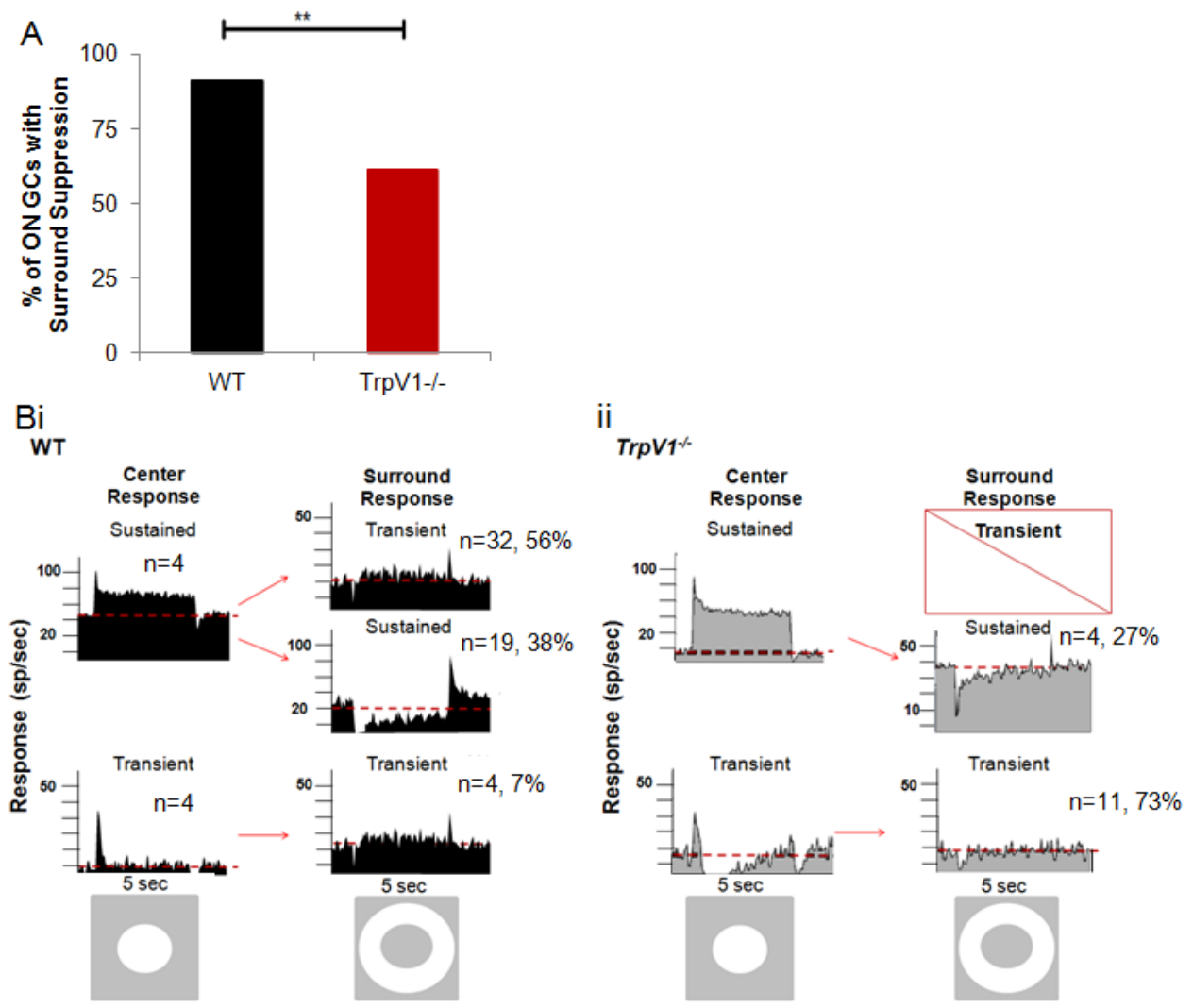

Figure 3.18 TRPV1 enhanced the maintained response in ON center GCs with transient surround suppression. (A) Annular stimuli were presented to ON GCs to evoke the surround suppression. A larger percent of WT ON GCs have surround suppression (92\%) than TrpV1ON GCs (62\%; Fisher's exact test, $p=0.004)$, indicating TRPV1 enhanced the surround suppression to sON GCs. (Bi) Among the WT ON GCs with surround suppression, $93 \%$ had sON center responses; $56 \%$ had sON center responses with transient surround suppression, while $38 \%$ had sON center, sustained surround responses. Four tON center GCs had transient surround suppression (7\%) (Bii) Among the TrpV1- ON GCs with surround suppression, the minority had sON center responses (27\%) and those four sON center GCs had sustained surround suppression. The surround suppression was transient in all TrpV1\% tON center GCs with suppression (73\%). Therefore, the population of TrpV1/- ON GCs with peri-stimulus suppression of the maintained response had transient surround suppression, consistent with my proposed model (Fig. 3.17)

surround suppression in all TrpV1/- tON GCs was transient (Fig. 3.18bii). TrpV1/-

sON GCs had sustained surround suppression if it was present (four out of nine).

While this suggested the center maintained response within a specific GC class

was enhanced by TRPV1 activity, further experiments would be necessary to 
confirm such a hypothesis. My attempts to locate such GCs among fluorescently labeled GC classes have been unsuccessful so far.

\section{Discussion}

\subsection{TRPV1 modulates signaling through several retinal pathways}

TRPV1 activity enhances spontaneous and light evoked GC spiking activity within several pathways. In the rod pathway, TRPV1 enhances the sensitivity of GCs to respond to threshold luminance. In the cone pathway, TRPV1 enhances the spontaneous and light evoked activity in ON and OFF GCs. Within the ON and OFF pathways, TRPV1 acts specifically, on the sustained temporal pathway. Within the sustained pathway, TRPV1 activity enhances the center response properties in sub-populations of SON and SOFF

GCs. All of this suggests TRPV1 acts to enhance the extraction of specific information from the visual world. The spiking activity of the GCs gives a general idea of the role of TRPV1 in shaping visual processing, which can be investigated further through the investigation of the specific circuits which shape the spiking activity. The first circuit to investigate would be one which shapes the very specific effects observed in TRPV1 sON and sOFF GCs. I will discuss such a circuit within the parallel pathways individually.

\subsection{TRPV1 likely enhances temporal signaling through the ON pathway} through amacrine cell mediated inhibition 
My results showed that TRPV1 enhanced the excitatory peak and maintained response to center stimulation in $55 \%$ of sON GCs sampled in vivo. In addition, TRPV1 modulated spatial properties of the maintained response in that population, but not the transient peak. TRPV1 enhanced the maintained response in approximately half of the sON GCs sampled in vivo as well, but did not alter the peak. Thus, within the ON cone pathway, TRPV1 is required to match the duration of the response to the duration of the stimulus in approximately half of the GCs which do that. Also the TRPV1 mediated circuit that regulates the temporal responses in these GCs is independent of the processes regulating the peak response to the majority of these GCs.

The processes modulating the duration of the maintained response is GCs is not fully understood but appears to be regulated by a combination of the duration of enhanced glutamate release from $\mathrm{BCs}$ and $\mathrm{AC}$ modulation of the signal. Recordings from CBCs in salamander and recently in mouse show a gradient of the duration of glutamate release related to stratification in the IPL (see Euler et al., 2015 for review). CBCs and GCs stratifying closer to the nuclear layers have more sustained responses while those in the center are more transient. This suggests the transient spiking in GCs comes from transient glutamate release from CBCs. The maintained response can also be modulated by ACs in the IPL. Specifically, a GABA ${ }_{c}$ mediated feedback loop to ON CBCs decreases the maintained activity in sON GCs (McCall et al., 2004). Because the peak ARF spatial profile is not altered by the removal of TRPV1, the disruption of the excitation/inhibition balance is occurring in the IPL, after the events forming 
the transient have passed. The most likely candidate for the decrease in the maintained rate is an increase in feedback inhibition to the ON CBC via the $\mathrm{GABA}_{c}$ receptor or an increase in GABAergic or glycinergic feedforward inhibition to the ON GC (Nobles et al., 2012). In this case, TRPV1 activity would regulate serial disinhibition of the feedback or feedforward ACs, as proposed in Figure 3.17. The expression of TRPV1 mainly in glycinergic ACs in the retina (see Chapter 4) indicates TRPV1 most likely modulates glycinergic inhibition (O'Brien et al., 2003; Manookin et al., 2008; van Wyk et al., 2009; Chen et al., 2010; Nobles et al., 2012; Zhang and McCall, 2014).

Feedback inhibition is ultimately mediated by GABA release onto the $\mathrm{GABA}_{A}$ and $\mathrm{GABA}_{c}$ receptors in ON CBCs (Zhang et al., 1997; Eggers et al., 2007). GABAA receptors have fast kinetics and $\mathrm{GABA}_{A}$ mediated inhibition can decrease the transient release of glutamate from $B C s$. $G A B A_{c}$ receptors have slower kinetics and modulate the prolonged release of glutamate (Eggers \& Lukasiewicz, 2011). Dong and Werblin (1998) showed that blocking GABA $c$ mediated feedback inhibition using the selective $G A B A_{c}$ receptor antagonist TPMPA enhanced the maintained response and increased the duration, but did not make transient cells sustained. Sagdullaev and colleagues (2006) also showed that the maintained response of sON GCs is enhanced in $\mathrm{GABA}_{c}$ knockout mice in vivo. Since all spiking activity in ON GCs is driven by glutamate from the ON BCs, if glutamate release is suppressed below the spontaneous release, then the spiking activity of the ON GC will also be suppressed below spontaneous. Maintained rates are suppressed below spontaneous activity in 
TrpV1\% ON GCs, TRPV1 activity likely enhances the maintained response in ON GCs by inhibiting the GABA $A_{c}$ mediated lateral inhibition to ON BCs (Fig. 3.17).

TRPV1 enhancement of the maintained response could be mediated through tonic serial inhibition. GABAergic amacrine cells mediate both wide field (Cook \& McReynolds, 1998; Flores-Herr et al., 2001) and local feedback inhibition (Dong \& Werblin, 1998; Freed et al., 2003) to BCs and GCs. GABAergic feedback to the RF center of rabbit local edge detector GCs is controlled by serial glycinergic inhibition (Russell and Werblin, 2010). Also salamander GC responses are mediated through serial inhibition (Zhang et al., 1997). Nobles and colleagues (2012) found GlyRa2 and GlyRa3 combined mediated tonic serial inhibitory input to ON GCs which enhances the peak and maintained responses of ON GCs in vivo. My in vivo results from the TrpV1- are similar to the GlyRa2 and GlyRa3 results from that publication, further supporting the idea that TRPV1 activity is mediating inhibition of local feedback and feedforward inhibitory circuits. If this were true, then TRPV1 activity promotes the tonic release of glycine from the ACs in which it is expressed onto ACs regulating the feedforward and feedback circuits.

\subsection{TRPV1 likely enhances spontaneous and evoked signaling through the} OFF pathway via amacrine cell mediated inhibition

Spontaneous activity was lower in TrpV1/ sOFF GCs compared to WT in vivo and in vitro. Spontaneous activity is internally generated in large OFF GCs (Margolis \& Detwiler, 2007) and modulated by excitatory and inhibitory neurotransmission. In the absence of cross-over inhibition from the ON pathway, 
OFF GCs depolarize and spontaneous spiking increases (Zaghloul et al., 2003). In the absence of TRPV1, OFF spontaneous activity is lower, suggesting that cross-over inhibition is intact and there may be increased inhibition or decreased excitation to the OFF GCs compared to WT. Nobles and colleagues (2012) report Glyra2 mediated inhibition enhances the spontaneous activity in OFF GCs. TRPV1 regulation of tonic glycinergic inhibition may be enhancing OFF spontaneous activity through these receptors.

Peak responses were not affected by the absence of TRPV1 in the population of sOFF GCs sampled in vitro, however the peak and maintained responses were lower in the population of $\operatorname{TrpV1} 1 \%$ sOFF GCs sampled in vivo. The shapes of the peak and maintained ARFs were similar to WT. Thus, in a population of sOFF GC which is not highly represented in MEA recordings, center and surround excitatory and inhibitory processes were equally affected by the lack of TRPV1. This TRPV1 mediated enhancement of spontaneous and light evoked spiking activity similarly is suggestive of a common source. In the absence of TRPV1 there is increased tonic inhibition to the OFF BCs or the postsynaptic sOFF GCs. In this case, the tonic release of glycine from TRPV1 expressing ACs would likely directly inhibit which drives results in TRPV1 may In WT mice, OFF GCs receive cross-over inhibition from the ON pathway (Zaghloul et al., 2003; Margolis \& Detwiler, 2009; Roska \& Werblin, 2001; Roska et al., 2006; Chen et al., 2010) which decreases the spontaneous activity and enhances the peak and maintained rates to light decrements. Nobles and colleagues (2012) reported GlyRa2 mediated inhibition is involved in this cross- 
over pathway. In the absence of GlyRa2, spontaneous and evoked spike rates are decreased in sOFF GCs. This is similar to my results in the TrpV1\% sOFF GCs. Thus, TRPV1 activity may enhance glycinergic cross-over inhibition from the ON pathway to a subset of sOFF GCs sampled in vivo. These are presumably the sOFF alpha. This is consistent with my hypothesis that TRPV1 regulates tonic glycine release. TRPV1 positive ACs have processes in both the OFF and ON sublaminae, therefore, TRPV1 could reside in the narrow-field glycinergic AC providing the cross-over inhibition to the sOFF GCs.

Alternatively, TRPV1 could be acting in concatenated AC circuits. Such circuits have been observed (Anderson et al., 2011).

Another small population of OFF GCs showed TRPV1 modulation. TRPV1 activity shortened the response durations in approximately $25 \%$ of tOFF GCs which were sampled in vitro, but not in vivo. $\mathrm{GABA}_{\mathrm{c}}$ mediated inhibition to OFF BCs regulates the prolonged release of glutamate while glycine receptor mediated inhibition regulates both the peak and prolonged release of glutamate (Eggers \& Lukasiewicz, 2011). Since the peak rates in these GCs was similar to WT tOFF, this suggests TRPV1 mediated disinhibition of the GABAc feedback circuit, similar to what was proposed in the ON pathway. Alternatively, TRPV1 could be enhancing feed-forward inhibition to the subset of tOFF GCs. This could be from the TRPV1 positive ACs directly or again from concatenated AC circuits. Since these cells were not sampled in vivo, they are most likely not the transient OFF alpha cells and may have smaller axon diameters. TRPV1 reporter mice label populations of glycinergic ACs and OFF GCs which co-stratify in the center 
of the IPL (see TRPV1 expression chapter). The TRPV1 expressing GCs have a medium sized dendritic area and axon diameter and are a good target to investigate this possible circuit further.

\subsection{TRPV1 likely enhances sensitivity in the rod pathway via amacrine cell} mediated inhibition

TRPV1 enhances the sensitivity of responses to dim light. TRPV1 was necessary for signal processing through the primary rod pathway. Since ON and OFF responses were similar in vitro to 1.4 and $3.1 \log \mathrm{R}^{*} / \mathrm{rod} / \mathrm{sec}$ stimulation, secondary and tertiary rod pathways were intact in the TrpV1\%. The rod BC forms a synapse with the All AC. Rod derived excitatory information is transferred to the ON CBC through gap junctions. The All simultaneously inhibits the OFF CBC through a glycinergic synapse (Kolb \& Nelson, 1983; Strettoi et al., 1992; Chun et al., 1993; Merighi et al., 1996). Glycinergic inhibition of the OFF $\mathrm{CBC}$ during light stimulation enhances the response of the OFF $\mathrm{CBC}$ and $\mathrm{GC}$ to light offset (Merighi et al., 1996) Our results show that rod signaling through the rod to cone gap junctions and rod to OFF CBC is functional in TrpV1\%. Also, dark adapted b-waves which measures the depolarization of rod bipolar cells were similar to WT (Shen et al., 2009). Therefore, TRPV1 likely regulates signaling downstream from the depolarization of the RBC. This could occur due to inhibition of glutamate release from the RBC terminal, inhibition of the signal through the All AC, inhibition of glutamate release from the ON and OFF CBCs, or increased inhibition to ON and OFF GCs. My spiking responses alone cannot 
determine which process is responsible for the decreased rod sensitivity in ON and OFF GCs, however there are known pathways which share similarities with the probable pathways affecting the cone signals. One example is feedback inhibition to the RBCs. RBC to All is a dyad synapse between those two parties and the GABAergic A17 (Kolb \& Famiglietti, 1974; Famiglietti \& Kolb, 1975;

Strettoi et al., 1990; Strettoi et al., 1992; Chun et al., 1993; Kim et al., 1998). The A17 inhibits the RBC though feedback GABA release on the GABA receptors in the RBC terminal. Excessive $\mathrm{GABA}_{A}$ or $\mathrm{GABA}_{c}$ activation at the rod bipolar cell has been shown to decrease ON GC responsiveness to light in salamander (Ichinose and Lukasiewicz, 2002) and mice (Grimes et al., 2015). The A17mediated feedback loop improves the signal to noise ratio of rod signaling to light at the visual threshold. TRPV1 may be acting to modulate GABA release from the $\mathrm{A} 17 \mathrm{AC}$ and subsequent inhibition on the RBCs. More experiments are necessary to test this.

Disruption of signal through the All AC directly is through inhibition of the All or alterations within the coupling of the All network would also decrease signaling through the primary rod pathway. Light affects gap junctions in the retina. In the dark, the All AC network is weakly coupled and adaptation to a luminance values similar to twilight increases the coupling greater than ten-fold (see Bloomfield and Völgyi, 2009 for review) which enhances the signal through summation of synchronous activity. If TRPV1 regulated coupling, then in the absence of TRPV1, there may be an inability of the All network to regulate gap junctions. If coupling were increased in the dark, very small signals would spread 
to neighboring ACs and become dilute. Signals at twilight luminance levels, when networks are highly coupled, would be similar to WT. Under daylight conditions, coupling would decrease in the WT but not the TrpV1\%. Rod signaling should have minimal impact in the GC responses under these conditions. Direct inhibition of the All AC would have similar results. While this process is driven by light, it is mediated by release of dopamine from dopaminergic ACs (Hu et al., 2010; Kothmann et al., 2009; see Bloomfield and Völgyi, 2009 for a review). Dopamine acts through D1 receptors to modulate gap junction coupling and extend the dynamic range of light processing. This occurs in both the OPL and IPL and likely involves $\mathrm{GABA}_{c}$ receptors (Wellis \& Werblin, 1995; Herrmann et al., 2011; Smith et al., 2015). It should be noted that dopamine regulates gap junctions in all retinal layers. Furthermore, there is no evidence that coupling in specific layers is independent from the others. Alterations in dopamine signaling affects the b-wave amplitude and TrpV1- mice have normal b-waves (Jackson et al., 2014; Shen et al., 2009). For these reasons I find it unlikely that TRPV1 is regulating the dopaminergic system or gap junctions in the IPL. However, further studies are needed to investigate whether TRPV1 activity modulates this process.

\subsection{Possible methods of TRPV1 activation}

TRPV1 located in AC and GCs and likely regulates presynaptic release of neurotransmitter in the retina, consistent with the majority of evidence regarding the function of TRPV1 in the CNS (van der Stelt \& Di Marzo, 2004). TRPV1 activation often results in long after affects through influx and regulation of 
intracellular calcium concentrations at the terminals. Prolonged increased $\left[\mathrm{Ca}^{2+}\right]$ promote prolonged neurotransmitter release from the terminals (Medvedeva et al., 2008). and in several brain regions (Gibson et al., 2008; Maione et al., 2009; Kawahara et al., 2011 see Edwards, 2014 \& Ryskamp et al., 2014 for reviews) such as the hippocampus (Gibson et al., 2008). This process shapes pain receptor sensitization in the peripheral nervous system (Medvedeva et al., 2008) and long-term potentiation for memory formation in the hippocampus (Gibson et al., 2008). Our data suggest TRPV1 regulates presynaptic glycine release from the ACs it inhabits. TRPV1 most likely resides in the axon terminals of the GCs and is not mediating the visual responses in the retinal portion of the GCs directly (Chapter III). Figure 3.17 illustrates the potential involvement of TRPV1 in retinal serial inhibition. TRPV1 activation in amacrine cells regulates the tonic release of glycine onto glycine receptors located on another amacrine cell. That amacrine cell would subsequently be inhibited from releasing GABA or glycine onto their post-synaptic ON CBC or GC targets. In this way, TRPV1 enhances the spontaneous activity and maintained response by enhancing either tonic asynchronous glutamate release from ON CBCs or decreasing inhibition directly onto the ON GC. OFF GC responses are increased by enhanced cross-over inhibition during light stimulation, either from increased excitatory signaling in the ON pathway or the increased cross-over AC signal directly.

TRPV1 has numerous possible activators. The most likely activators in the retina are the endocannabinoids which are ubiquitously expressed in the retina (Yazulla, 2008). These include anandamide, NADA, and 
hydroperoxyeicosatetraenoic acids (HpETEs). Endocannabinoid release may be associated with bright light stimulation (Ryskamp et al, 2014). This is consistent with TRPV1 activity regulating luminance dependent enhancement of spontaneous activity and maintained rates in ON GCs. With regards to the role in visual processing, TRPV1 may be involved in regulating tonic release of neurotransmitters from ACs and in this way shapes the slower processes, the spontaneous and maintained responses, via tonic disinhibition of other ACs in a luminance dependent way. TRPV1 also enhances signal through the primary pathway in the dark. Further investigation is necessary to determine the mechanisms leading to this decreased sensitivity and the potential TRPV1 activator.

\section{Conclusions}

TRPV1 modulates visual signals in the retina. In the rod pathway, TRPV1 enhances sensitivity to dim light. In this way, TRPV1 increases the luminance range the retina can detect. In the cone pathway, TRPV1 prolongs the response duration of most sON GCs. In this way, TRPV1 likely enhances the perception of details in the visual world in daylight conditions. My data is consistent with a role for TRPV1 in modulating visual signals in the IPL, presynaptic to ON and OFF GCs. Specifically, TRPV1 enhances serial inhibition of ACs that release GABA onto $\mathrm{GABA}$ c receptors in ON BCs. This circuit optimizes the extraction of visual information that is relevant to dark or daylight conditions. The functional role of 
TRPV1 is consistent with its expression in amacrine cells. TRPV1 expression in the retina is discussed in the next chapter. 


\section{CHAPTER IV}

\section{TRPV1 IS EXPRESSED IN SPECIFIC AMACRINE AND GANGLION CELL CLASSES IN THE MOUSE RETINA}

\section{Introduction}

TRPV1 is a multi-modal receptor channel, which can activate or modulate neural signals both directly through influx and regulation of $\mathrm{Ca}^{2+}$ and indirectly through modulation of cellular pathways. TRPV1 has been extensively studied for its role in nociception, inflammation, and pathology in the PNS and CNS. A role for TRPV1 in retinal pathology has only recently been proposed and is less well understood. TRPV1 activity has been implicated as both neuroprotective and neurotoxic in diabetic retinopathy (Krassas et al., 2007), ocular ischemia (Sakamoto et al., 2014) and glaucoma (Sappington et al., 2015 for review). For example, TRPV1 mediated processes have recently been implicated as a contributing factor in GC death in mouse models of glaucoma in vitro (Sappington et al., 2009), but TRPV1 activation may be protective to GCs in the DBA/2J in vivo mouse models of glaucoma (Ward et al., 2014; see Sappington et al., 2015 for review). 
Ritter \& Dinh (1988, 1990, and 1992) observed the TRPV1 agonist capsaicin induced death of cells in the INL and GCL and in axon terminals in brain nuclei, which were targets of GCs. Specifically, Ritter and Dinh reported neurodegeneration in the suprachiasmatic nucleus $(\mathrm{SCN})$, ventrolateral geniculate nucleus (vLGN), intergeniculate leaflet (IGL), and olivary and medial pretectal nuclei (OPN \& MPN). Systemic capsaicin induced neurodegeneration in visual nuclei was eliminated when the eye was enucleated prior to capsaicin injection (Ritter \& Dinh, 1992). Some GC axons were damaged by capsaicin while somas remained intact. This suggests capsaicin neurotoxicity is mediated by TRPV1 in mouse GC axons and inner nuclear retinal neurons. However, while capsaicin induces neurodegeneration through TRPV1 mediated excitotoxicity, it also induces damage through TRPV1-independent mechanisms (Kim et al., 2003; Costa et al., 2005; Athanasiou et al., 2007; see Ryskamp et al., 2014 for a review). Also, capsaicin is a known agonist of TRPM1 channels, the signaling channel in ON BCs in the retina. TRPM1 mediates capsaicin evoked currents in ON BCs of TrpV1\% mice but not TrpM1\% (Shen et al., 2011; Ray et al., 2014). Therefore, further studies are needed to determine TRPV1 expression in retina. I tried to use various commercially available TRPV1 antibodies and found that different antibodies produced different expression patterns and the results were inconclusive. As an alternative, I crossed TRPV1 ${ }^{\text {cre }}$ to Ai9 reporter mice and assessed TRPV1 expression throughout development. In comparison, I used intravitreal viral delivery of the LoxP-tdTomato construct to TRPV1 ${ }^{\text {cre }}$ retinas to assess TRPV1 expression in the mature retina. I determined that: 
1. TRPV1 is localized to two narrow-field (NF) AC classes, one wide-field (WF) displaced AC class, and three GC classes in the mature mouse retina.

2. Axons from TRPV1 expressing GCs target the SCN, vLGN, IGL, and OPN, the previously reported sites of capsaicin induced neurodegeneration (Ritter \& Dinh, 1992) as well as the superior colliculus (SC).

3. TRPV1 gates excitatory current in ACs but not in GCs, suggesting TRPV1 plays a role in presynaptic modulation of visual signal.

Clarifying the specific expression of TRPV1 in the retina and functional role in normal visual signaling or pathology is important if either agonists or antagonists of TRPV1 are to be pursued as possible therapeutic options. Here I investigated the localization of TRPV1 in the mouse retina.

\section{Results}

\subsection{TRPV1 antibodies are unreliable in the mouse}

TRPV1 is expressed in the rodent CNS at levels $\sim 20-30$ fold lower than in the dorsal root ganglia (Sanchez et al., 2001; Cavanaugh et al., 2011b). The presence of TRPV1 mRNA and protein in the mouse (Shen et al., 2009; Gilliam et al., 2011; Sappington et al., 2011), rat (Nucci et al., 2007) and human (Martinez-Garcia et al., 2013) retina has been confirmed. The exact localization of TRPV1 in retina is under debate (see Ryskamp et al., 2014 for a review) but the majority of studies support TRPV1 expression in the inner retina and microglia in rodent retina. Sappington and colleagues report expression of 


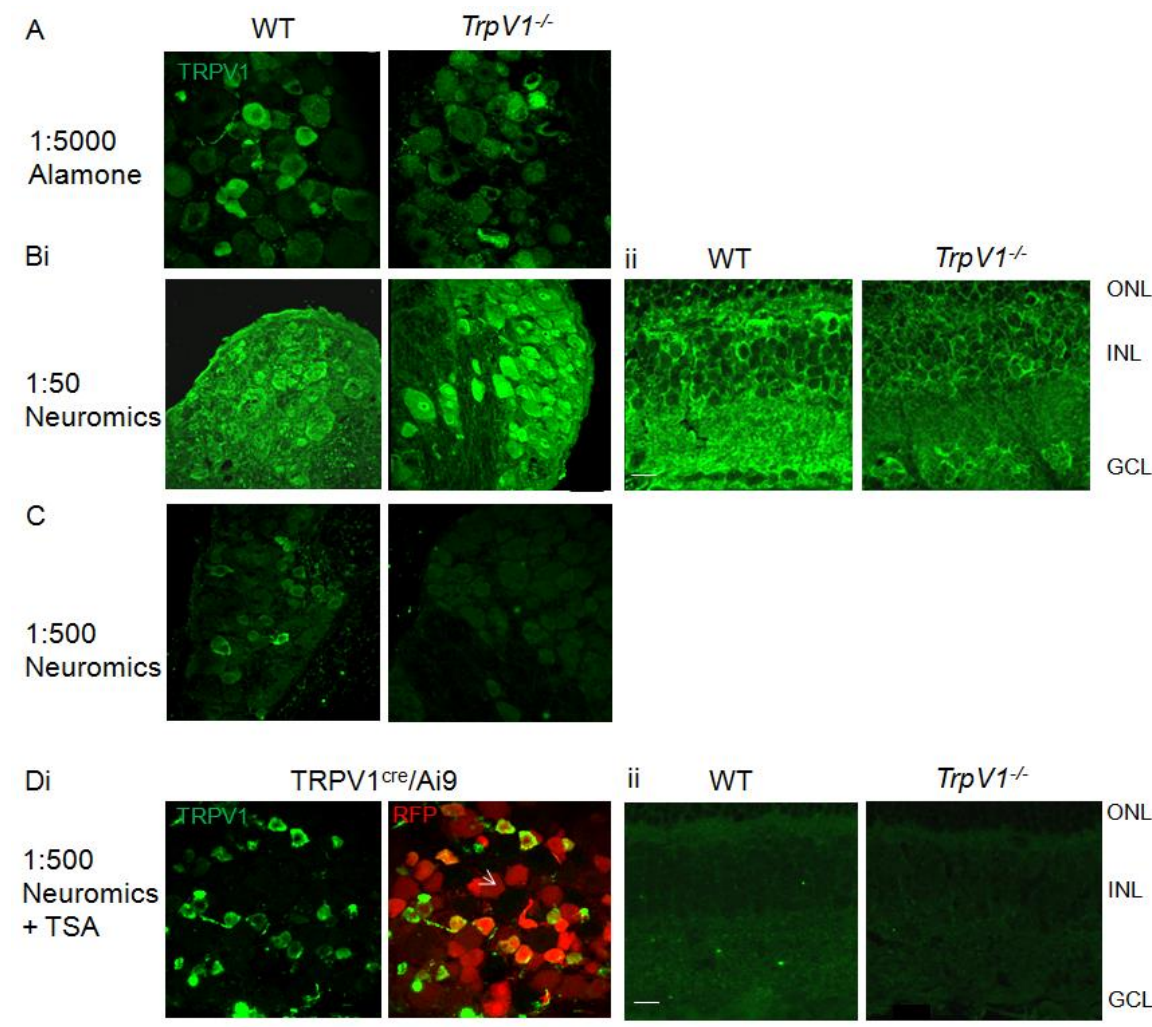

Figure 4.1. TRPV1 antibody label had a low signal to noise ratio in mouse DRG and retina. (A) Rabbit anti-TRPV1 from Alamone Labs labels a non-specific protein in WT and TrpV1\% DRG. Scale bar $20 \mathrm{um}$. (B) High concentration rabbit anti-TRPV1 from Neuromics (1:50) produces non-specific label in DRG (Bi) and retina (Bii; scale bar 10 um), but low TRPV1 specific label at more dilute concentrations (C; 1:500). (Di) The amplified Neuromics antibody label in TRPV1 1 re/Ai9 reporter DRG is co-localized with tdTomato positive neurons. A population of primary afferents expressed TRPV1 transiently during development and therefore was tdTomato positive but negative for TRPV1-ir (arrow). (Dii) Amplified TRPV1-ir is low and punctuate in WT retina and absent in $\operatorname{TrpV} 1 \%$ retina. Scale bar $10 \mathrm{um}$.

TRPV1 in GCs of mice using in situ hybridization (2015). TRPV1 antibody expression has been variable and inconsistent across several reports (see Ryskamp et al., 2014 for a review), confirming the need for proper controls to interpret the data. $\operatorname{TrpV} 1 \%$ mice were not used in any of the previous studies to confirm specific expression of the antibody.

To determine the specific expression of TRPV1 in the retina, I tested all of the antibodies listed in prior publications (Table 2.1). Further I used them at the 
published concentrations and also performed a concentration gradient to determine the optimal concentration for each antibody. I compared retinal expression to the firmly characterized dorsal root ganglia (DRG) expression in TRPV1 reporter mice. To confirm the specificity of TRPV1 antibody immunoreactivity (ir), I simultaneously labeled WT and TrpV1\% retina and DRG. In my experience, TRPV1 antibodies were unreliable in the mouse for the following reasons: TRPV1-ir from several antibodies had a low signal to noise ratio in the DRG, which I enhanced using tyramide signal amplification (TSA). One antibody (Alamone Labs; Table 2.1) did not specifically target TRPV1; TRPV1-ir was still present in the DRG of TrpV1/- mice (Fig. 4.1a). Rabbit antiTRPV1 from Neuromics used at previously reported concentrations (Neuromics, 1:50; Sappington et al., 2009, 2015) also resulted in non-specific label in the both WT and $\operatorname{TrpV1}$ - DRG and retina (Fig. 4.1bi \& bii). When I decreased the concentration (1:500) I obtained label in the WT DRG, which was absent in the $\operatorname{TrpV} 1 \%$. That indicated the possibility that the antibody had specificity at that concentration (Fig. 4.1ci). When the same concentration was used in retinal sections, I found faint punctate signal in the IPL that was only slightly brighter than the $\operatorname{Trp} V 1 \%$ retina. As a consequence, I tried Tyramide Signal Amplification (TSA) to enhance the label. I tested the system first in the DRG of TRPV1 ${ }^{\text {cre }} /$ Aig reporter mice. In DRG slices with TSA from four mice, $27 \%$ of neurons with TRPV1-ir were still faintly labeled (26/96). All neurons with TRPV1-ir coexpressed tdTomato (Fig. 4.1di). Some (60\%) DRG neurons expressed tdTomato but no TRPV1-ir (arrow) indicative of transient TRPV1 expression in a 
population of primary afferent during development, as has been reported (Mishra et al., 2011). Even with TSA, TRPV1-ir was low in retina and possibly restricted to small puncta in the IPL. The low signal to noise ratio of TRPV1-ir in retina lead me to conclude the antibodies were not useful for determining TRPV1 expression there. I attempted to enhance the signal of that and the other antibodies using various techniques which had been useful with other antibodies (data not shown). I fixed the tissue with various concentrations of paraformaldehyde and for various lengths of time. I also tried antigen retrieval. I was unable to enhance the signal to noise ratio any further than I did with the TSA. As an alternative to the antibodies, I used TRPV1 reporter mice to investigate the specific expression of TRPV1 in the mouse retina.

\subsection{TdTomato expression in TRPV1cre/Ai9 and TRPV1cre $-A A V$ retinas was similar in the GCL and differed in the INL}

Figure $4.2 \mathrm{a}$ was a $500 \mu \mathrm{m} \times 500 \mu \mathrm{m} 3 \mathrm{~d}$ reconstruction of the retina from the INL to the GCL obtained from a TRPV1 1 cre /Ai9 mouse. Vertical sections showed the processes of labeled cells overlapped fairly continuously in the IPL throughout the retina (Fig. 4.2c) and stratified densely in sublaminae one, two and three above the bottom CHAT band. There was sparse labeling in s4 and s5. Images were collected from the dorsal, ventral, temporal and nasal quadrant of four retinas. The mean number of labeled cells in four $0.25 \mathrm{~mm}^{2}$ areas of the GCL and INL from three mice was $26( \pm 3.6)$ and $220( \pm 30.5)$ respectively (Fig. 4.2b). The density of tdTomato+ cells was $104 / \mathrm{mm}^{2}$ in the $\mathrm{GCL}$ and $880 / \mathrm{mm}^{2}$ in 
the INL. TdTomato was expressed in all areas of the retina. There were similar

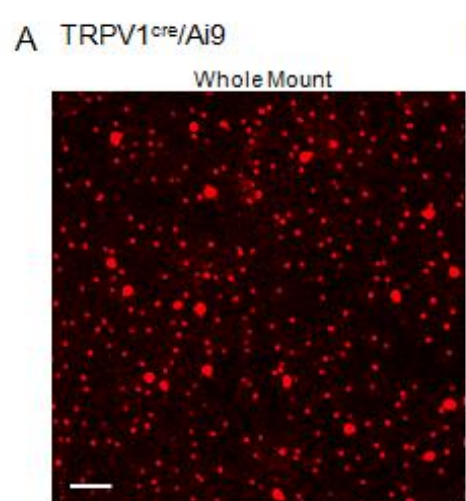

B
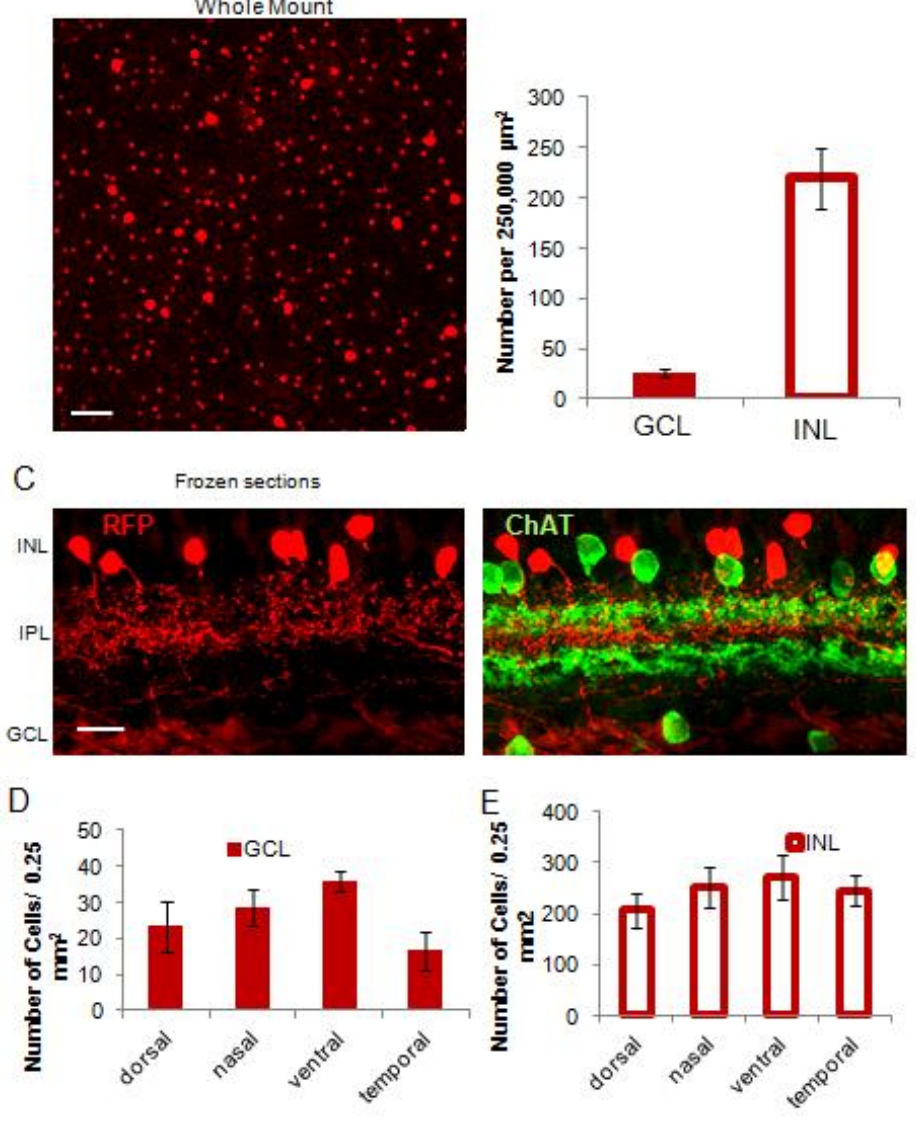

Figure 4.2. TdTomato is expressed in the INL, IPL \& GCL of TRPV1 ${ }^{\text {cre }} / A$ i9 retinas. (A) Cells in the INL and GCL are labeled consistently across the retina. Scale bar $50 \mu \mathrm{m}$ (B) The GCL has an average of $26( \pm 3.6)$ labeled cells and the INL has $220( \pm 30.5)$ in an area similar to that shown in (A). (C) Processes of labeled cells form a plexus in s1-3 in the IPL across the retina and majority of processes stratify betlen the ChAT bands. Sparse processes also populate $s 5$. Scale bar $15 \mu \mathrm{m}$. (D) The distribution of cells in the GCL and INL is mostly consistent across the retina except there are felr labeled in the temporal GCL. numbers of tdTomato

positive (tdTomato+) cells

in the GCL (Fig. 4.2d;

ANOVA $p=0.16$ ) and INL

(Fig. 4.2e; ANOVA

$p=0.67$ ) between all

quadrants. Assuming a

mouse retinal area of 14

$\mathrm{mm}^{2}$, I calculate that there

were 1456 cells in the

GCL and 12,320 cells in

the INL that expressed

TRPV1 (Williams \&

Goldowitz, 1992;

Lyubarsky \& Pugh, 1996).

Approximately $3 \%$ of $\mathrm{ACs}$

in the INL are cholinergic

starburst ACs, which form

the OFF ChAT band in the

IPL. These ACs have a density of $1100 / \mathrm{mm}^{2}$ (Jeon et al., 1998). TRPV1cre/Ai9

tdTomato+ cells in the INL were presumably ACs based on small soma size and absence of processes that projected to the OPL and comprised $\sim 2.4 \%$ of all 
ACs. Similarly, W3 GCs are the most numerous in mouse retina, comprising $\sim 13 \%$ of all GCs with a density of $250 / \mathrm{mm}^{2}$ (Zhang et al., 2012). The density of tdTomato+ GCL cells was less than half of that.
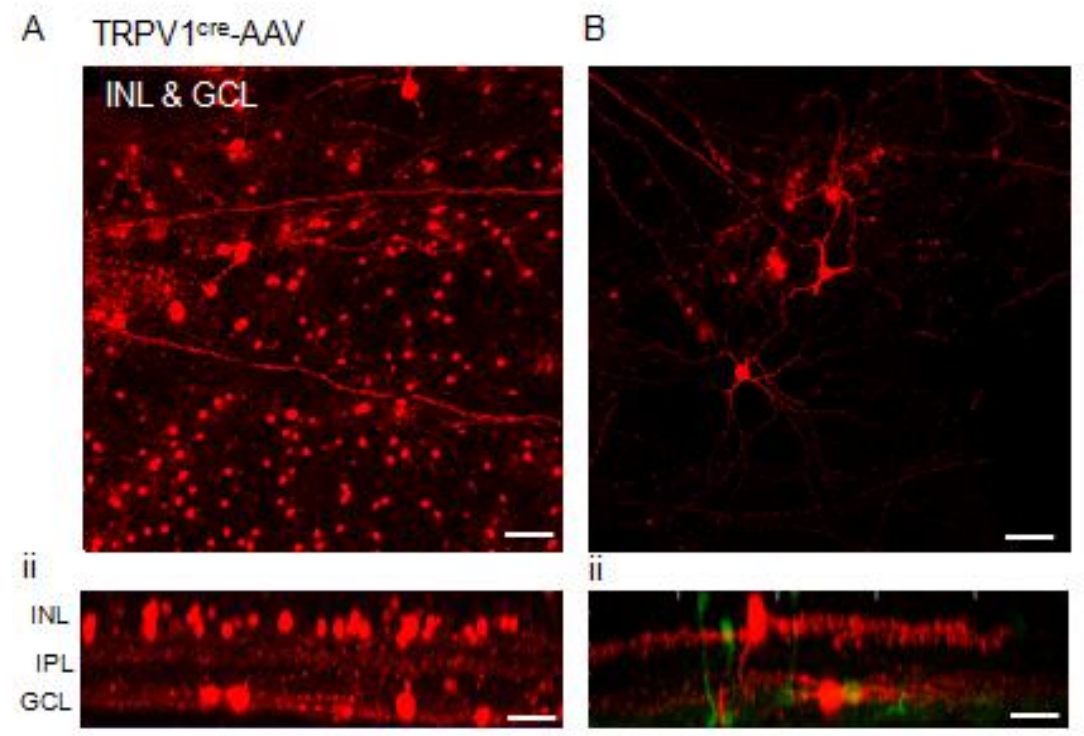

Figure 4.3. TdTomato expression is similar in TRPV1 ${ }^{\text {cre }} / A i 9$ and TRPV1 ${ }^{\text {cre }}$-AAV retinas. (A) Cells in the INL and GCL are labeled consistently across areas of retina with dense expression. Scale bar $50 \mu \mathrm{m}$. An enlarged side projection of $A$ shows a similar dense plexus in s1-3 (B; scale bar $20 \mu \mathrm{m}$ ). (C) The injection techniques resulted in areas of sparse label. Individual cells were isolated enough to morphologically characterize. Axons confirm the 3 large cells in this picture are GCs. Several ACs with small somas are also labeled. (D) One of the GCs stratifies in the ON sublaminae, while another has a displaced soma in the INL, thick processes in s1, and an axon projecting through the IPL to the nerve fiver layer (arrow). Scale bar $20 \mu \mathrm{m}$.

TRPV1 ${ }^{\text {cre }}$ /Ai9 tdTomato expression does not differentiate transient developmental and mature TRPV1 expression. Also, the overlap of tdTomato+ processes precludes morphological identification of many of the individual cell classes. To address both issues, I intravitreally injected mature TRPV1 ${ }^{\text {cre }}$ mice with AAV containing the LoxP-tdTomato construct. Retinas had patches of either dense (Fig. 4.3a \& b) or sparse (Fig. 4.3c \& d) tdTomato label. The decreased tdTomato expression in sparse areas was indicative of decreased viral 
integration in those cells. Because of the variability in expression, I compared the patterns of tdTomato expression in densely labeled areas to TRPV1 ${ }^{\text {cre }} / \mathrm{Ai9}$, but not the numbers of labeled cells. I also isolated individual cell types in sparsely labeled areas and compared them to cell types found in TRPV1 ${ }^{\text {cre }} /$ Ai9. Those results were described in later sections.

The densely labeled areas had similar, but not identical, expression patterns to the TRPV1 ${ }^{\text {cre }} /$ Ai9 (Fig. 4.3a). Cells were labeled in the GCL and INL. Many in the GCL were had axons and were GCs. Cells in the INL had processes projecting to the IPL only, characteristic of ACs. The enlarged transverse view shows the tdTomato processes overlapped extensively in s 1-3. In the ON sublaminae of the TRPV1 $1^{\text {cre}} / \mathrm{Ai9}$, there were isolated NF processes that were discernibly stratified in s 4-5. There were also WF processes in s 5 . In contrast,

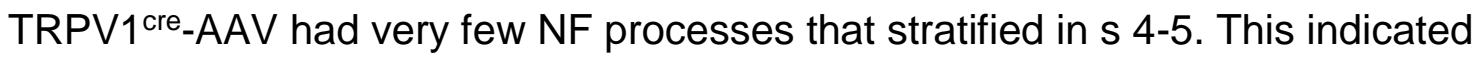
the latter tdTomato+ ACs expressed TRPV1 transiently in development.

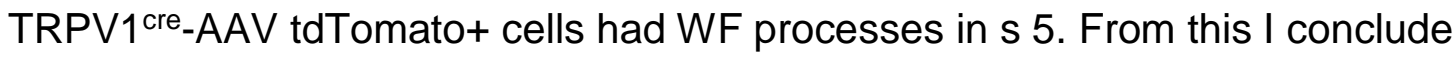
TRPV1 is expressed in OFF stratified ACs in GCs in the mature retina. ON stratified NF ACs had transient TRPV1 expression during development. I next assessed the morphology of TRPV1 expressing cells.

\subsection{TRPV1 was expressed in two NF and one wide-field amacrine cell class in the mature retina}

Individual ACs were isolated in the sparsely labeled areas of wholemount

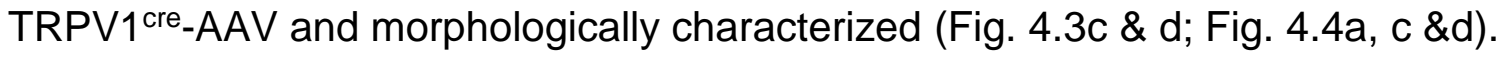

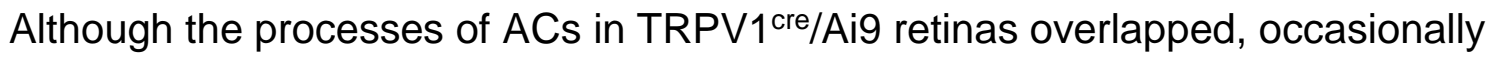


the dendritic tree that projected from an individual NF AC process was discernible in transverse slices (Fig. 4.4b arrow). The dendrites of those ACs were not isolated and the origin of adjacent processes could not be determined (arrowhead). Dendritic and soma areas and stratification were only measured on isolated TRPV1 ${ }^{\text {cre }}$-AAV ACs (Fig. 4.4a, c \& d). ACs observed in TRPV1 ${ }^{\text {cre/Ai9 }}$ were only used for qualitative comparison to similar morphological types which were isolated in the TRPV1-AAV (Fig. 4.4a). Two classes of NF ACs, which comprised $86 \%$ isolated ACs, were identified in the retinas of both TRPV1 ${ }^{\text {cre }}$-AAV and TRPV1 ${ }^{\text {cre }} /$ Ai9. The majority of isolated NF ACs $(n=17,61 \%)$ resembled the Flag A ACs identified by MacNeil and colleagues (1999) in the rabbit retina (Fig. 4.4a). Flag A ACs stratified in s2-3 of the IPL.

One NF AC that resembled the Flag AC that stratified in $\mathrm{s} 4-5$ was isolated

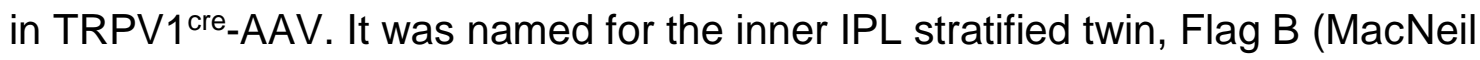
et al., 1999). The second NF AC class that was regularly isolated in TRPV1 ${ }^{\text {cre }}$ AAV retinas $(n=7,25 \%)$ resembled the "no glycine, no GABA" (nGnG) ACs described in the mouse retina (Fig. 4.4a; Kay et al., 2011). nGnG ACs received that name due to the absence of the inhibitory neurotransmitters, which are typically found in ACs. nGnG ACs had similar sized dendritic areas as the Flag A, but processes stratified in s1-3. NF ACs that had similar stratification patterns were observed in TRPV1 ${ }^{\text {cre }} /$ Ai9 transverse sections (Fig. 4.4b). The NF AC that had ON stratified processes and was observed in the TRPV1 ${ }^{\text {cre }} /$ Ai9 but not TRPV1 ${ }^{\text {cre }}-A A V$ appeared to be multistratified in all IPL sublaminae. That AC resembled the rabbit $A B$ diffuse 1 (Fig. 4.4b; MacNeil et al., 1999), which also 


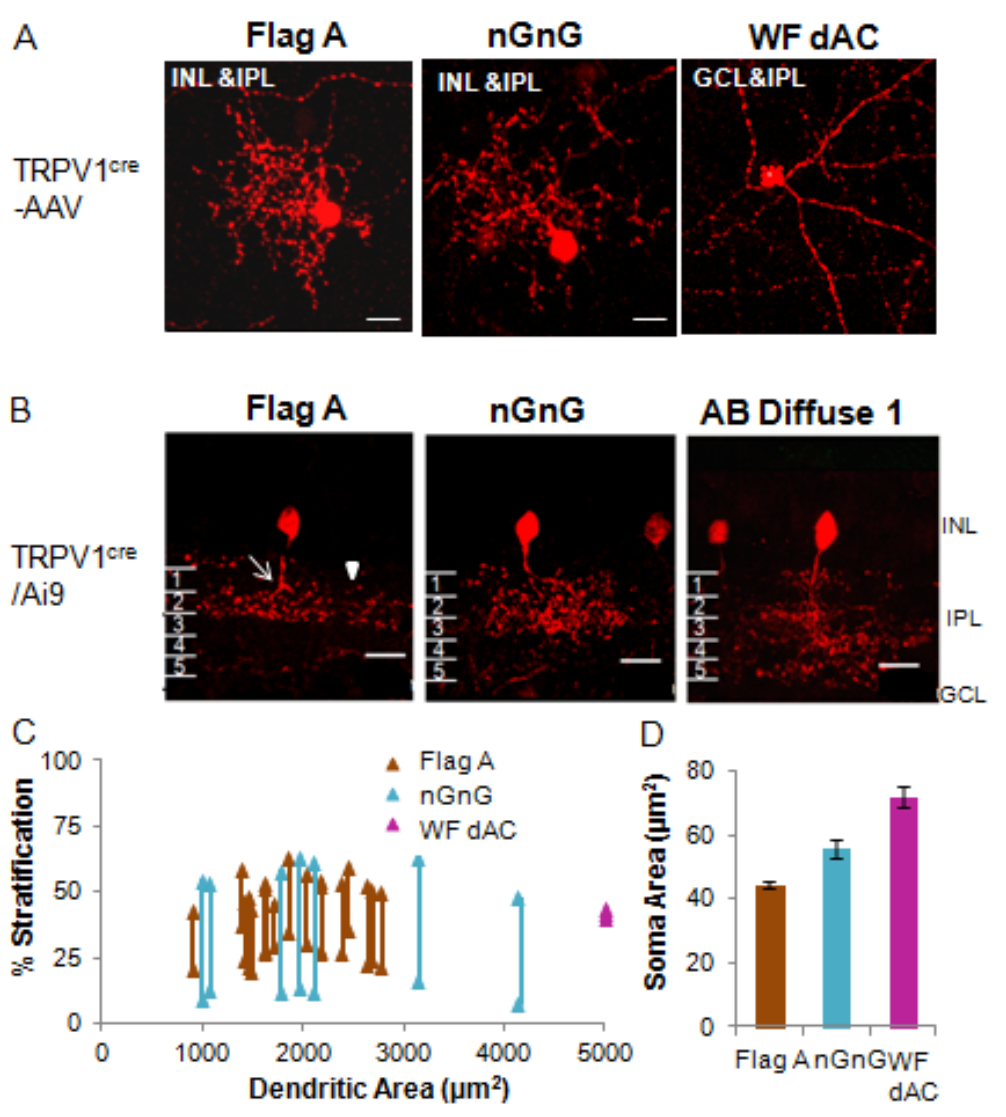

Figure 4.4. TRPV1 is expressed in mouse ACs (Ai) Flag A ACs were observed in the TRPV $1^{\text {cre }} / \mathrm{Ai} 9$ and TRPV $1^{\text {cre }}$-AAV (Bi; scale bar $10 \mu \mathrm{m})$. The narrow-field dendrites $\left(1960 \pm 378 \mu \mathrm{m}^{2}\right)$ stratified in s2-3 (Bi \& C). ( $\mathrm{Ai})$ nGnG ACs also were observed in both reporters (Bii; scale bar $10 \mu \mathrm{m}$ ) and had slightly larger dendritic areas $\left(2270 \pm 530 \mu m^{2}\right)$. nGnG ACs stratified in s1-3 (Bii\&C). (Aiii) The wide-field WA3-2 dAC was observed in sparsely labeled areas of TRPV1 1 cre-AAV and stratified in the middle of the IPL at the border of the ON and OFF sublaminae (C). (Biii) AB-diffuse 1 ACs were multistratified throughout the IPL and were only observed in TRPV 1 cre/Ai9, suggesting TRPV1 is transiently expressed during development. Scale bar $10 \mu \mathrm{m}$. (D) Soma areas increase in relation with dendritic areas. stratified in all

sublaminae. $A B$

diffuse 1 ACs were

discernible in

wholemount

TRPV $1{ }^{\text {cre }}-\mathrm{Al9}$ due to

the isolated NF

processes in the $\mathrm{ON}$

sublaminae. I

assigned the

corresponding

processes in the OFF

sublaminae to the

same $A C$, but it was

possible the latter

processes originated

from neighboring $A C s$.

If that were the case,

NF ON processes

were from Flag B type

ACs instead. TdTomato+ ON stratified NF ACs expressed TRPV1 during

development, but not in the mature retina. In contrast, TRPV1 was expressed in

Flag $A$ and $n G n G$ NF ACs in the mature retina. 
TdTomato also was expressed in a WF AC with a soma displaced to the GCL (dAC) (Fig. 4.4a; $n=3,11 \%$ ). Those dACs lacked axons in the nerve fiber layer, a defining characteristic that delineated dACs from GCs. TdTomato+ WF dACs resembled the WA3-2 (Pérez De Sevilla Müller et al., 2007) and the bifid (Badea \& Nathans, 2004). The WF ACs described in both publications had two groups of sparsely branching processes projecting radially from conventionally placed somas in the INL. The processes stratified in s3 at the border of the ON and OFF sublaminae. Because the somas of tdTomato+ WF dACs in the TRPV1 ${ }^{\text {cre }}$-AAV were displaced to the GCL, I did not assign them the classification names above. A WF dAC with similar morphology was recently described in neuronal nitric oxide synthase (nNOS) reporter mice (Zhu et al., 2014). The expression of nNOS should be confirmed before assigning WF dACs in TRPV1 1 cre/AI9 retinas the nNOS classification and will be the subject of future experiments. WF dACs were not isolated enough to identify in TRPV1 ${ }^{\text {cre/AI9 }}$ retinas, but I did find somas in the GCL that lacked axons (Fig. 4.5d). Glycine is expressed in NF ACs, while medium and WF are GABAergic (Marc et al., 1995). As mentioned, nGnG NF ACs are the exception to that rule. I labeled the TRPV1 expressing (TRPV1+) ACs nGnG based on a similar morphology to those published. The neurotransmitter associated with those cells was still unknown. I next looked for the presence of inhibitory neurotransmitters by labeling retinal slices and wholemounts with antibodies to glycine, GABA or the GABA synthesizing enzyme, Glutamic acid decarboxylase isoforms $65 \& 67$ (GAD65/67). The wholemount labeling showed the majority of tdTomato+ ACs 


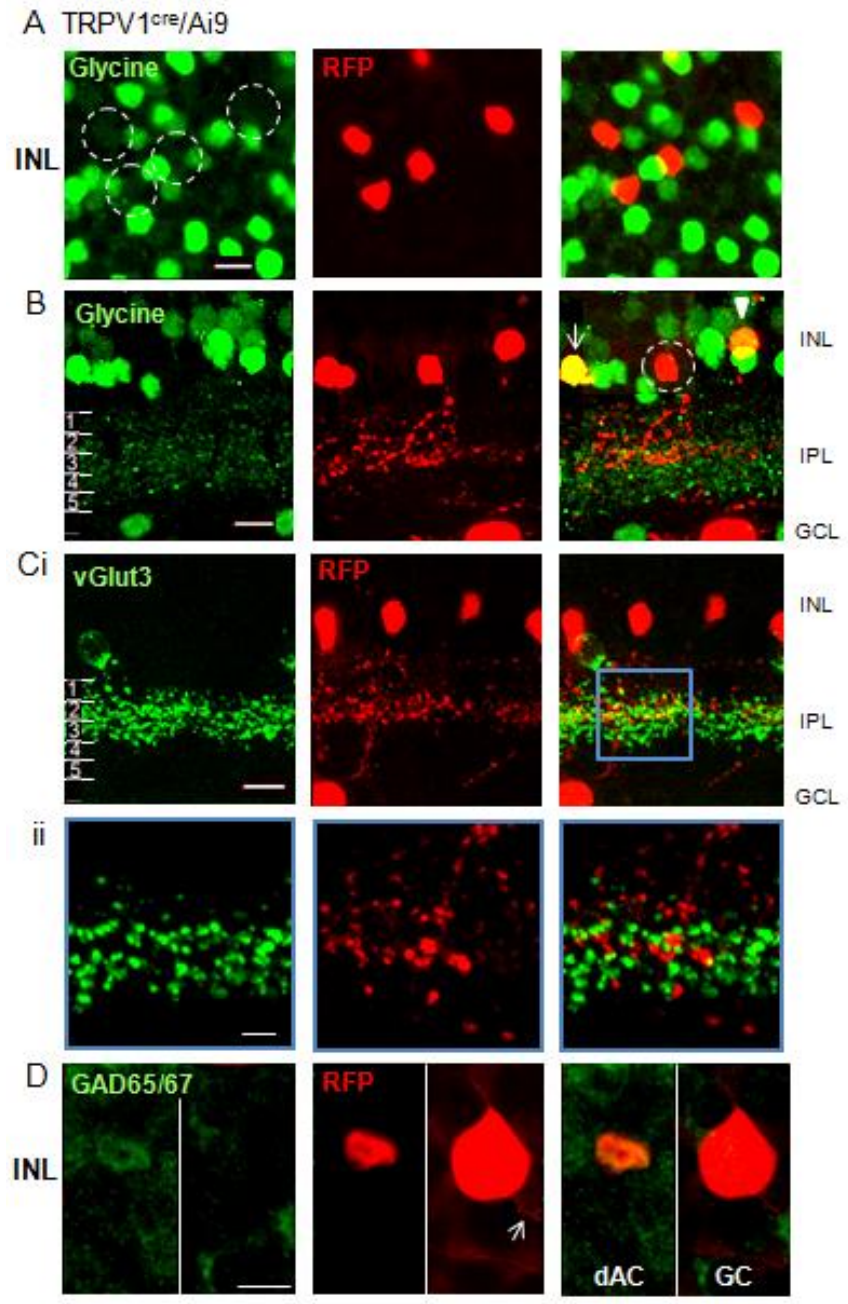

Figure 4.5. Neurotransmitter expression in TRPV1+ ACs. (A) Wholemount TRPV1 1 cre/Ai9 retinas were colabeled for glycine and RFP. A subset of TRPV1+ ACs had negligible (broken circles) or no (solid circle) glycine expression. The morphology resembles the nGnG (B; Kay et al., 2014). Neighboring ACs have high (arrow) or moderate (arrowhead) glycine expression. (C) TRPV1+ ACs do not express vGlut3, holver an enlarged view of the IPL show processes co-stratified with and directly adjacent to glutamatergic ACs. Scale bar is $10 \mu \mathrm{m}$ in A, B \& Ci. Scale bar is $4 \mu \mathrm{m}$ in Cii. were glycinergic, but there

was a population of ACs,

which had very low

(broken circles) or

undetectable (solid circle

levels of glycine (Fig.

4.5a). The somas of

nGnG ACs did not

express GABA either. The

transverse view showed

the processes of these

ACs stratified in s 1-3,

similar to the nGnG ACs

reported by Kay and

colleagues (2011). Figure

$4.5 b$ showed an isolated

nGnG AC (circle) in slice

with processes largely

ramified in s 2-3. Other

tdTomato+ ACs

expressed both high

(arrow) and low (arrowhead) levels of glycine. 
Flag A ACs were first characterized in rabbit retina. The most similar morphological correlate that had been described in mouse were the glutamatergic ACs (GACs), which expressed glycine and vesicular glutamate transporter type 3 (vGlut3; Haverkamp \& Wässle 2004; Lee et al., 2014; Kim et al., 2015). I labeled TRPV1 ${ }^{\text {cre }} /$ Ai9 retinal slices with an antibody to vGlut3 to determine if TRPV1 was expressed in that subset. While tdTomato+ ACs costratified with GACs, they were not labeled (Fig. 4.5c). Further, the processes of the two types of ACs were adjacent to each other which could suggest a possible interaction, though more information is necessary to confirm that idea (Fig. 4.5cii; see discussion). GAD65/67 antibody labeled few, sparsely distributed tdTomato+ dACs, which were presumed to be the WF dACs isolated in the TRPV1 ${ }^{\text {cre }}$-AAV retinas. The dACs in TRPV1 ${ }^{\text {cre }} / \mathrm{Ai9}$ have a very low density of approximately 4/mm². I concluded that TRPV1 largely resided in NF ACs, which stratified in s 13. The majority of these were glycinergic and among the others I have not been able to identify their associated neurotransmitter.

\subsection{TRPV1 was Expressed in One OFF and Two Intrinsically Photosensitive Retinal Ganglion Cell Classes}

To determine the morphologies of TRPV1 expressing GCs in the mature and developing mouse retinas, isolated GCs were imaged in the TRPV $1^{\text {cre }}-\mathrm{AAV}$ mice and compared to GCs in the TRPV1 ${ }^{\text {cre}} /$ Ai9. TdTomato+ GCs in the TRPV1 ${ }^{\text {cre/Ai9 }}$ were filled with Lucifer Yellow and/or Neurobiotin for morphological characterization (see methods). Three GC classes were labeled in both

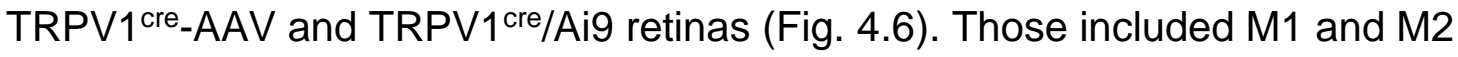



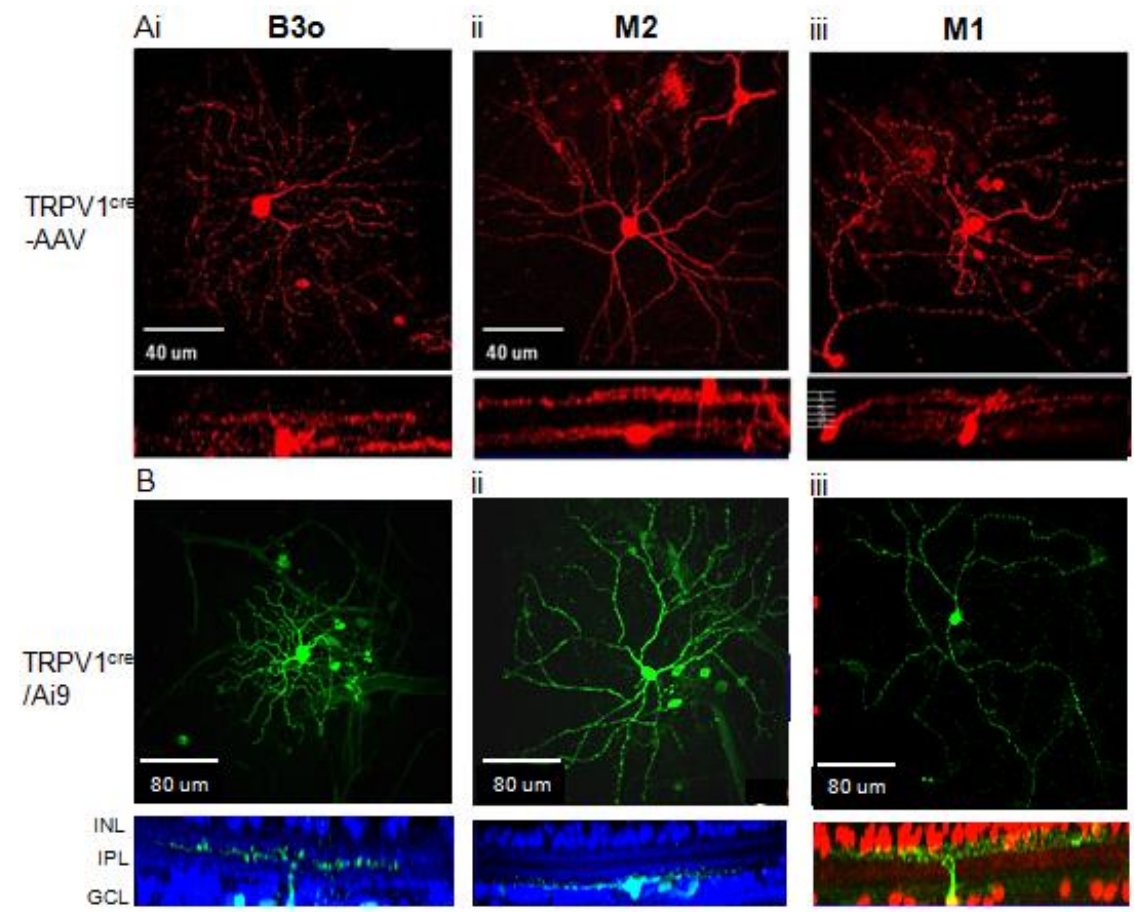

C
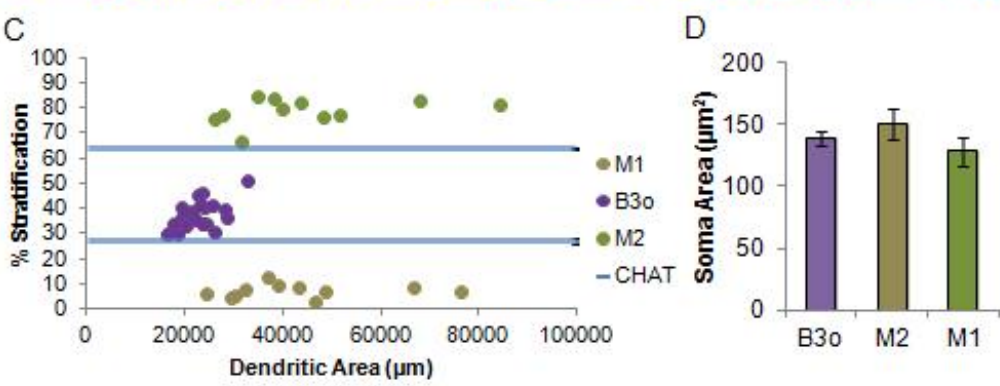

Figure 4.6. TRPV1 is expressed in mouse GCs (Ai) B3o GCs were observed in the TRPV1 1 re/Ai9 and TRPV1 1 re-AAV (Bi). The medium-field dendrites $\left(22,065 \pm 915 \mu \mathrm{m}^{2}\right)$ stratified in s3 between the middle of the IPL and the OFF ChAT band (Ai, Bi \& C). (Aii) TdTomato+ M2 GCs were observed in both reporter mouse types (Bii). M2 dendrites stratified in s5 of the IPL (mean area: 44,677 $\pm 5,100 \mu \mathrm{m}^{2}$; Bii \& C). (Aiii) M1 GCs had few deep diving processes, which stratified in $\mathrm{s} 1$ and spread over a large area $\left(43,550 \pm 5,640 \mu \mathrm{m}^{2}\right.$; Biii \& C). Scale bar is $20 \mu \mathrm{m}$ in A and $40 \mu \mathrm{m}$ in B. (D) Soma area were similar between classes.

intrinsically photosensitive (ip) GCs (Berson et al., 2010) and one OFF GC. The morphologies of M1 and M2 GCs have been extensively characterized (see Schmidt et al., 2011 for a review) and TdTomato+ M1 and M2 GCs had similar morphologies. M1 GCs had few dendrites, which stratified in s1 of the IPL (Fig. 
4.6a, b \& c; Berson et al., 2010; Ecker et al., 2010). M2 GC had more dendrites and regular pattern in the ON sublaminae (Fig. 4.6 a, b \& c; Berson et al., 2010). The tdTomato+ M1 and M2 GCs had variable dendritic and soma areas, their distributions overlap, and the M1 mean dendritic area was slightly smaller than M2 (M1: $n=11$ dendritic area: $43,550 \pm 5,640$, soma area: $128.4+11.1 ; M 2: n=12$ dendritic area: $44,677 \pm 5,100$, soma area: $150.8+12$ ). All of my measurements were similar to those described by Berson and colleagues (2010). The OFF GC had monostratified processes in $\mathrm{s} 3$ at the border of ON and OFF IPL layers $(n=23 ; 46 \pm 1 \%)$. The medium sized dendritic area $\left(22,065 \pm 915 \mu m^{2} ;\right.$ Fig. $4.6 b$ \& c), was similar to B3o (199 $\mu \mathrm{m}$ dendritic diameter, $15 \mu \mathrm{m}$ soma diameter; Sun et al., 2002). The B3o had the smallest dendritic area of the tdTomato+ GCs (Fig. 4.6c) but a similar soma size to the tdTomato+ positive M1 and M2 GCs (Fig. 4.6d). All three GC classes labeled in the TRPV1 ${ }^{\text {cre/Ai9 }}$ also were represented in the TRPV1 ${ }^{\text {cre }}-A A V$ retinas. M1 GCs were most frequently labeled $(n=17,55 \%)$, followed by M2 $(n=6,19 \%)$ and B3o $(n=6,19.4 \%)$. A small number of other GCs were observed. A multi-stratified GC with small dendritic area $\left(19,423 \mathrm{um}^{2}\right)$ was labeled in a TRPV1 ${ }^{\text {cre }} / A i 9(n=1)$ and TRPV1 ${ }^{\text {cre }}-\operatorname{AAV}(n=1)$ retina. The dendrites stratified in s 1-3 and resembled the W3 (Zhang et al., 2012). One large ON GC which resembled the A1 (Sun et al., 2002) and M4 (Estevez et al., 2012) was identified in a TRPV1 ${ }^{\text {cre }}$-AAV retina. Because morphologically similar GCs

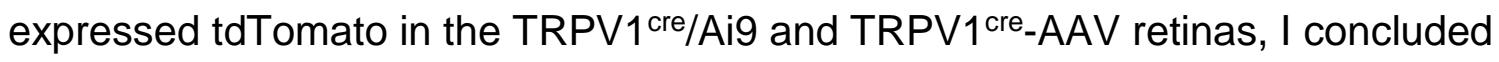
that TRPV1 is expressed in M1, M2 and B3o GCs in the mature retina.

TdTomato+ GCs were referred to as TRPV1+ for all subsequent analysis. 
M1 and M2 ipGCs express the photopigment melanopsin (Berson et al., 2010). I next confirmed the expression of melanopsin in TRPV1+M1 and M2 GCs. I labeled three TRPV1 ${ }^{\text {cre }} / \mathrm{Ai9}$ retinas with an antibody to melanopsin using tyramide signal amplification. The melanopsin antibody label in TRPV1 ${ }^{\text {cre/Ai9 }}$ retinas matched previous publications (Estevez et al., 2012). M1 GCs have bright

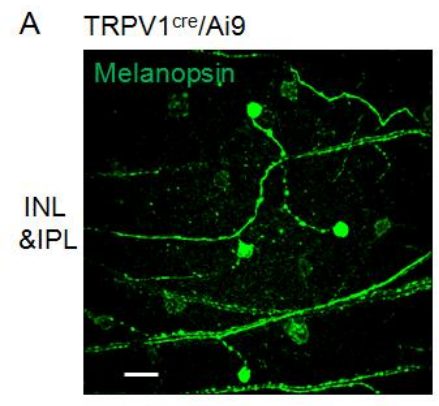

B

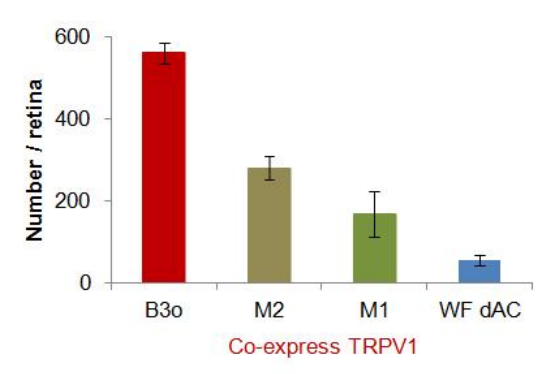

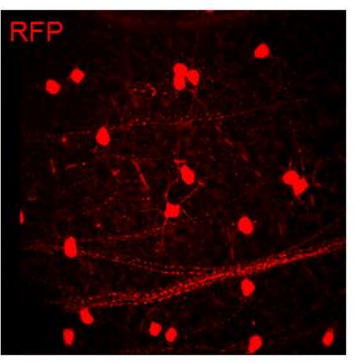
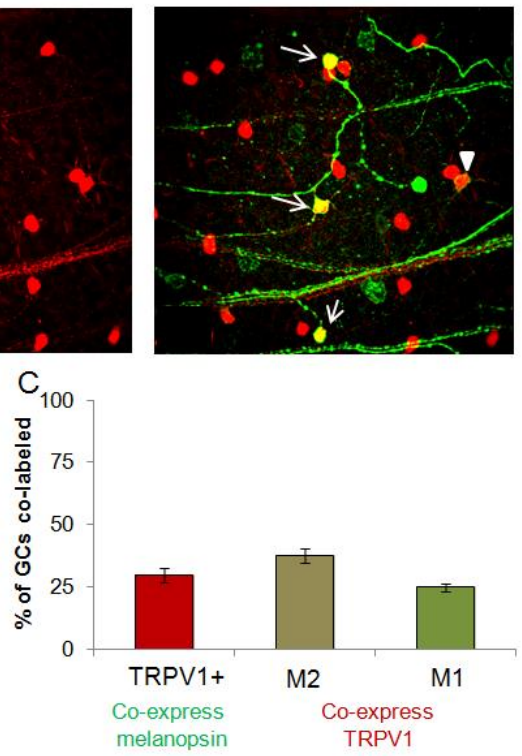

Figure 4.7. Melanopsin is co-expressed in TRPV1+ M1 and M2 GCs (A) Melanopsin label is bright in M1s and dim in M2s. Both bright and dim label is found in TRPV1+ GCs (arrow and arrowhead, respectively). Scale bar is 50 $\mu \mathrm{m}$. (B) The majority of TRPV $1+$ GCs were B3o OFF $(560 \pm 26$ per retina). There were $280( \pm 28)$ TRPV1+ M2 and $168 \pm 56$ TRPV1+ M1 GCs per retina. Finally, WF dACs comprised the smallest population of TRPV1+ cells in the retina ( $56+14$ per retina). (C) Only $30 \%$ of TRPV $1+$ GCs were ipGCs.

Similarly $34 \%$ of M2 and $26 \%$ of M1 GCs were expressed TRPV1.

fluorescence while all other ipGCs are dimmer (Berson et al., 2010; Estevez et al., 2012). Melanopsin was co-expressed in $30 \%$ of TRPV1+ GCs (Fig. 4.7). Of the TRPV1+ cells in the GCL that did not express melanopsin, 95\% (362/382 cells) had larger somas and axons and were presumed to be mostly B3o GCs. 
The extensive co-stratification of the processes from TRPV1+ GCs and ACs prevented morphological identification of them directly. However, the W3 GCs were observed in very small numbers (2 out of 34 melanopsin negative GCs characterized). No tdTomato+ M4 GCs were observed in the TRPV1 ${ }^{\text {cre }} /$ Ai9. Twenty of the TRPV1+ cells in the GCL (5\%) were WF dACs, identified by their very small somas and lack of axon.

The numbers of each GC class were counted and compared based on the presence and brightness of melanopsin label in the four quadrants from three retinas. In a $0.25 \mathrm{~mm}^{2}$ area, there was a mean of three TRPV1+ M1 GCs, five TRPV1+ M2 GCs, ten TRPV1 + B3o GCs and one TRPV1+ WF dACs. Therefore, the density of TRPV1+M1 GCs was $12 / \mathrm{mm}^{2}$, of M2 GCs was $20 / \mathrm{mm}^{2}$, of B3o GCs was $40 / \mathrm{mm}^{2}$ and of TRPV1+ WF dACs was $4 / \mathrm{mm}^{2}$. Given a mouse retinal area of $14 \mathrm{~mm}^{2}$ (Williams \& Goldowitz, 1992; Lyubarsky \& Pugh, 1996), then only 168 out of the reported 640 M1 GCs per retina (Berson et al., 2010) expressed TRPV1 (Fig. 4.7b \& c; 26\%). Similarly, 280 out of 830 M2 GCs per retina expressed TRPV1 (34\%). The majority of TRPV1+ GCs, around 560 per retina, were B3o OFF GCs. TRPV1+ WF dACs were very rare with only 56 per retina. The small numbers of TRPV1+ GCs may be indicative of a very specific function in those cells.

2.5 TRPV1 was expressed in the Brn3b negative subclass of M1 ipGCs

TRPV1+ M1 GCs were a very small population, which may have represented a sub-class of M1. Two M1 subclasses have been identified previously (Chen et al., 2011). I investigated whether TRPV1+ M1 GCs 
represented one of those sub-classes. The axons of two populations of M1 GCs have different central targets. The largest population (approximately $2 / 3$ of M1s; Chen et al., 2011) expresses the transcription factor Brn3b and their axons project to the shell of the olivary pretectal nucleus (OPN). In contrast, the axons of Brn3b negative M1 GCs project to the suprachiasmatic nucleus (SCN; Chen et al., 2011). To determine whether Brn3b positive or negative M1 GCs express TRPV1, I double labeled TRPV1 1 re/Ai9 retinas were with antibodies to melanopsin and Brn3b (Fig. 4.8a). In order to use both antibodies that were made in rabbit, I used a melanopsin antibody conjugated to Alexa 647. Conjugated melanopsin produced weaker label than melanopsin with TSA that produced a signal that could only be seen in M1 GCs. In three retinas, an average of $554 \mathrm{M} 1 \mathrm{GCs}$ were labeled per retina. That number is similar to previous reports of $640 \mathrm{M} 1 \mathrm{GCs}$ in the ganglion cell layer (Berson et al., 2010) and confirms that the majority of M1 GCs were labeled with that technique. Six of the $19 \mathrm{M} 1 \mathrm{GCs}(31 \%)$ I counted in three retinas co-expressed Brn3b (Fig. 4.8). Of those, none of them expressed TRPV1. Similarly, 13 M1 GCs were Brn3b negative and 10 of those GCs expressed TRPV1 (52\%). TRPV1 was expressed in $77 \%$ of the Brn3b negative M1 GCs.

The absence of Brn3b in TRPV1+ M1 GCs is indicative that TRPV1 is expressed in some of the population of M1s that project to the SCN, but the numbers of GCs are low, so I sought to test that hypothesis further. I assessed tdTomato expression in the brains of TRPV $1^{\text {cre }}-\mathrm{AAVmice}$ to determine the targets of TRPV1+ GC axons. Consistent with the absence of Brn3b expression, 
TRPV1+ processes were found terminating in the SCN (Fig. 4.8a). TRPV1+ terminals also were found in the core of the OPN, the target of M2 GCs, but not strongly present in the OPN shell, the target of Brn3b+ M1 GCs (Fig. 4.8b). I compared the spread of tdTomato label in the OPN to that in the TRPV1cre/Ai9

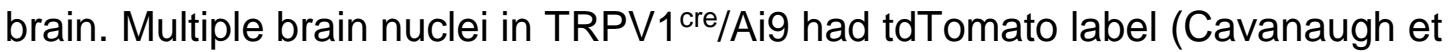
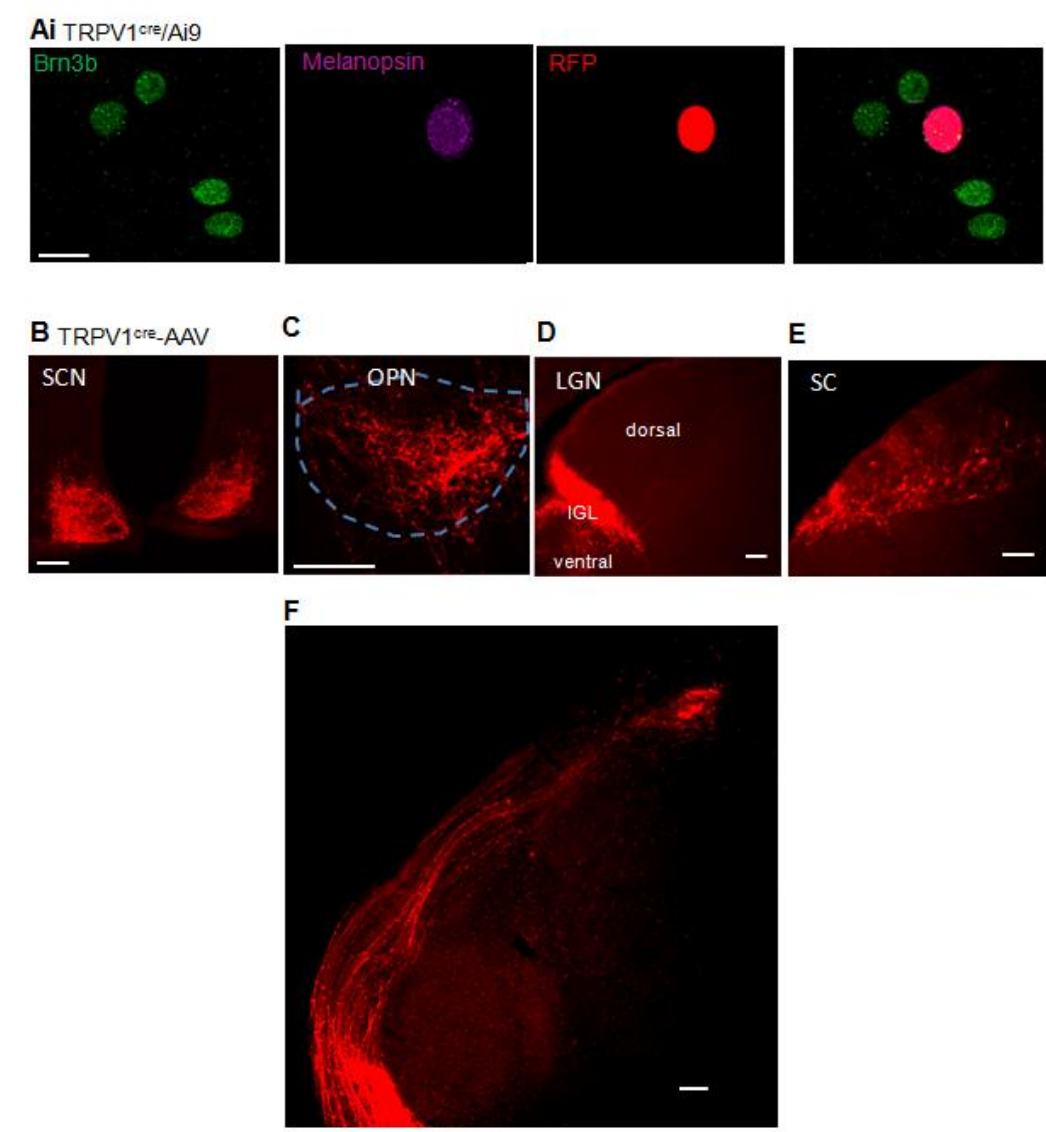

Figure 4.8. TRPV1 is expressed specific subsets of $M 1$ and $M 2$ ipGCs (A) TRPV1 is expressed in M1 GCs, which lack the transcription factor Brn3b. This specific population projects to the SCN (Chen et al., 2011). Consistent with the absence of Brn3b, TRPV1+ M1

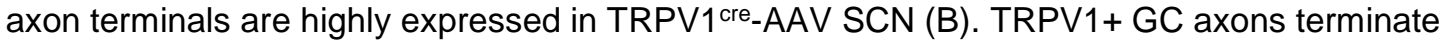
in the core of the OPN (C), IGL, VLGN (D) and SC (E), all know targets of M2 ipGCs. TRPV1+ M2 and B3o GCs do not project to the dLGN (D), suggesting TRPV1 modulates non-image forming vision. (F) TRPV1+ axons send collaterals to both the IGL and OPN, both areas involved in regulation of circadian rhythms. Scale bars are $20 \mu \mathrm{m}$ in A and $100 \mu \mathrm{m}$ in B-F.

al., 2011b) and therefore the origin of tdTomato+ processes could be the brain or retina. I found tdTomato+ processes in the core and shell of the OPN of the 


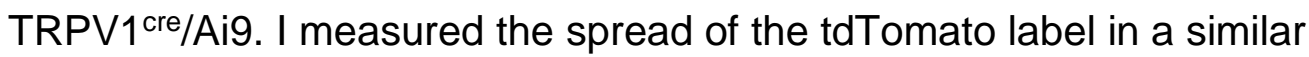
coronal section and compared it to the tdTomato+ GC axons in TRPV1 ${ }^{\text {cre-AAV }}$ (Fig. 4.8c broken outline). The spread of the tdTomato+ processes was smaller and centrally clustered, indicative of GC terminals in the core rather than the shell of the OPN. From that I conclude that TRPV1 was expressed exclusively in Brn3b negative M1 subclass, which projected to the SCN. However, I did not find tdTomato expression in all of the Brn3b negative M1 GCs.

Besides the core of the OPN, M2 GCs project to the dLGN and the SC (Schmidt et al., 2011). The target of B3o GCs is unknown, however the majority of GCs that do not express melanopsin project to the dLGN and SC. M1 axons can target other non-image forming nuclei in the brain. I assessed tdTomato expression other targets areas of GC axons. There were very few tdTomato+ axons that terminated in the dLGN, a target of M2 GCs (Schmidt et al., 2011; Fig. 4.8c). Other known targets of ipGCs include the intergeniculate leaflet (IGL) and ventral LGN (vLGN) (Berson et al., 2003; Chen et al., 2011). The IGL and vLGN

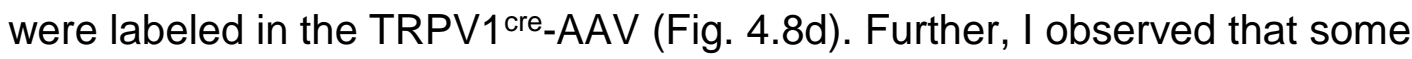
axons which innervated the LGN also innervated the OPN (Fig. 4.8f) and most likely originated from TRPV1+ M2 GCs. The SC was labeled and presumably the post-synaptic target of the TRPV1+ M2 and B3o GCs (Fig. 4.8d; Schmidt et al., 2011).

2.6 Capsaicin induced excitatory currents in amacrine but not ganglion cells

In order to understand the mechanism by which TRPV1 alters GC spiking responses, it is important to establish how it affects the visual responses of the 
cells that express TRPV1. TRPV1 is a non-specific cation channel and bath application of capsaicin leads to $\left[\mathrm{Ca}^{2+}\right]$ elevations in dissociated GCs and microglia (Ryskamp et al., 2014), but does not appear to affect photoreceptors or Müller cells (Sappington et al., 2009). To determine TRPV1 protein expression, tdTomato+ cells in TRPV1 ${ }^{\text {cre }} / \mathrm{Ai9}$ retinas were targeted for whole cell patch clamp recordings in a wholemount preparation. Capsaicin activates TRPM1 in ON bipolar cells and induces excitatory currents, which will result in increased excitatory currents in ON GCs (Shen et al., 2009; Ray et al., 2014). As a result, when I bath applied capsaicin, I isolated excitatory currents mediated only by TRPV1 in GCs in the presence of glutamate receptor blockers (DAP-5, CNQX and L-AP4).

In dissociated GCs, the increased $\mathrm{Ca}^{2+}$ responses induced by capsaicin ( 5 mins; $40 \mu \mathrm{M}$ ) had a transient peak lasting approximately one min, which was followed by a steady fluorescence that remained slightly elevated above baseline for $>7$ min (Ryskamp et al., 2014). The capsaicin signal was absent from TrpV1/GCs, indicative of a TRPV1 mediated $\mathrm{Ca}^{2+}$ response. To determine whether capsaicin could mediate currents in GCs, I bath applied $30 \mu \mathrm{M}$ capsaicin for three min and recorded current responses in whole cell patch clamp configuration $\left(V_{\text {hold }}=-60 \mathrm{mV}\right)$. TdTomato + somas from TRPV1 ${ }^{\text {cre }} /$ Ai9 retinas were targeted using brief fluorescent illumination. To determine the general variability of current fluctuations in GCs, I recorded eight GCs in the absence of capsaicin (Fig. 4.9; see methods). GCs were filled with Lucifer Yellow and Neurobiotin for morphological identification. Five of the eight control GCs were tdTomato+: $1 \mathrm{M} 1$, 


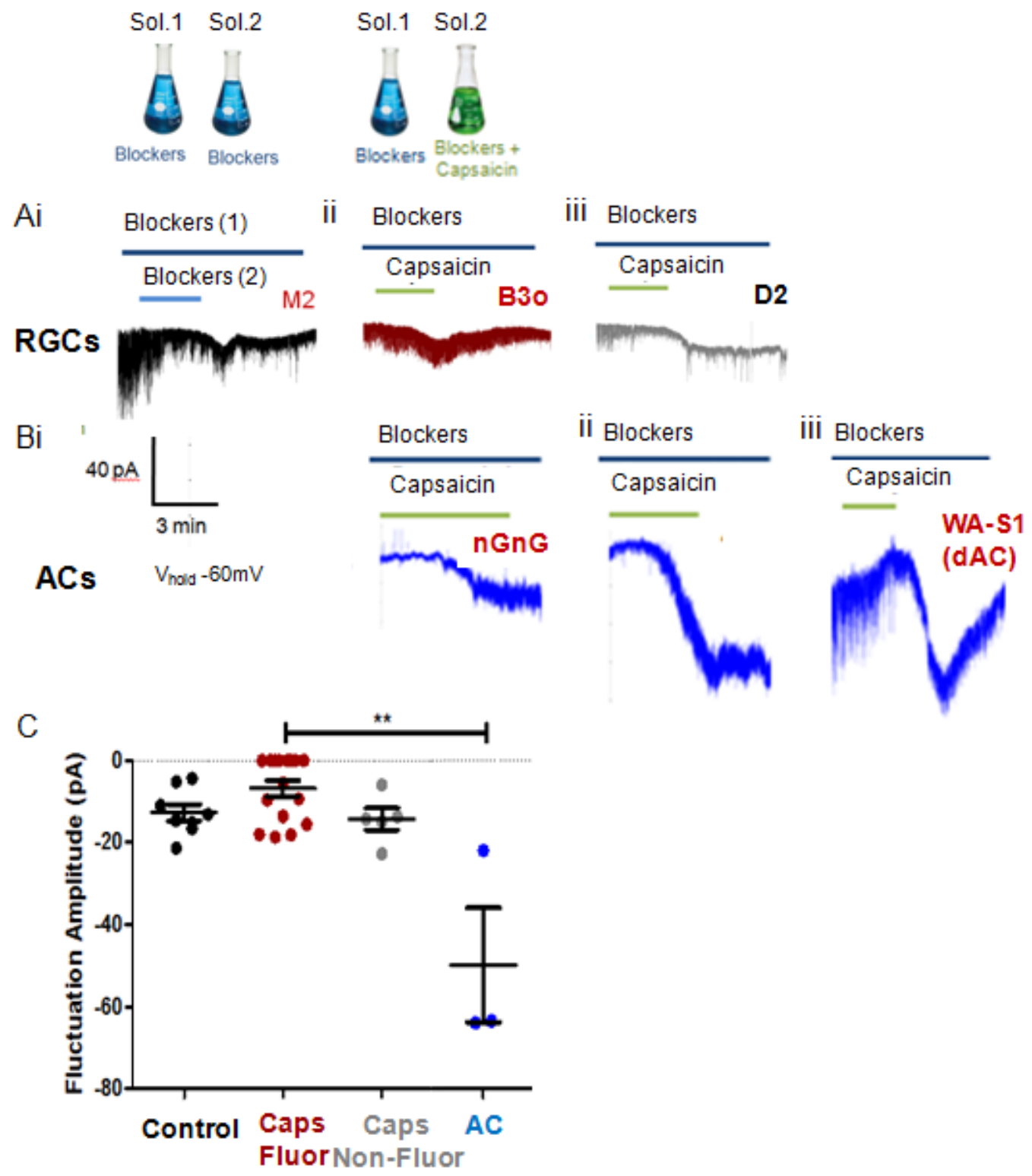

Figure 4.9. TRPV1 agonist capsaicin evokes current in TRPV1+ ACs but not GCs in wholemount retina (Ai) Control experiments measured fluctuations in membrane current in presence of glutamate receptor blockers from two separate solutions. Eight tdTomato+ GCs had mean fluctuation amplitude $-12.6 \pm 2 \mathrm{pA}(\mathrm{C})$. Currents responses to capsaicin were then measured in 16 tdTomato+ GCs under identical conditions. The amplitudes of the currents in the presence of capsaicin were similar to control ( $-6.5 \pm 1.8 \mathrm{pA}$; Aii \& C). Capsaicin responses were measured in 4 non-fluorescent GCs and fluctuations were similar to control $(-14.3 \pm 2.7 p A$; Aiii). (B) Currents responses to capsaicin were then measured in 3 tdTomato+ ACs under identical conditions. The amplitudes of the currents in the presence of capsaicin were larger than control ( $-49.8 \pm 13.9 \mathrm{pA} ; \mathrm{p}=0.006$; $\mathrm{B} \& \mathrm{C}$ ). One $\mathrm{AC}$ had a displaced soma (iii) Capsaicin evoked TRPV1 mediate currents in ACs but not in the retinal portion of GCs. 
$1 \mathrm{M} 2$, and 3 B3o. Three were non-fluorescent D2 bistratified GCs (Sun et al., 2002) whose processes stratified with the CHAT bands. The responses from all control GCs were used to set a minimum criterion for a capsaicin evoked response, e.g., the amplitude in the presence of capsaicin had to be statistically larger than control membrane fluctuations (Fig. 4.9ai). Responses from 16

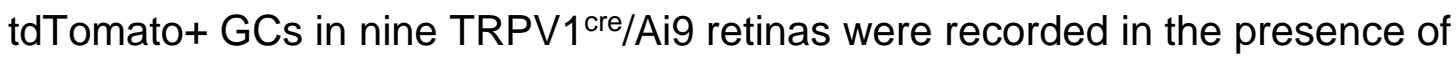
capsaicin. Eight were B3o, five were M1, and three were M2. Capsaicin responses from five non-fluorescent $\mathrm{D} 2$ bistratified, one $\mathrm{A} 1 \mathrm{ON} \mathrm{GC}$, and two unknown GCs also were recorded. Fig. 4.9 shows representative traces of membrane current fluctuations $\left(\mathrm{V}_{\text {hold }}=-60 \mathrm{mV}\right)$ from control recordings and recordings in the presence of capsaicin. All of the fluctuations in response to capsaicin fell within the range of the control fluctuations $(0$ to $-21 p A ; p=0.58)$. I determined that capsaicin did not evoke a current response in any GCs. In light of known TRPV1 expression and function in other neural systems, there were several possible explanations for absence of capsaicin currents in TRPV1+ GCs in wholemount retina, which I will mention briefly here and expanded on in Chapter VI (General Discussion and Future Directions).

1. TRPV1 could be present outside the retina, such as in GC axon terminals.

2. TRPV1 may also be located intracellularly and regulating calcium stores there rather than passing membrane current.

3. TRPV1 in vesicle stores within the GC may be quickly inserted in the membrane and pass calcium current in response to noxious stimuli, such 
as dissociation of the GCs in culture. This process occurs within minutes of noxious stimuli and increases excitatory currents in affected cells.

To determine if TRPV1 can mediate current at all, Dr. Borghuis and I recorded from tdTomato+ ACs $(n=2)$ in wholemount retinas. Again, capsaicin $(30 \mu \mathrm{M})$ was bath applied for seven or five minutes. In contrast to the GCs, capsaicin evoked a large inward current in those ACs (Fig. 4.9b; -22pA \& -65 pA, respectively). The responses reached a plateau approximately seven min after onset of capsaicin application. We monitored the ACs for 40 and 20 min after capsaicin application and the current response did not return to baseline. The recorded ACs were imaged using two-photon microscopy directly after recording and morphologically identified. The morphology of one AC was an nGnG, consistent with morphological analysis of TRPV1+ ACs. The morphology of the second $A C$ was undetermined due to insufficient filling of the $A C$ by the Alexa dye.

While targeting TRPV1+ GCs, I inadvertently recorded from a tdTomato+

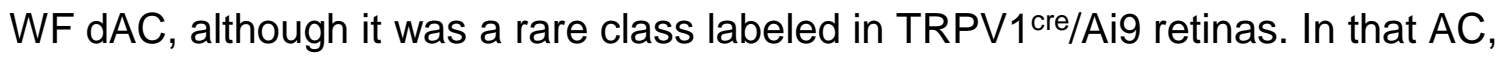
three min capsaicin application evoked a large inward current, similar to the other ACs (Fig. 4.9biii; -64pA). The current had a transient peak that reached a maximum approximately 7 min after capsaicin application. The current declined and returned to baseline holding $15 \mathrm{~min}$ after capsaicin application. Although the number of ACs recorded from was small, the similarity among the tdTomato+ ACs and dACs lead to the conclusion that they expressed TRPV1 channels that 
can be directly gated by capsaicin and pass current when activated. Possible TRPV1 ligands that are endogenous in the retina are discussed in the next section.

\section{Discussion}

3.1 Capsaicin induced excitatory currents in amacrine but not ganglion cells

To determine the specific cell types that express TRPV1 in the retina, I used a TRPV $1^{\text {cre }}$ knockin mouse (Cavanaugh et al., 2011a\&b) and induced tdTomato expression in TRPV1+ cells using two methods: TRPV1 ${ }^{\text {cre }} /$ Ai9 to assess developmental and mature expression and TRPV1 ${ }^{\text {cre }}$-AAV to assess mature expression only. The data between the two reporter types is largely consistent, therefore TrpV1 continues to be transcribed in ACs and GCs in the mature mouse. TRPV1 is expressed in two NF ACs and one WF dAC, which have been previously identified in the rabbit (MacNeil et al.,1999) and mouse (Pérez De Sevilla Müller et al., 2007; Badea \& Nathans, 2004; Lin \& Masland, 2006; Kay et al., 2012; Lee et al., 2014; Zhu et al., 2014). That expression is consistent with reports by Leonelli and colleagues (2010\& 2013) in which they found TRPV1-ir in NF ACs in the rat. I did not find consistent TRPV1-ir in the mouse retina, which I attribute to low specificity of the TRPV1 antibody to mouse protein. TRPV1 protein expression in the mouse DRG is $20-30$ fold higher than the retina and TRPV1-ir requires signal amplification to accurately assess TRPV1 localization there. The faint punctate like label in the IPL of the mouse retina then 
is not surprising and is too faint to be useful for determining TRPV1 expression patterns. However, capsaicin evoked currents confirmed TRPV1 protein expression in three tdTomato+ ACs. The prospective role of TRPV1 in each AC type is discussed below:

1. The Flag A AC stratifies in s 2-3 and express glycine (MacNeil et al.,1999). Activation of TRPV1 increases intracellular calcium by entry through the channel or TRPV1 mediated calcium regulation of intracellular stores (Olah et al., 2001; Liu et al., 2005; Karai et al., 2004; Medvedeva et al., 2009). Subsequently, TRPV1 commonly plays a role in sustained, asynchronous neurotransmitter release. TRPV1 in the Flag A ACs may modulate light responses by regulating tonic glycine release.

2. The $n G n G$ stratifies in $s 1-3$ and do not express glycine or GABA as previously reported (Kay et al., 2012). The neurotransmitter released by the nGnG ACs has not been determined (Kay et al., 2012). The expression of TRPV1 in these ACs gives prospective targets to investigate. TRPV1 is activated by endocannabinoids and endovanilloids such as anandamide, 12-Hepete, and NADA (see Ryskamp et al., 2014 for a review). Endovanilloids are synthesized from membrane phospholipids and cannot be immuno-labeled directly, but anandamide regulating enzymes have been localized to ACs in the goldfish and rat retina (Zimov \& Yazulla, 2007; Hu et al., 2010; Zabouri et al., 2011). Interestingly, ACs expressing the regulatory enzyme 
FAAH in the goldfish retina co-localize with TRPV1-ir. Future studies are needed to determine if endocannabinoid related enzymes are expressed in mouse TRPV1+ nGnG ACs.

3. The TRPV1+ WF dAC resembles the conventionally placed WA S3 (Pérez De Sevilla Müller et al., 2007), and bifid (Badea \& Nathans, 2004), but are most similar to the displaced nNOS-2 WF AC (Zhu et al., 2014). The expression of nNOS in TRPV1+ AC has yet to be determined. Interestingly, Leonelli and colleagues (2013) report TRPV1 + ACs co-stratify with NF nNOS+ ACs in the rat and TRPV1 activation may regulate $\mathrm{nNOS}$ expression and NO signaling in the retina. They suggest that NO upregulation by TRPV1 activation by capsaicin induced death of GCs. The specificity of TRPV1 (rather than TRPV1-independent capsaicin effects) to induce NO release still needs to be confirmed in $\operatorname{TrpV} 1 \%$. The idea that TRPV1 may regulate intracellular $\mathrm{Ca}^{2+}$ concentrations in nNOS WF ACs and subsequent NO synthesis and release, $\mathrm{a} \mathrm{Ca}^{2+}$ dependent process, may be a target for future investigation.

All AC types co-stratify with glutamatergic ACs but do not express vGlut3. The positions of the processes give them the potential to form synaptic connections with each other or the GACs. Peters' rule states that neuronal processes connect in proportion to their proximity and dendritic or axonal density (Peters \& Feldman, 1976; Peters \& Payne, 1993). However synaptic connection between ACs and GCs that colocalize s 2-3 violate Peter's rule. Krishnaswamy 
and colleagues (2015) found that besides co-stratification, recognition molecule sidekick 2 in GACs and post-synaptic W3B GCs enhanced pairing between the specific cells. GACs and other ACs and GCs stratifying in s 3 play roles in visual processing of image motion, local edge detection and local contrast change (Chiao and Masland, 2003; Murphy and Rieke, 2008; Zhang et al., 2012;

Krishnaswamy et al., 2015, Lee et al., 2014 \& 2016). GACs enhance extraction of specific features by these cells (Kim et al., 2015). Co-stratification of TRPV1+ ACs and GCs with GACs and related neurons suggest TRPV1 may play a role in some or all of these processes.

\subsection{TRPV1 in ganglion cells likely regulates non-image forming visual signaling in the brain}

There are previous reports of TRPV1 in GCs in mouse (Sappington et al., 2009 \& 2016, rat (Sappington et al., 2009; Leonelli et al., 2009 \& 2014), human, monkey (Sappington et al., 2016) and bovine retinas (OLeary et al., 2014). Most studies of TRPV1 in the retina show expression of TRPV1 in GCs in rats and mice, however the numbers of labeled cells are variable between studies. Leonelli and colleagues (2009) found TRPV1-ir in a small number of GCs in the rat, while the Calkins group reports TRPV1-ir in nearly all GCs in the mouse retina. The concentration of the antibody used in the latter studies is 1:50. My data shows that the same antibody at the same concentration results in high nonspecific label in the DRG and retina of WT and TrpV1/- mice. In general, the TRPV1 antibodies were not very useful in mice. Sappington and colleagues also 
report TRPV1 mRNA expression in 70\% of GCs using in situ hybridization with high inter-retinal variability. My results from TRPV1 reporter mice determined that TRPV1 is expressed only in 6\% of GCs, consistent with what Leonelli and colleagues found in the rat (2009). I further determined that TRPV1 is expressed in small populations of specific GC classes. The GC classes labeled in TRPV1 $1^{\text {cre }}$ mice have been previously identified (Sun et al., 2002; Berson et al., 2010; Farrow et al., 2013). Capsaicin application in the wholemount does not evoke current in TRPV1+ GCs, suggesting TRPV1 may reside in the axons or terminals of these cells, or may play a role in intracellular regulation of $\mathrm{Ca}^{2+}$. The prospective role of TRPV1 in each AC type is discussed below:

1. The B3o GC (Sun et al., 2002) has a medium sized dendritic area, which stratifies in s3 of the IPL. It resembles the PV-4 (Farrow et al., 2013) in the parvalbumin cre mice. It may also resemble the W7 GCs (Kim et al., 2010). However the TRPV1+ OFF GC may have a smaller dendritic area than the latter. GCs with W3 morphology were labeled less frequently in both reporter types. Interestingly, very few, if any axons from the TRPV1 ${ }^{\text {cre }}$-AAV mice terminated in the dLGN, the target of most image forming GCs, but tdTomato+ axons were observed in the SC. Functionally the $\mathrm{SC}$ integrates visual and other sensory information for directing head and eye movements (May, 2006; Krauzlis et al., 2013). The specific function of B3o GCs in visual processing is unknown. Maione and colleagues (2009) report direct evidence for TRPV1 expression and function in GC axon terminals in the SC. Expression there is higher in the 
developing retina and is necessary for long-term depression, which is likely involved in dendritic pruning. The TRPV1 antagonist iodoresinaferatoxin blocked long term depression, however trials with $\operatorname{TrpV} 1 \%$ mice were not reported. TRPV1 is present but decreased in the adult axon terminals and has a different function (long-term depression is not observed in the adult SC).

2. A small population of $M 1$ ipGCs ( $30 \%)$ expresses TRPV1 and is morphologically similar to previous reports. Intrinsically photosensitive GCs are $1 \%$ of all GCs (Berson et al., 2010) and only 30\% of all ipGCs express TRPV1. The small numbers of TRPV1+ipGCs may be indicative of a very specific function in these cells. GC classes extract distinct pieces of information to send to post-synaptic targets in the brain, where signals are integrated (Masland, 2012). The specific site of axon termination is related to the function of those GCs. Among the M1 ipGCs, those that express Brn3b project to the shell of the OPN and regulate pupil constriction in response to light. The Brn3b negative M1s project to the SCN and IGL regulate photoentrainment (Güler et al., 2008; Hatori et al., 2008, Ecker et al., 2010; Chen et al., 2011). My results indicate the TRPV1+ M1s represent the latter population. In the absence of light input, the intrinsic periodicity of the circadian rhythms is not exactly $24 \mathrm{hrs}$. Light activated M1 input synchronizes the circadian clock to the day/night environment (see Hatori \& Panda, 2010 for a review). Photoentrainment of the circadian clock has not been studied in $\operatorname{TrpV} 1 \%$ mice and would be an 
interesting target of future studies. Interestingly, endocannabinoid serum expression is under circadian regulation. Also, endocannabinoids reside in the SCN and have been shown to modulate photoentrainment of the circadian rhythm. In that study, photoentrainment was mediated by CB1 receptors on GABAergic interneurons and TRPV1 agonists were not tested.

3. M2 ipGCs project to the core of the OPN and may also send axons to the dLGN, vLGN, and SC (Baver et al., 2008; Ecker et al., 2010; Schmidt et al., 2011; Estevez et al., 2012; Hughes et al., 2016). TRPV1+ M2s send projections to the LGN and core of the OPN. TdTomato+ axon terminals were also observed in the SC. The absence of tdTomato+ terminals in the dLGN suggests the TRPV1+ M2s (and other TRPV1+ GCs), have functions related to non-image forming processing. In contrast to M1 GCs, the majority of the light evoked response is mediated by photoreceptor input to the M2 rather than melanopsin activation. The function of M2 GCs is still under investigation, but may be involved in circadian regulation of blood circulation or intraocular pressure (Blasiak et al., 2013) and has recently been implicated in light induced hypersensitivity to pain or light aversion in rats (Martenson et al., 2016). Pain modulating neurons in the rostral ventromedial medulla receive light information mediated through the OPN. That pathway has been implicated as a prospective source of light induced hyperalgesia in conditions such as migraine. It would be 
interesting to know if inhibiting TRPV1 function could modulate pain inducing light information there.

\section{Conclusions}

In this study I used TRPV1 reporter mice to determine the specific retinal neurons that express TRPV1. I determined that TRPV1 is localized to two NF AC classes. There, TRPV1 may regulate release of glycine and modulate visual signals to GCs. The presence of TRPV1 in ACs with unknown neurotransmitter and WF dACs, which may also express nNOS have potential to the help elucidate the role these cells play in shaping vision. Three main GC classes were consistently labeled in the mature mouse retina. TRPV1 in GCs that regulate circadian rhythms in combination with a likely endocannabinoid activator make TRPV1 a target of study in a field with important implications in health and disease. Further, the selective label of subsets of ipGCs make the TRPV1 reporter mouse a useful tool for clarifying the function of the pathways they innervate. 


\section{CHAPTER V}

\section{LRIT3 REGULATES TRPM1 LOCALIZATION AND OPL SYNAPTIC STRUCTURE AND FUNCTION}

\section{Introduction}

Transient receptor potential cation channel subfamily M member 1 (TRPM1) is a non-selective cation channel whose function in the retina has been well described (Bellone et al., 2008; Morgans et al., 2009; Shen et al., 2009; Koike et al., 2010). Bellone and colleagues first described a potential role for TRPM1 in retinal signaling when they observed mutations in TRPM1 were associated with night blindness and also coat spotting in Appaloosa horses (Bellone et al., 2008). Subsequent work has confirmed that TRPM1 is expressed in the dendritic tips of ON BCs and is required for depolarization of ON BCs to light (Koike et al., 2010; Morgans et al., 2009; Nakamura et al., 2010; Pearring et al., 2011). Absence of TRPM1 or components necessary for the function of TRPM1 results in the human disorder, complete congenital stationary night blindness (cCSNB; Miyake et al., 1987; Audo et al., 2009; Zeitz et al., 2015), which is characterized by decreased vision in low light. Other visual abnormalities that have been associated with cCSNB patients are: decreased visual acuity, myopia, nystagmus and strabismus 
(Carr, 1974; Boycott et al., 1993; Zeitz et al., 2015). ON BC dysfunction in cCSNB leads to a characteristic phenotype in which the ERG has a normal a-wave although the b-wave is absent (Bornschein, 1952; Miyake et al., 1986).

Components of the ON BC signaling cascade associated with TRPM1 function have been identified due to mutations in humans (Dryja et al., 2005; Pinto et al., 2007; Audo et al., 2009; Zeitz et al., 2015), horses (Bellone et al., 2008), and/or mice (Masu et al., 1995; McCall \& Gregg, 2008) which lead to the nob phenotype. Responses through the ON pathway are initiated by sign inverting synapses between rod and cone photoreceptors with ON BCs. TRPM1 is associated with the metabotropic glutamate receptor mGluR6 and G-proteins Gao and Gßy. They and multiple accessory proteins form a signaling complex (signalplex) in ON BCs, which mediates transmission of visual signal at the synapse. The G-protein cascade, described in detail in the introduction (Fig. 1.4), is summarized here. In the dark, glutamate is continuously released from rod and cone photoreceptors and binds to the mGluR6 receptors on the post-synaptic ON BC dendrites (Nakajima et al., 1993; Nomura et al., 1994). Once bound, a G。 gprotein coupled cascade is activated (Nawy et al., 1999; Dhingra et al., 2000, 2002, 2012), which, through several intervening steps that have yet to be fully elucidated, closes the TRPM1 cation channel (Morgans et al., 2009; Shen et al., 2009; Koike et al., 2010; Peachey et al. 2012). In response to light, glutamate release is decreased and mGluR6 is inactivated. The regulator proteins G $\beta 5$, RGS7 and RGS11 (Morgans et al., 2009; Jeffrey et al., 2010; Cao et al., 2012), as well as GPR179 and R9AP (Peachey et al., 2012; Ray et al., 2014), mediate the rapid 
inactivation of the mGluR6 cascade and opening of the TRPM1 channel. Influx of $\mathrm{Na}^{+}$and $\mathrm{Ca}^{2+}$ through the non-specific TRPM1 cation channel depolarizes the ON BC (Shen et al., 2009; Morgans et al. 2009). Accessory proteins are critical for the proper localization and function of the mGluR6 ON BC signalplex. The leucine rich repeat protein, nyctalopin, is required for TRPM1 localization in the synaptic membrane (Gregg et al., 2003; Pearring et al., 2011). Mice with mutations in the Nyx gene encoding nyctalopin (Nyx $\left.{ }^{\text {nob }}\right)$ have the no b-wave (nob) ERG phenotype (Pardue et al., 1998; Gregg et al., 2003; McCall et al., 2008). Recent studies have shown that replacing absent components of the cascade with functional protein can restore ON pathway signal in a mouse model of the disorder (Gregg et al., 2007; Scalabrino et al., 2015).

A similar accessory protein, leucine-rich repeat immunoglobulin-like transmembrane domain 3 (LRIT3), also is absent in some cCSNB patients (Zeitz et al., 2013) and a mouse with a nob ERG (Lrit3nob6; Neuillé et al., 2014). Similar to in the nyctalopin knockout, the absence of LRIT3 results in the mislocalization of TRPM1 (Neuillé et al., 2015). However, Lrit3nob6 mice display a phenotype that differs from Nyx ${ }^{\text {nob; }}$ mGluR6, GPR179, RGS7, RGS11 and Gß5 is absent from the dendritic tips of the ON cone BCs (CBCs), but present in RBCs. In addition, expression of the peanut agglutinin (PNA) binding glycoprotein at the cone terminals also is downregulated (Neuillé et al., 2015).

To better understand the role of LRIT3, we created a mouse with a targeted mutation in exon two of the Lrit3 gene, the coding region for the leucine rich repeat sequence using zinc-finger nuclease technology. We determined that expression 


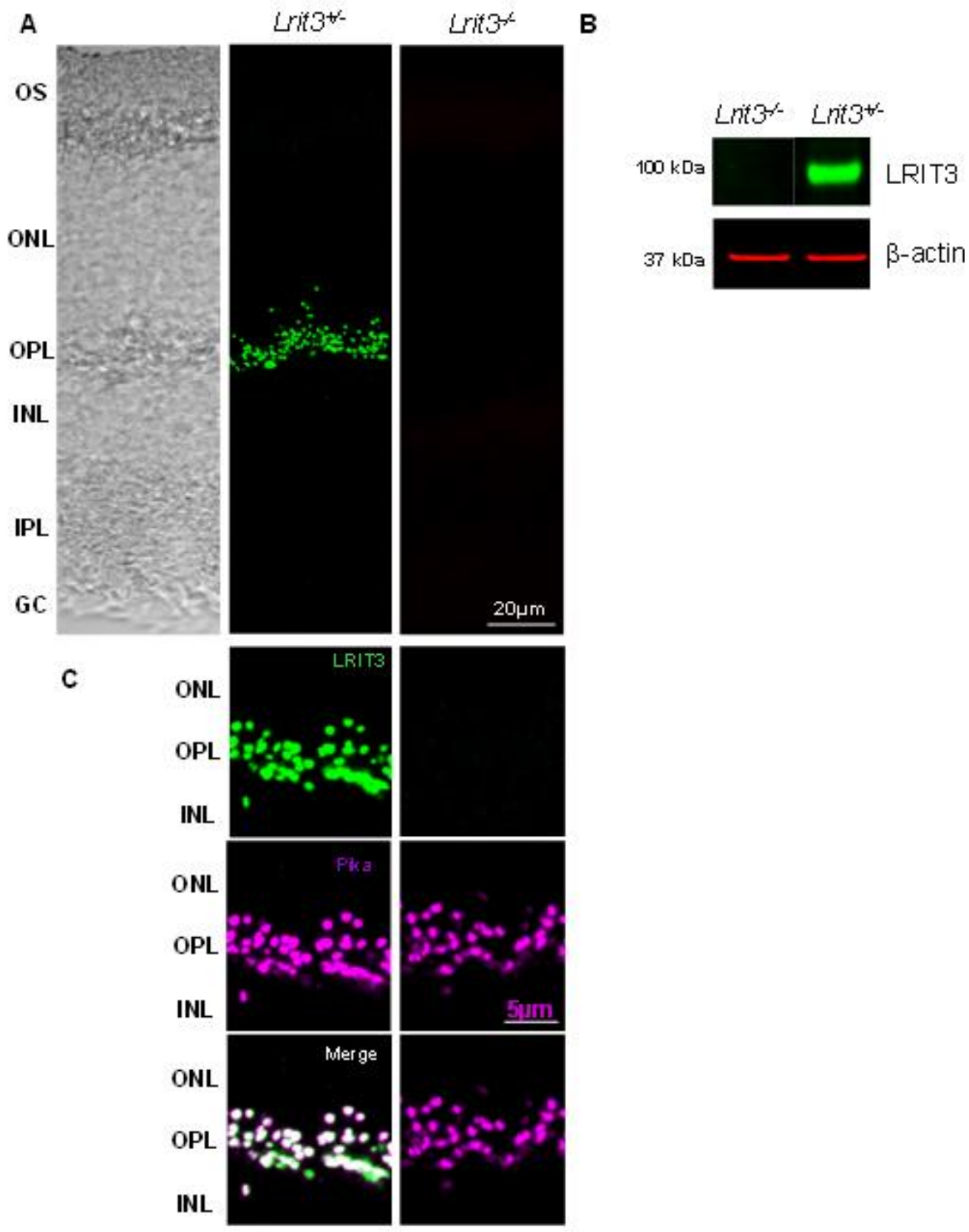

Figure 5.1. Immunohistochemistry and Western blots verify LRIT3 expression is eliminated in the $\mathrm{Lrit3}^{-/}$mouse retina A) Vertical sections of $\mathrm{Lrit}^{+/-}$and $\mathrm{Lrit3}^{+/}$retinas were labeled with antibodies to LRIT3. The absence of LRIT3 label in the Lrit3-- confirms the specificity of the antibody. B) Similarly, Western blot data reveals an absence of LRIT3 protein in the Lrit3 $^{+-}$retina. C) Vertical sections were co-labeled with antibodies to LRIT3 and Pikachurin, which is present at the dendritic tips of ON BCs. LRIT3 is colocalized with Pikachurin in the Lrit3 $^{+/}$on both the RBC puncta and the congregation of protein representing the ON CBCs. Pikachurin, but not LRIT3 is present in the Lrit3\%- 
of LRIT3 protein was absent in these Lrit3\% mice and I showed that they display a nob ERG phenotype. We further examined the structure of the OPL and assessed visual function through the ON and OFF pathways in the in Lrit3\% mice. We determined that LRIT3 is required for nyctalopin expression and that in the

A
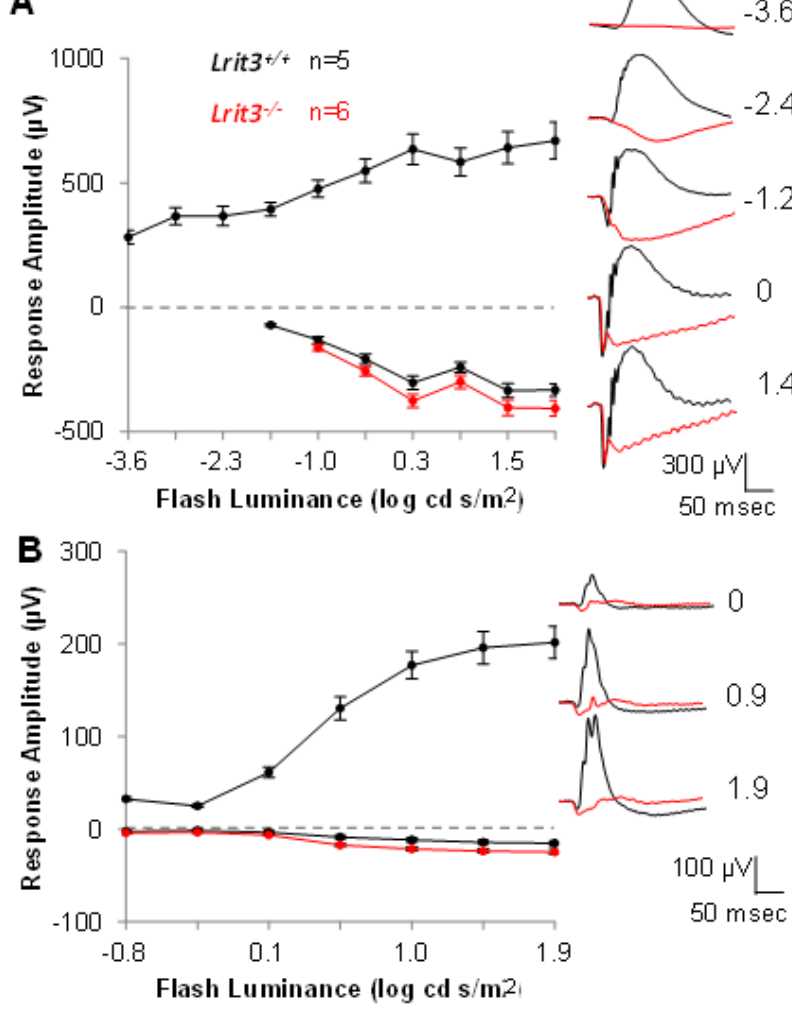

Figure 5.2. ERGs from $\mathrm{Lrit3}^{-/-}$mice express a no bwave phenotype consistent with models of cCSNB A) Scotopic ERGs from Lrit3 ${ }^{+/}(\mathrm{n}=6)$ and Lrit3 ${ }^{-/}(\mathrm{n}=6)$ have similar amplitude a-waves, however the Lrit3-/lacks a b-wave. B) Photopic ERGs from the same mice have similar amplitude a-waves, however the Lrit3 $^{-1}$ lacks a b-wave. Results are consistent with normal photoreceptor function, but an absence of signal through the rod and cone ON BCs. absence of LRIT3 and nyctalopin, TRPM1 is mislocalized in ON BCs. Further, visual signal transduction through the OFF pathway in Lrit3 ${ }^{-/}$is significantly lower than Lrit3 $^{+/+}$and other nob mice. We determined LRIT3 is required for normal glutamate transmission from cones to OFF BCs. This alteration in glutamate signal, in combination with altered synaptic protein expression in

Lrit3 $^{--}$, suggest a trans-synaptic functional role for LRIT3 at the cone terminal. 


\section{Results}

\subsection{LRIT3 is absent in the Lrit3 ${ }^{\text {emrgg1 }}$ mouse retina}

Using the ERG, LRIT3 has been shown to be critical to ON BC function in. We designed zinc finger nucleases targeted to a highly conserved domain in Lrit3 exon 2 (see section 2.1.2 Generation of Lrit3 Zinc Finger Nuclease (ZFN) knockout mice). Of the several Lrit3 mutant lines identified (manuscript in preparation), one had a 40 base pair deletion (Lrit3emrgg1) and it is used in the results of our studies presented here. We also generated an antibody to LRIT3 to use in immunohistochemistry and western blotting.

\subsubsection{Generation of Anti-LRIT3 antibody).}

Immunohistochemistry (Fig. 5.1a) on transverse retinal sections show that LRIT3 is expressed exclusively in a punctate pattern in the OPL in the Lrit3+/retina. This expression is absent in Lrit3-- retina. Western blots (Fig. 5.1b) verify the elimination of LRIT3 protein expression. LRIT3 is co-localized with Pikachurin, the extracellular matrix protein that resides in the synapse between photoreceptors and ON BCs (Fig. 5.1c). The expression of LRIT3 indicates it is part of the ON BC signalplex, which is consistent with previous reports in the Lrit3nob6 using different dendritic markers (Neuillé et al., 2015).

2.2 Glutamate Receptors Are Functional in OFF but not ON BCs in the Lrit3 $^{-/-}$ 
I confirmed that this Lrit3 $^{-}$model lacked ON BC function using the electroretinogram (ERG). The b-wave is absent in the Lrit3 $^{-\%} \mathrm{ERG}$ responses to dim flashes indicating RBCs do not depolarize to light in Lrit3\%- retinas. The a-

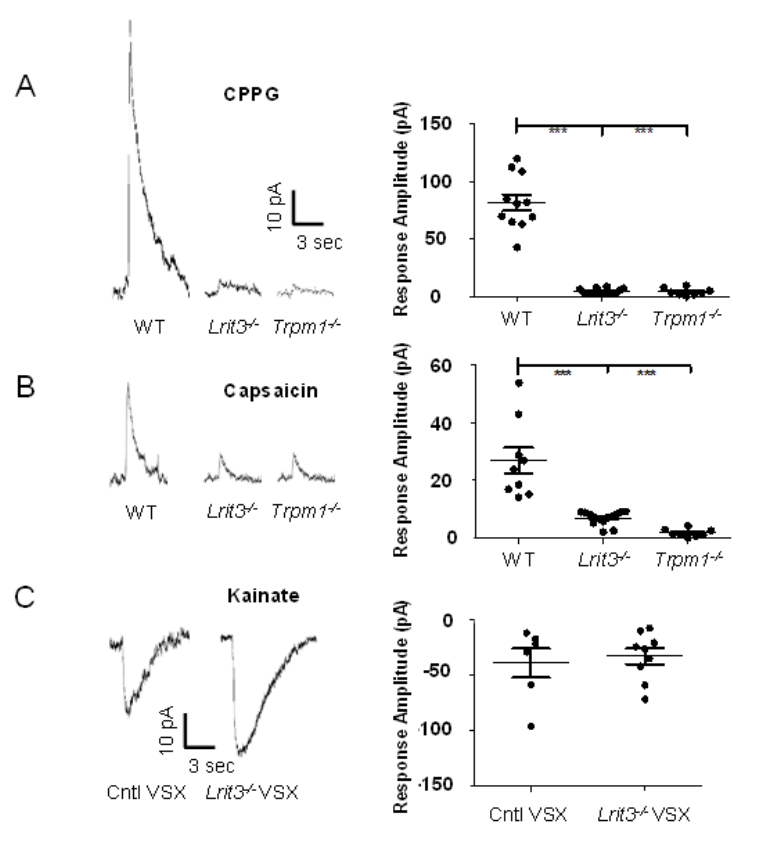

Figure 5.3. Responses of BCs to CPPG, Capsaisin, or Kainate puffs A) Representative voltage-clamp responses ( $\mathrm{V}_{\text {hold }}=+50 \mathrm{mV}$ ) of $\mathrm{WT}$, Lrit3 $^{-1}$, and Trpm 1\%RBCs evoked by puff application of the mGluR6 antagonist CPPG (0.6 $\mathrm{mM}, 200 \mathrm{msec}$ ) delivered with the mGluR6 agonist L-AP4 $(4 \mu \mathrm{M})$ in the bath solution. Current responses to CPPG in Lrit3\% and Trpm1- RBCs were smaller than WT $(p<0.001)$ and did not differ from each other $(p>0.05)$. B) Representative voltage-clamp current responses of WT, Lrit3 ${ }^{--}$, and Trpm 1\% RBCs evoked by puff application of the TRPM1 agonist capsaicin (10 $\mu \mathrm{M}, 200 \mathrm{msec}$ ). Current responses to Capsaicin in Lrit3\% and Trpm 1\% RBCs were smaller than WT $(p<0.001)$ and did not differ from each other $(p>0.05)$. C) Fluoresently labeled Type 1 and 2 OFF BCs were targeted for patch clamp recording in VSX1 and Lrit3--/VSX1 retinas.

Representative voltage-clamp current responses $\left(\mathrm{V}_{\text {hold }}=-60 \mathrm{mV}\right)$ of VSX1 and Lrit3--/VSX1 OFF BCs evoked by puff application of Kainate $(4 \mu \mathrm{M}$, $200 \mathrm{msec}$ ). Response amplitudes were similar between VSX1 and Lrit3--/VSX1 ( $>$ > 0.05). wave amplitude in the Lrit3--

ERG responses to the same

stimuli is similar to $\mathrm{Lrit3}^{+/}$.

Photoreceptors in Lrit3-- $^{-}$

retinas hyperpolarize to light

(Fig. 5.2a). Similarly, the

individual responses from the

Lrit3\%- mice to a family of

flashes above a light

background (20 cd/m2)

lacked an ON BC driven b-

wave, while the cone driven

a-wave also was normal (Fig.

5.2b). A no b-wave ERG

phenotype is emblematic of

retinas that lack functional

ON BCs (Peachey et al., 2007; McCall \& Gregg, 2008) and our results show that the 
Lrit3\%- mouse is similar to the Lrit3nob6 mouse (Neuille et al., 2014).

To directly determine if the ON BC signalplex was functional in the absence of LRIT3, we made whole cell patch clamp recordings of $L$ rit3 ${ }^{-/}$RBCs. In the presence of $4 \mathrm{uM}$ the mGluR6 agonist, L-AP4 $(4 \mu \mathrm{M})$, mGluR6 antagonist CPPG was puffed onto the RBC dendrites to displace L-AP4 bound to mGluR6. This pharmacologically simulates flashes of light (Ray et al., 2014). We also puffed the TRPM1 agonist, capsaicin, to determine if TRPM1 channels could be directly gated. As previously reported (Ray et al., 2014), both pharmacological manipulations resulted in robust outward currents with the rod BCs held at +50 $\mathrm{mV}$ in control rod BCs (Fig. 5.3a \& b). In contrast, only a small response was evoked in Lrit3 ${ }^{-/}$RBCs by either CPPG or capsaicin (Fig. 5.3a \& b). An identical residual response was recorded in Trpm1\% RBCs, consistent with our previous work and likely results from an effect of these drugs on unknown channels (Ray et al., 2014).

We evaluated responses in Types 1 and 2 OFF BCs using Kainate puffs to activate ionotropic glutamate receptors. We targeted OFF BCs in VSX1-CFP reporter and Lrit3--NSX1-CFP retinas (Fig. 5.3c). The amplitudes of the responses to Kainate between control and Lrit3-- OFF BCs was similar $(p>$ 0.05). Together the results show that the mGluR6 signalplex is disrupted in this Lrit3 ${ }^{-/}$retina, whereas as least two subsets of OFF BCs express Kainate glutamate receptors and are responsive to Kainate puffs.

\subsection{LRIT3 is Required for Expression of Nyctalopin}


TgEyfp-Nyc

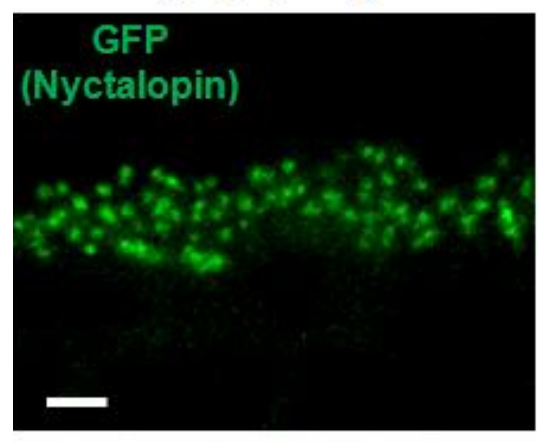

Lrit3 $^{+/+}$

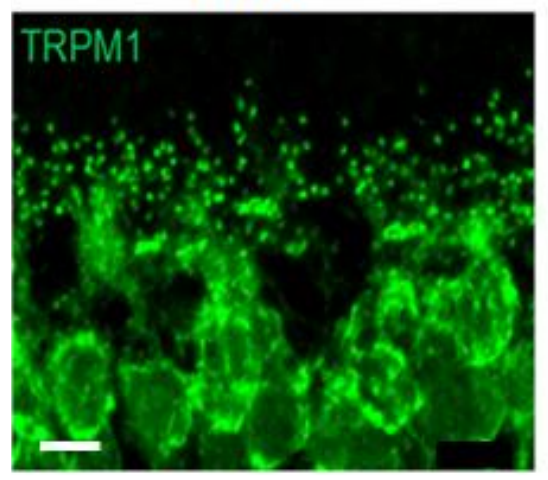

TgEyfp-Nyc/Lrit3-/-

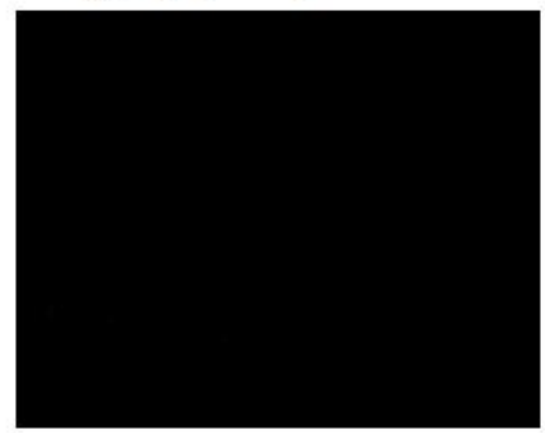

Lrit3 $^{-/}$

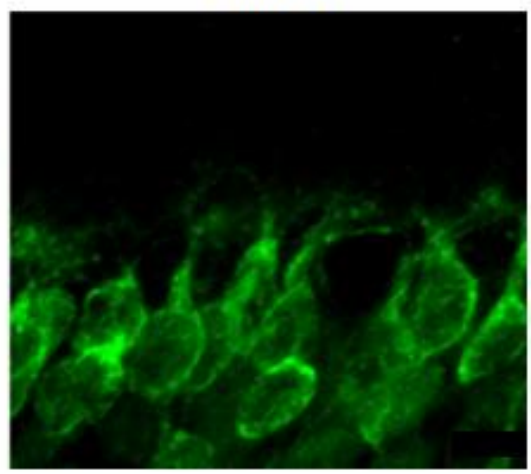

Figure 5.4. Nyctalopin is absent in the Lrit3 ${ }^{-/}$retina A) Vertical slices from TgEyfp-Nyc and TgEyfp-Nyc:Lrit3\% mouse retinas were labeled with antibody to GFP. EYFP-nyctalopin fusion protein is expressed in puncta in the OPL on the tips of the RBCs as well as large clusters representing the tips of the ON CBC terminals. When LRIT3 is absent, the EYFP-nyctalopin fusion protein also is absent. B) TRPM1 is expressed at the dendritic tips of RBCs and ON CBCs. The absence of LRIT3 eliminated the expression of TRPM1 from the dendritic tips of both RBCs and ON CBCs.

It has previously been shown that TRPM1 expression is absent in the OPL of the Lrit3nob6 mouse (Neuillé et al., 2015). The absence of a response to capsaicin puffs is consistent with this interpretation. However, we have previously shown that expression of TRPM1 requires expression of nyctalopin (Pearring et al., 2011) and because the absence of expression can be interpreted to represent a protein:protein interaction, I evaluated whether nyctalopin is expressed in Lrit3 ${ }^{-/}$retina (Fig. 5.4a) and confirmed that in the Lrit3 $^{-/}$mouse, TRPM1 expression was absent from the dendritic tips of the ON BCs (Fig. 5.4b). 
Antibodies are not available for nyctalopin, therefore I crossed and backcrossed a transgenic mouse line that expresses an EYFP tagged nyctalopin (TgEyfp-Nyc: Gregg et al., 2007) to Lrit3-^ mice. In TgEyfp-Nyc mice the EYFP-nyctalopin fusion protein is expressed in puncta in the OPL on the tips of the RBCs as well as large clusters representing the tips of the ON CBC terminals (Fig. 5.4a). Expression of the EYFP-nyctalopin fusion protein is absent in TgEyfp-Nyc:Lrit3-retinas. As expected, the absence of nyctalopin eliminated the expression of TRPM1 from the dendritic tips of both rod and cone Lrit3 ${ }^{-1-}$ ON BCs (Fig. 5.4b). Thus, LRIT3 expression is required for nyctalopin expression in both rod and cone $\mathrm{ON} \mathrm{BC}$ terminals and this result suggests that if there is a protein:protein interaction it should be between LRIT3 and nyctalopin. Whereas the absence of TRPM1 expression results from the loss of nyctalopin.

\subsection{LRIT3 is Expressed and Localized Normally in Other Models of cCSNB}

In the absence of LRIT3, the expression of many ON BC signalplex members remain (Lrit3nob6; Neuillé et a., 2015). In addition, in the $L$ rit3 ${ }^{\text {nob6 }}$ cone ON BCs, LRIT3 expression appears to be required for the expression and correct localization of mGluR6, GPR179, RGS7, RGS11 and Gß5 (Neuillé et al., 2015). Because the protein:protein interaction among members of this large signalplex is relatively unknown, we asked if LRIT3 was expressed in the absence of several ON BC signalplex proteins. Figure 5.5 shows that expression and localization of LRIT3 is independent of mGluR6 (Grm6-/, Maddox et al., 2008), GPR179 (Gpr179nob5, Ray et al., 2014), Nyctalopin (Nyx ${ }^{\text {nob }}$, Pardue, et al., 1998; 
Peachey et al., 2012; Pearring et al., 2011) or TRPM1 (Trpm1\%, Koike et al., 2010) at both the synapse with rod and cone terminals.
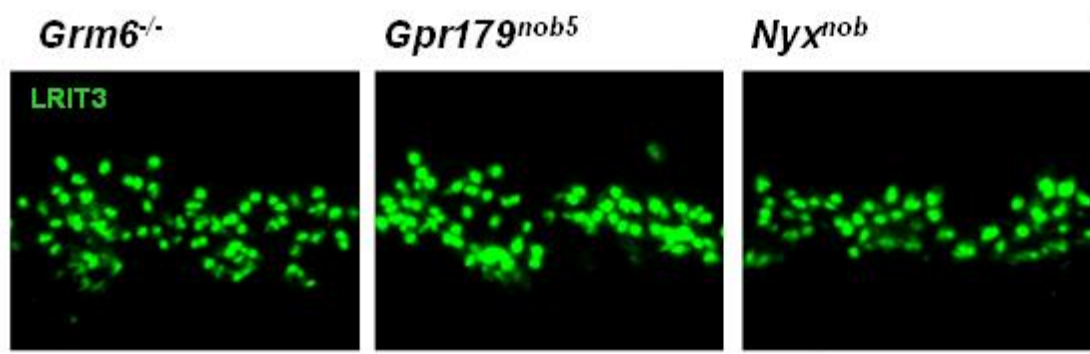

Trpm1\%
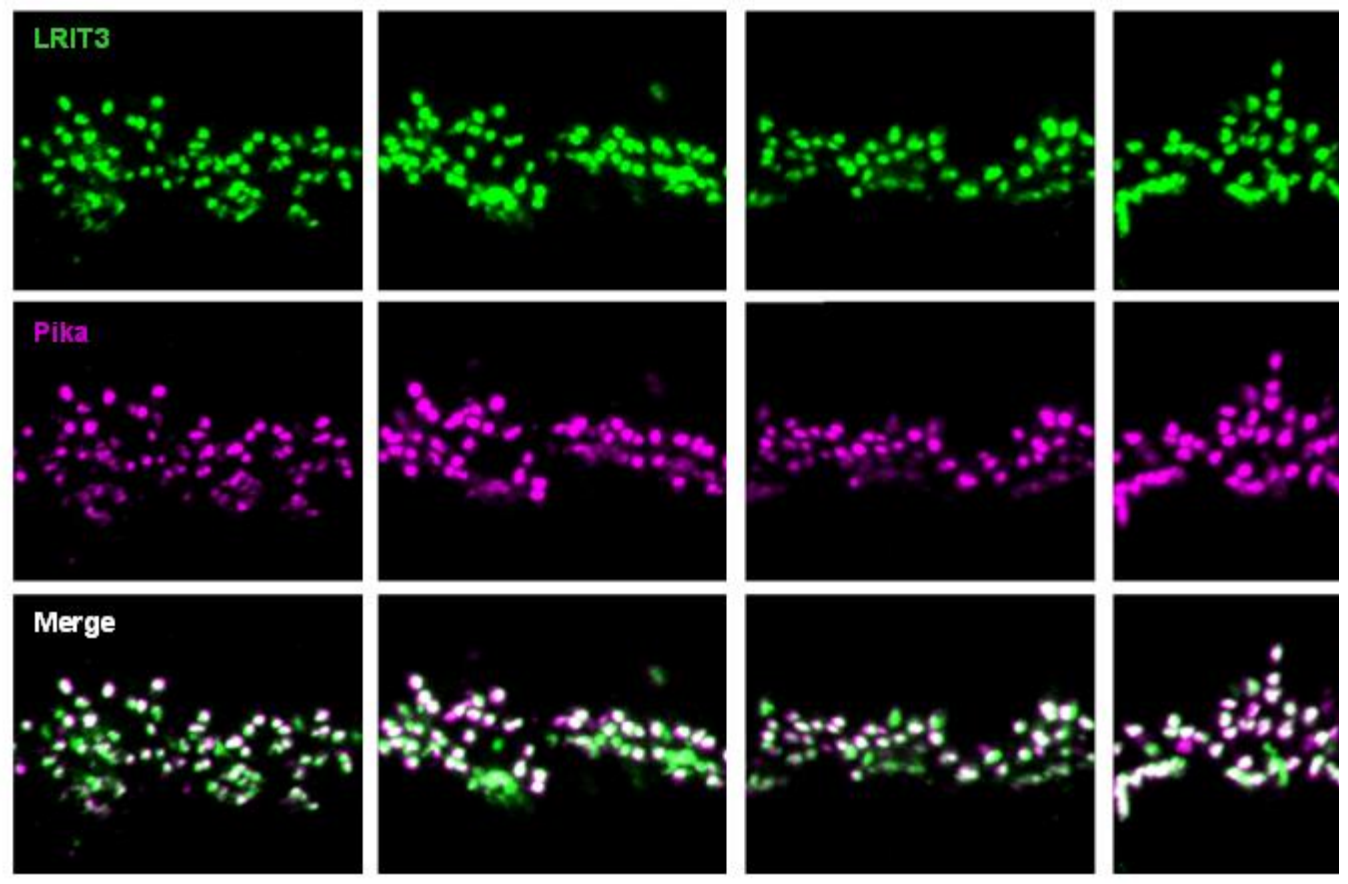

Figure 5.5. LRIT3 is expressed at both rod and cone photoreceptor terminals in several mouse models of cCSNB.

\subsection{LRIT3 is Required for Normal Function of Both ON and OFF GC Responses}

I surveyed general visual responses of Lrit3 $^{-\digamma}$ GCs to full field flashes by recording extracellular activity of a large number of GCs using the multielectrode array. Consistent with ERG responses Lrit3\% GCs in the dark adapted retina did not respond to either a $7 \times 10^{-4}$ or a $5 \times 10^{-3} \mu \mathrm{W} / \mathrm{cm}^{2}$ flash. The result was the same in $m$ GluR6 $6^{-}$GCs recorded under the same conditions. In contrast, 6 and $42 \%$ of 
WT GCs responded to the same flash intensities, respectively. To a photopic flash (46.3 $\left.\mu \mathrm{W} / \mathrm{cm}^{2} ; 500 \mathrm{msec}\right)$, nearly all WT and $m$ GluR6 ${ }^{-/}$GCs were visually responsive (Fig. 5.6a) but only about half of Lrit3- GCs had stimulus evoked responses. I classified the GCs with stimulus evoked responses based on the timing of their responses to light onset (ON) or light offset (OFF). Among WT GCs, the full-field photopic stimulus evoked responses at either light onset, offset or at both phases (Fig. 5.6a). These functional classes differed in both mGluR6 ${ }^{-/}$ and rrit3 $^{-/}$GCs, GCs with short latency ON responses were absent and all responses to light onset had long time to peak $>0.5 \mathrm{msec}$ after stimulus onset (Fig. 5.6aii). In contrast, OFF GCs across the three genotypes had similar time to peak ( $<0.3 \mathrm{msec})$. To determine whether all responses arise from input through OFF BCs, we recorded Lrit3 ${ }^{-}$GCs in control Ringer's and then in the presence of ACET. All light evoked responses $(n=15)$ were eliminated in the presence of ACET and all responses returned to control after drug washout. These results are consistent with both my ERG recordings and with a lack of glutamate release in the ON sublaminae.

The MEA recordings represent responses from a wide variety of GC classes. To better understand the parallel pathways involved, we assessed differences between Lrit3 $^{-/}$and control in three well characterized GCs; ON and OFF alpha, and OFF delta using patch clamp recordings in retinal whole mounts (Fig. 5.6b). The example traces (Fig. 5.6bi) show the representative averaged currents evoked at light onset and offset in three GCs of each type. At light offset, control ON alpha cells show a robust outward current that is somewhat sustained 


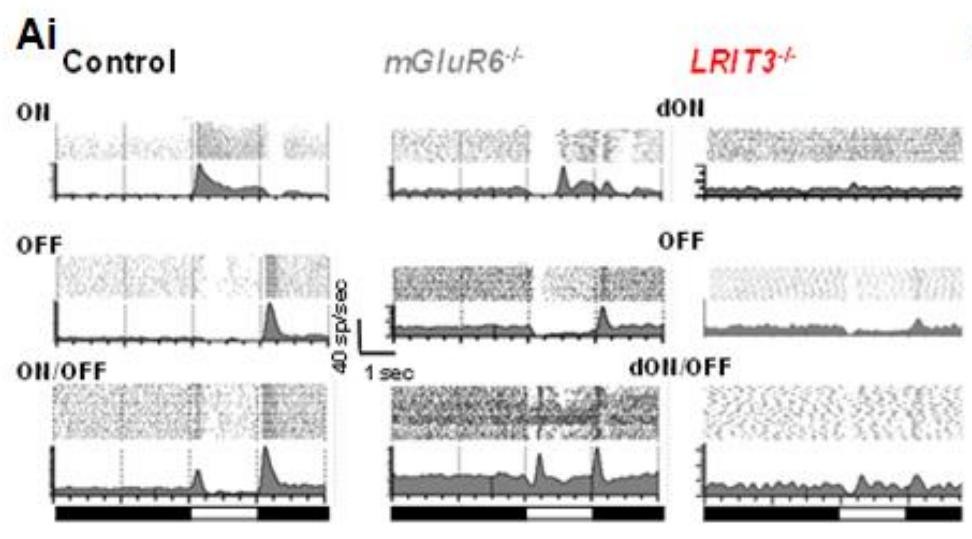

Aii

$\mathrm{Bi}$

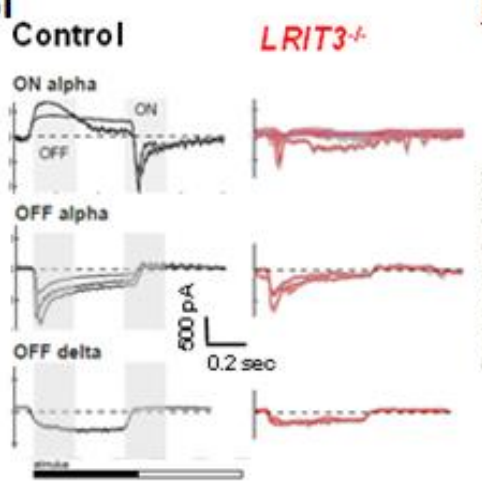

Bii

C
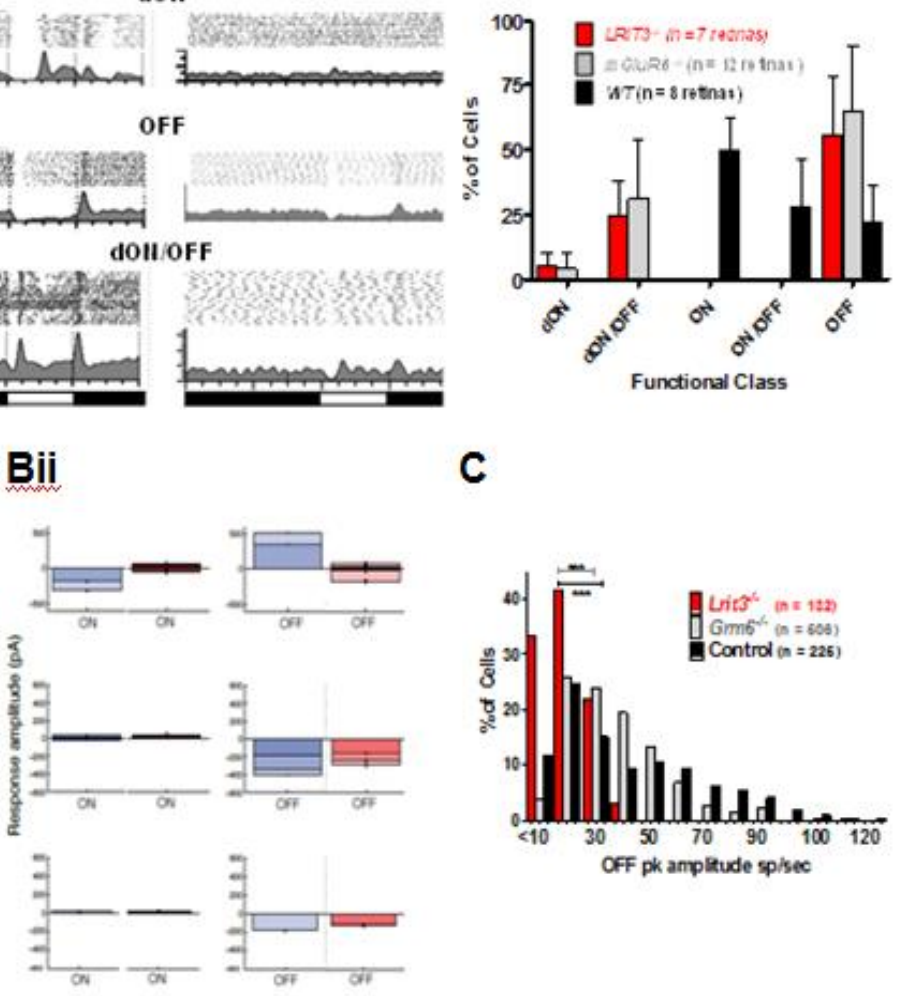

Figure 5.6. Visually-evoked responses of $\mathrm{Lrit3}^{-/-} \mathrm{GCs}$ are significantly altered compared to $\mathrm{Grm6}^{--}$and controls. Ai. Representative average peristimulus time histograms (above - raster plots to individual stimulus presentation) of responses recorded on a multielectrode array to a full field light stimulus $\left(46.3 \mu \mathrm{W} / \mathrm{cm}^{2}\right)$. Control GCs (comprised of $\mathrm{Lrit}^{+/}$, Grm6 $6^{+/}$and WT, which do not differ) responses can be classified as ON, OFF and ON/OFF from their excitatory response to light onset or offset, respectively. All responses to stimulus onset occur at $<0.4 \mathrm{sec}$ after stimulus onset. All Lrit3 ${ }^{-/}$and $\mathrm{Grm}^{-1}$ $\mathrm{GC}$ responses to light onset occur $>0.4 \mathrm{sec}$ after stimulus onset. These responses are referred to as dON. Aii. The distribution of GC functional classes is shown as a mean across animals $\left(\right.$ Lrit3 $^{+/} \mathrm{n}=7 ; \mathrm{Grm6}^{+/} \mathrm{n}=12$; and WT $\left.\mathrm{n}=8\right)$. B. Similar to the general population of GCs, the well characterized ON alpha GC has no excitatory response to light onset and the OFF alpha and OFF delta cells have significantly diminished peak ampitudes $(p<0.05)$. D. The Lrit $^{-}$GCs OFF responses have significantly small peak firing rates (10 sp/sec) compared to $\mathrm{Grm6}^{-/}$and control GCs (26 and $29 \mathrm{sp} / \mathrm{sec}$, respectively; Kruskal-Wallis; $\mathrm{p}<$ 0.001).

and light onset evokes a transient inward current. As expected Lrit3\% ON alpha GCs had no outward current at light onset, however, a small transient inward current remains and arises from crossover from the OFF pathway (Borghuis et 
al., 2014). At light offset, control OFF alpha GCs show a large initial transient inward current followed by a smaller sustained current that lasts for the duration of the flash and then returns to baseline. Control OFF delta GCs show a slowly building and sustained current to light offset that returns to baseline. In contrast, to $L$ rit3 $\%$ ON alpha GCs, the peak amplitude of the response to light offset was smaller in Lrit3\% OFF GCs compared to WT (Fig. 5.6bii). This result also was found in the spiking responses of all Lrit3\% OFF GCs compared to both WT and to $\mathrm{mGluR6} 6^{--}$GCs. As expected the data show the absence of signaling through the ON pathway. What is unexpected is the reduction in signaling through the OFF pathway, suggesting a trans-synaptic defect in the Lrit3\% retina that is not present in the $\mathrm{Grm6}^{-/}$retina. This defect could occur in OFF BC signaling or in synaptic transmission between the photoreceptor and the cone BC.

\section{Discussion}

Mutations in Lrit3 have been determined in two cCSNB patients to date (Zeitz et al., 2013). The Lrit3nob6 mouse has a targeted deletion of exons three and four, which should result in loss of the transmembrane and intracellular domains (Neuillé et al., 2014). The mouse we generated has a 40 bp deletion in exon two of Lrit3, the region coding for the leucine-rich repeat domain. Both mutations resulted in the absence of LRIT3 and characteristic nob phenotype (Fig. 5.1 \& Fig. 5.2). Therefore, deletions in the extracellular or transmembrane domains eliminate the protein. Both mouse lines also shared similar patterns of 
signalplex protein expression at the cone, but not rod synapse. This specific Lrit3\% phenotype is inherent to the absence of LRIT3 protein rather other genetic differences that might exist between the mice.

As previously reported, we observed mislocalization of TRPM1 to the soma in the absence of LRIT3. Similar to TrpM1\%, application of the TRPM1 agonist, capsaicin, produced a negligible current response (Fig. 5.2), consistent with the absence of TRPM1 protein in the synapse. The Lrit3 ${ }^{--}$alterations in TRPM1 expression and disruption in TRPM1 function resembles the Nyx ${ }^{\text {nob }}$ phenotype (Gregg et al., 2007; Pearring et al., 2011). Because of this, we investigated the expression of EYFP tagged nyctalopin in Lrit3--/TgEyfp-Nyc retinas.

The nyctalopin-EYFP fusion protein expressed in TgEyfp-Nyc mice is functional and capable of restoring ON responses in Nyx $x^{\text {nob }} / T g E y f p-N y c$ mice (Gregg et al., 2007). The absence of EYFP tagged nyctalopin in Lrit3--/TgEyfpNyc is consistent with the idea that nyctalopin expression is dependent on LRIT3 expression. This is indicative of a protein-protein interaction between LRIT3 and nyctalopin. LRIT3 expression in Nyx ${ }^{\text {nob }}$ and LRIT3 and nyctalopin expression in TrpM1\% retinas suggest a one direction path of dependence (Fig. 5.4; Pearring et al., 2011). Nyctalopin is required for TRPM1 expression and LRIT3 is required for nyctalopin expression. Therefore, a protein-protein interaction may be inferred between nyctalopin and LRIT3, but not TRPM1 and LRIT3. Pearring and colleagues (2011) confirmed a direct interaction between TRPM1 and a small fraction of the nyctalopin protein and nyctalopin is required for the proper 
localization of TRPM1 in ON BC dendritic tips. Mislocalization of TRPM1 in Lrit3\% is likely due to the loss of the protein-protein interaction between nyctalopin and TRPM1 rather than LRIT3 and TRPM1. Further studies are necessary to determine this. Nyctalopin lacks an intracellular domain and it has been assumed there is another ancillary transmembrane protein linking extracellular nyctalopin to TRPM1 and intracellular scaffolding proteins at the synapse (Pearring et al., 2011). Determining the whether LRIT3 interacts directly with TRPM1 and is the linking protein will clarify this question.

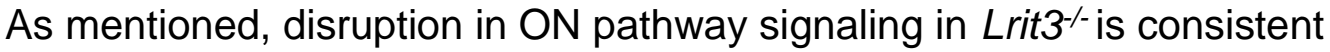
with other mouse models of cCSNB. Surprisingly, however, signaling through the OFF pathway was also significantly reduced in the absence of LRIT3. OFF BCs express Kainate receptors (Borghuis et al., 2014) which are not affected by loss of ON BC signal cascade proteins. Further, GC spiking response to light decrements are similar between $\mathrm{Grm}^{+/+}$and $\mathrm{Grm6}^{-/-}$mice (Fig. 5.6; Renteria et al., 2006). Kainate puffs evoked similar inward currents in Lrit3 ${ }^{-1+}$ and Lrit3-- OFF $\mathrm{BCs}$, confirming the presence of functional glutamate receptors in the dendrites of these cells (Fig. 5.3). However, EPSCs and membrane depolarizations to a luminance decrement are significantly lower in Lrit3\% OFF BCs compared to Lrit3 $^{-/+}$(data not shown), suggesting decreased glutamate transmission from the cones to the OFF BCs. Lower excitatory currents and spiking activity in Lrit3\%GCs compared to WT GCs correlated with the OFF BC current responses. Only 19\% of Lrit3 $^{-/}$GCs compared to $91 \%$ Lrit3 $^{-/}$and $73 \%$ Grm6 $^{-/}$had light responses. Also, Lrit3- OFF GC spiking response amplitudes were lower than 
Lrit3 $^{-/ 4}$ and $\mathrm{Grm6}^{-/}$. Loss of Grm6 $6^{-/}$and Lrit3 ${ }^{-/}$responses in the presence of the Kainate receptor antagonist, ACET confirmed their origin in the OFF pathway. The decreased excitatory currents in the OFF pathway leads us to conclude that the origin of the OFF pathway signaling defect is decreased glutamate transmission from the cone to OFF BC. Impaired signaling transmission in the absence of LRIT3 could be the result of decreased glutamate release from the cones or structural abnormalities in the synapse.

ON BC cascade proteins are downregulated in cone, but not rod BCs. This cone specific phenotype suggests that while LRIT3 is expressed at both the rod and cone synapse, its function is different at the two locations. LRIT3 is required for nyctalopin and TRPM1expression at both the rod and cone synapse, but LRIT3 is also required for expression of many other proteins as well at the cone synapse. One of these proteins is the PNA binding protein that is specific to the cone and not rod synapse. In light of this, the identification of the PNA binding protein at the base of the cone terminals is of significant interest given its prospective role in regulating structure or function at the cone synapse. LRIT3 could be anchoring specific signalplex at the cone, but not the rod terminals directly. But most likely, LRIT3 may regulate the expression or function of another protein that is required for cone BC signalplex localization. Overall, LRIT3 may have a trans-synaptic function which maintains nyctalopin in the rod and cone ON BCs, in required for visual signal transmission through the ON BCs, and enhances signaling from the cones to OFF cone BCs. 


\section{CHAPTER VI \\ GENERAL DISCUSSION AND FUTURE DIRECTIONS}

TRP channels participate in multiple functions in neurons, with the majority of them playing critical roles in sensory processing (Venkatachalm \& Montell, 2007). TRPV1 is the most characterized TRP channel outside of the retina (Caterina et al., 1997; Caterina \& Julius, 2001) and its roles in thermosensation and pain signaling have been well defined. While TRPV1 is present in brain nuclei (Cavanaugh et al., 2011b), the biological function of TRPV1 in signal transduction in the CNS is inconclusive (see Ho et al., 2012 for a review). Very little is known about TRPV1 in the retina. TRPV1 function may be neuroprotective or neurodegenerative in response to retinal ischemia (Sakamoto et al., 2014), and glaucoma (Ward et al., 2014; Weitlauf et al., 2014; Sappington et al., 2015). The function of TRPV1 in shaping visual signals in the retina has not been investigated.

With very little prior knowledge regarding the role of TRPV1 in normal visual processing, I investigated this by comparing the GC spiking responses in WT and $\operatorname{TrpV} 1 \%$ mice, which represents the visual output of the retina. I used knowledge of the retinal pathways and circuitry to determine whether TRPV1 modulates normal visual signals and if so, which pathways TRPV1 shapes. I 
simultaneously determined the specific cells in which TRPV1 is expressed and used knowledge of the known function of those cells to piece together the role of TRPV1 in the retina. My cohesive results from Chapters III and IV determined TRPV1 is regulating a circuit that is likely involved in enhancing visual acuity in daylight and visual sensitivity in dim light. TRPV1 enhances serial inhibition of GABAergic $A C$ s to reduce $G A B A$ release onto $G A B A_{c}$ receptors, which are located on ON CBCs. With decreased GABAergic inhibition of the ON CBCs, glutamate release is prolonged, resulting in sustained spiking responses and enhanced spontaneous activity in post-synaptic ON GCs. Sustained ON GCs improve the perception of static details in the visual world (Roska et al., 2006). TRPV1 in glycinergic ACs (Chapter IV) likely enhances their tonic release of glycine onto GABAergic amacrine cells to mediate the serial inhibition.

\section{Discussion of Experimental Limitations}

My results provide a broad idea of TRPV1 function in retinal circuitry. From here, the details of the circuits can be dissected to test my conclusions and understand more about this protein and the neural pathways it affects. There are inherent limitations to conclusions regarding underlying retinal circuitry based on the spiking output alone. Making evidence based assumptions about current inputs and outputs without direct measurement is a limitation of interpreting spiking activity. One assumption I made regarding the role of TRPV1 in retinal processing was that it modulates signals presynaptic to the GCs. The evidence for this includes the following: 
1. TRPV1 is expressed in small populations of GCs which total only $\sim 6 \%$ of all GCs (Chapter IV). In contrast, TRPV1 affects either spontaneous and/or light evoked spiking activity in the majority ( $>70 \%)$ of ON and OFF GCs (Chapter III). In contrast, TRPV1 is expressed in large numbers of ACs whose processes form a continuous plexus in the IPL. This strongly suggests TRPV1activity in ACs is affecting the spiking activity in the majority of GCs through presynaptic modulation of the visual signal. However, based on this finding, I cannot determine whether TRPV1 in the small percentage of GCs is affecting their spiking activity. Therefore, I tested idea this directly.

2. TRPV1 is a calcium channel which can be activated by capsaicin. With prolonged exposure to capsaicin, TRPV1 activation results in prolonged $\mathrm{Ca}^{2+}$ influx lasting approximately three min. (DRG, Medvedeva et al., 2009; GCs, Ryskamp et al., 2015) followed by a decline in intracellular $\left[\mathrm{Ca}^{2+}\right]$ to a plateau which is elevated compared to pre-stimulus. The plateau lasts tens of minutes depending on the strength of stimulus (i.e. concentration of capsaicin) and represents TRPV1 mediated release of $\mathrm{Ca}^{2+}$ from intracellular stores (Medvedeva et al., 2009). TRPV1 is expressed in two populations of ipGCs, M2 and M1, and an OFF GC, B3o. Using whole cell patch clamp, I recorded from each of the TRPV1+ GC types. In voltage clamp, I held the GC at the chloride reversal potential ($60 \mathrm{mV}$ ) in the presence of $30 \mu \mathrm{M}$ capsaicin. No inward current greater than control was evoked by capsaicin in any TRPV1+ GCs. In contrast, inward 
current responses to capsaicin were detected in all three tested TRPV1+ ACs. From this I concluded that TRPV1activation in ACs produces $\mathrm{Ca}^{2+}$ influx. However, the absence of either a capsaicin evoked intial rise in inward current or a slow plateau phase in TRPV1+ GCs suggests TRPV1 is not present in the retinal portion of GCs or is not activated by capsaicin. I therefore have no evidence that TRPV1 in GCs is modulating spiking activity directly in the normal wholemount retina.

One limitation in my data is the lack of direct current recordings in $\mathrm{ON}$ GCs that are affected by TRPV1 activity. My attempts to identify the specific GC classes have been unsuccessful. If the specific classes are identified, I could record spontaneous and light evoked currents in patch clamp configuration to determine the source of the altered spiking activity. In Chapter IV, I determined that TRPV1 most likely regulated feedback or feedforward inhibition to sON GCs. I attempted to identify a specific morphological class of ON GC whose sustained light responses became transient in the absence of TRPV1. To do this, I crossed and back crossed TrpV1-/ mice with GRIK, SLICK-H, and PVcre/STP reporter mouse lines with fluorescently labeled population of sON GCs. I obtained homozygous TrpV1-//heterozygous labeled line mice (Table 6.1). I targeted fluorescently labeled ON GCs in retinal wholemount preparation (note quadrants of the retina were used) using brief illumination. I recorded the extracellular lightevoked spiking activity to full-field $(5 \mathrm{sec})$ light stimulation. I found that the sON GCs in these labeled lines maintained their sustained spiking responses in the 
absence of TRPV1. I concluded that TRPV1 did not modulate the temporal spiking activity of those specific GCs, which resemble the A1 and A2 (Sun et al., 2002).

Other mice with labeled sON GCs have recently been developed and I propose continuing these experiments using the labeled lines described in table 6.2. I will record spiking responses to full-field light in cell attached mode. If there is a response to a luminance step that is transient in the $\operatorname{TrpV1} 1^{-1}$, I will break in

Table 6.1 Mouse lines with fluorescently labeled GCs, which I crossed with $\operatorname{TrpV1}{ }^{-/}$. I targeted labeled sON GCs and recorded spontaneous and light evoked responses in whole cell patch clamp mode. TRPV1 did not alter the temporal properties of sON GCs that are labeled in these mice.

\begin{tabular}{|c|c|c|c|c|}
\hline Mouse & $\begin{array}{l}\text { Short } \\
\text { Name }\end{array}$ & $\begin{array}{l}\text { GC } \\
\text { labeled }\end{array}$ & $\begin{array}{l}\text { Spiking } \\
\text { Responses } \\
\text { in TrpV1 } 1^{-/}\end{array}$ & $\begin{array}{l}\text { Source/Jackson } \\
\text { lab stock\# }\end{array}$ \\
\hline $\begin{array}{l}\text { C57BL/6-Tg(Grik4- } \\
\text { cre)G32-4St//J }\end{array}$ & GRIK4 cre & ON A1 & Sustained & $\begin{array}{l}\text { Gift from S. } \\
\text { Bloomfield \& A. } \\
\text { Huberman; 006474 }\end{array}$ \\
\hline $\begin{array}{l}\text { Tg(Thy1-cre/ERT2,- } \\
\text { EYFP)HGfng/PyngJ }\end{array}$ & SLICK-H & ON A1 & Sustained & $\begin{array}{l}\text { Gift from S. } \\
\text { Bloomfield \& A. } \\
\text { Huberman; } 012708\end{array}$ \\
\hline $\begin{array}{l}\text { B6;129P2- } \\
\text { Pvalbtm1/(cre)Arbr/J }\end{array}$ & PV're & & & $\begin{array}{l}\text { Hippenmeyer et al., } \\
\text { 2005; } 008069\end{array}$ \\
\hline Thy1 ${ }^{\text {Stp-EYFP }}$ & STP & & & $\begin{array}{l}\text { Gift of J. Sanes; } \\
\text { Buffelli et al., 2003; } \\
005630\end{array}$ \\
\hline & 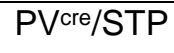 & ON, A2 & Sustained & \\
\hline
\end{tabular}

and record light responses to full-field and spot stimuli using whole cell mode to will isolate and record either the IPSCs $\left(V_{\text {hold }}=\sim 0 \mathrm{mV}\right)$ or EPSCs $\left(V_{\text {hold }}=\sim-60\right.$ $\mathrm{mV}$ ). Spontaneous currents (sEPSCs and sIPSCs) (absence of light stimulation) and light evoked currents (L-EPSCs and L-IPSCs) will be characterized to stimuli similar to those used in the intact preparation (Chapter III). 
Table 6.2 Mouse lines with fluorescently labeled GCs, which can be crossed with $\operatorname{TrpV1}$ - . Labeled sON GCs can then be targeted for patch clamp recording experiments to determine the role of TRPV1 in shaping light responses

\begin{tabular}{|c|c|c|c|}
\hline Mouse & Short Name & $\begin{array}{l}\text { GC } \\
\text { labeled }\end{array}$ & Reference \\
\hline Opn4CreERT2 & OPN4 ${ }^{\text {cre }}$ & & Chen et al., 2011 \\
\hline $\begin{array}{l}\text { B6.129(Cg)-Tg(CAG- } \\
\text { Bgeo/GFP)21Lbe/J }\end{array}$ & ZEG & & \\
\hline & OPN4cre/ZEG & M1, M2, M3 & Chen et al., 2011 \\
\hline $\begin{array}{l}\text { Tg(Cdh3- } \\
\text { EGFP)BK102Gsat/Mmnc }\end{array}$ & CDH3-GFP & $\begin{array}{l}\text { Multiple ON } \\
\text { types }\end{array}$ & Osterhout et al., 2011 \\
\hline $\begin{array}{l}\text { Tg(Hoxd10- } \\
\text { EGFP)LT174Gsat/Mmucd }\end{array}$ & Hoxd10-GFP & $\begin{array}{l}\text { ON DS and } \\
\text { ON/OFF DS }\end{array}$ & Dhande et al., 2013 \\
\hline
\end{tabular}

In the absence of TRPV1, I expect to find light evoked EPSCs with shortened

duration compared to WT. I also predict the $\mathrm{GABA}_{c}$ antagonist TPMPA will prolong the EPSCs and subsequent spiking activity. Light evoked IPSCs may also have prolonged duration compared to WT. If the temporal properties of these labeled GCs is similar in WT and $\operatorname{TrpV1}^{-1}$, alternative approaches include the following:

1. Similar experiments to MEA recordings conducted in Chapter IV can be used to assess the light responses with and without the GABAc receptor blocker, TPMPA. I expect response properties, including durations to increase in WT, but proportionally more in $\operatorname{TrpV} 1 \%$. However, there are limitations to this experiment, which are discussed in appendix I.

2. Mouse lines with labeled ON BCs can be crossed to TrpV1\%. Using two-photon microscopy, BCs can be targeted for patch clamp recording in wholemount retinas and light evoked current responses can be recorded. I expect the excitatory input to labeled BCs to be similar in the absence of TRPV1. TPMPA sensitive inhibitory input may be increased.

3. Since the majority of ON GCs had lower spontaneous activity in the absence of TRPV1, spontaneous currents can be recorded from labeled 
ON GCs. Pharmacological manipulation can be used to isolate the source of the currents.

My data in Chapters III and IV show TRPV1 expression within a small percent of GCs that is not activated by capsaicin. This is contradictory to other reports. Using TRPV1 antibodies, Sappington and colleagues (2011\& 2015), report robust expression of TRPV1 in the majority of GCs. Using the same antibody and concentrations they described, I found similar fluorescent expression in the mouse retina, however I also found the same expression in $\operatorname{TrpV1}{ }^{-/}$retina. Further, the fluorescent expression in the DRG of WT and TrpV11- mice was similar and did not represent the DRG expression of TRPV1 reported by multiple investigators (Hwang et al., 2005; Zacharova \& Palecek, 2009; Cavanaugh et al., 2011b). The same group, in a series of publications, used calcium imaging in dissociated GCs to measure increases in intracellular $\left[\mathrm{Ca}^{2+}\right]$ in response to capsaicin. They report that TRPV1 is present in the majority of GCs and is activated by capsaicin, which leads to an influx of $\mathrm{Ca}^{2+}$. While that group did not repeat the experiment in $\operatorname{TrpV} 1^{-1}$, Ryskamp and colleagues (2014) report similar capsaicin mediate $\mathrm{Ca}^{2+}$ signals in dissociated GCs, which are absent in the $\operatorname{TrpV} 1 \%$.

The most likely explanation for the difference between my results and the two other groups are the different experimental models. I recorded current responses to capsaicin from tdTomato+ GCs in TRPV1 reporter wholemount retinas. The other groups looked at calcium signals in response to capsaicin in 
dissociated GCs. The following are possible explanations for the differences in the calcium signals and my absence of current responses.

1. TRPV1 expression in the membrane is increased in dissociated GCs compared to wholemount. Investigation of TRPV1 in the PNS has confirmed that TRPV1 expression is regulated by external factors. When the cell is stressed (as would occur in the process of retinal cell dissociation) TRPV1 protein in the membrane increases rapidly. In primary afferent nociceptors, TRPV1 is present in intracellular vesicles. In the presence of stressful stimuli, the nociceptors are depolarized and the vesicular TRPV1 protein is rapidly inserted in the membrane (PlanellsCases et al., 2005). At the same time, TRPV1 transcription is upregulated. There is evidence that GCs contain similar machinery and upregulate TRPV1 in response to increased pressure or ischemia (Ward et al., 2014; Sappington et al., 2015; Sakamoto et al., 2014).

2. Capsaicin may be activating TRPV1 located in intracellular compartments which may release calcium from intracellular stores. This would produce an increased calcium signal but not necessarily a change in the membrane voltage.

3. GCs which express TRPV1 are more likely to survive the dissociation process.

To determine the source of the differences between the reported calcium signals and my current responses to capsaicin application, I propose doing calcium imaging in wholemount retina. To first, confirm capsaicin responses in 
ACs but not GCs in larger populations of TRPV1 reporter cells, I propose similar experiments to those conducted in Chapter III with calcium imaging in TRPV $1^{\text {cre }}$ mice crossed to Gt(ROSA)26Sortm38(CAG-GCaMP3)Hze (Ai38) mouse. The calcium indicator protein, GCaMP3, will be expressed in TRPV1+ cells and TRPV1 agonist mediated calcium responses can be imaged simultaneously in larger populations. Current responses in capsaicin activated neurons can be confirmed by patch clamp recordings in synaptically isolated cells. Based on my data in Chapter IV in which I recorded capsaicin induced currents in tdTomato+ ACs but not GCs, I expect capsaicin activated $\mathrm{Ca}^{2+}$ signals and currents in ACs, not GCs in wholemount TRPV1 ${ }^{\text {cre }} / \mathrm{Ai} 38$ retina. Depending on the results of these experiments, further experiments can be designed to investigate whether TRPV1 is upregulated in response to trauma or if capsaicin evokes release of $\mathrm{Ca}^{2+}$ from intracellular stores.

To investigate the third possibility, that TRPV1+ GCs are more likely to survive the dissociation procedure, I propose dissociating TRPV1 1 cre/Ai9 retinas according to published methods (Ryskamp et al., 2014) and counting the numbers of fluorescent and non-fluorescent GCs in several samples. I would place a sample of the solution containing the dissociated retina on a slide, also using a similar technique to those published, and fix the cells using $4 \%$ paraformaldehyde. I would use standard protocols to label the samples using antibodies to tdTomato and a GC marker such as the transcription factor, recombination signal-binding protein Jk (RBP-J; Zheng et al., 2009). I would count the number of tdTomato+/RBP-J+ cells in each sample and compare it to 
the number of RBP-J+ only cells. Given my data from Chapter IV, I expect $6 \%$ of GCs to be tdTomato+. With this expectation I would collect samples from 3 to 6 mice to obtain appropriate power ( $G^{*}$ Power).

Another limitation in the interpretation of my results from Chapter III is the unknown mechanism by which TRPV1 is activated. Recent evidence suggests TRPV1 in intracellular locations is activated by endovanilloids and regulates release of endocannabinoids there in a pathway which regulates tonic GABA release in hippocampal neurons (Lee et al., 2015). Enzymes responsible for synthesis endocannabinoids and endovanilloids such as anandamide, HpETE and NADA are expressed in mouse ACs (Porcella et al., 2000; Hu et al., 2010). Endovanilloids within or post-synaptic to the TRPV1+ neurons have the potential to activate TRPV1 (Gibson et al., 2008; Ho et al., 2012). If they are activating TRPV1, their expression must be colocalized with or adjacent to TRPV1 receptors. I propose labeling TRPV1 ${ }^{\text {cre }} /$ Ai9 retinal sections with antibodies to various proteins related to endovanilloid expression and assessing the localization of endovanilloids related to tdTomato+ neurons. If endovanilloids are found in or adjacent to tdTomato+ neurons, further experiments can be designed to test whether the endovanilloids are activating TRPV1 and affecting visual responses.

The final limitation of my data I will discuss is the assumption that TRPV1 is directly regulating the release of the traditional neurotransmitters, glycine and GABA. Other neurotransmitters are expressed by retinal cells and release of such non-traditional neurotransmitters can be regulated by TRPV1 (Leonelli et 
al., 2009; Castillo, 2012; Leonelli et al., 2015). One such neurotransmitter that might affect visual responses is nitric oxide (NO). I determined TRPV1 had a specific effect on which were indicative of TRPV1 enhancing glycine release from glycinergic $A C s$, resulting in disinhibition of GABA release to $G A B A_{c}$ receptors. Consistent with that idea and previous publications (Leonelli et al, 2011 \& 2013), my results in Chapter IV confirm the expression of TRPV1 in the inner retina of mice. The TRPV1+ Flag A AC expresses glycine, while the TRPV1+ nGnG AC has no known neurotransmitter. Also, a GABAergic WF AC resembles the recently describe nNOS-2 (Zhu et al., 2014), and therefore has the potential to release a second neurotransmitter, nitric oxide (NO).

The similarities between the function of NO and TRPV1 in the retina is evidence that TRPV1 has the potential to regulate NO, as described below:

1. The function of NO in the retina: NO modulates visual signals in similar ways as TRPV1. NO enhances the coupling between the All and ON CBC terminals (Bloomfield et al.,1997; Bloomfield and Völgyi, 2009). $\mathrm{NO}$ also regulates $\mathrm{GABA}$ function in rod $\mathrm{BCs}$ through intracellular pathways (Gasulla et al., 2012). Both of these processes affects visual signal transmission through the primary rod pathway. In this way, NO enhances the sensitivity of ON and OFF GC responses to dim light. Similarly, my results show that TRPV1 activity is required for signaling through the primary rod pathway and enhances the sensitivity of ON and OFF GCs to dim light. 
2. Further, NO-stimulated cGMP activation enhances the sustained release of glutamate from cone BCs directly in light adapted conditions (Snellman et al., 2009; Vielma et al., 2014), and NO can modulate the release of glycine and GABA from ACs directly and inhibit GABA receptor function (Wexler et al., 1998). Similarly, my results show that TRPV1 enhances the sustained release of glutamate from cone BCs in light adapted conditions. And the modulation of light responses by TRPV1 are consistent with TRPV1 enhancing the release of glycine from ACs or decreasing GABAergic inhibition.

While my results described in Chapter III are consistent with a role for TRPV1 in sustained glycine release from the Flag A ACs, NO (and a possible neurotransmitter in the nGnG ACs) also has the potential to alter the visual responses. Based on this, it is important to: 1 . Determine if TRPV1+ ACs express nNOS and release NO. 2. Determine if TRPV1 regulates the release of NO. I propose the following hypotheses:

1. $\mathrm{nNOS}$ is expressed in TRPV $1+$ ACs

2. TRPV1 is required for $\mathrm{NO}$ release from nNOS+ACs.

I propose the following experiments to test my hypotheses:

1. To investigate the prospective co-expression of $\mathrm{nNOS}$ and TRPV1, I will label the TRPV1 ${ }^{\text {cre}} /$ ai9 retinas with antibodies to nNOS. If my hypothesis is 
correct, the tdTomato and nNOS antibody expression should colocalize in ACs.

2. To determine if TRPV1 regulates NO release I propose a series of experiment.

A. nNOS protein expression was downregulated by intravitreal capsaicin injections (Leonelli et al., 2013), which may suggest TRPV1 or TRPM1 activity downregulates nNOS expression in the retina. To test if TRPV1 modulates nNOS protein levels, I propose comparing nNOS protein expression in $\operatorname{TrpV}^{-1}$ and WT retinas by assessing the nNOS antibody label and western blot quantification in each genotype. Since nNOS is also modulated by light and circadian rhythms, assessments should be made during the circadian day and night as well as in both dark and light adapted retinas. If my hypothesis is correct that TRPV1 enhances NO release, then I expect nNOS protein expression will be decreased in TrpV1\% compared to WT.

B. NO release in the light and during the circadian day reduces gap junction coupling between the All and ON CBC in order to enhance cone dominated vision (Zhang et al., 2005; Volgyi, \& Bloomfield, 2011). Neurobiotin passes through gap junction and is widely used to assess coupling between cells. To determine if TRPV1 enhances NO release, I propose assessing the All to ON CBC gap junction coupling in TrpV1\% and WT retinas. To do this, neurobiotin will be injected in All ACs and/or ON CBCs in WT and TrpV1\% retinas. Since gap junction coupling is 
modulated by light and circadian rhythms, assessments should be made during the circadian day and night as well as in several luminance conditions. If my hypothesis is correct, the transfer of neurobiotin between All and ON CBCs will be increased in TrpV1\% compared to WT during the circadian day and in daylight conditions.

C. Under light adapted conditions increased NO modulates the release of glycine and GABA from ACs and inhibits GABA receptor function in ACs (Wexler et al., 1998). ON CBCs express GABA $A$ and $G A B A_{c}$ receptors and $N O$ enhances release of glutamate from ON CBCs. To test if TRPV1 modulates the same pathways through release of NO or glycine, I propose recording spontaneous and evoked GABAergic inhibitory currents in WT and TrpV11- ON CBCs in light adapted conditions. To target specific BC types, mice with fluorescently labeled ON BCs can be crossed and backcrossed (VSX type 7, Gus-GFP type 7, clm1 type 9, GRM6-tdTomato all ON) to $\operatorname{TrpV} 1 \%$. If there is a difference in the charge transfer between genotypes, then $\mathrm{GABA}_{A}$ and $\mathrm{GABA}_{c}$ selective inhibitors can be applied to determine the source of the difference. Given my GC spiking results in Chapter III, I expect increased $\mathrm{GABA}_{c}$ mediated inhibition in type $6,7,8$ or $9 \operatorname{TrpV1} 1-\mathrm{ON}$ BC compared to WT. nNOS inhibitor (ARL-17477 dihydrochloride hydrate) also can be applied. If my hypothesis is correct, nNOS inhibitor will enhance GABA $c$ mediated current in WT ON BCs and have no effect on TrpV1\% ON BC inhibitory current. Glycine receptor antagonist, strychnine, may also be 
applied. If TRPV1 enhances glycinergic serial inhibition of GABAergic ACs, the GABAergic inhibition should be increased in WT ON BCs in the presence of strychnine, but not in TrpV1/ ON BC.

If the results of these experiment is consistent with TRPV1 regulating NO release, then further experiments can be designed to test the hypothesis directly, such as NO imaging using the DAF-FM NO fluorescent indicator or recording GABAergic inhibitory currents in ON CBCs with NOS inhibitors.

\section{Future Experiments}

To assess the specific functions of TRPV1+ GCs, it helps to know the specific brain nuclei the GCs project to. The axonal projections from TRPV1+ GCs provide very little contribution to the dLGN, the image forming area (Chapter IV). Also, my data suggests that the TRPV1+M1 ipGCs project to the SCN but not the OPN and likely mediate specific functions there. One of the main functions of GCs projecting to the SCN is circadian photoentrainment. I propose determining whether TRPV1 plays a role in circadian photoentrainment by comparing the activity of TrpV1- mice compared to WT. Circadian photoentrainment is typically assessed by tracking the activity of mice during 12hr light and 12-hr dark days using a running wheel (Altimus et al., 2010). Mice are more active during the dark phase. The light-dark phase can then be advanced 6 hrs and the activity of WT mice will adjust so that they are more 
active 6 hrs earlier, or will photoentrain. Mice which lack photoreception through SCN projecting ipGCs will not photoentrain to light shifts and increase activity according to their circadian rhythms only, known as free running (Provencio \& Foster, 1995; Panda et al., 2002). I hypothesize that TrpV1\% mice will still show photoentrainment, however the ability of the mice to adjust their running patterns to the light shift will be decreased compared to WT.

To determine other possible contributions of TRPV1 to light pathways in the brain, I propose experiments to determine the specific brain nuclei innervated by each class of TRPV1+ GC. To do this I can inject a retrograde neuronal tracer such as a dye conjugated Cholera Toxin B into the SCN, OPN, LGN, IGL, or SC

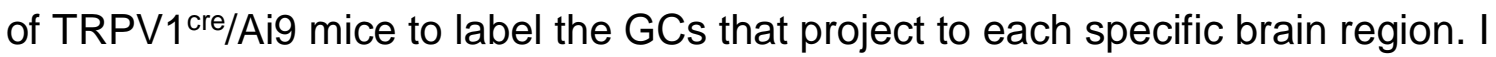
can then analyze the morphologies of the tdTomato+/CT-B+ neurons to determine the specific GC classes that project to each region. I hypothesize that tdTomato+ M2 GCs project to the IGL and OPN, M1 GCs project to the SCN, and B3o GCs project to the IGL and SC. Depending on the results of these experiments, other techniques can be employed to address the function of TRPV1 in GCs.

\section{TRPM1}

In contrast to TRPV1, TRPM1 is the most characterized TRP channel in the retina due to its role in signal transduction through the ON pathway. Loss of TRPM1 signaling causes the visual disorder, cCSNB. However, very little is 
known about its function outside of the retina. TRPM1 also is expressed in melanocytes and may regulate intracellular $\mathrm{Ca}^{2+}$ concentrations and melanogenesis (Guo et al., 2012).

\section{Discussion of Experimental Limitations}

Our data shows that LRIT3 is required for nyctalopin expression rod and cone BC dendrites. In the absence of LRIT3 and subsequent nyctalopin, TRPM1 is mislocalized and ON pathway signaling is disrupted, resulting in the cCSNB phenotype in patients. We further showed that LRIT3 is required for normal glutamate transmission from the cone terminals to cone BCs. In the absence of LRIT3, responses through the OFF pathway are lower than WT. It is unknown what additional effect the decreased OFF signaling might have on the vision of patients with Lrit3 mutations. The two patients had clinical symptoms similar to patients with Nyx mutations (Zeitz et al., 2013). Besides the nob ERG and night blindness, the first patient had high myopia, strabismus, and decreased visual acuities in both eyes (20/80 and 20/30 corrected). The second patient also had myopia and visual acuities of $20 / 40$ and $20 / 50$ corrected. Both patients also reported blurry vision. Patients with Nyx mutations also often have myopia, reduced visual acuity, and strabismus.

It is unknown if OFF pathway signaling is decreased in humans with Lrit3 mutations and if so, whether it alters visual perception more than mutations in Nyx. Tests such as the pattern ERG and visually evoked potential which measure inner retinal and $\mathrm{V} 1$ function, respectively, might be useful measures in 
these patients. Visually evoked potentials from patients with Nyx mutations are absent to dim luminance, but similar to normal vision subjects under photopic conditions (Kubová et al., 2004). Patients with LRIT3 mutations might be expected to have visually evoked potentials with decreased amplitudes.

\section{Future Experiments}

We determined a transynaptic functional role for LRIT3 in glutamate transmission at the cone synapse. Current and future experiments are designed to investigate the mechanisms by which LRIT3 enacts its effect. Specific questions and prospective experiments to address them are listed below.

1. Does LRIT3 bind nyctalopin and/or TRPM1 directly?

Protein interact can be assessed using pull-down studies with LRIT3 and TRPM1 antibodies with retinal lysates. Tg-Nyx mice can be used to assess interactions with nyctalopin in the absence of a nyctalopin antibody.

2. What other protein(s) does LRIT3 interact with that relate to its transynaptic function? Pikachurin and dystroglycan are proteins that are expressed in the extracellular space of the cone to BC synapse and are required for the structural integrity of the synapse (Omori et al., 2012). Dystroglycan is anchored in the membrane of the cone terminal and pikachurin likely binds dystroglycan. The binding partner of pikachurin on the $\mathrm{BC}$ side is unknown. Both proteins are present in the Lrit3 $^{-}$. I propose 
using the LRIT3 antibody to determine if it is expressed in the pikachurin ko mouse.

3. Is LRIT3 regulating the structural integrity of the synapse? We have shown that cone terminals express ribbons, and form contacts with both ON and OFF BCs. Techniques such as block face EM to create 3D reconstructions of the OPL in Lrit3\% would be useful to investigate the synaptic structure.

4. What is the PNA binding protein at the cone terminal? LRIT3 may regulate proteins whose functions shape glutamate transmission. Given the downregulation of PNA binding protein in the Lrit3 $^{--}$, that protein may have such a function. We have designed experiments to identify the protein. Proteins pulled down from retinal lysate with PNA will be identified using mass spectrometry. PNA binds proteins in the outer segments and terminals of the cones. Only the protein at the cone terminal is downregulated in the Lrit3 $^{-}$. Therefore, we plan to perform the mass spectrometry from WT and Lrit3\%- isolated terminal fractions.

\section{Conclusions}

My work has clarified the role of two of these channels in shaping visual responses. I have shown that TRPV1 modulates visual signal processing in the inner retina to enhance the sensitivity to light and match the timing of the responses to the stimulus. Has implications in enhancing the perception of fine 
details. The function of TRPM1 in ON pathway signaling has been well established. We further characterized aspects of the ON BC signalplex. Specifically, we showed two extracellular proteins are necessary for localization for TRPM1 in the rod and cone BCs. We further show the importance of one of these proteins, LRIT3, in trans-synaptic function of cones. With these understandings of the role of these proteins in shaping the function of specific neuronal pathways, future work can be conducted to establish the mechanisms behind it. 


\section{REFERENCES}

Ahmad, K.M., Klug, K., Herr, S., Sterling, P., Schein, S. (2003) Cell density ratios in a foveal patch in macaque retina. Vis. Neurosci. 20:189-209

Altimus, C. M., Guler, A. D., Alam, N. M., Arman, A. C., Prusky, G. T., Sampath, A. P., \& Hattar, S. (2010). Rod photoreceptors drive circadian photoentrainment across a wide range of light intensities. Nat Neurosci, 13(9), 1107-1112. doi:10.1038/nn.2617

Anderson, J.R., Jones, B.W., Watt, C.B., Shaw, M.V., Yang, J.H., et al., (2011) Exploring the retinal connectome. Mol Vis 17:355-379

Anishchenko, A., Greschner, M., Elstrott, J., Sher, A., Litke, A.M., Feller, M.B. \& Chichilnisky, E.J. (2010) Receptive field mosaics of retinal ganglion cells are established without visual experience. J Neurophysiol 103:1856-64

Athanasiou, A., Smith, P.A., Vakilpour, S., Kumaran, N.M., Turner, A.E., Bagiokou, D., et al. (2007). Vanilloid receptor agonists and antagonists are mitochondrial inhibitors: How vanilloids cause non-vanilloid receptor mediated cell death. Biochem \& Biophys. Research Communications 354(1):50-55.

Audo, I., Bujakowska, K., Orhan, E., Poloschek, C. M., Defoort-Dhellemmes, S., Drumare, I., ... Zeitz, C. (2012). Whole-exome sequencing identifies mutations in GPR179 leading to autosomal-recessive complete congenital stationary night blindness. Am J Hum Genet, 90(2), 321-330. doi:10.1016/j.ajhg.2011.12.007

Audo, I., Kohl, S., Leroy, B. P., Munier, F. L., Guillonneau, X., Mohand-Said, S., . . Zeitz, C. (2009). TRPM1 is mutated in patients with autosomalrecessive complete congenital stationary night blindness. Am J Hum Genet, 85(5), 720-729. doi:10.1016/j.ajhg.2009.10.013

Awatramani, G.B. \& Slaughter, M.M. (2000) Origin of transient and sustained responses in ganglion cells of the retina. J. of Neuroscience 20(18):70877095.

Badea \& Nathans (2004). Quantitative analysis of neuronal morphologies in the mouse retina visualized by using a genetically directed reporter. $J$ of Comparative Neurology 480:331-351.

Baden, T., Berens, P., Bethge, M., \& Euler, T. (2013). Spikes in mammalian bipolar cells support temporal layering of the inner retina. Curr Biol, 23(1), 48-52. doi:10.1016/j.cub.2012.11.006

Barlow, H. B. (1953) Summation and inhibition in the frog's retina. J Physiol 119:69-88

Barlow, H. B., \& Levick, W. R. (1969). Changes in the maintained discharge with adaptation level in the cat retina. J Physiol, 202(3), 699-718. 
Bauer, C.A., Brozoski, T.J. \& Myers, K.S. (2007). Acoustic injury and TRPV1 expression in the cochlear spiral ganglion. The International Tinnitus Journal 13(1):21-28.

Baver, S. B., Pickard, G. E., Sollars, P. J., \& Pickard, G. E. (2008). Two types of melanopsin retinal ganglion cell differentially innervate the hypothalamic suprachiasmatic nucleus and the olivary pretectal nucleus. Eur $\mathrm{J}$ Neurosci, 27(7), 1763-1770. doi:10.1111/j.1460-9568.2008.06149.x

Baylor, D. A., Fuortes, M. G. F., \& O'Bryan, P. M. (1971). Receptive fields of cones in the retina of the turtle. J Physiol, 214(2), 265-294. doi:10.1113/jphysiol.1971.sp009432

Bellone, R.R., Brooks, S.A., Sandmeyer, L., Murphy, B.A., Forsyth, G., Archer, S., Bailey, E. \& Grahn, B. (2008) Differential gene expression of TRPM1, the potential cause of congenital stationary night blindness and coat spotting patterns (LP) in the Appaloosa horse (Equus caballus). Genetics 179:1861-1870

Berson, D. M., Castrucci, A. M., \& Provencio, I. (2010). Morphology and mosaics of melanopsin-expressing retinal ganglion cell types in mice. J Comp Neurol, 518(13), 2405-2422. doi:10.1002/cne.22381

Berson, D. M., Dunn, F. A., \& Takao, M. (2002). Phototransduction by retinal ganglion cells that set the circadian clock. Science, 295(5557), 1070-1073. doi:10.1126/science.1067262

Bevan S, Szolcsanyi J. (1990). Sensory neuronspecific actions of capsaicin: mechanisms and applications. Trends Pharmacol. Sci 11:330-33

Blainey, P., Krzywinski, M., \& Altman, N. (2014). Points of significance: replication. Nature methods, 11(9), 879-880.

Blasiak, A., Blasiak, T., Lewandowski, M. H., Hossain, M. A., Wade, J. D., \& Gundlach, A. L. (2013). Relaxin-3 innervation of the intergeniculate leaflet of the rat thalamus - neuronal tract-tracing and in vitro electrophysiological studies. Eur J Neurosci, 37(8), 1284-1294. doi:10.1111/ejn.12155

Bloomfield, S. A., \& Dacheux, R. F. (2001). Rod vision: pathways and processing in the mammalian retina. Prog Retin Eye Res, 20(3), 351-384.

Bloomfield, S. A., \& Völgyi, B. (2009). The diverse functional roles and regulation of neuronal gap junctions in the retina. Nat Rev Neurosci, 10(7), 495-506.

Bloomfield, S. A., Xin, D., \& Osborne, T. (1997). Light-induced modulation of coupling between All amacrine cells in the rabbit retina. Vis Neurosci, 14(3), 565-576.

Bhaskaran, M.D, \& Smith, B.N. (2010) Cannabinoid-mediated inhibition of recurrent excitatory circuitry in the dentate gyrus in a mouse model of temporal lobe epilepsy. PLOS ONE 5(5):e10683

Borghuis, B. G., Looger, L. L., Tomita, S., \& Demb, J. B. (2014). Kainate receptors mediate signaling in both transient and sustained OFF bipolar cell pathways in mouse retina. $J$ Neurosci, 34(18), 6128-6139. doi:10.1523/jneurosci.4941-13.2014

Borghuis, B. G., Marvin, J. S., Looger, L. L., \& Demb, J. B. (2013). Two-photon imaging of nonlinear glutamate release dynamics at bipolar cell synapses 
in the mouse retina. J Neurosci, 33(27), 10972-10985.

doi:10.1523/jneurosci.1241-13.2013

Borghuis, B. G., Tian, L., Xu, Y., Nikonov, S. S., Vardi, N., Zemelman, B. V., \& Looger, L. L. (2011). Imaging light responses of targeted neuron populations in the rodent retina. $J$ Neurosci, 31(8), 2855-2867. doi:10.1523/jneurosci.6064-10.2011

Bornschein, H., \& Schubert, G. (1952). [Electromyography of rotatory nystagmus]. Wien $Z$ Nervenheilkd Grenzgeb, 5(2-3), 149-154.

Boycott, B.B., and Wässle, H. (1974). The morphological types of ganglion cells of the domestic cat's retina. J Physiol 240, 397-419.

Boycott, K. M., Sauve, Y., \& MacDonald, I. M. (1993). X-Linked Congenital Stationary Night Blindness. In R. A. Pagon, M. P. Adam, H. H. Ardinger, S. E. Wallace, A. Amemiya, L. J. H. Bean, T. D. Bird, C. T. Fong, H. C. Mefford, R. J. H. Smith, \& K. Stephens (Eds.), GeneReviews(R). Seattle (WA): University of Washington, Seattle

University of Washington, Seattle. All rights reserved.

Buffelli, M., Burgess, R. W., Feng, G., Lobe, C. G., Lichtman, J. W., \& Sanes, J. R. (2003). Genetic evidence that relative synaptic efficacy biases the outcome of synaptic competition. Nature, 424(6947), 430-434. doi:10.1038/nature01844

Cantrell, D.R., Cang, J., Troy, J.B. \& Liu, X. (2010) Non-centered spike-triggered covariance analysis reveals neurotrophin-3 as a developmental regulator of receptive field properties of ON-OFF retinal ganglion cells. PLOS Computational Biol 6(10): e1000967.

Cao, Y., Pahlberg, J., Sarria, I., Kamasawa, N., Sampath, A.P., Martemyanov, K.A. (2012). Regulation of G protein signaling RGS7 and RGS11 determine the onset of the light response in ON bipolar neurons. Proc Natl Acad Sci U S A. 109:7905-7910

Carr, R. E. (1974). Congenital stationary nightblindness. Trans Am Ophthalmol Soc, 72, 448-487.

Castillo, P. E., Younts, T. J., Chávez, A. E., \& Hashimotodani, Y. (2012). Endocannabinoid signaling and synaptic function. Neuron, 76(1), 70-81. doi:10.1016/j.neuron.2012.09.020

Caterina, M.J. \& Julius, D. (2001). The vanilloid receptor: a molecular gateway to the pain pathway. Annu. Rev. Neurosci. 24:487-517

Caterina, M. J., Leffler, A., Malmberg, A. B., Martin, W. J., Trafton, J., PetersenZeitz, K. R., . . Julius, D. (2000). Impaired nociception and pain sensation in mice lacking the capsaicin receptor. Science, 288(5464), 306-313.

Caterina, M. J., Schumacher, M. A., Tominaga, M., Rosen, T. A., Levine, J. D., \& Julius, D. (1997). The capsaicin receptor: a heat-activated ion channel in the pain pathway. Nature, 389(6653), 816-824. doi:10.1038/39807

Carter-Dawson, L.D. \& LaVail, M.M. (1979) Rods and cones in the mouse retina. I. Structural analysis using light and electron microscopy. J Comp Neurol 188(2):245-62.

Cavanaugh, D.J., Chesler, A.T., Braz, J.M., Shah, N.M., Julius, D. \& Basbaum, A.I. (2011a). Restriction of transient receptor potential vanilloid-1 to the 
peptidergic subset of primary afferent neurons follows its developmental downregulation in nonpeptidergic neurons. J Neuroscience 31(28):

10119-10127.

Cavanaugh, D.J., Chesler, A.T., Jackson, A.C., Sigal, Y.M., Yamanaka, H., Grant, R., et al. (2011b). Trpv1 reporter mice reveal highly restricted brain distribution and functional expression in arteriolar smooth muscle cells. $J$ Neuroscience 31(13): 5067-5077

Chabre, M. (1985) Trigger and amplification mechanisms in visual phototransduction. Annu Rev Biophys Chem 14: 331-360.

Chavez, A.E., Chiu, C.Q. \& Castillo, P.E. (2010) TRPV1 activation by endogenous anandamide triggers postsynaptic long-term depression in dentate gyrus. Nature Neuroscience 13: 1511-1518.

Chen, H., Zhao, Y., Liu, M., Feng, L., Puyang, Z., Yi, J., . . Liu, X. (2015). Progressive degeneration of retinal and superior collicular functions in mice with sustained ocular hypertension. Invest Ophthalmol Vis Sci, 56(3), 1971-1984.

Chen, S. K., Badea, T. C., \& Hattar, S. (2011a). Photoentrainment and pupillary light reflex are mediated by distinct populations of ipRGCs. Nature, 476(7358), 92-95. doi:10.1038/nature10206

Chen, X., Hsueh, H.A., Greenberg, K. \& Werblin, F.S. (2010) Three forms of spatial temporal feedforward inhibition are common to different ganglion cell types in rabbit retina. J Neurophysiol 103:2618-32.

Chiao, C. C., \& Masland, R. H. (2003). Contextual tuning of direction-selective retinal ganglion cells. Nat Neurosci, 6(12), 1251-1252. doi:10.1038/nn1147

Chun, M.H., Han, S-H., chung, J-W., Wässle, H. (1993) Electron microscopic analysis of the rod pathway of the rat retina. J Comp Neurol. 332:421-432

Clapham, D.E., Nilius, B. \& Owsianik, G. (2012). Transient receptor potential channels. Last modified on 11/10/2012. Accessed on 20/12/2012. IUPHAR database.

Cook, P.B., and McReynolds, J.S. (1998). Modulation of sustained and transient lateral inhibitory mechanisms in the mudpuppy retina during light adaptation. J Neurophysiol 79, 197-204.

Coombs, J., Van der List, D., Wang, G.-Y. \& Chalupa, L.M. (2006). Morphological properties of mouse retinal ganglion cells. Neuroscience 140: 123-136.

Cosens, D.J. (1969) Abnormal electroretinogram from a Drosophila mutant. Nature 224: 285-287.

Costa, R. M., Liu, L., Nicolelis, M. A., \& Simon, S. A. (2005). Gustatory effects of capsaicin that are independent of TRPV1 receptors. Chem Senses, 30 Suppl 1, i198-200. doi:10.1093/chemse/bjh183

Crooks, J. and Kolb, H. (1992) Localization of GABA, glycine, glutamate, and tyrosine hydroxylase in the human retina. J Comp Neurol. 315(3): 287302

Curcio CA, Sloan KR, Kalina RE \& Hendrickson AE (1990). Human photoreceptor topography. J Comp Neurol. 292(4): 497-523 
Czeisler, C. A. (1995). The effect of light on the human circadian pacemaker. Ciba Found Symp, 183, 254-290; discussion 290-302.

Della Santina, L., Inman, D. M., Lupien, C. B., Horner, P. J., \& Wong, R. O. (2013). Differential progression of structural and functional alterations in distinct retinal ganglion cell types in a mouse model of glaucoma. $J$ Neurosci, 33(44), 17444-17457. doi: 10.1523/JNEUROSCI.5461-12.2013

DeltaBase Histology Atlas: Sense Organs: Retina 40x (2000-2006). Deltagen Inc. Retrieved from http://www.deltagen.com/target/histologyatlas/HistologyAtlas.html

DeVries, S.H. (2000) Bipolar cells use kainite and AMPA receptors to filter visual information into separate channels. Neuron 28(3): 847-856.

Dhande, O. S., Estevez, M. E., Quattrochi, L. E., El-Danaf, R. N., Nguyen, P. L., Berson, D. M., \& Huberman, A. D. (2013). Genetic dissection of retinal inputs to brainstem nuclei controlling image stabilization. J Neurosci, 33(45), 17797-17813. doi:10.1523/jneurosci.2778-13.2013

Dhingra, A., Jiang, M., Wang, T. L., Lyubarsky, A., Savchenko, A., Bar-Yehuda, T., ... Vardi, N. (2002). Light response of retinal ON bipolar cells requires a specific splice variant of Galpha(o). J Neurosci, 22(12), 4878-4884.

Dhingra A, Lyubarsky A, Jiang M, Pugh EN Jr, Birnbaumer L, Sterling P \& Vardi $\mathrm{N}(2000)$. The light response of $\mathrm{ON}$ bipolar neurons requires $\mathrm{Ga}_{\circ} . J$ Neurosci 20, 9053-9058.

Dhingra, A., Ramakrishnan, H., Neinstein, A., Fina, M. E., Xu, Y., Li, J., . . Vardi, N. (2012). Gbeta3 is required for normal light $O N$ responses and synaptic maintenance. J Neurosci, 32(33), 11343-11355. doi:10.1523/jneurosci.1436-12.2012

Dolan, R. P., \& Schiller, P. H. (1994). Effects of ON channel blockade with 2amino-4-phosphonobutyrate (APB) on brightness and contrast perception in monkeys. Vis Neurosci, 11(1), 23-32.

Dong, C.J., and Hare, W.A. (2003). Temporal modulation of scotopic visual signals by $\mathrm{A} 17$ amacrine cells in mammalian retina in vivo. $J$ Neurophysiol 89, 2159-2166.

Dong, C. \& Werblin, F.S. (1998). Temporal contrast enhancement via GABA feedback at bipolar terminals in the Tiger Salamander retina. American Physiological Society 1: 2171-2180

Dowling, J.E. (1987) The retina: an approachable part of the brain. Cambridge, Mass: Harvard University Press.

Dryja, T. P., McGee, T. L., Berson, E. L., Fishman, G. A., Sandberg, M. A., Alexander, K. R., ... Rajagopalan, A. S. (2005). Night blindness and abnormal cone electroretinogram $\mathrm{ON}$ responses in patients with mutations in the GRM6 gene encoding mGluR6. Proc Natl Acad Sci U S A, 102(13), 4884-4889. doi:10.1073/pnas.0501233102

Ecker, J. L., Dumitrescu, O. N., Wong, K. Y., Alam, N. M., Chen, S. K., LeGates, T., ... Hattar, S. (2010). Melanopsin-expressing retinal ganglion-cell photoreceptors: cellular diversity and role in pattern vision. Neuron, 67(1), 49-60. doi:10.1016/j.neuron.2010.05.023 
Edwards, J. G. (2014). TRPV1 in the central nervous system: synaptic plasticity, function, and pharmacological implications. Prog Drug Res, 68, 77-104.

Eggers, E. D., Klein, J. S., \& Moore-Dotson, J. M. (2013). Slow changes in $\mathrm{Ca} 2(+)$ cause prolonged release from GABAergic retinal amacrine cells. $J$ Neurophysiol, 110(3), 709-719. doi:10.1152/jn.00913.2012

Eggers, E. D., \& Lukasiewicz, P. D. (2006). GABA(A), GABA(C) and glycine receptor-mediated inhibition differentially affects light-evoked signalling from mouse retinal rod bipolar cells. J Physiol, 572(Pt 1), 215-225. doi:10.1113/jphysiol.2005.103648

Eggers, E.D., and Lukasiewicz, P.D. (2010). Interneuron circuits tune inhibition in retinal bipolar cells. JNeurophysiol 103, 25-37.

Eggers, E.D., and Lukasiewicz, P.D. (2011). Multiple pathways of inhibition shape bipolar cell responses in the retina. VisNeurosci 28, 95-108.

Eggers, E.D., McCall, M.A., and Lukasiewicz, P.D. (2007). Presynaptic inhibition differentially shapes transmission in distinct circuits in the mouse retina. JPhysiol 582, 569-582.

Elstrott, J., Anishchenko, A., Greschner, M., Sher, A., Litke, A. M., Chichilnisky, E. J., \& Feller, M. B. (2008). Direction selectivity in the retina is established independent of visual experience and cholinergic retinal waves. Neuron, 58(4), 499-506. doi:10.1016/j.neuron.2008.03.013

Enroth-Cugell, C., and Robson, J.G. (1966). The contrast sensitivity of retinal ganglion cells of the cat. J Physiol 187, 517-552

Enroth-Cugell, C. \& Lennie, P. (1975) The control of retinal ganglion cell discharge by receptive field surrounds. J Physiol 247:551-578.

Estevez, M.E., Fogerson, P.M., Ildardi, M.C., Borghuis, B.G., Chan, E., Weng, S., et al. (2012). Form and function of the M4 cell, an intrinsically photosensitive retinal ganglion cell type contributing to geniculocortical vision. J. of Neuroscience 32(39): 13608-13620

Euler, T., Haverkamp, S., Schubert, T., \& Baden, T. (2014). Retinal bipolar cells: elementary building blocks of vision. Nature Reviews Neuroscience, 15(8), 507-519

Euler, T., H. Schneider, and H. Wässle, (1996) Glutamate responses of bipolar cells in a slice preparation of the rat retina. J Neurosci, 16(9): 2934-44.

Fain, G.L., Matthews, H.R., Cornwall, M.C. \& Koutalos, Y. (2001). Adaptation in vertebrate photoreceptors. Pharmalcological Review 81:117-15

Famiglietti, E.V. \& Kolb, H. (1975) A bistratified amacrine cell and synaptic circuitry in the inner plexiform layer of the retina. Brain Research 84:293300

Farajian, R., Pan, F., Akopian, A., Volgyi, B., \& Bloomfield, S. A. (2011). Masked excitatory crosstalk between the ON and OFF visual pathways in the mammalian retina. J Physiol, 589(Pt 18), 4473-4489.

Farrow, K., Teixeira, M., Szikra, T., Viney, T.J., Balint, K., Yonehara, K., and Roska, B. (2013). Ambient illumination toggles a neuronal circuit switch in the retina and visual perception at cone threshold. Neuron 78, 325338. 
Flores-Herr, N., Protti, D.A., Wässle, H. (2001) Synaptic currents generating the inhibitory surround of ganglion cellsin the mammalian retina. $J$ Neurosci 21:4852-4863

Foster, R. G., Provencio, I., Hudson, D., Fiske, S., De Grip, W., \& Menaker, M. (1991). Circadian photoreception in the retinally degenerate mouse $(\mathrm{rd} / \mathrm{rd})$. J Comp Physiol A, 169(1), 39-50.

Fransen, J. W., Pangeni, G., Pyle, I. S., \& McCall, M. A. (2015). Functional changes in $\mathrm{Tg} \mathrm{P} 23 \mathrm{H}-1$ rat retinal responses: differences between $\mathrm{ON}$ and OFF pathway transmission to the superior colliculus. J Neurophysiol, 114(4), 2368-2375. doi:10.1152/jn.00600.2015

Freed, M. A., Smith, R. G., \& Sterling, P. (2003). Timing of quantal release from the retinal bipolar terminal is regulated by a feedback circuit. Neuron, 38(1), 89-101.

$\mathrm{Fu}, \mathrm{Y}$ (2010) Phototransduction in Rods and Cones. Webvision Salt Lake City, Utah: John Moran Eye Center, University of Utahlast updated 4/1/2010.

Gasulla, J., Beltran Gonzalez, A. N., \& Calvo, D. J. (2012). Nitric oxide potentiation of the homomeric rho1 $\mathrm{GABA}(\mathrm{C})$ receptor function. $\mathrm{Br} \mathrm{J}$ Pharmacol, 167(6), 1369-1377.

Ghosh, K.K., Bujan, S., Haverkamp, S., Feigenspan, A., and Wässle, H. (2004). Types of bipolar cells in the mouse retina. J Comp Neurol 469: 70-82.

Gibson, H. E., Edwards, J. G., Page, R. S., Van Hook, M. J., \& Kauer, J. A. (2008). TRPV1 channels mediate long-term depression at synapses on hippocampal interneurons. Neuron, 57(5), 746-759. doi:10.1016/j.neuron.2007.12.027

Gilliam, J.C. \& Wensel, T.G. (2011). TRP channel gene expression in the mouse retina. Vision Research 51: 2440-2452

Gonzalez-Menendez, I., Reinhard, K., Tolivia, J., Wissinger, B., \& Munch, T. A. (2015). Influence of Opa1 Mutation on Survival and Function of Retinal Ganglion Cells. Invest Ophthalmol Vis Sci, 56(8), 4835-4845.

Gregg, R. G., Kamermans, M., Klooster, J., Lukasiewicz, P. D., Peachey, N. S., Vessey, K. A., \& McCall, M. A. (2007). Nyctalopin expression in retinal bipolar cells restores visual function in a mouse model of complete $\mathrm{X}$ linked congenital stationary night blindness. J Neurophysiol, 98(5), 30233033. doi:10.1152/jn.00608.2007

Gregg, R. G., Mukhopadhyay, S., Candille, S. I., Ball, S. L., Pardue, M. T., McCall, M. A., \& Peachey, N. S. (2003). Identification of the gene and the mutation responsible for the mouse nob phenotype. Invest Ophthalmol Vis Sci, 44(1), 378-384.

Greka, A., Navarro, B., Oancea, E., Duggan, A. and Clapham, D.E. (2003) TRPC5 is a regulator of hippocampal neurite length and growth cone morphology. Nat Neurosci 6:37-45

Grimes, W. N., Graves, L. R., Summers, M. T., \& Rieke, F. (2015). A simple retinal mechanism contributes to perceptual interactions between rod- and cone-mediated responses in primates. eLife, 4, e08033.

doi:10.7554/eLife.08033 
Grueter, B. A., Brasnjo, G. \& Malenka, R.C. (2010) Postsynaptic TRPV1 triggers cell type-specific long-term depression in the nucleus accumbens. Nature Neuroscience 13: 1519-1525

Guo, H., Carlson, J. A., \& Slominski, A. (2012). Role of TRPM in melanocytes and melanoma. Exp Dermatol, 21(9), 650-654. doi:10.1111/j.16000625.2012.01565.x

Hamby, A. M., Rosa, J. M., Hsu, C. H., \& Feller, M. B. (2015). CaV3.2 KO mice have altered retinal waves but normal direction selectivity. Vis Neurosci, 32(10).

Hargrave, P.A. \& McDowell, J.H.,(1992). Rhodopsin and phototransduction; a model system for G protein-linked receptors. FASEB J. 6(6):2323-2331

Hartline, H. K. (1940). THE EFFECTS OF SPATIAL SUMMATION IN THE RETINA ON THE EXCITATION OF THE FIBERS OF THE OPTIC NERVE. American Journal of Physiology -- Legacy Content, 130(4), 700711.

Hartveit, E. (1999) Reciprocal synaptic interactions between rod bipolar cells in the rat retina. J Neurophysiol. 81:2923-36.

Hatori, M., Le, H., Vollmers, C., Keding, S. R., Tanaka, N., Buch, T., . . Panda, S. (2008). Inducible ablation of melanopsin-expressing retinal ganglion cells reveals their central role in non-image forming visual responses. PLoS One, 3(6), e2451. doi:10.1371/journal.pone.0002451

Hatori, M., \& Panda, S. (2010). The emerging roles of melanopsin in behavioral adaptation to light. Trends Mol Med, 16(10), 435-446. doi:10.1016/j.molmed.2010.07.005

Hattar, S., Kumar, M., Park, A., Tong, P., Tung, J., Yau, K. W., \& Berson, D. M. (2006). Central projections of melanopsin-expressing retinal ganglion cells in the mouse. J Comp Neurol, 497(3), 326-349. doi:10.1002/cne.20970

Haverkamp, S., Muller, U., Zeilhofer, H.U., Harvey, R.J., and Wässle, H. (2004). Diversity of glycine receptors in the mouse retina: localization of the alpha2 subunit. J Comp Neurol 477, 399-411.

Haverkamp, S. \& Wässle, H. (2000). Immunocytochemical analysis of the mouse retina. J.of Comparative Neurology 424:1-23

Haverkamp, S., \& Wässle, H. (2004). Characterization of an amacrine cell type of the mammalian retina immunoreactive for vesicular glutamate transporter 3. J Comp Neurol, 468(2), 251-263.

Hecht, S., Shlaer, S. \& Pirenne, M.H. (1942). Energy, quanta, and vision. J of General Physiol. 25(6):819-840

Herrmann, R., Heflin, S. J., Hammond, T., Lee, B., Wang, J., Gainetdinov, R. R., ... Arshavsky, V. Y. (2011). Rod vision is controlled by dopaminedependent sensitization of rod bipolar cells by GABA. Neuron, 72(1), 101110

Herzog, E. D. (2007). Neurons and networks in daily rhythms. Nat Rev Neurosci, 8(10), 790-802. doi:10.1038/nrn2215

Hippenmeyer, S., Vrieseling, E., Sigrist, M., Portmann, T., Laengle, C., Ladle, D. R., \& Arber, S. (2005). A developmental switch in the response of DRG 
neurons to ETS transcription factor signaling. PLoS Biol, 3(5), e159. doi:10.1371/journal.pbio.0030159

Ho, K.W., Ward, N.J. \& Calkins, J. (2012) TRPV1: a stress response protein in the central nervous system. Am J Neurodegener Dis. 1(1): 1-14

Holzer P. (1991) Capsaicin: cellular targets, mechanisms of action, and selectivity for thin sensory neurons. Pharmacol. Rev. 43:143-201

Hong, Y.K., Kim, I.-J. \& Sanes, J.R. (2011). Stereotyped axonal arbors of retinal ganglion cell subsets in the mouse superior colliculus. J. Comparative Neurology 519(9): 1691-171

Hu, Y., Gu, Q., Lin, R. L., Kryscio, R., \& Lee, L. Y. (2010). Calcium transient evoked by TRPV1 activators is enhanced by tumor necrosis factor-\{alpha\} in rat pulmonary sensory neurons. Am J Physiol Lung Cell Mol Physiol, 299(4), L483-492. doi:10.1152/ajplung.00111.2010

Hughes, S., Jagannath, A., Rodgers, J., Hankins, M. W., Peirson, S. N., \& Foster, R. G. (2016). Signalling by melanopsin (OPN4) expressing photosensitive retinal ganglion cells. Eye (Lond), 30(2), 247-254.

Hwang, S. J., Oh, J. M., \& Valtschanoff, J. G. (2005). Expression of the vanilloid receptor TRPV1 in rat dorsal root ganglion neurons supports different roles of the receptor in visceral and cutaneous afferents. Brain Res, 1047(2), 261-266. doi:10.1016/j.brainres.2005.04.036

Ichinose, T., \& Lukasiewicz, P. D. (2002). GABA transporters regulate inhibition in the retina by limiting $\mathrm{GABA}(\mathrm{C})$ receptor activation. $J$ Neurosci, 22(8), 3285-3292. doi:20026320

Ikeda, H. \& Wright, M.J. (1972) Receptive field organization of "sustained" and "transient" retinal ganglion cells which subserve different function roles. $J$ Physiol. 227(3): 769-800.

Ivanova, E., Muller, U., and Wässle, H. (2006). Characterization of the glycinergic input to bipolar cells of the mouse retina. EurJNeurosci 23, 350-364.

Jackson, C. R., Capozzi, M., Dai, H., \& McMahon, D. G. (2014). Circadian perinatal photoperiod has enduring effects on retinal dopamine and visual function. J Neurosci, 34(13), 4627-4633. doi:10.1523/jneurosci.488713.2014

Jaubert-Miazza, L., Green, E., Lo, F. S., Bui, K., Mills, J., \& Guido, W. (2005). Structural and functional composition of the developing retinogeniculate pathway in the mouse. Vis Neurosci, 22(5), 661-676.

doi:10.1017/s0952523805225154

Jeffrey, B. G., Morgans, C. W., Puthussery, T., Wensel, T. G., Burke, N. S., Brown, R. L., \& Duvoisin, R. M. (2010). R9AP stabilizes RGS11-G beta5 and accelerates the early light response of ON-bipolar cells. Vis Neurosci, 27(1-2), 9-17. doi:10.1017/s0952523809990319

Jeon C-J., Strettoi E. \& Masland R.H. (1998). The major cell populations of the mouse retina. $J$ Neurosci 18:8936-8946.

Karai, L. J., Russell, J. T., Iadarola, M. J., \& Olah, Z. (2004). Vanilloid receptor 1 regulates multiple calcium compartments and contributes to $\mathrm{Ca} 2+$-induced Ca2+ release in sensory neurons. J Biol Chem, 279(16), 16377-16387. doi:10.1074/jbc.M310891200 
Kawahara, H., Drew, G. M., Christie, M. J., \& Vaughan, C. W. (2011). Inhibition of fatty acid amide hydrolase unmasks CB1 receptor and TRPV1 channelmediated modulation of glutamatergic synaptic transmission in midbrain periaqueductal grey. Br J Pharmacol, 163(6), 1214-1222. doi:10.1111/j.1476-5381.2010.01157.x

Kay, J.N., De la Huerta, I., Kim, I.J., Zhang, Y., Yamagata, M., Chu, M.W., Meister, M., and Sanes, J.R. (2011). Retinal ganglion cells with distinct directional preferences differ in molecular identity, structure, and central projections. J Neurosci 31, 7753-7762.

Kim, C. S., Kawada, T., Kim, B. S., Han, I. S., Choe, S. Y., Kurata, T., \& Yu, R. (2003). Capsaicin exhibits anti-inflammatory property by inhibiting IkB-a degradation in LPS-stimulated peritoneal macrophages. Cell Signal, 15(3), 299-306.

Kim, I.B., Lee, M.Y., Oh, S.J., Kim, K.Y. \& Chun, M.H (1998) Double labeling techniques demonstrate that rod bipolar cells are under GABAergic control in the inner plexiform layer of the rat retina. Cell Tissue Res 292: 17-25.

Kim, I.J., Zhang, Y., Meister, M., and Sanes, J.R. (2010). Laminar restriction of retinal ganglion cell dendrites and axons: subtype-specific developmental patterns revealed with transgenic markers. J Neurosci 30, 1452-1462.

Kim, T., Soto, F., \& Kerschensteiner, D. (2015). An excitatory amacrine cell detects object motion and provides feature-selective input to ganglion cells in the mouse retina. eLife, 4. doi:10.7554/eLife.08025

Koehler, C.L., Akimov, N.P. \& Renteria, R.C. (2011) Receptive field center size decreases and firing properties mature in ON and OFF retinal ganglion cells after eye opening in the mouse. J Neurophysiol 106(2):895-904.

Koike, C., Obara, T., Uriu, Y., Numata, T., Sanuki, R., Miyata, K., Koyasu, T., Ueno, S., Funabiki, K., Tani, A., et al. (2010). TRPM1 is a component of the retinal ON bipolar cell transduction channel in the mGluR6 cascade. Proceedings of the National Academy of Sciences of the United States of America 107, 332-337.

Kolb, H. \& Famiglietti, E.V. (1974) Rod and cone pathways in the inner plexiform layer of the cat retina. Science. 186: 47-49.

Kolb, H., \& Nelson, R. (1983). Rod pathways in the retina of the cat. Vision Res, 23(4), 301-312.

Kolb, H., Nelson, R \& Mariana. A. (1981). Amacrine cells, bipolar cells and ganglion cells of the cat retina: a Golgi study. Vis. Research 21:10811114.

Kolb, H., Fernandez, E., \& Nelson, R. (2003). Webvision: The neural organization of the vertebrate retina. Salt Lake City, Utah: John Moran Eye Center, University of Utah. Last updated Oct. 2010.

Kolb, H. (2011) Simple anatomy of the retina. Webvision. http://webvision.med.utah.edu/book/part-ii-anatomy-and-physiology-ofthe-retina/photoreceptors/ Salt Lake City, Utah: John Moran Eye Center, University of Utah. Last updated May 2012. 
Kong, J-H., Fish, D.R., Rockhill, R.L. \& Masland, R.H. (2005). Diversity of ganglion cells in the mouse retina: unsupervised morphological classification and its limits. J. of Comparative Neurology 489(3):293-310

Kothmann, W. W., Massey, S. C., \& O'Brien, J. (2009). Dopamine-stimulated dephosphorylation of connexin 36 mediates All amacrine cell uncoupling. J Neurosci, 29(47), 14903-14911.

Krassas, G. E., Tzotzas, T., Papazisis, K., Pazaitou-Panayiotou, K., \& Boboridis, K. (2007). The efficacy of somatostatin analogues in the treatment of diabetic retinopathy and thyroid eye disease. Clin Ophthalmol, 1(3), 209215.

Krauzlis, R. J., Lovejoy, L. P., \& Zenon, A. (2013). Superior colliculus and visual spatial attention. Annu Rev Neurosci, 36, 165-182. doi:10.1146/annurevneuro-062012-170249

Krishnaswamy, A., Yamagata, M., Duan, X., Hong, Y. K., \& Sanes, J. R. (2015). Sidekick 2 directs formation of a retinal circuit that detects differential motion. Nature, 524(7566), 466-470. doi:10.1038/nature14682

Križaj, D. (2012) Calcium stores in vertebrate photoreceptors. Adv Exp Med Biol 740:873-89

Krzywinski, M., \& Altman, N. (2014a). Points of significance: Comparing samples_part I. Nat Methods, 11(3), 215-216. doi: 10.1038/nmeth.2858

Krzywinski, M., \& Altman, N. (2014b). Points of significance: Comparing samples—part II. Nat Methods, 11(4), 355-356. doi: 10.1038/nmeth.2900

Kubova, Z., Kremlacek, J., Kuba, M., Chlubnova, J., \& Sverak, J. (2004). Photopic and scotopic VEPs in patients with congenital stationary nightblindness. Doc Ophthalmol, 109(1), 9-15.

Kuffler, S.W. (1953). Discharge patterns and functional organization of mammalian retina. J Neurophysiol 16, 37-68.

Lee, S., Chen, L., Chen, M., Ye, M., Seal, R. P., \& Zhou, Z. J. (2014). An unconventional glutamatergic circuit in the retina formed by vGluT3 amacrine cells. Neuron, 84(4), 708-715. doi:10.1016/j.neuron.2014.10.021

Lee, S. H., Ledri, M., Toth, B., Marchionni, I., Henstridge, C. M., Dudok, B., . . . Katona, I. (2015). Multiple Forms of Endocannabinoid and Endovanilloid Signaling Regulate the Tonic Control of GABA Release. J Neurosci, 35(27), 10039-10057. doi:10.1523/jneurosci.4112-14.2015

Lee, S., Zhang, Y., Chen, M., \& Zhou, Z. J. (2016). Segregated GlycineGlutamate Co-transmission from vGluT3 Amacrine Cells to ContrastSuppressed and Contrast-Enhanced Retinal Circuits. Neuron, 90(1), 2734.

Leonelli, M., Martins, D.O. \& Britto, L.R.G. (2010). TRPV1 receptors are involved in protein nitration and Muller cell reaction in the acutely axotomized rat retina. Experimental Eye Research 91(5):755-768.

Leonelli, M., Martins, D. O., \& Britto, L. R. (2013). Retinal cell death induced by TRPV1 activation involves NMDA signaling and upregulation of nitric oxide synthases. Cell Mol Neurobiol, 33(3), 379-392. 
Leonelli, M., Martins, D.O., Kihara, A.H. \& Britto, L.R.G. (2009). Ontogenetic expression of the vanilloid receptors TRPV1 and TRPV2 in the rat retina. International Journal of Developmental Neuroscience. 27: 709-718.

Li, P. H., Gauthier, J. L., Schiff, M., Sher, A., Ahn, D., Field, G. D., . . Chichilnisky, E. J. (2015). Anatomical identification of extracellularly recorded cells in large-scale multielectrode recordings. J Neurosci, 35(11), 4663-4675.

Liang, Z. \& Freed, M.A. (2010) The ON pathway rectifies the OFF pathway in the mammalian retina. $J$ Neurosci 30(16): 5533-43

Lin, B. \& Masland, R.H. (2006) Populations of wide-field amacrine cells in the mouse retina. J Comp Neurol 499:797-809

Liu, B., Zhang, C., Qin, F. (2005) Functional recovery from desensitization of vanilloid receptor TRPV1 requires resynthesis of phosphatidylinositol 4,5bisphosphate. J Neurosci. 25(19):4835-43

Long, D.J., Devantier, H.R., Brennan, F.X., Bryant, R.W., Salemme, F.R. \& Palmer, R.K. (2010). Pharmacologic antagonism of the oral aversive taste-directed response to capsaicin in a mouse brief access taste aversion assay. $J$ of Pharmacology and Experimental Therapeutics 332: 525-530.

Lukasiewicz, P. D., Eggers, E. D., Sagdullaev, B. T., \& McCall, M. A. (2004). $\mathrm{GABA}_{c}$ receptor-mediated inhibition in the retina. Vision Res, 44(28), 32893296. doi:10.1016/j.visres.2004.07.023

Lukasiewicz, P.D., Lawrence, J.E. \& Valentino, T.L. (1995) Desensitizing glutamate receptors shape excitatory synaptic inputs to tiger salamander retinal ganglion cells. The Journal of Neuroscience, 15(9): 6189-6199.

Lukasiewicz, P.D. \& Shields, C.R. (1998). A diversity of GABA receptors in the retina. Seminars in Cell \& Devel. Biology 9(3): 293-299.

Lukasiewicz, P.D., \& Werblin, F.S. (1990). The spatial distribution of excitatory and inhibitory inputs to ganglion cell dendrites in the tiger salamander retina. $J$ Neurosci 10, 210-221.

Lukasiewicz, P.D., \& Werblin, F.S. (1994). A novel GABA receptor modulates synaptic transmission from bipolar to ganglion and amacrine cells in the tiger salamander retina. J Neurosci 14, 1213-1223.

Lyubarsky, A. L., Daniele, L. L., \& Pugh, E. N., Jr. (2004). From candelas to photoisomerizations in the mouse eye by rhodopsin bleaching in situ and the light-rearing dependence of the major components of the mouse ERG. Vision Res, 44(28), 3235-3251.

Lyubarsky, A. L., \& Pugh, E. N., Jr. (1996). Recovery phase of the murine rod photoresponse reconstructed from electroretinographic recordings. $J$ Neurosci, 16(2), 563-571.

MacNeil, M.A., Heussy, J.K., Dacheux, R.F., Raviola, E., and Masland, R.H. (1999). The shapes and numbers of amacrine cells: matching of photofilled with Golgi-stained cells in the rabbit retina and comparison with other mammalian species. J Comp Neurol 413: 305-326.

Maddox, D. M., Vessey, K. A., Yarbrough, G. L., Invergo, B. M., Cantrell, D. R., Inayat, S., . . McCall, M. A. (2008). Allelic variance between GRM6 
mutants, Grm6nob3 and Grm6nob4 results in differences in retinal ganglion cell visual responses. J Physiol, 586(18), 4409-4424.

doi:10.1113/jphysiol.2008.157289

Madisen, L., Zwingman, T. A., Sunkin, S. M., Oh, S. W., Zariwala, H. A., Gu, H., . .. Zeng, H. (2010). A robust and high-throughput Cre reporting and characterization system for the whole mouse brain. Nat Neurosci, 13(1), 133-140. doi:10.1038/nn.2467

Maione, S., Cristino, L., Migliozzi, A.L., Georgiou, A.L., Starowicz, K., Salt, T.E. \& Di Marzo, V. (2009) TRPV1 channels control synaptic plasticity in the developing superior colliculus. J. Physiol. 587(11): 2521-2535.

Mandadi, S., Tominaga, T., Numazaki, M., Murayama, N., Saito, N., Armati, P.J., Roufogalis, B.D. \& Tominaga, M. (2004) Increased sensitivity of desensitized TRPV1 by PMA occurs through PKCepsilon-mediated phosphorylation at S800. Pain 123:103-116.

Manookin, M.B., Beaudoin, D.L., Ernst, Z.R., Flagel, L.J. \& Demb, J.B. (2008) Disinhibition combines with excitation to extend the operating range of the OFF visual pathway in daylight. J Neurosci 28:4136-4150

Marc, R.E., Jones, B.W., Watt, C.B., Anderson, J.R., Sigulinsky, C., and Lauritzen, S. (2013). Retinal connectomics: towards complete, accurate networks. Progress in retinal and eye research 37, 141-162.

Marc, R. E., Murry, R. F., \& Basinger, S. F. (1995). Pattern recognition of amino acid signatures in retinal neurons. J Neurosci, $15(7 \mathrm{Pt} 2), 5106-5129$.

Margolis, D.J., and Detwiler, P.B. (2007). Different mechanisms generate maintained activity in ON and OFF retinal ganglion cells. J Neurosci 27 , 5994-6005.

Marre, O., Amodei, D., Deshmukh, N., Sadeghi, K., Soo, F., Holy, T. E., \& Berry, M. J., 2nd. (2012). Mapping a complete neural population in the retina. $J$ Neurosci, 32(43), 14859-14873.

Martenson, M. E., Halawa, O. I., Tonsfeldt, K. J., Maxwell, C. A., Hammack, N., Mist, S. D., . . Heinricher, M. M. (2016). A possible neural mechanism for photosensitivity in chronic pain. Pain, 157(4), 868-878. doi:10.1097/j.pain.0000000000000450

Martinez-Garcia, M. C., Martinez, T., Paneda, C., Gallego, P., Jimenez, A. I., \& Merayo, J. (2013). Differential expression and localization of transient receptor potential vanilloid 1 in rabbit and human eyes. Histol Histopathol, 28(11), 1507-1516.

Masland, R. H. (2001) The fundamental plan of the retina. Nature Neuroscience 4: $877-886$.

Masland, R. H. (2012). The neuronal organization of the retina. Neuron, 76(2), 266-280. doi:10.1016/j.neuron.2012.10.002

Massey, S. C., Redburn, D. A., \& Crawford, M. L. (1983). The effects of 2-amino4-phosphonobutyric acid (APB) on the ERG and ganglion cell discharge of rabbit retina. Vision Res, 23(12), 1607-1613.

Masu, M., Iwakabe, H., Tagawa, Y., Miyoshi, T., Yamashita, M., Fukuda, Y., Sasaki, H., Hiroi, K., Nakamura, Y., Shigemoto, R., et al. (1995). Specific 
deficit of the ON response in visual transmission by targeted disruption of the mGluR6 gene. Cell 80, 757-765.

May, P. J. (2006). The mammalian superior colliculus: laminar structure and connections. Prog Brain Res, 151, 321-378. doi:10.1016/s00796123(05)51011-2

McCall, M. A., \& Gregg, R. G. (2008). Comparisons of structural and functional abnormalities in mouse b-wave mutants. J Physiol, 586(18), 4385-4392. doi:10.1113/jphysiol.2008.159327

McCall, M.A., Lukasiewicz, P.D., Gregg, R.G., and Peachey, N.S. (2002). Elimination of the rho1 subunit abolishes $\mathrm{GABA}(\mathrm{C})$ receptor expression and alters visual processing in the mouse retina. J Neurosci 22: 41634174.

Medvedeva, Y.V., Kim, M. \& Usachev, Y.M. (2009). Mechanisms of prolonged presynaptic $\mathrm{Ca} 2+$ signaling and glutamate release induced by TRPV1 activation in rat sensory neurons. J. of Neuroscience 28(20): 5295-5311.

Meng, J, Ovsepian, S.V., Wang, J. Pickering, M., Sasse, A. et al. (2009) Activation of TRPV1 mediates calcitonin gene-related peptide release, which excites trigeminal sensory neurons and is attenuated by a retargeted botulinum toxin with anti-nociceptive potential. $J$ Neurosci. 29(15): 4981-92.

Menger, N., Pow, D.V. \& Wässle (1998) Glycinergic amacrine cells of the rat retina. J Comp Neurol 401:34-46.

Merighi, A., Raviola, E., \& Dacheux, R. F. (1996). Connections of two types of flat cone bipolars in the rabbit retina. $J$ Comp Neurol, 371(1), 164-178. doi:10.1002/(SICI)1096-9861(19960715)371:1\&lt;164::AID-

CNE10\&gt;3.0.CO;2-S

Misgeld, T., Kerschensteiner, M., Bareyre, F. M., Burgess, R. W., \& Lichtman, J. W. (2007). Imaging axonal transport of mitochondria in vivo. Nat Meth, 4(7), 559-561.

Mishra, S.K., Tisel, S.M., Orestes, P., Bhangoo, S.K. \& Hoon, M.A. (2011). TRPV1-lineage neurons are required for thermal sensation. The European Molecular Biology Organization Journal 30: 582-593

Miyake, Y., Horiguchi, M., Ota, I., \& Shiroyama, N. (1987). Characteristic ERGflicker anomaly in incomplete congenital stationary night blindness. Invest Ophthalmol Vis Sci, 28(11), 1816-1823.

Miyake, Y., Yagasaki, K., Horiguchi, M., Kawase, Y., \& Kanda, T. (1986). Congenital stationary night blindness with negative electroretinogram. A new classification. Arch Ophthalmol, 104(7), 1013-1020.

Mohapatra, D.P. \& Nau, C. (2005) Regulation of Ca2+-dependent desensitization in the vanilloid receptor TRPV1 by calcineurin and cAMP-dependent protein kinase. J Biol Chem 280: 13424-32

Moore-Dotson, J. M., Klein, J. S., Mazade, R. E., \& Eggers, E. D. (2015). Different types of retinal inhibition have distinct neurotransmitter release properties. J Neurophysiol, 113(7), 2078-2090. doi:10.1152/jn.00447.2014

Moran, M.M., Xu, H. \& Clapham, D.E. (2004) TRP ion channels in the nervous system. Current Opinion in Neurobiol 14(3):362-369 
Morgan, J.L., Schubert, T. \& Wong, R.O.L (2008) Developmental patterning of glutamatergic synapses onto retinal ganglion cells. Neural Development $3(8): 1-19$

Moreira, F. A., Aguiar, D. C., Terzian, A. L., Guimaraes, F. S., \& Wotjak, C. T. (2012). Cannabinoid type 1 receptors and transient receptor potential vanilloid type 1 channels in fear and anxiety-two sides of one coin? Neuroscience, 204, 186-192. doi:10.1016/j.neuroscience.2011.08.046

Morgans, C.W., Zhang, J., Jeffrey, B.G., Nelson, S.M., Burke, N.S., Duvoisin, R.M. \& Brown, R.L. (2009). TRPM1 is required for the depolarizing light response in retinal ON-bipolar cells. Current Issue 106(45): 19174-19178

Mosinger, J.L., Yazulla, S. \& Studholme, K.M. (1986) GABA-like immunoreactivity in the vertebrate retina: a species comparison. Exp Eye Research 42:631-644.

Müller, L.P., Shelley, J. \& Weiler, R. (2007) Displaced amacrine cells of the mouse retina. $J$ of Comparative Neurology 505(2):177-189.

Murphy, G.J., and Rieke, F. (2008). Signals and noise in an inhibitory interneuron diverge to control activity in nearby retinal ganglion cells. Nature Neuroscience 11, 318-326.

Musella, A., De Chiara, V., Rossi, S., Prosperetti, C., Bernardi, G., et al.(2009) TRPV1 channels facilitate glutamate transmission in the striatum. Mol Cell Neurosci. 40(1): 89-97

Nakajima, Y., Iwakabe, H., Akazawa, C., Nawa, H., Shigemoto, R., Mizuno, N., \& Nakanishi, S. (1993). Molecular characterization of a novel retinal metabotropic glutamate receptor mGluR6 with a high agonist selectivity for L-2-amino-4-phosphonobutyrate. J Biol Chem, 268(16), 11868-11873.

Nakamura, M., Sanuki, R., Yasuma, T. R., Onishi, A., Nishiguchi, K. M., Koike, C., ... Furukawa, T. (2010). TRPM1 mutations are associated with the complete form of congenital stationary night blindness. Mol Vis, 16, 425437.

Nakashimo, Y., Takumida, M., Fukuiri, T., Anniko, M. \& Hirakawa, K. (2010). Expression of transient receptor potential channel vanilloid (TRPV) 1-4, melastin (TRPM) 5 and 8,and ankyrin (TRPA1) in the normal and methimazole-treated mouse olfactory epithelium. Acta Oto-Laryngologica 130: $1278-1286$

Nawy, S. (1999). The metabotropic receptor mGluR6 may signal through $\mathrm{G}(0)$, but not phosphodiesterase, in retinal bipolar cells. J Neurosci, 19(8), 29382944.

Nawy, S. (2004). Desensitization of the mGluR6 transduction current in tiger salamander On bipolar cells. J Physiol, 558(1), 137-146. doi:10.1113/jphysiol.2004.064980

Neuille, M., El Shamieh, S., Orhan, E., Michiels, C., Antonio, A., Lancelot, M. E., . . Zeitz, C. (2014). Lrit3 deficient mouse (nob6): a novel model of complete congenital stationary night blindness (cCSNB). PLoS One, 9(3), e90342. doi:10.1371/journal.pone.0090342

Neuille, M., Morgans, C. W., Cao, Y., Orhan, E., Michiels, C., Sahel, J. A., ... Zeitz, C. (2015). LRIT3 is essential to localize TRPM1 to the dendritic tips 
of depolarizing bipolar cells and may play a role in cone synapse formation. Eur J Neurosci, 42(3), 1966-1975. doi:10.1111/ejn.12959

Nobles, R.D., Zhang, C., Müller, U., Betz, H. and McCall, M.A. (2012). Selective Glycine Receptor a2 Subunit Control of Crossover Inhibition Between the On and Off Retinal Pathways. J Neurosci. 32(10):3321-3332

Nomura, A., Shigemoto, R., Nakamura, Y., Okamoto, N., Mizuno, N., \& Nakanishi, S. (1994). Developmentally regulated postsynaptic localization of a metabotropic glutamate receptor in rat rod bipolar cells. Cell, 77(3), 361-369.

Nucci, C., Gasperi, V., Tartaglione, R., Cerulli, A., Terrinoni, A., Bari, M., ... Maccarrone, M. (2007). Involvement of the endocannabinoid system in retinal damage after high intraocular pressure-induced ischemia in rats. Invest Ophthalmol Vis Sci, 48(7), 2997-3004. doi:10.1167/iovs.06-1355

O'Brien, B.J., Richardson, R.C. \& Berson, D.M. (2003) Inhibitory network properties shaping the light evoked responses of cat alpha retinal ganglion cells. Vis Neurosci 20:351-361

Olah, Z., Szabo, T., Karai, L., Hough, C., Fields, R. D., Caudle, R. M., ... ladarola, M. J. (2001). Ligand-induced dynamic membrane changes and cell deletion conferred by vanilloid receptor 1. J Biol Chem, 276(14), 11021-11030. doi:10.1074/jbc.M008392200

Oleary, C., McNaughten, J. E., Stitt, A. W., McGeown, G. J., \& Curtis, T. M. (2014). TRPV1 channels are functionally expressed in the retinal microvascular endothelium and contribute to retinal angiogenic signalling in vitro. Invest Ophthalmol Vis Sci, 55(13), 5373-5373.

Omori, Y., Araki, F., Chaya, T., Kajimura, N., Irie, S., Terada, K., . . Furukawa, T. (2012). Presynaptic dystroglycan-pikachurin complex regulates the proper synaptic connection between retinal photoreceptor and bipolar cells. J Neurosci, 32(18), 6126-6137. doi:10.1523/jneurosci.0322-12.2012

Orlandi, C., Posokhova, E., Masuho, I., Ray, T. A., Hasan, N., Gregg, R. G., \& Martemyanov, K. A. (2012). GPR158/179 regulate $G$ protein signaling by controlling localization and activity of the RGS7 complexes. $J$ Cell Biol, 197(6), 711-719. doi:10.1083/jcb.201202123

Osterhout, J. A., Josten, N., Yamada, J., Pan, F., Wu, S. W., Nguyen, P. L., . . . Huberman, A. D. (2011). Cadherin-6 mediates axon-target matching in a non-image-forming visual circuit. Neuron, 71(4), 632-639.

doi:10.1016/j.neuron.2011.07.006

Panda, S., Antoch, M. P., Miller, B. H., Su, A. I., Schook, A. B., Straume, M., . . . Hogenesch, J. B. (2002). Coordinated transcription of key pathways in the mouse by the circadian clock. Cell, 109(3), 307-320.

Pardue, M. T., McCall, M. A., LaVail, M. M., Gregg, R. G., \& Peachey, N. S. (1998). A naturally occurring mouse model of X-linked congenital stationary night blindness. Invest Ophthalmol Vis Sci, 39(12), 2443-2449.

Patapoutian, A., Tate, S. \& Woolf, C.J. (2009) Transient receptor potential channels: targeting pain at the source. Nature Reviews Drug Discovery 8:55-68. 
Peachey, N.S., Ray, T.A., Florijn, R., Rowe, L.B., Sjoerdsma, T., ContrerasAlcantara, S., Baba, K., Tosini, G., Pozdeyev, N., luvone, P.M., et al., (2012). GPR179 is required for depolarizing bipolar cell function and is mutated in autosomal-recessive complete congenital stationary night blindness. Am J Hum Genet 90(2):331-339.

Peachey, N. S., Ridder, W. H., 3rd, \& Seeliger, M. W. (2007). Electrophysiologic assessment of the mouse visual system. Doc Ophthalmol, 115(3), 125. doi:10.1007/s10633-007-9080-y

Pearring, J. N., Bojang, P., Jr., Shen, Y., Koike, C., Furukawa, T., Nawy, S., \& Gregg, R. G. (2011). A role for nyctalopin, a small leucine-rich repeat protein, in localizing the TRP melastatin 1 channel to retinal depolarizing bipolar cell dendrites. J Neurosci, 31(27), 10060-10066. doi:10.1523/jneurosci.1014-11.2011

Pearson, J. T., \& Kerschensteiner, D. (2015). Ambient illumination switches contrast preference of specific retinal processing streams. J Neurophysiol, 114(1), 540-550.

Peréz De Sevilla Muller, L., Shelley, J., and Weiler, R. (2007). Displaced amacrine cells of the mouse retina. J Comp Neurol 505, 177-189.

Peters, A., \& Feldman, M. L. (1976). The projection of the lateral geniculate nucleus to area 17 of the rat cerebral cortex. I. General description. $J$ Neurocytol, 5(1), 63-84.

Peters, A., \& Payne, B. R. (1993). Numerical relationships between geniculocortical afferents and pyramidal cell modules in cat primary visual cortex. Cereb Cortex, 3(1), 69-78.

Petrusca, D., Grivich, M. I., Sher, A., Field, G. D., Gauthier, J. L., Greschner, M., ... Litke, A. M. (2007). Identification and characterization of a Y-like primate retinal ganglion cell type. J Neurosci, 27(41), 11019-11027. doi:10.1523/jneurosci.2836-07.2007

Pinto, L. H., Vitaterna, M. H., Shimomura, K., Siepka, S. M., Balannik, V., McDearmon, E. L., . . . Takahashi, J. S. (2007). Generation, identification and functional characterization of the nob4 mutation of Grm6 in the mouse. Vis Neurosci, 24(1), 111-123. doi:10.1017/s0952523807070149

Planells-Cases, R., Garcia-Sanz, N., Morenilla-Palao, C., \& Ferrer-Montiel, A. (2005). Functional aspects and mechanisms of TRPV1 involvement in neurogenic inflammation that leads to thermal hyperalgesia. Pflugers Arch, 451(1), 151-159. doi:10.1007/s00424-005-1423-5

Por, E.D., Bierbower, S.M., Berg, K.A., Gomez, R., Akopian, A.N., Wetsel, W.C. \& Jeske, N.A. (2012) $\beta$-arrestin-2 desensitizes the transient receptor potential vanilloid 1 (TRPV1) channel. American Society for Biochem \& and Molecular Biology, Inc. 287(44):37552-63.

Porcella, A., Maxia, C., Gessa, G. L., \& Pani, L. (2000). The human eye expresses high levels of $\mathrm{CB} 1$ cannabinoid receptor mRNA and protein. Eur J Neurosci, 12(3), 1123-1127.

Pourcho, R. G., \& Goebel, D. J. (1983). Neuronal subpopulations in cat retina which accumulate the GABA agonist, $(3 \mathrm{H})$ muscimol: A combined Golgi 
and autoradiographic study. J Comp Neurol, 219(1), 25-35.

doi:10.1002/cne.902190104

Protti, D.A., Gerschenfeld, H.M., and Llano, I. (1997). GABAergic and glycinergic IPSCs in ganglion cells of rat retinal slices. J Neurosci 17, 6075-6085.

Protti, D.A. \& Llano, I (1998) Calcium currents and calcium signaling in rod bipolar cells of rat retinal slices. $J$ Neurosci 18:3715-24.

Protti, D.A., Di Marco, S., Huang, J.Y., Vonhoff, C.R., Nguyen, V., and Solomon, S.G. (2014). Inner retinal inhibition shapes the receptive field of retinal ganglion cells in primate. J Physiol 592, 49-65.

Provencio, I., Cooper, H. M., \& Foster, R. G. (1998). Retinal projections in mice with inherited retinal degeneration: implications for circadian photoentrainment. J Comp Neurol, 395(4), 417-439.

Provencio, I., \& Foster, R. G. (1995). Circadian rhythms in mice can be regulated by photoreceptors with cone-like characteristics. Brain Res, 694(1-2), 183-190.

Purves D, Augustine GJ, Fitzpatrick D, et al., (2001) Neuroscience. 2nd edition. Sunderland (MA): Sinauer Associates

Ray, T. A., Heath, K. M., Hasan, N., Noel, J. M., Samuels, I. S., Martemyanov, K. A., ... Gregg, R. G. (2014). GPR179 is required for high sensitivity of the mGluR6 signaling cascade in depolarizing bipolar cells. J Neurosci, 34(18), 6334-6343. doi:10.1523/jneurosci.4044-13.2014

Renteria, R., Jeanes, Z. M., \& Morrisett, R. A. (2014). Ethanol Attenuation of Long-Term Depression in the Nucleus Accumbens Can Be Overcome by Activation of TRPV1 Receptors. Alcoholism: Clinical and Experimental Research, 38(11), 2763-2769. doi:10.1111/acer.12542

Renteria, R. C., Tian, N., Cang, J., Nakanishi, S., Stryker, M. P., \& Copenhagen, D. R. (2006). Intrinsic ON responses of the retinal OFF pathway are suppressed by the ON pathway. J Neurosci, 26(46), 11857-11869. doi:10.1523/jneurosci.1718-06.2006

Richardson, J. D., and Vasko, M. R. (2002). Cellular mechanisms of neurogenic inflammation. J. Pharmacol. Exp. Ther. 302, 839-845

Ritter, S., \& Dinh, T. T. (1988). Capsaicin-induced neuronal degeneration: silver impregnation of cell bodies, axons, and terminals in the central nervous system of the adult rat. J Comp Neurol, 271(1), 79-90. doi:10.1002/cne.902710109

Ritter, S., \& Dinh, T. T. (1990). Capsaicin-induced neuronal degeneration in the brain and retina of preweanling rats. J Comp Neurol, 296(3), 447-461. doi:10.1002/cne.902960310

Ritter, S., \& Dinh, T. T. (1992). Age-related changes in capsaicin-induced degeneration in rat brain. J Comp Neurol, 318(1), 103-116. doi:10.1002/cne.903180108

Rodieck, R.W. \& Stone, J (1965) Analysis of receptive fields of cat retinal ganglion cells. J Neurophysiol 28(5): 833-849

Roska, B., Molnar, A. \& Werblin, F.S. (2006) Parallel processing in retinal ganglion cells: how integration of space-time patterns of excitation and inhibition form the spiking output. J Neurophysiol 95:3810-3822 
Roska, B., Nemeth, E., Orzo, L. \& Werblin, F.S. (2000) Three levels of lateral inhibition: a space-time study of the retina of the tiger salamander. $J$. Neurosci 20:1941-1951

Roska, B., Nemeth, E., \& Werblin, F. S. (1998). Response to change is facilitated by a three-neuron disinhibitory pathway in the tiger salamander retina. $J$ Neurosci, 18(9), 3451-3459.

Roska, B., \& Werblin, F. (2001). Vertical interactions across ten parallel, stacked representations in the mammalian retina. Nature, 410(6828), 583-587. doi: $10.1038 / 35069068$

Ross RA (2003). Anandamide and vanilloid TRPV1 receptors. Br J Pharmacol 140: 790-801.

Russell, T.L., and Werblin, F.S. (2010). Retinal synaptic pathways underlying the response of the rabbit local edge detector. JNeurophysiol 103, 27572769.

Ryskamp, D. A., Redmon, S., Jo, A. O., \& Krizaj, D. (2014). TRPV1 and Endocannabinoids: Emerging Molecular Signals that Modulate Mammalian Vision. Cells, 3(3), 914-938. doi:10.3390/cells3030914

Ryskamp, D.A., Witkovsky, P., Barabas, P., Huang, W., Koehler, C., Akimov, N.P. et al. (2011) The polymodal ion channel transient receptor potential vanilloid 4 modulates calcium flux, spiking rate, and apoptosis of mouse retinal ganglion cells. J Neurosci 31(19):7089-101.

Sagdullaev, B.T., DeMarco, P.J., and McCall, M.A. (2004). Improved contact lens electrode for corneal ERG recordings in mice. DocOphthalmol 108, 181184.

Sagdullaev, B.T., and McCall, M.A. (2005). Stimulus size and intensity alter fundamental receptive-field properties of mouse retinal ganglion cells in vivo. Vis Neurosci 22, 649-659

Sagdullaev, B. T., McCall, M. A., \& Lukasiewicz, P. D. (2006). Presynaptic inhibition modulates spillover, creating distinct dynamic response ranges of sensory output. Neuron, 50(6), 923-935. doi:10.1016/j.neuron.2006.05.015

Sakamoto, K., Kuroki, T., Okuno, Y., Sekiya, H., Watanabe, A., Sagawa, T., . . . Ishii, K. (2014). Activation of the TRPV1 channel attenuates N-methyl-Daspartic acid-induced neuronal injury in the rat retina. Eur $J$ Pharmacol, 733, 13-22.

Sampath, A.P., Matthews, H.R., Cornwall, M.C., Bandarchi, J. \& Fain, G.L. (1999). Light-dependent changes in outer segment free- $\mathrm{Ca}^{2+}$ concentration in salamander cone photoreceptors. J. Gen. Physiol. 113: 267-277

Sampath, A.P., Rieke, F. (2004) Selective transmission of single photon responses by saturation at the rod-to-rod bipolar synapse. Neuron. 41: $431-443$

Sanchez, J. F., Krause, J. E., \& Cortright, D. N. (2001). The distribution and regulation of vanilloid receptor VR1 and VR1 5' splice variant RNA expression in rat. Neuroscience, 107(3), 373-381. 
Sanes, J. R., \& Masland, R. H. (2015). The types of retinal ganglion cells: current status and implications for neuronal classification. Annu Rev Neurosci, 38, 221-246. doi:10.1146/annurev-neuro-071714-034120

Sappington, R. M., Sidorova, T., Ward, N. J., Chakravarthy, R., Ho, K. W., \& Calkins, D. J. (2015). Activation of transient receptor potential vanilloid-1 (TRPV1) influences how retinal ganglion cell neurons respond to pressure-related stress. Channels (Austin), 9(2), 102-113

Sappington, R.M., Stdorova, T., Long, D.J., \& Calkins, D.J. (2009). TRPV1: Contribution to retinal ganglion cell apoptosis and increased intracellular $\mathrm{Ca} 2+$ with exposure to hydrostatic pressure. Invest. Ophthal. \& Visual Science. 50 (2): 717-728

Scalabrino, M. L., Boye, S. L., Fransen, K. M., Noel, J. M., Dyka, F. M., Min, S. H., . . Boye, S. E. (2015). Intravitreal delivery of a novel AAV vector targets ON bipolar cells and restores visual function in a mouse model of complete congenital stationary night blindness. Hum Mol Genet, 24(21), 6229-6239.

Schmidt, T. M., Chen, S. K., \& Hattar, S. (2011). Intrinsically photosensitive retinal ganglion cells: many subtypes, diverse functions. Trends Neurosci, 34(11), 572-580. doi:10.1016/j.tins.2011.07.001

Schneeweis, D.M. \& Schnapf, J.L. (1995) Photovoltage of rods and cones in the macaque retina. Science 268:1053-1056

Schubert, T., Kerschensteiner, D., Eggers, E. D., Misgeld, T., Kerschensteiner, M., Lichtman, J. W., . . Wong, R. O. (2008). Development of presynaptic inhibition onto retinal bipolar cell axon terminals is subclass-specific. $J$ Neurophysiol, 100(1), 304-316. doi:10.1152/jn.90202.2008

Sekaran, S., Lall, G.S., Ralphs, K.L., Wolstenholme, A.J., Lucas, R.J., Foster, R.G. \& Hankins, M.W. (2007) 2-Aminoethoxydiphenylborane is an acute inhibitor of direct photosensitive retinal ganglion cell activity in vitro and in vivo. J Neurosci. 27(15):3981-3986

Sekirnjak, C., Hottowy, P., Sher, A., Dabrowski, W., Litke, A. M., \& Chichilnisky, E. J. (2006). Electrical stimulation of mammalian retinal ganglion cells with multielectrode arrays. J Neurophysiol, 95(6), 3311-3327.

Shen, Y. Heimel, J.A. Kamermans, M., Peachey, N.S., Gregg, R.G. \& Nawy, S. (2009). A transient receptor potential-like channel mediates synaptic transmission in rod bipolar cells. J. of Neuroscience. 29(19): 6088-6093

Shim, H. Wang, C.T., Chen, Y.L., Chau, V.Q., Fu, K.G., Yang, J., McQuiston, A.R., Fisher, R.A., Chen, C.K. (2012). Defective retinal depolarizing bipolar cells (DBCs) in regulators of G-protein signaling (RGS) 7 and 11 double null mice. J. Biol Chem. 287: 14873-14879

Slaughter, M.M. \& Miller, R.F. (1985) Characterization of an extended glutamate receptor on the ON bipolar neuron in the vertebrate retina. J. Neurosci. 5: 224-233

Smith, E. S., Fabian, P., Rosenthal, A., Kaddour-Djebbar, A., \& Lee, H. J. (2015). The roles of central amygdala D1 and D2 receptors on attentional performance in a five-choice task. Behav Neurosci, 129(5), 564-575. doi: $10.1037 / \mathrm{bne} 0000077$ 
Snellman, J., Kaur, T., Shen, Y., Nawy, S. (2008). Regulation of ON bipolar cell activity. Prog Retin Eye Res. 27: 450-463

Snellman, J., Zenisek, D., \& Nawy, S. (2009). Switching between transient and sustained signalling at the rod bipolar-All amacrine cell synapse of the mouse retina. J Physiol, 587(Pt 11), 2443-2455. doi:10.1113/jphysiol.2008.165241

Strettoi, E. Dacheux, R.F.\& Raviola, E. (1990) Synaptic connection of rod bipolar cells in the inner plexiform layer of the rabbit retina. J Comp Neurol 295:449-466

Strettoi, E., Raviola, E. \& Dacheux, R.F (1992) Synaptic connection of the narrow-field, bistratified rod amacrine cell (All) in the inner retina of the rabbit. J Comp Neurol 325:152-168

Stryer, L. (1991) Visual excitation and recovery. J Biol Chem 266:10711-24

Su, J., Haner, C. V., Imbery, T. E., Brooks, J. M., Morhardt, D. R., Gorse, K., . . . Fox, M. A. (2011). Reelin is required for class-specific retinogeniculate targeting. J Neurosci, 31(2), 575-586.

Sugimoto, K., Kissin, I. \& Strichartz, G. (2008). A high concentration of resiniferatoxin inhibits ion channel function in clonal neuroendocrine cells. International Anesthesia Research Society 107: 318-324

Sun, Wenzhi, Li, Ning, \& He, Shigang. (2002). Large-scale morphological survey of mouse retinal ganglion cells. The Journal of Comparative Neurology 451: 115-126

Szallasi A, Blumberg PM. 1999.Vanilloid (capsaicin) receptors and mechanisms. Pharmacol Rev. 51:159-212

Tian, N. \& Copenhagen, D.R. (2003) Visual stimulation is required for refinement of ON and OFF pathways in postnatal retina. Neuron 39: 85-96

Tikidji-Hamburyan, A., Reinhard, K., Seitter, H., Hovhannisyan, A., Procyk, C. A., Allen, A. E., ... Munch, T. A. (2015). Retinal output changes qualitatively with every change in ambient illuminance. Nat Neurosci, 18(1), 66-74.

Umino, Y., Solessio, E., \& Barlow, R. B. (2008). Speed, spatial, and temporal tuning of rod and cone vision in mouse. J Neurosci, 28(1), 189-198.

van der Stelt \& Di Marzo (2004) Endovanilloids: Putative endogenous ligands of transient receptor potential vanilloid 1 channels. Eur. J. Biochem.271:1827-1834.

van Genderen, M.M., Bijveld, M.M., Claassen, Y.B., Florijn, R.J., Pearring, J.N., Meire, F.M., McCall, M.A., Riemslag, F.C., Gregg, R.G., Bergen, A.A., et al. (2009). Mutations in TRPM1 are a common cause of complete congenital stationary night blindness. Am J Hum Genet 85, 730-736.

van Wyk, M., Wässle, H., and Taylor, W.R. (2009). Receptive field properties of ON- and OFF-ganglion cells in the mouse retina. Vis Neurosci 26, 297308

Vardi N, Matesic DF, Manning DR, Liebman PA \& Sterling P (1993). Identification of a G-protein in depolarizing rod bipolar cells. Vis Neurosci 10, 473-478

Vardi N, Duvoisin R, Wu G \& Sterling P (2000). Localization of mGluR6 to dendrites of ON bipolar cells in primate retina. J Comp Neurol 423, 402412 
Venkatachalam, K., \& Montell, C. (2007). TRP channels. Annu Rev Biochem, 76, 387-417. doi:10.1146/annurev.biochem.75.103004.142819

Versaux-Botteri, C., Nguyen-Legros, J., Vigny, A. \& Raoux, N. (1984) Morphology, density and distribution of tyrosine hydroxylase-like immunoreactive cells in the retina of mice. Brain Research 301(1): 192197.

Vielma, A. H., Agurto, A., Valdes, J., Palacios, A. G., \& Schmachtenberg, O. (2014). Nitric oxide modulates the temporal properties of the glutamate response in type 4 OFF bipolar cells. PLoS One, 9(12), e114330. doi:10.1371/journal.pone.0114330

Völgyi, B., Chheda, S., and Bloomfield, S.A. (2009). Tracer coupling patterns of the ganglion cell subtypes in the mouse retina. J Comp Neurol 512, 664687.

Völgyi, B., Deans, M.R., Paul, D.L., and Bloomfield, S.A. (2004). Convergence and segregation of the multiple rod pathways in mammalian retina. $J$ Neurosci 24, 11182-11192.

Walch, O. J., Zhang, L. S., Reifler, A. N., Dolikian, M. E., Forger, D. B., \& Wong, K. Y. (2015). Characterizing and modeling the intrinsic light response of rat ganglion-cell photoreceptors. J Neurophysiol, 114(5), 2955-2966.

Ward, N. J., Ho, K. W., Lambert, W. S., Weitlauf, C., \& Calkins, D. J. (2014). Absence of transient receptor potential vanilloid-1 accelerates stressinduced axonopathy in the optic projection. J Neurosci, 34(9), 3161-3170. doi: 10.1523/JNEUROSCI.4089-13.2014

Wässle $H$ (2004). Parallel processing in the mammalian retina. Nat Rev Neurosci $5,747-757$

Wässle, H., Puller, C., Muller, F., \& Haverkamp, S. (2009). Cone contacts, mosaics, and territories of bipolar cells in the mouse retina. J Neurosci, 29(1), 106-117.

Weitlauf, C., Ward, N. J., Lambert, W. S., Sidorova, T. N., Ho, K. W., Sappington, R. M., \& Calkins, D. J. (2014). Short-term increases in transient receptor potential vanilloid-1 mediate stress-induced enhancement of neuronal excitation. J Neurosci, 34(46), 15369-15381.

Wellis, D. P., \& Werblin, F. S. (1995). Dopamine modulates GABA $_{c}$ receptors mediating inhibition of calcium entry into and transmitter release from bipolar cell terminals in tiger salamander retina. J Neurosci, 15(7 Pt 1), 4748-4761.

Werblin, F. (1991). Synaptic connections, receptive fields, and patterns of activity in the tiger salamander retina. A simulation of patterns of activity formed at each cellular level from photoreceptors to ganglion cells [the Friendenwald lecture]. Investigative ophthalmology \& visual science 32, 459-483.

Werblin, F.S. (2010). Six different roles for crossover inhibition in the retina: correcting the nonlinearities of synaptic transmission. VisNeurosci 27, 18. 
Werblin, F.S. and J.E. Dowling, Organization of the retina of the mudpuppy, Necturus maculosus. II. Intracellular recording. J Neurophysiol, 1969. 32(3): p. 339-55

Werblin, F., Roska, B. \& Balya, D. (2001). Parallel processing in the mammalian retina: lateral and vertical interactions across stacked representations. Progress in Brain Research 131:229-238.

Wexler, E. M., Stanton, P. K., \& Nawy, S. (1998). Nitric oxide depresses GABA $A$ receptor function via coactivation of cGMP-dependent kinase and phosphodiesterase. J Neurosci, 18(7), 2342-2349.

White, J.P.M., Urban, L. \& Nagy, I. (2011). TRPV1 function in health and disease. Current Pharm. Biotech. 12: 130-144.

Williams, R. W., \& Goldowitz, D. (1992). Structure of clonal and polyclonal cell arrays in chimeric mouse retina. Proc Natl Acad Sci U S A, 89(4), 11841188.

Witkovsky, P. (2004). Dopamine and retinal function. Doc Ophthalmol, 108(1), 17-40.

Yau, K-W (1994) Phototransduction mechanisms in retinal rods and cones. Invest Ophthal vis Sci. 35:9-32.

Yazulla, S. (2008). Endocannabinoids in the retina: from marijuana to neuroprotection. Prog Retin Eye Res, 27(5), 501-526.

doi:10.1016/j.preteyeres.2008.07.002

Yazulla, S., Studholme, K.M., McIntosh, H.H. \& Fan, S.F. (2000). Cannabinoid receptors on goldfish retinal bipolar cells: Electron microscope immunocytochemistry and whole-cell recordings. Visual Neuroscience 17, 391-401.

Yee, C.W., Toychiev, A.H. \& Sagdullaev, B.T. (2012) Network deficiency exacerbates impairment in a mouse model of retinal degeneration. Frontiers in Systems Neurosc. 6(8): 1-13.

Zabouri, N., Ptito, M., Casanova, C., \& Bouchard, J. F. (2011). Fatty acid amide hydrolase expression during retinal postnatal development in rats. Neuroscience, 195, 145-165. doi:10.1016/j.neuroscience.2011.08.008

Zacharova, G., \& Palecek, J. (2009). Parvalbumin and TRPV1 receptor expression in dorsal root ganglion neurons after acute peripheral inflammation. Physiol Res, 58(2), 305-309.

Zaghloul, K.A., Boahen, K., and Demb, J.B. (2003). Different circuits for ON and OFF retinal ganglion cells cause different contrast sensitivities. $J$ Neurosci 23, 2645-2654.

Zeitz, C., Jacobson, S. G., Hamel, C. P., Bujakowska, K., Neuille, M., Orhan, E., . .. Audo, I. (2013). Whole-exome sequencing identifies LRIT3 mutations as a cause of autosomal-recessive complete congenital stationary night blindness. Am J Hum Genet, 92(1), 67-75. doi:10.1016/j.ajhg.2012.10.023

Zeitz, C., Robson, A. G., \& Audo, I. (2015). Congenital stationary night blindness: an analysis and update of genotype-phenotype correlations and pathogenic mechanisms. Prog Retin Eye Res, 45, 58-110. doi:10.1016/j.preteyeres.2014.09.001 
Zhang, C \& McCall, M.A. (2012) Receptor targets of amacrine cells. Vis Neurosci 29:11-29.

Zhang, C., Rompani, S.B., Roska, B., and McCall, M.A. (2014). Adenoassociated virus-RNAi of GlyRalpha1 and characterization of its synapse-specific inhibition in OFF alpha transient retinal ganglion cells. $J$ Neurophysiol 112, 3125-3137.

Zhang, D. Q., Zhou, T., Ruan, G. X., \& McMahon, D. G. (2005). Circadian rhythm of Period1 clock gene expression in NOS amacrine cells of the mouse retina. Brain Res, 1050(1-2), 101-109. doi:10.1016/j.brainres.2005.05.042

Zhang, J., Jung, C.S., and Slaughter, M.M. (1997). Serial inhibitory synapses in retina. Vis Neurosci 14, 553-563

Zhang, Y., Kim, I. J., Sanes, J. R., \& Meister, M. (2012). The most numerous ganglion cell type of the mouse retina is a selective feature detector. Proc Natl Acad Sci U S A, 109(36), E2391-2398. doi:10.1073/pnas.1211547109

Zhu, Y., Xu, J., Hauswirth, W.W., and DeVries, S.H. (2014). Genetically targeted binary labeling of retinal neurons. J Neurosci 34, 7845-7861.

Zimov, S. \& Yazulla, S. (2007). Vanilloid receptor 1 (TRPV1/VR1) co-localizes with fatty acid amide hydrolase (FAAH) in retinal amacrine cells. Visual Neuroscience 24: 581-591 


\section{APPENDIX}

\section{STATISTICAL ANALYSIS}

The multi-electrode array (MEA) is a useful tool for collecting large numbers of cells from single retinas simultaneously (Marre et al., 2012; Petrusca et al., 2007). Previous publications have assessed correlations between different cells (Elstrott et al., 2008; Farajian, Pan, Akopian, Volgyi, \& Bloomfield, 2011; Hamby, Rosa, Hsu, \& Feller, 2015), categorize many functional types (Anishchenko et al., 2010; Li et al., 2015; Marre et al., 2012; Walch et al., 2015), assess responses of these types to a large variety of stimuli (Hamby et al., 2015; Pearson \& Kerschensteiner, 2015; Tikidji-Hamburyan et al., 2015) and analyze the affects of disease (Chen et al., 2015; Della Santina, Inman, Lupien, Horner, \& Wong, 2013; Fransen, Pangeni, Pyle, \& McCall, 2015; Gonzalez-Menendez, Reinhard, Tolivia, Wissinger, \& Munch, 2015; Sekirnjak et al., 2006). With the MEA caution is required when making statistical comparisons between groups as I have. The t-statistic, which is the most used between two samples to determine significance, is dependent on the variability in the samples (Student, 1908)(Krzywinski \& Altman, 2014a). The example t-test equation below is used to compare two independent samples with normal distribution, equal variance and unequal number. 


$$
t=\frac{\bar{X}_{1}-\bar{X}_{2}}{s_{X_{1} X_{2}} \cdot \sqrt{\frac{1}{n_{1}}+\frac{1}{n_{2}}}}
$$

$\bar{X}_{1}-\bar{X}_{2}=$ difference in the sample means

sx $=$ Standard deviation of the sample

$s^{2} x=$ Variance of the sample

$\mathrm{n}=$ number of the sample

$$
s_{X_{1} X_{2}}=\sqrt{s_{X_{1}}^{2}+s_{X_{2}}^{2}}
$$

The t-statistic is determined by taking the differences of the two population means and dividing it by the sum of the variability in the two populations and the sum of the inverse of the number. Therefore as the variability in the samples increases, the t-statistic will decrease, even if the means don't change. Also, if the numbers in the samples increase, the t-statistic will also increase. As the tstatistic increases, the chance of finding a significant difference between the means also increase ( $p$ decreases).

With the MEA there are multiple sources of variability in the system. Variability is innate in both the technical collection of the data and the biological differences in cells, retinas, and mice. To account for the variability, repetitive samples are taken during the recording process. I present 10 repetitions of each luminance to measure the variability in the spiking responses. I compare the average response within cell classes to measure the variability in each class. I can compare the average class response in each retina or mouse to measure the variability in the animal. When experiments involve multiple distributions and therefore multiple sources of variability, the total distribution is the sum of the 
individual distributions and the variability is the sum of the variabilities or variances (Blainey, Krzywinski, \& Altman, 2014).

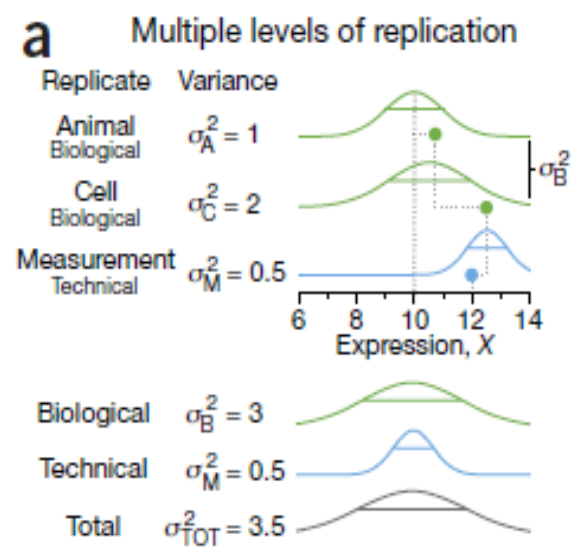

Figure 1. Experiments with multiple replications have multiple variances $\left(\sigma^{2}\right)$ from both technical and biological sources. The total variance in the sample is the sum of the individual variances (from Blainey et al., 2014)

With the MEA, large numbers of cells are collected from small numbers of

mice. Since the chances of finding a significant difference between groups

increases as numbers increase, then making comparisons between large populations of cells increases the probability of finding a statistical difference. However, with the decrease in the numbers of mice sampled, the variability between mice is most likely under sampled and under estimated in the total variance. As mentioned before, as the variance decreases, the t-statistic increases, again increasing the probability of finding a significant difference. Therefore, the increase in number and decrease in variance must be adjusted for to prevent type I errors (finding a significant difference that does not actually exist; (Blainey et al., 2014).

Ultimately using the mouse as the $\mathrm{n}$ in statistical comparisons is usually the best solution, however a balance must be made between what is optimal for each technique. Here, the power in the MEA is the ability to sample from many 
numbers and classes of cells simultaneously. Experiments should be designed to give the best chance of determining real differences only with minimal waste (especially when involving animals). In order to obtain suitable power (> 80\%) given the variability and effect size between my samples, tens to hundreds of units should be compared (G-Power analysis). Decreasing the power decreases the probability of finding a difference that actually exists (type II error; (Krzywinski \& Altman, 2014b). Regarding my MEA data, using the mouse as the $\mathrm{n}$ for all comparisons is not feasible for those numbers given the resources and time needed for data collection and analysis. In the analysis of my experiments I used retinal pieces as the unit when feasible, such as in the comparisons of proportions of cell types and the transience of the responses. I compared my results obtained from retinal pieces to those obtained using the cell as the unit and obtained the same statistical results. For the comparisons of light responses within the cell classes, I used the cell as the $n$ and considered $p$ values $\leq 0.01$ to be significant to decrease the chance of committing a type 1 error. 


\section{CURRICULUM VITAE}

Jennifer M. Noel

1213 S. $1^{\text {st }}$ St.

Louisville, KY 40203

United States

(502) 298-9360

inbrod01@gmail.com

\section{Education}

University of Louisville, Louisville, KY

Ph.D. Candidate: Anatomical Sciences and Neurobiology

Project: I investigated the roles of Trp receptor/channels in vision

M.S. Anatomical Sciences and Neurobiology

Thesis: "Transient receptor potential cation channel

2013 vanilloid 1 (TRPV1) modulates retinal ganglion cell responses to light"

M.S. Candidate: Interdisciplinary Studies Areas of Concentration: Neuroscience

Thesis: "lodoacetic acid creates an inducible model of retinal

2008-2010 degeneration in swine"

B.S. in Psychology

Areas of Concentration: Pre-medical path

Honors: Graduated Cum Laude

\section{Awards}

Grant in Aid of Research, Sigma Xi Scientific Society

Research Fellowship, University of Louisville Medical

School

Travel Grant, National Eye Institute to the Association for Research in Vision and Ophthalmology (ARVO) Annual Meeting 
Honors Research Program, University of Louisville,

2006-2007 Department of Psychological and Brain Sciences

Academic and Fine Arts Scholarships, Milligan College, TN

\section{Research Experience}

University of Louisville, Louisville, KY

Research Fellow and Ph.D. Candidate -

2010 - present

Department of Anatomical Sciences and Neurobiology

Mentor: Dr. Maureen McCall

Research: Characterization of the role of Trp channel/receptors in the retina. I specifically assessed the function and expression of TRPV1 in the retina and determined that TRPV1 modulates signal transduction to likely enhance the perception of details in a visual scene and dim light in the dark. I recorded responses of retinal ganglion cells, the output cells that communicate with the brain. I compared light responses of retinal ganglion cells between mice with or without TRPV1 receptors. I simultaneously used immunohistochemistry to determine the specific retinal cells that express TRPV1.

I similarly assessed TRPM1 protein cascade function in the retina. I compared electroretinograms from mice with or without the cascade protein LRIT3 and determined that LRIT3 is necessary for TRPM1 expression. I used immunohistochemistry to help discover the vital role of LRIT3 in proper neurotransmission from photoreceptors to their bipolar cell partners.

Master's Candidate - Department of Interdisciplinary Studies $\quad 2008$ - 2010 Mentor: Dr. Paul DeMarco

Research: The creation and characterization of a convenient and inducible swine model of retinal degeneration. Swine provide a large animal model of eye disease that is the most similar to human among non-primates.

lodoacetic acid (IAA) administered intravenously leads to a selective loss of photoreceptors, creating a rapidly inducible model of the human disease Retinitis Pigmentosa. We characterized the dose dependent level of retinal damage using full-field and multifocal electroretinograms, clinical exams, and histology. We then used the model to investigate treatments for retinal degeneration.

Research Technician - Department of Ophthalmology $2007-2010$ Supervisor: Dr. Henry J. Kaplan

Research

Project 1: We examined the effects of Granulocyte Colony Stimulating Factor (G-CSF) on mice with toxin (sodium iodate) induced or genetically based retinal degeneration. The effects of G-CSF treatment on the retina was assessed through electroretinograms, behavioral tests (optokinetic 
response) and histology. We discovered that treatment with G-CSF preserved and/or restored visual function in mice.

Project 2: The creation and characterization of a mini-swine model of the human retinal degenerative disease, Retinitis Pigmentosa. Due to their limited size (<200 lbs.), mini-swine are a more useful large animal model than adult domestic swine ( $>400 \mathrm{lbs}$.). The $\mathrm{P} 23 \mathrm{H}$ mutation in the rhodopsin gene is the most common cause of human Retinitis Pigmentosa. We characterized the time course and severity of disease progression in several lines of the $\mathrm{P} 23 \mathrm{H}$ mini-swine. We used full-field and multifocal

electroretinograms, clinical exams, and histology to characterize the model. We then used mini-swine and mini/domestic swine hybrids to test treatments for retinal degeneration.

Honors Undergraduate Research Program - Department $\quad 2006$ - 2007 of Psychology and Brain Sciences

Mentor: Dr. Maureen McCall

Research: I investigated the role of the inhibitory neurotransmitter receptor, GABAc, in the retina. Specifically, I determined that retinal ganglion cells have normal morphology in the absence of GABA receptors. I used confocal microscopy to collect 3D images of fluorescently labeled retinal ganglion cells in whole mouse retinas. I then analyzed the structural characteristics of the cells and compared them between mice with or without $\mathrm{GABA}$ r receptors.

\section{Related Experience}

Kentucky Lions Eye Center Eye Clinic, Louisville, KY

Clinical Electrophysiology Technician

I performed vision tests to assess the retinal function of referred patients. Tests included full-field and multifocal electroretinograms, visually evoked potentials, electroocculograms, dark adaptation testing, and color vision testing.

\section{Johnson City Medical Center, Johnson City, TN}

Certified Nursing Assistant

2003

I provided basic care for patients coming out of intensive care units.

\section{Publications and Posters}

Scalabrino, M.L., Boye, S.F., Fransen, K.M.H., Noel, J.M., Dyka, F.M., Min, S.H., Ruan, Q., De Leeuw, C.N., Simpson, E.M., Gregg, R.G., McCall, M.A., Peachey, N.S. and Boye, S.E. (2015) Intravitreal delivery of a novel AAV vector targets ON bipolar cells and restores visual function in a mouse model of complete congenital stationary night blindness. Human Molecular Genetics 24(21): 6229-39

Ray, T.A., Heath, K.M., Hasan, N., Noel, J.M., Samuels, I.S., Martemyanov, K.A., Peachey, N.S., McCall, M.A., Gregg, R.G. (2014) GPR179 is required 
for high sensitivity of the mGluR6 signaling cascade in depolarizing bipolar cells. J Neuroscience 34(18): 6334-43.

Ross, J.W., Fernandez de Castro, J.P., Zhao, J., Samuel, M., Walters, E., Rios, C., Bray-Ward, P., Jones, B.W., Marc, R.E., Wang, W., Zhou, L., Noel, J.M., McCall, M.A., DeMarco, P.J., Prather, R.S., Kaplan, H.J. (2012) Generation of an Inbred Miniature Pig Model of Retinitis Pigmentosa. Invest Ophthalmol Vis Sci. 53(1): 501-7.

Noel, J.M., Fernandez de Castro, J.P., Demarco, P.J., Franco, L.M., Wang, W., Vukmanic, E.V., Peng, X., Scott, P.A., Sandell, J.H., Kaplan, H.J., McCall, M.A. (2012) lodoacetic acid, but not sodium iodate, creates an inducible swine model of photoreceptor damage. Exp Eye Res. 97(1): 137-47

Wang, W., Noel, J.M., Kaplan, H.J., Dean, D.C. (2011)

Circulating Reactive Oxidant Causes Apoptosis of Retinal Pigment Epithelium and Cone Photoreceptors in the Mouse Central Retina. Ophthalmol Eye Dis. 3: 45-54.

"LRIT3 and OPL Synaptic Structure and Function"

Paper presentation at the ARVO annual meeting, 2015 Invest Ophthalmol Vis Sci. 56: 2611.

"Proper localization and function of TRPM1 depends on LRIT3 expression in rod depolarizing bipolar cells"

Poster presentation at FASEB retinal conference, 2014

"TRPV1 modulates visual responses from the retina to the brain"

Poster presentation and data blitz at FASEB retinal conference, 2012

"Effects of G-CSF in mouse models of retinal degeneration"

Poster presentation at ARVO annual meeting, 2011

Invest Ophthalmol Vis Sci. 52: 5463.

"IAA Creates an Inducible Model of Retinal Degeneration"

Poster presented at the ARVO annual meeting, 2009

Invest Ophthalmol Vis Sci. 50: 3622.

\section{Memberships}

Society for Neuroscience: Louisville Chapter

Sigma Xi Scientific Society

Association for Research in Vision and Ophthalmology

Golden Key International Honors Society 